\title{
Publications of Los Alamos Research 1984
}

\author{
Compiled by \\ Connie J. Sheridan \\ Wilma J. McClary \\ Jeannette A. Rich \\ LaVerne L. Rodriguez
}

\section{DISCLAIMER}

This report was prepared as an account of work sponsored by an agency of the United States Government. Neither the United States Government nor any agency thereof, nor any of their employees, makes any warranty, express or implied, or assumes any legal liability or responsibility for the accuracy, completeness, or usefulness of any information, apparatus, product, or process disclosed, or represents that its use would not infringe privately owned rights. Reference herein to any specific commercial product, process, or service by trade name, trademark, manufacturer, or otherwise does not necessarily constitute or imply its endorsement, recommendation, or favoring by the United States Government or any agency thereof. The views and opinions of authors expressed herein do not necessarily state or reflect those of the United States Government or any agency thereof.

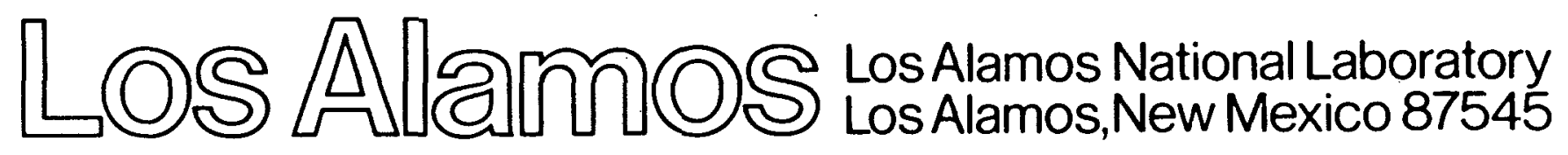

$$
i \text { iv }
$$




\section{DISCLAIMER}

This report was prepared as an account of work sponsored by an agency of the United States Government. Neither the United States Government nor any agency Thereof, nor any of their employees, makes any warranty, express or implied, or assumes any legal liability or responsibility for the accuracy, completeness, or usefulness of any information, apparatus, product, or process disclosed, or represents that its use would not infringe privately owned rights. Reference herein to any specific commercial product, process, or service by trade name, trademark, manufacturer, or otherwise does not necessarily constitute or imply its endorsement, recommendation, or favoring by the United States Government or any agency thereof. The views and opinions of authors expressed herein do not necessarily state or reflect those of the United States Government or any agency thereof. 


\section{DISCLAIMER}

Portions of this document may be illegible in electronic image products. Images are produced from the best available original document. 
4 


\section{CONTENTS}

Introduction $\ldots \ldots \ldots \ldots \ldots \ldots \ldots \ldots \ldots \ldots \ldots \ldots \ldots \ldots \ldots \ldots \ldots \ldots$

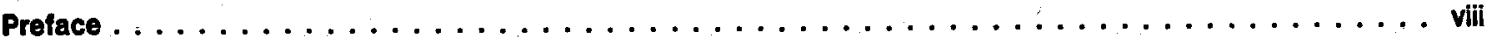

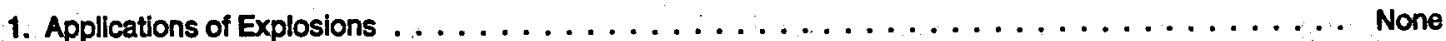

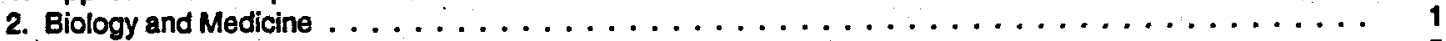

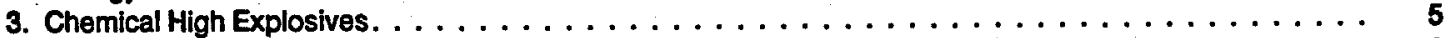

4. Chemical Separations Processes for Plutonium and Uranium $\ldots \ldots \ldots \ldots \ldots \ldots \ldots \ldots \ldots$

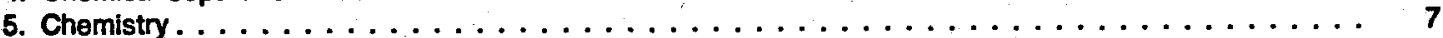

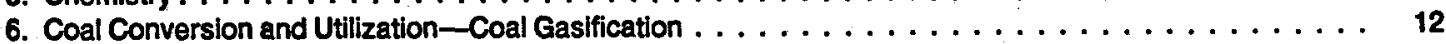

7. Coal Conversion and Utilization-Coal Science and Analysis. . . . . . . . . . . . . . . None

8. Coal Conversion and Utilization-Environmental Protection,

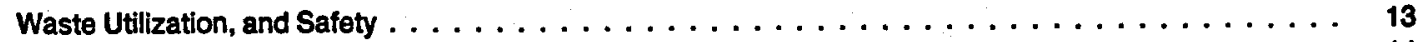

9. Criticality Studies. . . . . . . . . . . . . . . . . . . . . . . . . . . . . . . . 14

10. Electric Energy Systems-Systems Development and Control . . . . . . . . . . . . . . . . . . . . . . 15

11. Energy Conservation-Systems Modeling and Performance Assessment . . . . . . . . . . . . . 16

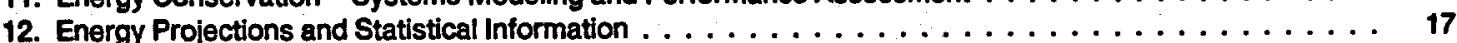

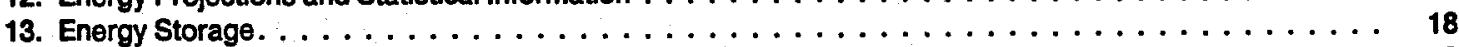

14. Engineering and Equipment. . . . . . . . . . . . . . . . . . . . . . . 19

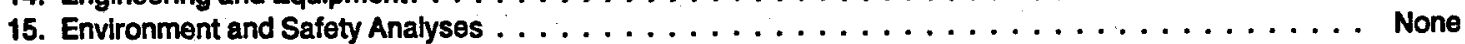

16. Environment Control Technology and Earth Sciences . . . . . . . . . . . . . . . . . 23

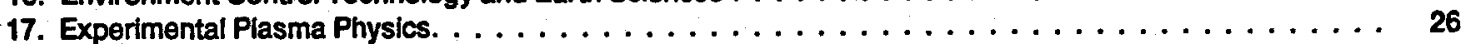

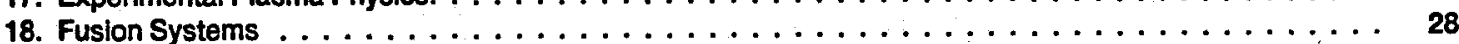

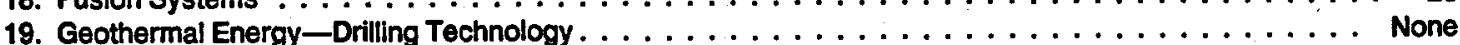

20. Geothermal Energy-Environmental Research. . . . . . . . . . . . . . . . . . . . . . . 29

21. Geothermal Energy_Exploration Technology . . . . . . . . . . . . . . . . . . . . . 30

22. Geothermal Energy_Resource Development . . . . . . . . . . . . . . . . . . . . 31

23. Gas Cooled Reactor Technology . . . . . . . . . . . . . . . . . . . . . . . . . None

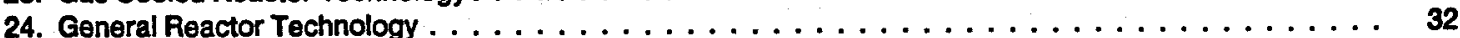

25. General, Miscellaneous, and Progress Reports (Nonnuclear). . . . . . . . . . . . . . . . . . 33

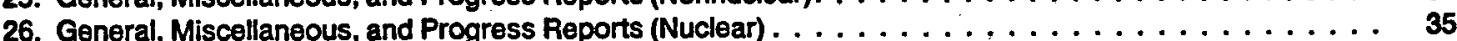

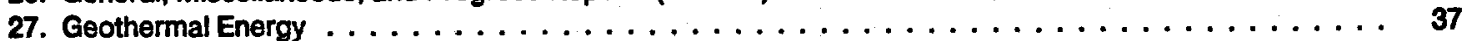

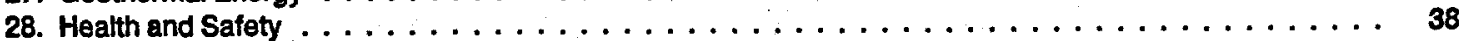

29. Heating and Cooling-Residental and Commercial Applications . . . . . . . . . . . . . . . . . . . 40

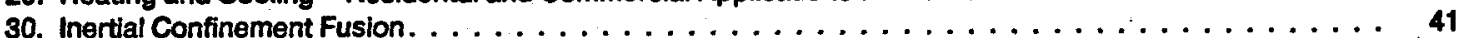

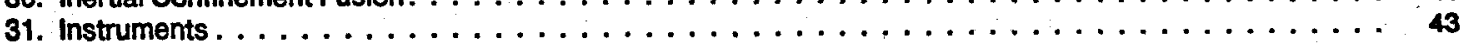

32. Isotope Separation. . . . . . . . . . . . . . . . . . . . . . . 46

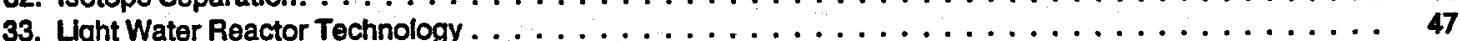

34. Liquid Metal Fast Breeder Reactors: Technology . . . . . . . . . . . . . . . . . . . None

35. LMFBR - Fuels and Materials Engineering and Development:

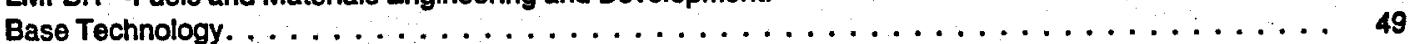

36. LMFBR-Safety Technology . . . . . . . . . . . . . . . . . . . . . 50

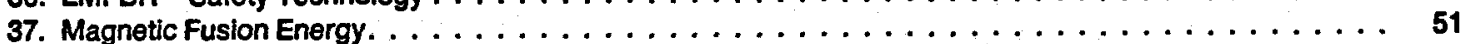

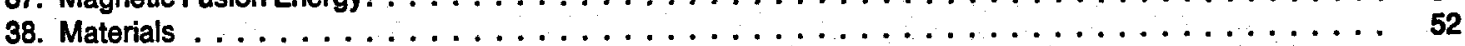

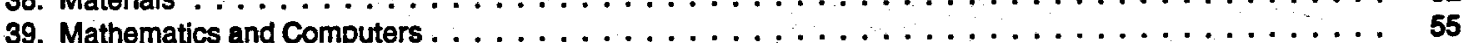

40. Nuclear Propulsion Systems and Aerospace Safety . . . . . . . . . . . . . . . . . 61

41. Nuclear Raw Materials. . . . . . . . . . . . . . . . . . . . . . . . . . . None

42. Nuclear Waste Management . . . . . . . . . . . . . . . . . . . . . . . . . . 62

43. Oil Shales and Tar Sands . . . . . . . . . . . . . . . . . . . . . . . . . . 64

44. Particle Accelerators and High Voltage Machines . . . . . . . . . . . . . . . . . . . . 65

45. Petroleum and Natural Gas . . . . . . . . . . . . . . . . . . . . . . . . . . . . . 69

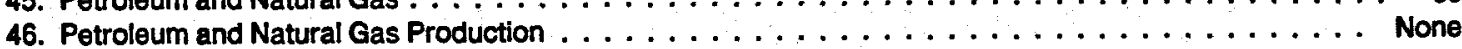

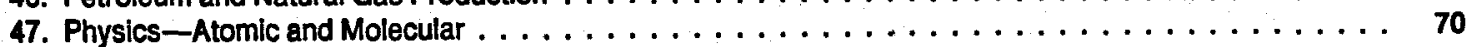

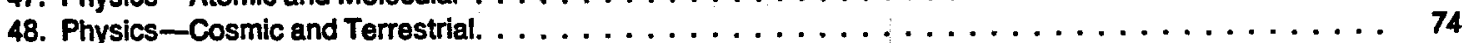

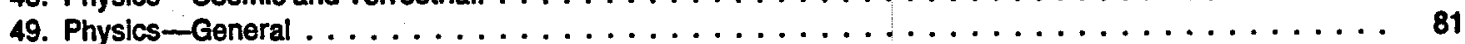


50. Physics-Nuclear $\ldots \ldots \ldots \ldots \ldots \ldots \ldots \ldots \ldots \ldots \ldots \ldots \ldots \ldots \ldots$

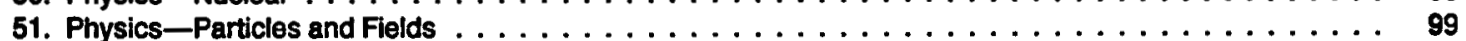

52. Radioistopes and Radiation Applications . . . . . . . . . . . . . . . . . . . 102

53. Safeguards - Nuclear Materials Security . . . . . . . . . . . . . . . . . . . . . . . . . . 103

54. Solar Energy . . . . . . . . . . . . . . . . . . . . . . . . . . . None

55. Solar Thermal. . . . . . . . . . . . . . . . . . . . . . . . . . . . . . . . 107

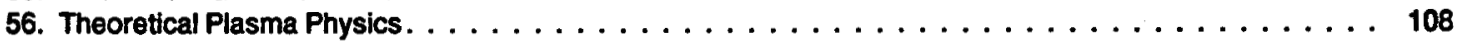

57. Transportation of Property and Nuclear Materials . . . . . . . . . . . . . . . . . . . 109

Author Index $\ldots \ldots \ldots \ldots \ldots \ldots \ldots \ldots \ldots \ldots \ldots$

Numeric Index . . . . . . . . . . . . . . . . . . . . . . . . 155

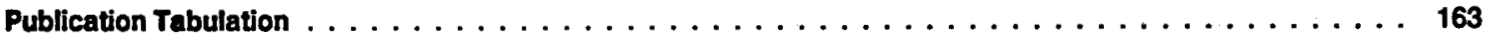




\section{INTRODUCTION}

Los Alamos National Laboratory, which is operated for the US Department of Energy (DOE) by the University of California, was founded in 1943 as part of the wartime Manhattan Project at the site of the Los Alamos Ranch School, a boys' preparatory school in the mountains of northern New Mexico. It had a single assignment-to create the world's first nuclear fission bombs. Success in that task, one of the greatest scientific and technological achievements of all time, was followed by equal success in developing the first thermonuclear weapons. Since 1952, the Laboratory has diversified its programs, and, while still serving as one of the nation's two development centers for nuclear weapons, Los Alamos now devotes about one-half of its total effort to unclassified research, exploring several peaceful applications of nuclear, conventional, and alternative forms of energy. Los Alamos research covers a broad spectrum, ranging from medium- and low-energy nuclear physics research to programs involving medical and blological effects of radiation and basic work in molecular and cellular biology.

Major nonweapons activities at Los Alamos are energy research in fields such as superconducting electrical energy transmission and storage, solar and geothermal energy development, laser fusion research and laser isotope separation, and controlled thermonuclear research using magnetic confinement.
Facilities used in such research at the Laboratory include specialized laboratories; a nuclear reactor designed for a variety of experiments; and particle accelerators such as the 24-MeV, three-stage Van de Graaff particle accelerator and the 27- $\mathrm{MeV}$ electron accelerator designed to produce a very intense, very short burst of $x$ rays for high-speed flash radiography. At the Los Alamos Meson Physics Facility, where physics experiments are carried out and where production of a variety of radioisotopes for medical use is under way, an $800-$ $\mathrm{MeV}, 1-\mathrm{mA}$-beam linear accelerator produces beams of protons, mesons, and other nuclear particles with intensities up to 10000 times greater than those available elsewhere. Another of the Laboratory's facilities, ZT-40, is a toroldal reversed-field pinch device for controlled thermonuclear research involving magnetic confinement of hot plasmas for fusion research. A Central Computing Facility with four Cray-18, two Cray X-MPs, four CDC 7600s, three Cyber 825s, and one Cyber 855 assists in the Laboratory's research.

The Laboratory as of 1984 employed approximately 7500 persons, about one-third of whom are scientists and engineers. The total operating costs of the Laboratory, excluding construction, are about $\$ 640$ million per year. The investment in DOE-owned bulldings and capital equipment at Los Alamos is over $\$ 920$ million. 
The Los Alamos Libraries' continuing program of locating, recording, and announcing publications of Los Alamos-sponsored research has resulted in the previous publications: "Selected Bibliography of Publications of LASL Research, 1957-1962" (LAMS-2895 and supplement), "Publications of LASL Research" for each of the years 1963 through 1966, "Publications of LASL Research, 1967-1971" (LA-5400-MS), "Publications of LASL Research, 1972-1976" (LA-6755-MS, Vols. I and II), "Publications of Los Alamos Research, 1977-1981" (LA-9435-MS, Vols. I and II), "Publications of Los Alamos Research 1982"' (LA-9800-MS), and "Publications of Los Alamos Research 1983" (LA-10155-MS).

This bibliography is a compilation of unclassified publications of work done at Los Alamos National Laboratory for 1984. Publications not included in earlier compilations have also been listed. Declassification of previously classified reports is considered to constitute publication. All classified issuances are omitted-even those papers, themselves unclassified, which were published only as part of a classified document. If a paper was published more than once, all places of publication are Included.

The bibliography includes Los Alamos National Laboratory reports, papers released as non-Laboratory reports, journal articles, books, chapters of books, conference papers either published separately or as part of conference proceedings issued as books or reports, papers published in congressional hearings, theses, and US patents. Publications by Los Alamos authors that are not records of Laboratory-sponsored work are included when the Library becomes aware of them.

The arrangement in each bibliographical citation is title first, place(s) of publication, indented, and authors, further indented. The CHEMICAL ABSTRACTS abbreviation is used for journal citations except for the capitalization. The group designations given after the authors' names indicate the groups with which the authors were associated when the papers were written. Parenthetical information includes report and reprint numbers, book call numbers, and patent numbers. The parentheses Indicate the information by which Los Alamos borrowers may obtain the material from the Los Alamos Libraries.
The entries are arranged in sections by broad subject categories. Within each section they are alphabetical by title. All types of publications are interfiled.

The author and numeric indexes refer to the item numbers, which consist of a number designating the section followed by a dast and a number for the alphabetic arrangement of the item within the section. In the numeric index, the LA- and LA-xxxxMS, etc., numbers of reports are interfiled.

A Keyword-in-Context index included in eartier compilations is not provided.

Most of the reports listed in this publication are available for sale from

National Technical Information Service

US Department of Commerce

Springfield, VA 22151

or on request by government agencies and their contractors from

Office of Scientific and Technical Information

Technical Information Center

US Department of Energy

P.0. Box 62

Oak Ridge, TN 37830

Reprints of some journal articles are available as tong as the supply lasts from the first author listed. Address requests to the first author at

Los Alamos National Laboratory

University of California

P.O. Box 1663

Los Alamos, NM 87545

This bibliography has been prepared by the Los Alamos Library Services Group, IS-4, from the records of the Classification Office, from abstracting and indexing journals, and from supplementary library files. Inquiries regarding any of the materials included should be directed to the Technical Processes Section of the Los Alamos Libraries.

The Libraries gratefully acknowledge the assistance of members of the Laboratory's Administrative Data Processing Division for assistance with the computerized system for handling publication information. 


\section{BIOLOGY AND MEDICINE}

2-1

ALTERATIONS IN THE METABOUSM OF BENZO(A)PYRENE IN SYAIAN HAMSTER EMBRYO (SHE) CELLS PRETREATED WITH PHENOLIC ANTIOXIDANTS.

TIC,

1983. 16P. MN (LA-UR-83-3093)

STRNISTE, GARY F. (LS-3)

OKINAKA, RICHARD T. (LS-3)

CHEN, DAVID JEN-CHI (LS-3)

2-2

ANNUAL REPORT OF THE LIFE SCIENCES

DIVISION, 1983.

LOS ALAMOS,

1984. 117P. (LA-10058-PR)

ENGER, M. DUANE (LS-DO)

STAFFORD, CAROLYN G. (LS-DO)

KARL, SHEILA K. (LS-DO)

23

APPROACH TO SINGLE MOLECULE DETECTION BY LASER - INDUCED FLUORESCENCE.

LASER-BASED ULTRASENSITIVE

SPECTROSCOPY AND DETECTION, 5TH,

1983, SAN DIEGO. PROC., P.71-3.

SPIE, 1983. (621.366/L343BA/1983)

ALSO PUBLISHED IN:

TIC.

1983. 4P. MN (LA-UR-83-2665)

DOVICHI, NORMAN J. (CNC-2)

MARTIN, JOHN C. (LS-4)

JETT, JAMES H. (LS-4)

TRKULA, MITCHELL (CHM-2)

KELLER, RICHARD A. (CHM-2)

$2-4$

ASSIGNMENT OF GENES ENCODING

METALLOTHIONEINS I AND II TO CHINESE HAMSTER CHROMOSOME 3 - EVIDENCE FOR THE ROLE OF CHROMOSOME REARRANGEMENT IN GENE AMPLIFICATION.

MOL. CELL. BIOL.,

V.4,P.2932-5. 1984.

STALLINGS, R.L.

MUNK, A. CHRISTINE (LS-3)

LONGMIRE, JERRY L. (LS-3)

HILDEBRAND, CARL E. (LS-3)

CRAWFORD, BRIAN D. (LS-3)

2-5

ATIEMPTED INDUCTION OF RESISTANCE TO MELPHALAN BY PRETREATING CHINESE HAMSTER CELLS WITH SUBTOXIC DOSES OF THE DRUG.

LOS ALAMOS,

1984. 10P. (LA-10176-MS)

KAYE, M.F.

TOBEY, ROBERT A. (LS-4)
2-6

BIOPHYSICS OF CELL SURFACE MOTILITY. CELL SURFACE DYNAMICS - CONCEPTS AND MODELS, A.S. PERELSON; C. DELISI, F.W. WIEGEL, EDS., P.495-537. DEKKER, 1984.

DEMBO, MICAH (T-10)

HARLOW, FRANCIS H. (T-3)

ALT, WOLFGANG

$2-7$

CARBIB 13-ENRICHED SUBSTRATES FOR IN SITU AND IN VIVO METABOLIC PROFILING

STUDIES BY CARBON 13 NMR.

STABLE ISOTOPES IN NUTRITION,

1983, WASHINGTON. PROC., P.157-73.

ACS, 1984. (612.3/S775IS/1983)

BRAINARD, JAMES R. (INC-4)

HUTSON, JUOITH C (INC-4)

LONDON, ROBERT E. (INC-4)

MATWIYOFF, NICHOLAS A. (INC-DO)

$2-8$

CELL - CELL ADHESION - PARAMETERS AND POSSIBLE MECHANISMS.

CELL SURFACE DYNAMICS - CONCEPTS AND AND MODELS, A.S. PERELSON, C. DELISI, F.W. WIEGEL, EDS., P.459-87.

MARCEL DEKKER, 1984.

BONGRAND, PIERRE

BELL, GEORGE I. (T-DO)

2-9

CELL ADHESION - COMPETITION BETWEEN NONSPECIFIC REPULSION AND SPECIFIC BONDING.

BIOPHYS. J.,

V.45, P.1051-63. 1984.

BELL, GEORGE I. (T-DO)

DEMBO, MICAH (T-10)

BONGRAND, PIERRE

2-10

CELLULAR AUTOMATA IN CYTOSKELETAL

LATTICES.

PHYSICA,

V.10D, P.168-73. 1984

SMITH, STEVEN A. (C-6)

WATT, RICHARD C.

HAMEROFF, STUART R.

2-11

CHANGES IN HISTONE 1 CONTENT AND

CHROMATIN STRUCTURE OF CELLS BLOCKED IN

EARLY S PHASE BY 5-FLUORODEOXYURIDINE

AND APHIDICOLIN.

BIOCHEMISTRY,

V.23, P.5024-8. 1984.

D'ANNA, JOSEPH A. (LS-1)

TOBEY, ROBERT A. (LS-1)
2-12

CHROMOSOME POLYMORPHISM INVOLVING HETEROCHROMATIC BLOCKS IN CHINESE

HAMSTER CHROMOSOME 8.

CYTOGENET. CELL GENET.,

V.38, P.257-64. 1984.

RAY, FRANK A. (LS-4)

BARTHOLDI, MARTY FRANK (LS-4)

KRAEMER, PAUL M. (LS-4)

CRAM, L. SCOTT (LS-4)

2-13

CONSTRUCTION AND ANALYSIS OF DNA

SEQUENCE LIBRARIES FROM FLOW - SORTED

CHROMOSOMES - PRACTICAL AND THEORETICAL CONSIDERATIONS.

NUCLEIC ACIDS RES.

V.12, P.4019-32. 1984

GRIFFITH, JEFFREY KNOWLES (LS-3)

CRAM, L SCOTT (LS-4)

CRAWFORD, BRIAN D. (LS-3)

JACKSON, PAUL J. (LS-4)

SCHILLING, J.

SCHIMKE, R. T

WALTERS, RONALD A. (LS-3)

JETT, JAMES H. (LS-4)

WILDER, MARK E. (LS-4)

2-14

COULTER VOLUME CELL SORTING TO IMPROVE

THE PRECISION OF RADIATION SURVIVAL

ASSAYS.

RADIAT. RES.

V.97, P.608-14. 1984

FREYER, JAMES P. (LS-1)

WILDER, MARK E. (LS-4)

RAJU, MUDUNDI R. (LS-1)

$2-15$

COUNTERSTAINING HUMAN CHROMOSOMES FOR FLOW KARYOLOGY.

CYTOMETRY

V.5, P.580-3. 1984.

MEYNE, JULUANNE (LS-4)

BARTHOLDI, MARTY FRANK (LS-4)

TRAVIS, GAYLE LEE (LS-4)

CRAM, L. SCOTH (LS-4)

2-16

CULTIVATION OF MURINE BONE MARROW MACROPHAGES IN SPONGES - A METHOD THAT PERMITS RECOVERY OF VIABLE CULTURED CELLS.

J. IMMUNOL. METHODS,

V.75, P.149-58. 1984.

AKPORIAYE, EMMANUEL T.

STEWART, S. J. (LS-4)

STEWART, CARLETON (LS-4) 
2-17

DETECTION OF ELEMENTS IN BIOMEDICAL FLUIDS BY LASER - INDUCED BREAKDOWN SPECTROSCOPY.

TIC,

1983. 25P. MN (LA-UR-83-3391)

LOREE, THOMAS R. (CHM-6)

2.18

DIFFUSION LIMITED FORWARD RATE CONSTANTS IN TWO DIMENSIONS - APPLICATION TO THE TRAPPING OF CELL SURFACE RECEPTORS BY COATED PITS

BIOPHYS. J.,

V.46, P.573-86. 1984.

GOLDSTEIN, BYRON (T-10)

GRIEGO, RICHARD

WOFSY, CARLA

2-19

EFFECTS OF CADMIUM ON GLUTATHIONE METABOLISM IN CADMIUM SENSITIVE AND CADMIUM RESISTANT CHINESE HAMSTER CELL LINES.

TOXICOLOGY,

V.29, P.101-7. 1983.

SEAGRAVE, JEANCLARE (LS-3)

HILDEBRAND, CARLE. (LS-3)

ENGER, M. DUANE (LS-3)

2-20

ENZYME REGULATION AND GTP BINDING

PROTEIN - AN ALGORITHM OF CONTROL

THAT INCLUDES PHYSICAL DISPLACEMENT

OF AN INHIBITORY PROTEIN.

ADV. CYCL. NUCL. PROT. PHOS. RES.,

V.16, P.381-90. 1984

YAMAZAKI, AKIO (LS-DO)

UCHIDA, $\mathrm{S}$.

STEIN, PETER J.

WHEELER, GEORGE L. (LS-DO)

BITENSKY, MARK W. (LS-DO)

2-21

EQUILIBRIUM THEORY FOR THE CLUSTERING

OF BIVALENT CELL SURFACE RECEPTORS BY .

TRNALENT LIGANDS - APPLICATION TO

HISTAMINE RELEASE FROM BASOPHILS.

BIOPHYS. $J$.

V.45, P.1109-22. 1984.

GOLDSTEIN, BYRON (T-10)

PERELSON, ALAN S. (T-10)

2-22

EVIDENCE THAT TWO HYDROGEN BONDS COUPLE

2-AMINOPURINE-CYTOSINE BASE MISPAIRS IN

DNA - A POSSIBLE OBSERVATION OF A

DISFAVORED BASE TAUTOMER.

CELLULAR RESPONSES TO DNA DAMAGE,

1983, KEYSTONE. PROC., P.459-70.

A.R. USS, 1983.

(574.873/L393RE/1983)

GOODMAN, MYRONF.

RATLIFF, ROBERT L. (LS-3)
$2-23$

FLOW CYTOMETRIC DETERMINATION OF RADIATION-INDUCED CHROMOSOME DAMAGE AND ITS CORRELATION WITH CELL SUAVIVAL.

RADIAT. RES.

V.99, P.F44-51. 1984.

WELLEWEERD, J.

WILDER, MARK E. (LS-4)

CARPENTER, SUSAN G. (LS-1)

RAJU, MUDUNDI R. (LS-1)

$2-24$

FLOW CYTOMETRIC QUANTIFICATION OF

RADIATION RESPONSES OF MURINE PERITONEAL CELLS.

BIOLOGICAL DOSIMETRY, 1982, MUNICH. PROC., P.265-71. SPRINGER-VERLAG, 1984.

ALSO PUBLISHED IN:

TIC,

1982. 20P. MN (LA-UR-82-3658)

TOKITA, NOBUHIKO (LS-1)

RAJU, MUDUNDI R. (LS-1)

2-25

FLOW CYTOMETRY.

REV. SCI. WSTRUM.,

V.55, P.1375-97. 1984.

STEINKAMP, JOHN A. (LS-4)

2-26

FLOW KARYOLOGY OF SERIALLY CULTURED

CHINESE HAMSTER CELL LINEAGES.

CYTOMETRY,

V.5, P.534-8. 1984.

BARTHOLDI, MARTY FRANK (LS-4)

RAY, FRANK A. (LS-4)

CRAM, L SCOTT (LS-4)

KRAEMER, PAUL M. (LS-4)

2-27

HUMAN VARIATIONS - DELTA CARBON 13 IN ADULT BONE COLLAGEN AND THE RELATION TO

DIET IN AN ISOCHRONOUS CARBON 4 (MAIZE) ARCHAEOLOGICAL POPULATION.

LOS ALAMOS,

1984. 188P. (LA-10259-T)

BUMSTED, M. PAMELA (CHM-1)

2-28

IN SITU OXYGEN CONSUMPTION RATES OF

CELLS IN V - 79 MULTICELLULAR SPHEROIDS

DURING GROWTH.

J. CELL. PHYSIOL.

V.118,P.53-61. 1984.

FREYER, JAMES P. (LS-1)

TUSTANOFF, E.

FRANKO, A.J.

SUTHERLAND, R. $M$.
2-29

VVO STUDIES OF PYRIDINE NUCLEOTIDE

METABOUSM IN ESCHERICHIA COU AND

SACCHAROMYCES CEREVISIAE BY CARBON-13 NMR SPECTROSCOPY.

J. BIOL. CHEM.

V.259, P.2311-20. 1984.

UNKEFER, CLIFFORD, J. (INC-4)

LONDON, ROBERT E. (INC-4)

2-30

INFORMATION STORAGE IN DNA SEOUENCES.

TIC.

1983. 24P. MN (LA-UR-63-1308)

BURKS, CHRISTIAN (T-10)

$2-31$

INSTRUMENT FOR VRUS IDENTIFICATION BY

POLARIZED LGHT SCATTERING - A

PRELIMINARY REPORT.

TIC,

1984. 11P: MN (LA-UR-84-1841)

SALZMAN, GARY C. (LS-DO)

GREGG, CHARLEST. (LS-DO)

$2-32$

KINETICS OF CELL-MEDIATED CYTOTOXICITY STOCHASTIC AND DETERMINISTIC MULTISTAGE MODELS.

MATH. BIOSCl.,

V.70, P.161-94. 1984.

PERELSON, ALAN S. (T-10)

MACKEN, CATHERINEA.

2-33

KINETICS OF ROULEAU FORMATION. II.

REVERSIBLE REACTIONS.

BIOPHYS. J.

V.45, P.805-23. 1984

SAMSEL, RICHARD W.

PERELSON, ALAN S. (T-10)

$2-34$

LIGHT ACTIVATION OF A COMPLEX MIXTURE EFFECTS OF UV EXCISION REPAIR ON THE MODULATION OF GENOTOXIC AND MOLECULAR

EVENTS IN CHINESE HAMSTER CELLS

PHOTOCHEM. PHOTOBIOL.

V.39, P.353-8. 1984.

OKINAKA, RICHARD T. (LS-3)

BINGHAM, JUDY MARIE (LS-3)

MAC INNES, MARK A. (LS-3)

STRNISTE, GARY F. (LS-3)

$2-35$

UGHT ACTIVATION OF GENOTOXIC COMPONENTS IN NATURAL AND SYNTHETIC CRUDE OILS.

LIFE SCIENCES SYMPOSIUM, 5TH, 1982.

GATUNBURG, PROC., P.475-90.

BUTTERWORTH, 1984.

ALSO PUBLISHED IN:

TIC.

1982, 31P, MN (LA-UR-82-3127)

STRNISTE, GARY F. (LS-3) 
286

MECHANISM OF ACTIVATION OF LIGHT. ACTIVATED PHOSPHODIESTERASE AND EVIDENCE FOR HOMOLOGY WITH HORMONEACTIVATED ADENYLATE CYCLASE.

ADV. CYCL. NUCL. PROT. PHOS. RES.,

V.17, P.227-37. 1984.

BITENSKY, MARK W. (LS-DO)

YAMAZAKI, AKIO (LS-DO)

WHEELER, MARCIA A. (LS-DO)

GEORGE, JOHN S. (LS-DO)

RASENICK, MARK M.

2-37

MECHANISM OF CELL-MEDIATED CYTOTOXICITY

AT THE SINGLE CELL LEVEL - VIII.

KINETICS OF LYSIS OF TARGET CELLS

BOUND BY MORE THAN ONE CYTOTOXIC T

LYMPHOCYTE.

J. IMMUNOL

V.132, P.2190-97. 1984

PERELSON, ALAN S. (T-10)

MACKEN, CATHERINEA

GRIMM, ELIZABETH A.

ROOS, LAURIES.

BONAVIDA, BENJAMIN

2.38

MODIFICATION OF DAVYDOV SOLITONS BY THE EXTRINSIC HYDROGEN-NITROGEN-CARBON EQUALS OXYGEN GROUP.

TIC.

1983. 30P. MN (LA-UR-83-2253)

LAYNE, SCOTT P. (CNLS)

$2-39$

MOLECULAR AND SOMATIC CELL GENETIC

ANALYSIS OF METAL RESISTANCE MECHANISMS IN MAMMALIAN CELLS.

TIC,

1983. 20P. MN (LA-UR-63-2677)

ENGER, M. DUANE (LS-DO)

HILDEBRAND, CARLE. (LS-3)

WALTERS, RONALD A. (LS-3)

SEAGRAVE, JEANCLARE (LS-3)

BARHAM, STEVEN S.

HOAGLAND, H. CLARK

2-40

MULTISTAGE MODEL FOR THE ACTION OF

CYTOTOXICTLYMPHOCYTES IN

MULTICELLULAR CONJUGATES.

J. IMMUNOL

V.132,P.1614-24. 1984.

MACKEN, CATHERINE A.

PERELSON, ALAN S. (T-10)

$2-41$

NEWBORN HUMAN SKIN FIBROBLASTS SENESCE IN VITRO WITHOUT ACQUIRING ADULT GROWTH FACTOR REQUIREMENTS.

EXP. CELL RES.

V.154, P.310-4. 1984.

WHARTON, W. R. (LS-4)
2-42

NONLINEAR DYNAMICS OF GLOBULAR PROTEINS.

INTERNATIONAL CONFERENCE ON NONUNEAR

ELECTRODYNAMICS IN BIOLOGICAL SYSTEMS

1983, LOMA LINDA. PROC., P.143-64.

PLENUM, 1984.

ALSO PUBLISHED IN:

TIC,

1983. 13P. MN (LA-UR-63-2252)

LOMDAHL, PETER S. (CNLS)

2-43

NONLINEAR ELECTAODYNAMICS IN

CYTOSKELETAL PROTEIN LATTICES.

TIC,

1983. 18P. MN (LA-UR-83-2431)

HAMEROFF, STUART R.

SMITH, STEVEN A. (C-6)

WATT, RICHARD C.

2-44

PARTICULATE OIL SHALE INHALATION AND

PULMONAFY INFLAMMATORY RESPONSE IN

RATS.

TIC.

1983. 23P. MN (LA-UR-83-3583)

WILSON, JULE S. (LS-1)

HOLLAND, LAURENCE M. (LS-DO)

HALLECK, MARGARET S. (LS-1)

SAUNDERS, GEORGE C. (LS-4)

MARTINEZ, ELMOH. (LS-3)

2-45

PERIODIC STRUCTURALIY SIMILAR OLIGOMERS ARE FOUND ON ONE SIDE OF THE AXES OF SYMMETRY IN THE LAC, TRP, AND GAL OPERATORS.

J. BIOMOL STRUCT. DYN.

V.2, P.387-94. 1984

NUSSINOV, RUTH (T-10)

LENNON, GREGORY G. (T-10)

2-46

PHOTOTRANSFORMATION OF POLYCYCLIC

AROMATIC HYDROCARBONS INTO STABLE, MUTAGENIC COMPONENTS.

TIC.

1983. 13P. MN (LA-UR-83-3110)

OKINAKA, RICHARD T. (LS-3)

NICKOLS, JOYCE W. (LS-3)

WHALEY, THOMAS W. (LS-5)

STRNISTE, GARY F. (LS-3)

2-47

POSSIBLE MECHANISM FOR GENERAL

ANESTHESIA.

LOS ALAMOS SCI.,

SPRING, NO.10, P.23-31. 1984

LAYNE, SCOTT P. (CNLS)

$2-48$

PREPARATION AND CHARACTERIZATION OF LUNG CELL SUSPENSIONS FROM TWO RODENT

SPECIES.

LOS ALAMOS,

1984. 11P. (LA-10179-MS)

WILSON, JULIE S. (LS-1)

LONDON, JERRY E. (LS-1)
2-49

PREPARATION OF HISTONE VARIANTS AND HIGH-MOBILTY GROUP PROTEINS BY REVERSED-PHASE HIGH-PERFORMANCE UQUID CHROMATOGRAPHY.

J. CHROMATOGR.

V.297, P.147-65. 1984.

GURLEY, LAWRENCE R. (LS-1)

D'ANNA, JOSEPH A. (LS-1)

BLUMENFELD, $M$.

VALOEZ, JOSEPH G. (LS-1)

SEBRING, ROBERT, J. (LS-1)

DONAHUE, D. K.

PRENTICE, DAVID A. (LS-1)

SPALL, W. DALE (LS-1)

2-50

PRESERVATION OF FC BETA RECEPTOR

MEDIATED PARTICLE PHAGOCYTOSIS AND

BINDING WITH RAT ALVEOLAR MACROPHAGES

ADHERED TO A PLASTIC SUBSTRATE IN A

SERUM FREE SYSTEM.

IMMUNOL COMMUN.

V.13, P.313-23. 1984.

LEHNERT, BRUCE E. (LS-1)

MORROW, PAULE.

2-51

RADIOFREQUENCY AND MAGNET TECHNOLOGY IN MEDICAL NMR.

TiC,

1984. 6P. MN (LA-UR-84-1122)

FUKUSHIMA, EIICHI (INC-4)

2-52

RAPID MICROBIAL IDENTIFICATION BY

CIRCULAR INTENSITY DIFFERENTIAL

SCATTERING.

TIC.

1984. 28P. MN (LA-UR-84-1796)

GREGG, CHARLES T. (LS-DO)

SALZMAN, GARY C. (LS-DO)

2-53

REGULATION OF MONONUCLEAR

PHAGOCYTE PROUFERATION.

FETICULOENDOTTHELIAL SYSTEM, S. M.

REICHARD AND J. P. FLLKINS, EDS.

P.37-53. PLENUM, 1984.

STEWART, C. C. (LS-4)

2-54

SELECTION, ISOLATION, AND

CHARACTERIZATION OF CADMIUM-RESISTANT

DATURA INNOXIA SUSPENSION CULTURES.

PLANT PHYSIOL.,

V.75, P.914-8. 1984

JACKSON, PAUL J. (LS-4)

ROTH, E. JILL

MC CLURE, PETER R. (LS-6)

NARANJO, CLEOM. (LS-3) 
2-55

SOFT X RAYS AS A TOOL TO INVESTIGATE

RAOIATION - SENSITIVE SITES IN

MAMMALIAN CELLS.

SCIENCE WITH SOFT X-RAYS, 1983,

UPTON. PROC., P.172-9. SPIE, 1984.

(621.361/S416W/1983)

ALSO PUBLISHED IN:

TIC,

1983. 9P. MN (LA-UR-83-3156)

BRENNER, DAVID J. (MP-3)

ZAIDER, MARCO (MP-3)

2-56

SOUTONS IN BIOLOGY.

LOS ALAMOS SCI.

SPRING, NO.10, P.3-21. 1984.

LOMDAHL, PETER S. (CNLS)

LAYNE, SCOTT P. (CNLS)

BIGIO, IRVING J. (CHM-5)

2-57

SOLTONS IN SYNTHETIC AND BIOLOGICAL

POLYMERS.

INTERNATIONAL CONFERENCE ON NONUNEAR

ELECTRODYNAMICS IN BIOLOGICAL SYSTEMS

1983, LOMA LINDA. PROC., P.155-73.

PLENUM, 1984.

ALSO PUBLISHED IN:

TIC,

1983. 22P. MN (LA-UR-84-0429)

BISHOP, ALAN R. (T-11)
2-58

STATISTICAL PENETRATION OF DIFFUSE LIGHT

INTO VEGETATIVE CANOPIES - EFFECT ON

PHOTOSYNTHETIC RATE AND UTILITY FOR

CANOPY MEASUREMENT.

AGRIC. METEOROL.,

V.30, P.327-41. 1984.

GUTSCHICK, VINCENT P. (LS-6)

2-59

TOXICOLOGICALSTUDY OF UNISET

(UV-990-22-1).

LOS ALAMOS,

1984. 5P. (LA-10239-MS)

LONDON, JERRY E. (LS-1)

SMITH, DAVID M. (LS-1)

2-60

TUMORIGENICITY ASSAYS IN NUDE MICE-

ANALYSIS OF THE IMPLANTED GELATIN

SPONGE METHOD.

WORKSHOP ON IMMUNE-DEFICIENT

ANIMALS, 1982, CHEXBRES.

PROC., P.214-9. KARGER, 1984.

ALSO PUBLISHED IN:

TIC,

1982. 16P. MN (LA-UR-82-3146)

KRAEMER, PAUL M. (LS-4)

TRAVIS, GAYLE LEE (LS-4)

SAUNDERS, GEORGE C. (LS-4)

RAY, FRANK A. (LS-4)

STEVENSON, ANITA P. (LS-4)

BAME, KAREN (LS-4)

CRAM, L. SCOTT (LS-4)
2-61

WHAT IS A SOLITON?

LOS ALAMOS SCI.

SPRING, NO.10, P.27-31. 1984.

LOMDAHL, PETER S. (CNLS)

2-62

$X$ - RAY IMAGING OF BIOLOGICAL SPECIMENS. INTERNATIONAL CONFERENCE ON LASERS, 1983, SAN FRANCISCO. PROC., P.635-40.

STS PRESS, 1983.

SOLEM, JOHNDALE C. (T-DO) 


\section{CHEMICAL HIGH EXPLOSIVES}

3-1

APPLICATION OF SLAPPER DETONATOR TECHNOLOGY TO THE DESIGN OF SPECIAL DETONATION SYSTEMS.

TIC,

1984. 29P. MN (LA-UR-84-0391)

MEYERS, W.H. (M-7)

3-2

BURNING TOPOLOGY IN THE SHOCK -INDUCED

REACTION OF HETEROGENEOUS EXPLOSIVES. SHOCK WAVES IN CONDENSED MATTER, 1983, SANTA FE. PROC., P.601-4.

ELSEVIER, 1984.

WACKERLE, JERRY D. (M-1)

ANDERSON, ALLAN B. (M-1)

$3-3$

COMPARISON OF THE SHOCK AND STATIC COMPRESSION CURVES FOR FOUR SOLID EXPLOSIVES.

J. ENERG. MATER.,

V.1, P.275-86. 1983.

DICK, JERRY JOEL (M-3)

$$
\text { 3-4 }
$$

EFFECT OF CRYSTAL ORIENTATION ON SHOCK INITIATION SENSITIVITY OF

PENTAERYTHRITOL TETRANITRATE EXPLOSIVE.

APPL. PHYS. LETT.,

V.44, P.859-61. 1984

DICK, JERRY JOEL (M-3)

3.5

EUTECTIC EXPLOSIVES CONTAINING AMMONIUM NITRATE. FINAL REPORT - OCTOBER 1979

THROUGH SEPTEMBER 1981.

LOS ALAMOS,

1984. 14P. (LA-09973-MS)

STINECIPHER, MARY M. (M-1)

3-6

EVALUATION OF THE FUNDAMENTAL RESEARCH

ON EXPLOSIVES (FRE) PROGRAM.

LOS ALAMOS,

1984. 19P. (LA-10152-MS)

RIVERA, THOMAS (M-1)

RABIE, RONALD L. (M-1)
3-7

HIGH - TEMPERATURE - STABLE DETONATORS.

LOS ALAMOS,

1984. 6P. (LA-10172-MS)

DINEGAR, ROBERT H. (M-T)

3-8

MODEL FOR COMPUTING LATE DETONATIONS INITIATED IN PROPELLANTS BY IMPACT (XDT REACTIONS)

LOS ALAMOS,

1984. 42P. (LA-10270-MS)

KARPP, ROBERT R. (M-4)

3-9

NONPRIMARY - EXPLOSIVE, HOT - WIRE DETONATOR.

LOS ALAMOS,

1984. 7P. (LA-10173-MS)

DINEGAR, ROBERT H. (M-7)

3-10

OIL/HIGH EXPLOSIVE COMPATIBILITY STUDY

SELECTION OF SAFING FLUIDS FOR

DAMAGED EXPLOSIVES ASSEMBLIES.

PROPELLANTS EXPLOS. PYROTECH.,

Y.9, P.7-11. 1984.

HARAIS, BETTY W. (M-1)

3-11

OPTICAL DIAGNOSTICS FOR CONDENSED

PHASE SHOCK - COMPRESSED MOLECULAR SYSTEMS.

TIC,

1983. 69P. MN (LA-UR-83-2847)

SCHMIDT, STEPHEN C. (M-6)

MOORE, DAVID S. (CHM-4)

SHANER, J. W. (M-6)

3-12

SCALE UP OF HIGH EXPLOSIVE 5,7

DINITRO - 1 - PICRYLBENZOTRIAZOLE.

LOS ALAMOS,

1984. 99P. (LA-09961-T)

SANCHEZ, J. A. (M-1)
3-13

SHOCK INITIATION OF DETONATION IN

A DILUTE EXPLOSIVE.

PHYS. FLUIDS,

V.27, P.94-105. 1984.

FICKETT, WILDON (M-3)

3-14

SHORT - DURATION SHOCK INITIATION OF

TRIAMINOTRINITROBENZENE (TATB)

SHOCK WAVES IN CONDENSED MATTER,

1983, SANTA FE. PROC., P.531-4.

ELSEVIER, 1984.

SEITZ, WENDELL LEE (WX-2)

3-15

THREE - DIMENSIONAL COMPUTER MODELING

OF A SHOCK RECOVERY EXPERIMENT.

TIC.

1983. 5P. MN (LA-UR-83-2138)

RABIE, RONALD L. (M-1)

VORTHMAN, JOHN E. (M-1)

DIENES, JOHN K. (T-3)

3-16

THREE - DIMENSIONAL HYDRODYNAMIC HOT-SPOT MODEL APPLIED TO PETN,

HMX, TATB, AND NQ.

LOS ALAMOS.

1984. 38P. (LA-10203-MS)

MADER, CHARLES L (T-14)

KERSHNER, JAMES D. (T-14)

3-17

THREE - DIMENSIONAL MODELING OF INERT METAL - LOADED EXPLOSIVES.

J. ENERG. MATER.,

V.1, P.293-324. 1983.

MADER, CHARLES L (T-14)

KERSHNER, JAMES D. (T-14)

PIMBLEY, GEORGE H. (T-14)

3-18

TOXICOLOGICAL STUDY OF THE HIGH

EXPLOSIVE FORMULATION

IMIDAZO(4,5-D)IMIDAZOLE-2,5(1H,3H)

DIONE, TETRAHYDRO-1,4-DINITRO.

LOS ALAMOS,

1984. 5P. (LA-09999-MS)

LONDON, JERRY E. (LS-1)

SMITH, DAVID M. (LS-1) 


\section{CHEMICAL SEPARATIONS PROCESSES FOR PLUTONIUM AND URANIUM}

4-1

ATOMIC SPECTRUM OF PLUTONIUM.

ATOMIC SPECTRUM OF PLUTONIUM, J.

BLAISE, M. FRED \& R.G. GUTMACHER.

612 P. ARGONNE NATIONAL LABORATORY,

1984. (ANL-83-95)

BLAISE, JEAN

FRED, MARK

GUTMACHER, R. G. (Q-4)
4-2

MATERIALS SCIENCE AND TECHNOLOGY (MST)

DIVISION NUCLEAR MATERIALS PROCESS

TECHNOLOGY GROUP (MST-12) CHEMICAL

PROCESS RESEARCH AND DEVELOPMENT REPORT.

LOS ALAMOS,

1984. 25P. (LA-10061-SR)

CLIFTON, DAVID G. (MST-12)
4-3

NONAQUEOUS PROCESSING METHODS. LOS ALAMOS,

1984. 11P. (LA-10169-MS)

COOPS, M. S.

BOWERSOX, DAVID F. (MST-11) 


\section{CHEMISTRY}

5-1

ACCOMPLSHMENTS AND PROMISE OF

TRANSPLUTONIUM RESEARCH.

PHYS. TODAY,

V.37, NO.3, P.35-41. 1984.

KELLER, O.L.

HOFFMAN, DARLEANE C. (INC-DO)

PENNEMAN, ROBERT A. (INC-4)

CHOPPIN, GREGORY R. (INC-4)

5-2

AIR - STABLE REFERENCE MATERIAL FOR

MEASUREMENT OF THE OXYGEN - TO - METAL (O/M) RATIO OF NUCLEAR FUEL.

TIC.

1983. 6P. MN (LA-UR-83-2613)

HAMMOND, C. F. (CHM-1)

HONNELL, RICHARDE. (MST-6)

REIN, JAMESE. (CHM-1)

5-3

ANALYSIS OF MOLECULAR HYDROGEN

USING SPONTANEOUS RAMAN SPECTROSCOPY.

CONFERENCE ON ANALYTICAL CHEMISTRY

IN ENERGY TECHNOLOGY, 26TH, 1983,

KNOXVILLE. PROC., P.355-6. ELSEVIER,

1984.

VEIRS, KIRK D. (CHM-2)

ROSENBLATT, GERD M. (CHM-DO)

5-4

ANALYTIC RESULTS FOR FINITE SYSTEMS OF RINGED FLORY POLYMERS.

CAN. J. CHEM.,

V.62, P.1262-4. 1984. SPOUGE, JOHN L. (T-10)

- 5-5

ANALYTICAL CAPABILITIES OF RIMS

ABSOLUTE SENSITIVITY AND ISOTOPIC

ANALYSIS.

SYMPOSIUM ON RESONANCE, IONIZATION, MASS SPECTROSCOPY, 1984, KNOXVILLE. PROC., P.91-5. INSTITUTE OF PHYSICS 1984.

ALSO PUBLISHED IN:

TIC,

1984. 6P. MN (LA-UR-84-1325)

NOGAR, NICHOLAS S. (CHM-2)

DOWNEY, STEPHEN W. (CHM-2)

MILLER, CHARLES M. (INC-7)

5-6

APPLICATIONS OF SCANNING ELECTRON

MICROSCOPY TO THE STUDY OF MINERAL

MATTER IN PEAT.

TIC,

1983. 17P. MN (LA-UR-83-2578)

RAYMOND, AOBERT, JR. (ESS-2)

ANDREJKO, MICHAEL J.

BARDIN, S.W. (ESS-2)
5-7

CALCULATED PROPERTIES OF.MATRIX ISOLATED MUONIUM IN SOUD XENON.

J. CHEM. PHYS.,

V.81, P.2400-2. 1984.

ETTERS, RICHARD D. (M-6)

DERBYSHIRE, $M$.

$$
\text { 5-8 }
$$

CHEMISTRY DIVISION-

ORGANIZATIONAL PROFILE.

LOS ALAMOS,

1984. 5P. (LALP-84-041)

CHALLEM, JACK (IS-1)

5-9

COMPARISONS OF CLASSICAL AND QUANTUM DYNAMICS FOR INITIALLY LOCALIZED STATES.

J. CHEM. PHYS.,

V.80, P.5036-48. 1984.

DAVIS, MICHAEL J.

HELLER, ERIC J. (T-12)

5-10

CONSIDERATIONS FOR THE INSTALLATION OF AN INDUCTIVELY COUPLED PLASMA FOR THE ANALYSIS OF RADIOACTIVE SAMPLES.

CONFERENCE ON ANALYTICAL CHEMISTRY IN ENERGY TECHNOLOGY, 26TH, 1983, KNOXVILLE. PROC., P.213-6. ELSEVIER, 1984.

APEL, CHARLES T. (CMB-1)

GALLIMORE, DAVID L. (CHM-1)

5-11

CONSIDERATIONS FOR THE INSTALLATION OF AN INDUCTIVELY COUPLED PLASMA FOR THE ANALYSIS OF RADIOACTIVE SAMPLES.

TIC,

1983. 6P. MN (LA-UR-B3-2905)

APEL, CHARLES T. (CHM-1)

GALLIMORE, DAVD L. (CHM-1)

5-12

CONTROLLED - POTENTIAL - COULOMETAIC

DETERMINATION OF URANIUM AT A

PLATINUM ELECTRODE.

TIC,

1983. 5P. MN (LA-UR-63-2970)

SAPONARA, NIJOLEM. (CHM-1)

JACKSON, DARRYL D. (CHM-1)

6-13

COVERAGE STUDY OF THE SELF-DIFFUSION AND CHEMICAL DIFFUSION CONSTANTS FOR DISORDERED OVERLAYERS OF XENON ON

TUNGSTEN (110).

SURF. SCI.,

V.139, P.478-90. 1984

VALONE, STEVEN M. (CNC-2)

DOLL, JIMMIE D. (CHM-2)
$5-14$

DEPENDENCE OF REACTION ATTRIBUTES, INCLUDING DIFFERENTIAL CROSS SECTIONS AND RESONANCE FEATURES, ON CHANGES IN THE POTENTIAL ENERGY SURFACE FOR THE FLUORINE PLUS DEUTERIUM REACTION.

J. CHEM. PHYS.

V.80, P.246-53. 1984.

WALKER, ROBERT B. (T-12)

BLAIS, NORMAND C. (CHM-2)

TRUHLAR, DONALDG.

5-15

DESOLVATION METHOD FOR ASSESSMENT OF CRYSTALLIZAATION ENERGIES ANDION

CROWDING IN RARE-EARTH PERCHLORATES,

CHLORIDES, AND NITRATES.

INORG. CHEM.,

V.23, P.2430-5. 1984

ONSTOTT, EDWARD I. (CHM-2)

BROWN, LAURA B.

PETERSON, EUGENE J. (CHM-1)

5-16

DISPROPORTIONATION AND POLYMERIZATION OF PLUTONIUM(IV) IN DILUTE AQUEOUS

SOLUTIONS.

MATERIALS RESEARCH SOCIETY SYMPOSIA, 1983, BOSTON. PROC., P.867-72.

ELSEVIER, 1984.

ALSO PUBLISHED IN:

TIC,

1983. 8P. MN (LA-UR-83-3295)

NEWTON, THOMAS W. (INC-2)

RUNDBERG, VIRGINIA L (INC-11)

5-17

EFFECTS OF PHOSPHORIC ACID

CONCENTRATION ON OXYGEN REDUCTION KINETICS AT PLATINUM.

J. ELECTRO. SOC.,

V.131, P.823-8. 1984.

HSUEH, K. L.

GONZALEZ, E.R.

SRINIVASAN, S. (E-11)

CHIN, D. T.

E-18

EFFICIENT FORWARD CONVERSION IN A

RAMAN GENERATOR.

TIC,

1984. 14P. MN (LA-UR-84-0635)

CARLSTEN, JOHN L. (CHM-6)

TEUE, JOHN M. (CHM-6)

WENZEL, ROBERT G. (CHM-6) 
5-19

ELECTROCHEMISTRY AS A BASIS FOR

RADIOCHEMICAL GENERATOR SYSTEMS.

RADIONUCLIDE GENERATORS, NEW SYSTEMS

FOR NUCLEAR MEDICINE APPLICATIONS,

1983, SEATTLE. PROC., P.169-75.

ACS, 1984. (616.07/R129GE/1983)

BENTLEY, GLENN E. (INC-3)

STEINKRUGER, FREDERICK J (INC-3)

WANEK, PHILIP M. (INC-3)

5-20

ELECTROPHILIC RADIOBROMINATIONS OF HIPPURIC ACID - AN EXAMPLE OF THE

UTILITY OF ARYLTRIMETHYLSILANE

INTERMEDIATES.

J. LABELLED COMPD.,

V.21, P.415-27. 1984.

WILBUR, D. SCOTT (INC-3)

SVITRA, ZITA V. (INC-3)

5-21

EQUILIBRIUM PRESSURES OVER THE

SYSTEMS BISMUTH TRISULFATE-

DIBISMUTHMONOXYDISULFATE AND

DISBISMUTHMONOXYDISULFATE-

DIBISMUTHDIOXYMOSULFATE - SLOW

TRANSFORMATION BETWEEN TWO CRYSTALLINE

FORMS OF DIBISMUTHMONOXYDISULFATE.

J. CHEM. PHYS.

V.80, P.3408-19. 1984.

JONES, WESLEY M. (CHM-2)

5-22

EVALUATION OF THE INTRODUCTION OF STABLE

NUCLIDES OF BROMINE INTO HIGH SPECIFIC

ACTIVITY RADIOBROMINATIONS.

J. LABELLD COMPD. RADIOPHARM.,

V.21, P.767-78. 1984.

WILBUR, D. SCOTT (INC-3)

GARCIA, SAMMY R. (INC-5)

ADAM, M.J.

RUTH, T. J.

5-23

EVALUATION OF THE RELIABILTY OF

SEVERAL TECHNIQUES FOR THE MEASUREMENT

OF THE VOLUME FRACTION OF SECOND PHASE

MATERIAL

LOS ALAMOS,

1984. 14P. (LA-10133-MS)

JOHNSON, KAYE ALLAN (MST-13)

5-24

EXPERIMENTAL STUDY OF THE DYNAMICS OF

THE HYDROGEN PLUS DEUTERIUM GOING TO

HYDROGEN DEUTERIUM PLUS DEUTERIUM

REACTION AT COLLUSION ENERGIES OF 0.55

AND 1.30EV.

J. CHEM. PHYS

V.81, P.1298-1313. 1984.

GERRITY, DANIEL P. (CHM-2)

VALENTINI, JAMES J. (CHM-2)

5-25

EXPLOSIVE DOUBLE SALTS AND PREPARATION. PATENTED

NOV. 6, 1984, FILED 1982.

(U.S.PATENT-4,481,048)

CADY, HOWARD H. (M-1)

LEE, KIEN Y. (M-1)
5-26

FLOWING AFTERGLOW STUDY OF URANIUM

TETRABOROHYDRIDE.

CHEM. PHYS. LETT.,

V.112, P.169-72. 1984.

BABCOCK, LUCIA M. (CHM-2)

HERD, CHARLES R.

STREIT, GERALDE. (CHM-2)

5-27

FLOWING AFTERGLOW STUDY OF URANOCENE.

CHEM. PHYS. LETT.

V.112, P.173-5. 1984.

BABCOCK, LUCIA M. (CHM-2)

HERD, CHARLES R.

STREIT, GERALD E. (CHM-2)

5-26

HIGH PRECISION ISOTOPIC ANALYSIS OF

NANOGRAM QUANTITIES OF PLUTONIUM.

LOS ALAMOS,

1984. 12P. (LA-10013-MS)

PERRIN, RICHARD E. (INC-7)

KNOBELOCH, GORDON W. (INC-11)

ARMIJO, VONCILLEM. (INC-11)

EFURD, DEWARD W. (INC-7)

$5-29$

HIGH-RESOLUTION SPECTRA OF THE $1(1,1)$ COMING FROM O(0,0) ROTATIONAL

TRANSITION OF WATER IN ARGON AND

KRYPTON MATRICES.

CHEM. PHYS. LETT.,

V.105, P.547-50. 1984.

FRY, HERBERT A. (NC-4)

JONES, LLEWELLYN H. (NC-4)

SWANSON, BASIL I. (INC-4)

$5-30$

INFRARED LASER SPECTROSCOPY USING A

LONG PATHLENGTH ABSORPTION CELL.

TIC,

1983. 16P. MN (LA-UR-63-1568)

KIM, KYU C. (CHM-4)

BRIESMEISTER, RICHARD A. (CHM-4)

5-31

ISOTOPE AND NUCLEAR CHEMISTRY DIVISION

ANNUAL REPORT FY 1983.

LOS ALAMOS,

1984. 280P. (LA-10130-PR)

HOFFMAN, DARLEANE C. (INC-DO)

HEIKEN, JODY H. (INC-DO)

LINDBERG, HELEN A. (INC-DO)

5-32

LARGE SCALE ISOLATION OF 82 STRONTIUM

FOR GENERATOR PRODUCTION.

RADIONUCLIDE GENERATORS,

NEW SYSTEMS FOR NUCLEAR

MEDICINE APPLICATIONS, 1983,

SEATTLE. PROC., P. 123-34.

ACS, 1984. (616.07/R129GE/1983)

ALSO PUBLISHED IN:

TIC,

1983. 18P. MN (LA-UR-63-0939)

THOMAS, K. E. (INC-3)

BARNES, JOHN W. (INC-3)
$5-33$

LARGE-SCALE ISOLATION OF STRONTIUM

82 FOR GENERATOR PRODUCTION.

RADIONUCLIDE GENERATORS, NEW SYSTEMS

FOR NUCLEAR MEDICINE APPLICATIONS,

1983, SEATTLE. PROC., P.1614-24.

ACS, 1984. (616.07/R129GE/1983)

THOMAS, K. E. (CNC-3)

BARNES, JOHNW. (CNC-3)

5-34

LASER - INDUCED FLUORESCENCE OF FLOWING SAMPLES AS AN APPROACH TO SINGLEMOLECULE DETECTION IN LIQUIDS.

ANAL CHEM.

V.56, P.348-54. 1984.

DOVICH, NORMAN J. (CNC-2)

MARTIN, JOHN C. (LS-4)

JETT, JAMES H. (LS-4)

TRKULA, MITCHELL (CHM-2)

KELLER, RICHARD A. (CHM-2)

5-35

LASER DIAGNOSTICS OF MATERIALS AND

CHEMISTRY.

PURE APPL. CHEM.,

V.56, P.1555-67. 1984

ALSO PUBLISHED IN:

TIC.

1984. 36P. MN (LA-UR-84-0711)

HARTFORD, ALLEN, JR. (CHM-DO)

5-36

LASER PHOTODISSOCIATION OF PLUTONIUM HEXAFLUORIDE AND TRANSIENT PRODUCT

ABSORPTION IN THE VISIBLE.

CHEM. PHYS. LETT.

V.110, P.417-9. 1984

KIM, KYU C. (MST-11)

CAMPBELL, GEORGE M. (MST-11)

5-37

LIMITED TRYPSIN PROTEOLYSIS OF

PHOTORECEPTOR GTP-BINDING PROTEIN

UGHT-AND GTP-INDUCED CONFORMATIONAL CHANGES

J. BIOL. CHEM.

V.259, P.516-25. 1984

HALLIDAY, KAREN R.

STEIN, PETER J.

CHERNOFF, NAOMI

WHEELER, GEORGE L. (LS-DO)

BITENSKY, MARK W. (LS-DO)

5-38

LONG PATH LENGTH TEMPERATURE

CONTROLLED ABSORPTION CELL FOR

SPECTROSCOPIC STUDIES OF RADIOACTIVE

COMPOUNDS.

APPL SPECTROSC.

V.38, P.35-8. 1984

BRIESMEISTER, RICHARD A. (CHM-4)

READ, GARY WILLIS (AP-1)

KIM, KYU C. (CHM-4)

FITZPATRICK, JOHN R. (MST-11) 
5-39

LOW VALENT ACTINIDES AS HYDROGENATION

CATALYSTS.

J. CATAL.

V.85, P.536-7. 1984.

MOODY, DAVID C. (CNC-4)

PAINE, ROBERT T., JR.

5-40

LOW-TEMPERATURE SYNTHESIS OF

PLUTONIUM HEXAFLUORIDE USING DIOXYGEN DIFLUORIDE.

J. AM. CHEM. SOC.,

V.106, P.2726. 1984

MALM, JOHN G.

ELLER, P. GARY (INC-4)

ASPREY, LARNED B. (CNC-3)

5-41

MEASUREMENT OF THE CARBON (A 3 II) AND

CARBON (X I SIGMA PLUS G) DISAPPEARANCE

RATES WITH OXYGEN FROM 298 TO 1300

KELVIN.

CHEMISTRY OF COMBUSTION PROCESSES,

1883, SEATTLE. PROC., P.257-66.

ACS, 1984.

(541.361/C517CO/1983)

BAUGHCUM, STEVEN L. (CHM-4)

OLDENBORG, RICHARD C. (CHM-4)

$5-42$

METHOD OF MAKING FINE-GRAINED

TRIAMINOTRINITROBENZENE.

PATENTED

NOV. 6, 1984, FILED 1983.

(U.S.PATENT-4,481,371)

BENZIGER, THEODORE M. (WX-2)

5-43

METHOD OF PRODUCING COPPER 67.

PATENTED

DEC. 11, 1984, FILED 1983.

(U.S.PATENT-4,487,738)

O'BRIEN, HAROLDA. (INC-3)

BARNES, JOHN W. (INC-3)

TAYLOR, WAYNEA. (INC-3)

THOMAS, K. E. (INC-3)

BENTLEY, GLENN E. (INC-3)

5-44

METHOD OF SEPARATING THORIUM FROM

PLUTONIUM.

PATENTED

JUL 10, 1984, FILED 1982.

(U.S.PATENT -4,459,268)

CLIFTON, DAVID G. (MST-12)

BLUM, THOMAS W. (MST-12)

5-45

MONTE CARLO FOURIER PATH INTEGRAL METHODS IN CHEMICAL DYNAMICS.

J. CHEM. PHYS.,

V.81, P.3536-41. 1984.

DOLL, JIMMIE D. (CHM-2)
5-46

NEW PHASES AND CHEMICAL REACTIONS IN SOLID CARBON MONOXIDE UNDER PRESSURE

J. PHYS. CHEM.,

V.88, P.3176-9. 1984

KATZ, ALLEN I

SCHIFERL, DAVID (M-6)

MLLLS, ROBERT L (P-10)

5-47

ORIENTATIONAL ORDERING AND SITE

STRUCTURE OF SILICON TETRAFLUORIDE

TRAPPED IN RARE GAS SOLIDS.

J. CHEM. PHYS.,

V.81, P.5268-71. 1984.

JONES, LLEWELLYN H. (INC-4)

SWANSON, BASIL I. (INC-4)

EKBERG, SCOTT A. (INC-4)

5-48

ORIENTATIONAL ORDERING AND SITE-

SELECTIVE PHOTOCHEMISTAY OF URANIUM

HEXAFLUORIDE ISOLATED IN ARGON MATRICES

J. PHYS. CHEM.,

V.88, P.1285-6. 1984.

JONES, LLEWELLYN H. (INC-4)

SWANSON, BASIL I. (INC-4)

EKBERG, SCOTT A. (CNC-4)

5-49

ORIGIN OF THE CHEMICAL ELEMENTS

AND THE OKLO PHENOMENON.

REVIEW.

GEOCHIM. COSMOCHIM. ACTA,

V.48, P.601. 1984.

CURTIS, DAVIOE. (INC-7)

6-50

OSCILLATOR STRENGTHS FOR NEUTRAL

ATOMIC URANIUM.

J. OPT. SOC. AM.

V.1B, P.300-5. 1984

ALSO PUBLISHED IN:

LOS ALAMOS CONFERENCE ON OPTICS ' 83 ,

PROC., P. 419-26. SPIE, 1983.

(535/L879OP/1983)

BIENIEWSKI, THOMAS M. (CMB-1)

5-51

PLUTONIUM - 240 HALF LIFE DETERMINED

BY ISOTOPE-DILUTION MASS SPECTROMETRIC MEASUREMENT OF GROWN-IN URANIUM-236.

INT. J. APPL. RADIAT. ISOT.,

V.35, P.163-9. 1984

BECKMAN, RICHARD J. (S-1)

MARSH, SINCLAIR F. (CHM-1)

ABERNATHEY, ROBERT M. (CHM-1)

REIN, JAMES E. (CMB-1)

5-52

PYROCHEMICAL PROCESS FOR EXTRACTING

PLUTONIUM FROM AN ELECTROLYTE SALT.

PATENTED

JUL. 10, 1984, FILED 1982.

(U.S.PATENT-4,459,153)

MULLINS, LAWRENCE J., JR. (MST-13)

CHRISTENSEN, DANA CARL (MST-13)
5-53

QUALTY ASSURANCE FOR ENVIRONMENTAL

ANALYTICAL CHEMISTRY - 1983.

LOS ALAMOS,

1984. 183P. (LA-10115-MS)

GLADNEY, E. S. (HSE-8)

BURNS, COLLEENE. (HSE-8)

PERRIN, DANIEL R. (HSE-B)

ROBINSON, RICHARD D. (H-8)

RAYBOLD, NANCY A. (INC-7)

5-54

QUALITY ASSURANCE FOR ENVIRONMENTAL

ANALYTICAL CHEMISTRY - 1982.

LOS ALAMOS,

1984. 91P. (LA-09950-MS)

GLADNEY, E. S. (HSE-8)

BURNS, COLLEEN E. (HSE-8)

PERRIN, DANIEL R. (HSE-8)

ROBINSON, RICHARD D. (H-8)

KNAB, DARYL (H-B)

5-55

QUANTIFICATION OF ORIENTATIONAL ORDERING OF SULFUR HEXAFLUORIDE IN SOLID XENON

MATRICES.

J. CHEM. PHYS.,

V.80, P.2980-2. 1984.

JONES, LLWELLYN H. (INC-4)

SWANSON, BASIL I. (INC-4)

5-56

QUENCHING OF BACKWARD STIMULATED RAMAN

SCATTERING BY BROADBAND FORWARD RAMAN

RADIATION.

OPT. LETT.,

V.9, P.294-6. 1984

SLATKINE, MICHAEL (CHM-5)

BIGIO, IRVING J. (CHM-5)

5-57

RAPID AND PRECISE ALPHA-PARTICLE

SPECTROMETRY FOR PLUTONIUM MATERIALS

NUCL. INSTRUM. METHODS PHYS. RES.,

V.223, P.528-30. 1984.

MATLACK, GEORGEM. (CHM-1)

BUBERNAK, JOSEPH (CHM-1)

5-58

REACTION OF SULFUR DIOXIDE WITH

TRANSITION-METAL HYDRIDES - SYNTHESIS

AND STRUCTURE OF (MU-HYDROGEN)

OSMIUM 3(CARBON OXIDE)10

(MU-SULFUR DIOXIDE)

ORGANOMETALLICS,

V.3, P.1434-8. 1984.

JARVINEN, GORDON D. (INC-4)

RYAN, ROBERT R. (INC-4) 
5-59

REDUCTION OF SULFUR DIOXIDE BY

BISCYCLOPENTADIENYL METAL DIHYDRIDE

(METAL IS MOLYBDENUM OR TUNGSTEN)

TO BISCYCLOPENTADIENYL METAL

THIOSULFATE AND WATER - MOLECULAR

STRUCTURE AND REACTION WITH ACIDS OF AN

ORGANOMETALLIC MOLYBDENUM-THIOSULFATE

COMPLEX.

INORG. CHEM.,

V.23, P.3181-3. 1984

KUBAS, GREGORY J. (INC-4)

RYAN, ROBERT R. (INC-4)

5-60

RESULTS OF A COOPERATIVE STUDY COMPARING

THE PRECISION OF PEAK HEIGHT AND AREA

MEASUREMENTS IN LIQUID CHROMATOGRAPHY.

J. CHROMATOGR. SCl.,

V.22, P.425-31. 1984.

MC COY, R.W.

AIKEN, R. L.

PAULS, R.E.

ZIEGEL, E. R.

WOLF, T.

FRITZ, GEORGIA T. (M-1)

MARMION, D. M.

5-61

ROLE OF CHLORINE PROMOTERS IN ETHYLENE EPOXIDATION OVER THE SILVER (110) SURFACE.

TIC,

1983. 26P. MN (LA-UR-83-2838)

CAMPBELL, CHARLES TAYLOR (CHM-2)

PAFFETT, MARK THOMAS (E-11)

5-62

ROTATIONAL SPECTRA, MOLECULAR STRUCTURES, AND MERCURY 201 NUCLEAR QUADRUPOLE COUPLING CONSTANTS OF MERCURY HYDROGEN CHLORIDE AND MERCURY DEUTERIUM CHLORIDE

J. CHEM. PHYS.,

V.81, P.5326-34. 1984

SHEA, J. A.

CAMPBELL, E.J.

5-63

SELECTIVE LASER IONIZATION FOR MASS

SPECTRAL ISOTOPIC ANALYSIS.

LASER-BASED ULTRASENSITIVE

SPECTROSCOPY AND DETECTION, 5TH,

1983, SAN DIEGO. PROC., P.8-12.

SPIE, 1983. (621.366/L343BA/1983)

ALSO PUBLISHED IN:

TIC,

1883. 6P. MN (LA-UR-83-2319)

MILLER, CHARLES M. (INC-7)

NOGAR, NICHOLAS S. (CHM-2)

DOWNEY, STEPHEN W. (CHM-2)
$5-64$

SOLUBIUTY OF URANIUM HEXAFLUORIDE

IN SOME LOW - TEMPERATURE SOLVENTS.

J. CHEM. ENG. DATA,

V.29, P.190-3. 1984.

MAIER, WLLIAM B., II (CHM-4)

BEATTE, WLLARD H. (AP-4)

5-65

SOURCE NOISE REDUCTION IN DIODE LASER

SPECTROSCOPY USING THE FARADAY EFFECT.

APPL OPT.,

V.23, P.2155-8. 1984

LAGUNA, GLENN A. (CHM-4)

5-66

SPECTROPHOTOMETRIC METHOD FOR THE

DETERMINATION OF URANIUM IN URINE.

ANAL CHEM.

V.56, P.2269-71. 1984.

KRESSIN, IVAN K. (H-5)

5-67

SPECTROSCOPY OF SOLUTIONS OF URANIUM

(IV) CHLORIDES AT LOW TEMPERATURES

BETWEEN 330 AND 2500 NM.

SPECTROCHIM. ACTA

V.40A, P.897-906, 1984.

BEATTIE, WILLARD H. (CHM-4)

MAIER, WILLIAM B., II (CHM-4)

HOLLAND, REDUS F. (CHM-4)

5-68

STRUCTURAL DETERMINATIONS OF SOME

CHLOROAZEPINE-2,5-DIONES USING A

LANTHANIDE SHIFT REAGENT.

J. HETEROCYCL. CHEM.

V.21, P.801-6. 1984.

WLBUR, D. SCOTT (NC-3)

$5-69$

STRUCTURES AND TRANSITIONS IN SOLID

OXYGEN TO 13 GPA AT $298 \mathrm{~K}$ BY X - RAY

DIFFRACTION.

J. CHEM. PHYS.

V.81,P.5068-73. 1984.

OLINGER, BARTON $W .(M-6)$

MILLS, ROBERT L. (P-10)

ROOF, RAYMOND BRADLEY, JR. (MST

5-70

STUDIES OF IGNITION AND COMBUSTION WAVE PROPAGATION IN EXOTHERMIC CERAMIC

SYSTEMS USING HIGH - SPEED PHOTOGRAPHY. HIGH SPEED PHOTOGRAPHY, VIDEOGRAPHY, AND PHOTONICS, 2ND, 1984, SAN DIEGO.

PROC., P.24-7. SPIE, 1984.

(778.37/H6385P/1984)

MELTON, G. F. (MST-3)

BEHRENS, ROBERT G. (MST-3)

5-71

SURFACE SCIENCE STUDY OF SELECTIVE ETHYLENE EPOXIDATION CATALYZED BY THE SILVER(110) SURFACE - STRUCTURAL SENSITIVITY.

TIC.

1983. 17P. MN (LA-UR-83-2689)

ALSO PUBLISHED IN:

J. VAC. SCI. TECHNOL.

V.A2, P.1024-7. 1984.

CAMPBELL, CHARLES TAYLOR (CHM-2)
5-72

DACE SELF - DIFFUSION CONSTANTS AT

LOW TEMPERATURE - MONTE CARLO

TRANSITION STATE THEORY WITH IMPORTANCE SAMPLING.

J. CHEM. PHYS.

V.80, P.5814-6. 1984

VOTER, AATHUR F. (CHM-2)

DOL, JIMMIE D. (CHM-2)

5-73

SURFACE STRUCTURAL DETERMINATION OF

URANIUM OXYGEN (111) AND (100) USING

RUTHERFORD BACKSCATTERING

J. VAC. SCI. TECHNOL.

V.A2, P.1005-6. 1984.

THOMPSON, KENNETH A. (CHM-2)

VALONE, STEVEN M. (MST-3)

ELUS, WALTON P. (CHM-2)

TAYLOR, THOMAS N. (CHM-2)

MAGGIORE, CARL J. (E-11)

5-74

SYNTHESIS OF OXYGEN - 18 ENRICHED

PHENOL AND NAPHTHOL FROM 18 OXYGEN.

J. LABEUED COMPD.

V.21, P.353-60. 1984

WALKER, THOMAS E. (INC-4)

GOLDBLATT, MAXWELL (CNC-4)

5-75

TEMPERATURE DETERMINATIONS IN THE INDUCTIVELY COUPLED PLASMA USING A

FOURIER TRANSFORM SPECTROMETER.

SPECTROCHIM. ACTA,

V.39B, P.819-28. 1984.

FAIRES, LYNDA M. (CHM-1)

PALMER, BYRONA. (CHM-1)

ENGLEMAN, ROLF, JR. (CHM-1)

NIEMCZYK, THOMASM.

5-76

TERTIARY PHOSPHINE COMPLEXES OF TRIVALENT URANIUM - PREPARATION AND STRUCTURE OF URANIUM, TRIS

[TETRA HYDROBORATE] BIS

[BIS(DIMETHYLPHOSPHINO)ETHANE]

BORON.

J. CHEM. SOC., CHEM. COMMUN.,

V.8, P.532-4. 1984.

WASSERMAN, HARVEY (INC-4)

MOODY, DAVID C. (INC-4)

RYAN, ROBERT R. (INC-4)

5-77

TETRAHYDROBORATE COMPLEXES OF URANIUM WITH 2-(DIPHENYLPHOSPHINO)PYRIDINE.

J. CHEM. SOC., CHEM. COMMUN.,

V.8, P.533-4. 1984.

WASSERMAN, HARVEY (INC-4)

MOODY, DAVID C. (INC-4)

PAINE, ROBERT T., JR.

RYAN, ROBERT R. (INC-4)

SALAZAR, KENNETH V. (INC-4)

5-78

THERMAL DESORPTION OF ARGON AND NEON

FROM SOUD XENON II. STICKING

PROBABILTIES.

J. CHEM. PHYS.,

V.80, P.1681-6. 1984

ADAMS, JOHNE. (CNC-2)

DOLL, JMMMIE D. (CHM-2) 
5-79

THIRD-BODY EFFECTS IN TERMOLECULAR

REACTIONS - HALIDE ION ADDITION TO

BORON TRIFLUORIDE AND BORON TRICHLORIDE.

J. PHYS. CHEM.,

V.88, P.5025-31. 1984

BABCOCK, LUCIA M. (CHM-2)

STREIT, GERALDE. (CHM-2)

$5-80$

TRANSIENT EFFECTS AND PUMP DEPLETION

IN STIMULATED RAMAN SCATTERING.

LOS ALAMOS CONFERENCE ON OPTICS, 1983, LOS ALAMOS AND SANTA FE.

PROC. P.201-6. SPIE, 1983.

(635/L879OP/1983)

ALSO PUBLISHED IN:

TIC,

1983. 8P. MN (LA-UR-83-1851)

CARLSTEN, JOHN L. (CHM-6) WENZEL, ROBERT G. (CHM-6) DRUHL, $K$.

5-81

TRANSITION STATE THEORY DESCRIPTION OF SURFACE SELF - DIFFUSION - COMPARISON WITH CLASSICAL TRAJECTORY RESULTS. J. CHEM. PHYS.,

V.80, P.5832-8. 1984.

VOTER, ARTHUR F. (CHM-2)

DOLL, JIMMIE D. (CHM-2)
5-82

TRITHALLIUM TETRASELENOPHOSPHATE AND

TRITHALLIUM TETRATHIOARSENATE BY

NEUTRON TIME-OF-FLIGHT DIFFRACTION.

ACTA CRYSTALLOGR.,

V.C40, P.1502-6. 1984.

ALKIRE, RANDALL W. (P-8)

VERGAMINI, PHILLIP J. (P-8)

LARSON, ALLEN C. (INC-4)

MOROSIN, BRUNO

5-83

TWO NEW POTENTIAL ENERGY SURFACES

FOR THE FLUORINE PLUS HYDROGEN REACTION.

J. CHEM. PHYS.

V.80, P.232-9. 1984.

TRUHLAR, DONALD G.

GARRETT, B.C.

BLAIS, NORMAND C. (CHM-2)

5-84

UNTANGLING THE WATER GAS SHIFT FROM

FISCHER - TROPSCH - A GORDIAN KNOT? SYMPOSIUM ON CATALYTIC CONVERSION

OF SYNTHETIC GAS AND ALCOHOLS

TO CHEMICALS, 1983, WITHE HAVEN.

PROC., P.97-118. PLENUM, 1984.

ALSO PUBLISHED IN:

TIC.

1983. 68P. MN (LA-UR-83-0260)

ROFER-DE POORTER, CHERYL K. (ESS-2)
5-85

USE OF OXIDES IN THERMOCHEMICAL WATER-SPLITTING CYCLES FOR SOLAR HEAT SOURCES - COPPER OXIDES.

TIC,

1983. 5P. MN (LA-UR-83-2761)

JONES, WESLEY M. (CHM-2)

BOWMAN, MELVIN G. (CMB-3)

5-86

WAVELENGTHS AND ENERGY LEVELS OF DOUBLY

IONIZED URANIUM (U III) OBTAINED

USING A FOURIER-TRANSFORM SPECTROMETER.

J. OPT. SOC. AM.,

V.B1, P.609-25. 1984

PALMER, BYRON A. (CHM-1)

ENGLEMAN, ROLF, JR. (CHM-1) 


\section{COAL CONVERSION AND UTILIZATION-COAL GASIFICATION}

$$
\text { 6-1 }
$$

REVIEW OF VALVE DESIGNS FOR COAL

GASIFICATION LOCKHOPPER SERVICE.

LOS ALAMOS,

1984. 14P. (LA-10024-MS)

CAPPIELLO, CHARLENE C. $(W X-4)$

BENNETT, JOEL G. (Q-13)

GAC, FRANK D. (MST-6) 
4

8. COAL CONVERSION AND UTILIZATION-ENVIRONMENTAL PROTECTION, WASTE UTILIZATION AND SAFETY

8-1

ATTENUATION OF CHEMICAL ELEMENTS IN

ACIDIC LEACHATES FROM COAL MINERAL

WASTES BY SOILS.

LOS ALAMOS,

1984. 14P. (LA-10161-MS)

WANGEN, LAWRENCE E. (CHM-1)

JONES, MARIANNE M. (CHM-1) 


\section{CRITICALITY STUDIES}

9-1

ANALYSIS OF CENTRAL WORTHS AND OTHER INTEGRAL DATA FROM THE LOS ALAMOS BENCHMARK ASSEMBUES.

LOS ALAMOS,

1984. 12P. (LA-10230-MS)

MUIR, DOUGLAS W. (T-2)

9-2

ANALYSIS OF THE YIELD AND QUENCH MECHANISM OF THE ARGENTINE ACCIDENT OF SEPTEMBER 23, 1983.

TRANS. AM. NUCL. SOC.,

V.46, P.460. 1984.

FOREHAND, HARRY M., JR. (Q-2)

9-3

COMPARISON OF CALCULATIONS AND MEASUREMENTS OF NEUTRON LEAKAGE FROM THE LITTLE BOY REPLICA.

TIC.

1984. 6P. MN (LA-UR-83-3630)

FOREHAND, HARRY M., JR. (Q-2)

WHALEN, PAUL P. (X-DO)

MALENFANT, RICHARD E. (Q-2)

9-4

CRITICAL COMMENTARY ON DOSE - RATE

EVALUATIONS.

TIC.

1984. 6P. MN (LA-UR-63-3588)

DOWDY, EDWARD J. (Q-2)

MALENFANT, RICHARD E. (Q-2)

PLASSMANN, EUGENE A. (Q-2)

9-5

CRITICALITY ACCIDENT EXPERIENCEHETEROGENEOUS WATER - MODERATED SYTEMS.

TRANS. AM. NUCL. SOC.,

V.46, P.460-2. 1984.

STRATTON, WILLIAM R. (ITO)

9-6

EVALUATION OF THE 63 COPPER ACTIVATION FOIL FOR DETERMINING THE NEUTRON DOSE IN THE ENERGY RANGE OF $1 \mathrm{EV}$ TO $1 \mathrm{MEV}$. LOS ALAMOS,

1984. 18P. (LA-10074-MS)

EISEN, YOSEF Y. (HSE-1)

VASILIK, DENNIS G. (HSE-1)

BRAKE, RICHARD JOSEPH (HSE-1)
9-7

EXPERIMENTS AT LOS ALAMOS NATIONAL LABORATORY WITH THE REPLICA OF THE HIROSHIMA WEAPON.

US-JAPAN JOINT WORKSHOP FOR

REASSESSMENT OF ATOMIC BOMB RADIATION

DOSIMETRY IN HIROSHIMA AND NAGASAKI

WITH SPECIAL REFERENCE TO SHIELDING

AND ORGAN DOSES, 2ND, 1983.

HIROSHIMA. PROC., P.21-5. RERF, 1984.

(539.77/U84RE/2ND/1983)

WHALEN, PAULP. $(X-D O)$

SORAN, PATRICK D. $(X-6)$

MALENFANT, RICHARD E. (Q-2)

FOREHAND, HARRY M., JR. (Q-2)

9-8

FIELDABLE COMPUTER SYSTEM FOR

DETERMINING GAMMA - RAY PULSE - HEIGHT

DISTRIBUTIONS, FLUX SPECTRA, AND DOSE

RATES FROM LITTLE BOY.

TIC,

1983. 10P. MN (LA-UR-B3-3349)

MOSS, CALVIN E. (Q-2)

LUCAS, MARCIA C. (Q-2)

TISINGER, E. W. (Q-2)

HAMM, MARIANNE E. (Q-2)

9-9

GAMMA - RAY DOSIMETRY MEASUREMENTS

OF THE LITTLE BOY REPLICA.

TIC,

1984. 8P. MN (LA-UR-83-3530)

PLASSMANN, EUGENE A. (Q-2)

PEDERSON, RAYMOND A. (Q-2)

9-10

GAMMA - RAY SPECTRA AND DOSES FROM

THE LITTLE BOY REPLICA.

TIC,

1984. 10P. MN (LA-UR-64-1613)

MOSS, CALVIN E. (Q-2)

LUCAS, MARCIA C. (a-2)

TISINGER, E. W. (Q-2)

HAMM, MARIANNE E. (Q-2)

9-11

LANL CRITICALITY SAFETY AUDITS - GOOD

AND BAD PRACTICES.

TRANS. AM. NUCL. SOC.,

V.46, P.442. 1984.

SMITH, DAVID R. (HSE-6)

MC LAUGHUN, THOMAS P. (HSE-6)
9-12

LITTLE BOY NEUTRON SPECTRUM BELOW

$3 \mathrm{MEV}$.

TIC,

1984. 11P. MN (LA-UR-84-1523)

EVANS, ALBERT E., JR. (Q-2)

BENNETT, E. F.

YULE, T. J.

9-13

LITTLE BOY REPLICATION - JUSTIFICATION AND CONSTRUCTION.

TIC,

1984. 5P. MN (LA-UR-63-3520)

MALENFANT, RICHARDE. (Q-2)

9-14

LITTLE BOY REPLICATION - JUSTIFICATION AND CONSTRUCTION.

TIC,

1984. 5P. MN (LA-UR-64-1726)

MALENFANT, RICHARDE. (S-4)

9-15

NEUTRON DOSIMETRY OF THE LTTLE BOY DEVICE.

TIC,

1984. 7P. MN (LA-UR-83-3593)

PEDERSON, RAYMOND A. (Q-2)

PLASSMANN, EUGENE A. (Q-2)

9-16

NEW INITIATIVES FOR THE LOS ALAMOS

CRITICAL ASSEMBLY FACILITY IN TRAINING, SAFETY, AND DESIGN EVALUATION.

TRANS. AM. NUCL. SOC.

V.46, P.455-6. 1984.

DOWDY, EDWARD J. (Q-2)

9-17

PLUTONIUM BUILDUP IN AND RECOVERY FROM A CAUSTIC WASTE TANK.

TRANS. AM. NUCL. SOC.,

V.46, P.465-6. 1984

MC LAUGHLIN, THOMAS P. (HSE-6)

SMITH, DAVID R. (HSE-6) 
$10-1$

MARKET STUDY OF INTERNATIONAL NEEDS FOR

NEW ELECTRIC GENERATING CAPACITY.

TRANS. AM. NUCL. SOC.,

V.47, P.343. 1984.

MALENFANT, RICHARD E. (S-4) 


\section{ENERGY CONSERVATION-SYSTEMS MODELING AND PERFORMANCE ASSESSMENT}

11-1

ALTERNATIVE GASEOUS FUELS SAFETY ASSESSMENT.

TIC,

1983. 12P. MN (LA-UR-83-3028)

KRUPKA, MILTON C. (5-4)

PEASLEE, A. T. , JR. (S-4)

LAQUER, HENRY L. $(Q-10)$

11-2

DESIGN CONSIDERATIONS FOR VEHICULAR FUEL

CELL POWER PLANTS.

INTERSOCIETY ENERGY CONVERSION

ENGINEERING CONFERENCE, 16TH,

1981, ATLANTA. PROC.,

V.1, P.722-5. ASME, 1981.

(333.7/161EC/1981N.1)

ALSO PUELISHED IN:

TIC,

1981. 14P. MN (LA-UR-81-1054)

LYNN, DAVID K. (E-11)

MC CORMICK, J. BYRON (E-11)

BOBBETT, RONALDE. (E-11)

SRINIVASAN, S. (E-11)

HUFF, JAMES R. (E-11)
11-3

ELECTROCHEMICAL AND SURFACE SCIENCE RESEARCH ON FUEL CELLS, JANUARY 1 DECEMBER 31, 1982.

LOS ALAMOS,

1984. 60P. (LA-10008-PR)

SRINIVASAN, S. (E-11)

GONZALEZ, E. R.

HSUEH, K. L.

CHIN, D. T.

HYDE, P.J. (E-11)

MAGGIORE, CARL J. (E-11)

GOTTESFELD, $S$.

CHANG, H. H.

DEROUIN, CHARLES R. (E-11)

BOBBETT, RONALD E. (E-11)

KORDESCH, K. V.

CARUBELLI, ADEMIR (E-11)

VANDERBORGH, NICHOLAS E. (E-11)

SPRINGER, THOMAS E. (E-11)

NARANJO, RAMONCITA (E-11)
$11-4$

REFUELING CONSIDERATIONS FOR UQUID HYDROGEN FUELED VEHICLES.

TIC.

1984. 32P. MN (LA-UR-84-1490)

STEWART, WALTER F. (P-10) 
6

\section{ENERGY PROJECTIONS AND STATISTICAL INFORMATION}

12-1.

ELECTRICITY UTILITY MARKETS FOR NEW NUCLEAR POWER PLANTS.

TRANS. AM. NUCL. SOC.

V.47, P.342-3. 1984.

JACKSON, SYDNEY V. (S-4)

HARDIE, R. WAYNE (S-4)
12-2

FUTURE MARKET FOR ELECTRIC GENERATING CAPACITY - A SUMMARY OF FINDINGS.

LOS ALAMOS,

1984. 9P. (LALP-84-054)

SUTHERLAND, RONALD J. (S-2)

DRAKE, ROBERT H. (S-2)
12-3

NUCLEAR RATE INCREASE STUDY.

LOS ALAMOS.

1984. 30P. (LA-10099-MS)

DRAKE, ROBERT H. (S-2)

YOUNGBLOOD, ANNETTE G. (S-2)

WILLIAMS, DONNA S. (S-2) 


\section{ENERGY STORAGE}

13-1

SUPERCONDUCTING MAGNETIC ENERGY STORAGE

FOR UTILITY APPLICATIONS.

TIC,

1984. 26P. MN (LA-UR-84-1139)

ROGERS, JOHN D., JR. (CTR-9) 


\section{ENGINEERING AND EQUIPMENT}

14-1

ACCELERATOR APPLICATIONS OF

PHOTOCONDUCTIVE POWER SWITCHES.

LOS ALAMOS,

1983. 30P. (LA-09760-MS)

NUNNALLY, WILLIAM C. (E-11)

14-2

ANALOG QUADRATURE SIGNAL TO PHASE ANGLE

DATA CONVERSION BY A QUADRATURE

DIGITIZER AND QUADRATURE COUNTER.

PATENTED

JAN. 17, 1984, FILED 1981.

(U.S.PATENT-4,426,620)

BUCHENAUER, C. JERALD (CTR-7)

14-3

APPARATUS AND METHOD FOR QUANTITATIVE ASSAY OF GENERIC TRANSURANIC WASTES

FROM NUCLEAR REACTORS.

PATENTED

NOV. 20, 1984, FILED 1982

(U.S.PATENT-4,483,816)

CALDWELL, JOHN T. (Q-2)

KUNZ, WALTER E. (Q-2)

ATENCIO, J.D. (Q-2)

14-4

APPARATUS FOR DETECTING A MAGNETIC

ANOMALY CONTIGUOUS TO REMOTE LOCATION

BY SQUID GRADIOMETER AND MAGNETOMETER SYSTEMS.

PATENTED

MAR. 13, 1984, FILED 1981.

(U.S.PATENT-4,437,064)

OVERTON, WILLIAM C., JR. (P-10)

STEYERT, WILLLAM A., JR. (P-10)

14-5

APPARATUS FOR IRRADIATING A

CONTINUOUSLY FLOWING STREAM OF

FUUID.

PATENTED

AUG. 7, 1984, FILED 1982.

(U.S.PATENT-4,464,330)

SPEIR, LESLIE GAYLE (Q-1)

ADAMS, EDWIN L (Q-1)

14-6

APPLICATION OF OPTICAL - FIBER PINS TO

EXPLOSIVE, PULSE - POWER GENERATORS.

FIBER OPTICS IN ADVERSE ENVIRONMENTS, 2ND, 1984, SAN DIEGO. PROC.,

P.122-6. SPIE, 1984.

(621.369/F443OP/1984)

CAIRD, ROBERT S., JR. (M- - )

BENJAMIN, ROBERT F. (M-6)

MC QUEEN, ROBERT G. (M-6)

ERICKSON, DENNIS J. (M-6)
14-7

BIDIRECTIONAL SLAPPER DETONATOR.

PATENTED

SEPT. 18, 1984, FILED 1982.

(U.S.PATENT-4,471,697)

MC CORMICK, ROBERT NORMAN (M-7)

BOYD, MELISSA D. (M-7)

$14-8$

CAPACITIVELY-COUPLED INDUCTIVE

SENSOR.

PATENTED

MAR. 20, 1984, FILED 1981.

(U.S.PATENT-4,438,394)

EKDAHL, CARL A., JR. (P-1)

14-9

CHARACTERIZÁTION OF FIBER OPTIC CABLES UNDER LARGE TENSILE LOADS.

FIBER OPTICS IN ADVERSE ENVIRONMENTS. 2ND, 1984, SAN DIEGO. PROC.,

P.156-64. SPIE, 1984.

OGLE, J. W. (P-14)

LOONEY, LARRY D. (P-14)

PETERSON, R. T.

14-10

COMPARISON OF REMOTELY ACQUIRED DEEP. BODY AND SUBDERMAL TEMPERATURE MEASUREMENTS FOR DETECTING FEVER IN CATTLE.

nC.

1983. 13P. MN (LA-UR-83-3481)

SEAWRIGHT, GARY L (DAD/AE)

BROWN, R. R. (E-B)

CAMPBELL, KATHERINE (S-1)

LEVINGS, R. L.

ARAKI, CLIFFORD T. (LS-2)

14-11

COMPOSITE POLYMERIC FILM AND METHOD

FOR ITS USE IN INSTALUNG A VERY THIN

POLYMERIC FILM IN A DEVICE

PATENTED

MAY 15, 1984, FILED 1982

(U.S.PATENT-4,448,622)

DUCHANE, DAVID V. (MST-6)

BARTHELL, BARRY L. (MST-6)

14-12

DESIGN AND TESTING OF HIGH-PRESSURE

RAILGUNS AND PROJECTILES.

TIC,

1983. 6P. MN (LA-UR-83-2717)

PETERSON, DENNIS R. $(W X-4)$

FOWLER, CLARENCE M. (M-6)

CUMMINGS, CHARLES E. $(W X-4)$.

KERRISK, JERRY F. $(W X-4)$

PARKER, JERALD V. (P-7)

MARSH, STANLEY P. (M-6)
14-13

DESIGN OF A NEW ELECTROPLATING

LABORATORY

PLAT. SURF. FINISH.

V.71, NO.11, P.20-2. 1984.

MAYER, ANTON (MST-6)

14-14

DEVICE FOR LOADING THIN WIRES IN A

VACUUM.

REV. SCI. INSTRUM.

V.55, P.39-41. 1984

KANIA, DON R. (P-1)

14-15

EDGE - LOADED METAL FLANGE SEAL - ITS

CHARACTERISTICS AND APPLICATIONS.

J. VAC. SCI. TECHNOL.

V.A2, P.1176-9. 1984.

HIGGINS, ROBERT W. (ESS-11)

14-16

EFFECTS OF BLOCKING CONTACTS ON GENERATION-RECOMBINATION NOISE AND RESPONSIVITY IN INTRINSIC

PHOTOCONDUCTORS.

J. APPL. PHYS.

V.56, P.1663-9. 1984.

SMITH, DARRYL L. (E-11)

1417

EFFICIENCY OF A BISMUTH - GERMANATE SCINTILLATOR - COMPARISON OF MONTE CARLO CALCULATIONS WITH MEASUREMENTS.

TIC.

1983. 6P. MN (LA-UR-83-3009)

HSU, H. H. (X-5)

DOWDY, EDWARD J. (Q-2)

ESTES, GUY P. $(\boldsymbol{X}-6)$

LUCAS, MARCIA C. (Q-2)

MACK, JOSEPH M., JR. $(x-6)$

MOSS, CALVIN E. (Q-2)

HAMM, MARIANNE E. (Q-2)

$14-18$

ELECTRICAL AND THERMAL MODEUNG OF

RAILGUNS.

TIC,

1983. 5P. MN (LA-UR-83-2579)

KERRISK, JERRY F. $(\mathbf{X X - 4 )}$

14-19

ELECTRONICS DIVISION RESEARCH AND

DEVELOPMENT, OCTOBER 1, 1982

SEPTEMBER 30, 1983.

LOS ALAMOS,

1984. 193P. (LA-10059-PR)

MAC ROBERTS, MARTIN D. (E-DO)

COURTNEY, ELIZABETH J. (E-DO) 
14-20

EXPERIMENT TO DETERMINE PROPERTIES OF

PACKED PARTICLE BEDS AND REGENERATORS AT CRYOGENIC TEMPERATURES.

ADV. CRYOG. ENG.,

V.29, P.605-12. 1984.

BARCLAY, JOHN A. (P-10)

OVERTON, WILLIAM C., JR. (P-10)

STEWART, WALTER F. (P-10)

SARANGI, SUNIL (P-10)

14-21

EXPERIMENTAL INVESTIGATION OF A

MAGNETIC GATE AS A MULTIMEGAMPERE,

VACUUM OPENING SWITCH.

APPL. PHYS. LETT.

V.44, P.741-3. 1984.

KANIA, DON R. (P-14)

JONES, LARRY A. (P-1)

ZIMMERMANN, EUGENE L. (P-1)

VEESER, LYNN R. (P-14)

TRAINOR, ROBERT JAMES (P-1)

14-22

EXPERIMENTAL TECHNIQUES WITH THE

SPLTT HOPKINSON PRESSURE BAR.

HIGH ENERGY RATE FABRICATION, 1984,

SAN ANTONIO. PROC., P.229-35.

ASME, 1984.

ALSO PUBLISHED IN:

TIC,

1984. 28P. MN (LA-UR-83-3422)

FRANTZ, CHARLES E. (MST-5)

FOLLANSBEE, PAUL S. (MST-5)

WRIGHT, WALTER J. (MST-5)

14-23

EXPERIMENTAL TESTS OF A MOVING FOIL AS

A HIGH CURRENT VACUUM OPENING SWITCH

APPL. PHYS. LETT.

V.45, P.26-8. 1984.

KANIA, DON R. (P-14)

ZIMMERMANN, EUGENE L. (P-1)

TRAINOR, ROBERT JAMES $(P-1)$

VEESER, LYNN R. (P-14)

JONES, LARRY A. (P-1)

14-24

FABRICATION AND LEAK - TIGHT FURNACE

BRAZING OF INTRICATE OBJECTS.

REV. SCI. INSTRUM.

V.55, P.793-5. 1984.

SWIFT, GREG W. $(P-10)$

MIGLIORI, ALBERT (P-10)

WHEATLEY, JOHN C. (P-10)

WALLER, C. R. (MP-8)

SUAZO, G. (MP-8)

14-25

FABRICATION OF A TANTALUM NEUTRAL

SOURCE HEAT EXCHANGER.

WELD. J.,

APRIL, P.43-4. 1984

DIXON, RAY D. (MST-6)

CRANE, HORRIS (CMB-6)

CRISLER, TOMMY L

VIGIL, VICTOR (MST-6)
14-26

FAST PHOTOCONDUCTORS FOR SYNCHROTRON

RADIATION RESEARCH.

NUCL. INSTRUM. METHODS PHYS. RES.,

V.222, P.270-3. 1984.

KANIA, DON R. (P-14)

BARTLETT, ROGER J. (P-14)

WAGNER, RONALD S. (E-11)

HAMMOND, ROBERT B. (E-11)

PIANETTA, PIERO

14-27

FIBER OPTIC SENSOR FOR MEASURING

MULTIMEGAMPERE CURRENTS FROM FLUX

COMPRESSION GENERATORS.

FIBER OPTICS IN ADVERSE ENVIRONMENTS

2ND, 1984, SAN DIEGO. PROC.

P.103-8. SPIE, 1984.

(621.369/F4430P/1984)

VEESER, LYNN R. (P-14)

CAIRD, ROBERT S., JR. (M-6)

FOWLER, CLARENCE M. (M-6)

ERICKSON, DENNIS J. (M-6)

14-28

HEAT COLLECTOR.

PATENTED

MAR. 20, 1984, FILED 1981.

(U.S.PATENT-4,437,456)

MERRIGAN, MICHAEL A. (Q-13)

$14-29$

HIGH EFFICIENCY PHOTOIONIZATION

DETECTOR.

PATENTED

JAN. 31, 1984, FILED 1981.

(U.S.PATENT-4,429,228)

ANDERSON, DAVID F. $(\mathrm{H}-1)$

$14-30$

INDIUM - PHOSPHIDE PICOSECOND

PHOTOCONDUCTORS.

PICOSECOND OPTOELECTRONICS, 1983,

SAN DIEGO. PROC., P.192-6. SPIE.

1983. (621.36/P598OP/1983)

ALSO PUBLISHED IN

TIC,

1983. 6P. MN (LA-UR-83-1897)

HAMMOND, ROBERT B. (E-11)

WAGNER, RONALD S. (E-11)

PAULTER, NICHOLAS G. (E-11)

14-31

INDIUM PHOSPHORUS - IRON

PHOTOCONDUCTING DEVICE.

PATENTED

DEC. 25, 1984, FILED 1982.

(U.S.PATENT-4,490,709)

HAMMOND, ROBERT B. (E-11)

PAULTER, NICHOLAS G. (E-11)

WAGNER, RONALD S. (E-11)

$14-32$

INDUCTIVE STORAGE PULSE CIRCUIT

DEVICE.

PATENTED

SEPT. 25, 1984, FILED 1984

(U.S.PATENT-4,473,875)

PARSONS, W. M. (CTR-9)

HONIG, EMANUEL M. (NSP/AWT)
$14-33$

INFLUENCE OF A PLASMA DURING LASER

WELDING.

TIC.

1983. 30P. MN (LA-UR-83-3701)

DIXON, RAY D. (MST-6)

LEWIS, GARY K. (MST-6)

$14-34$

LECTURE 10 - MEASUREMENT TECHNIQUES TIC,

1980. 42P. MN (LA-UR-80-2272)

WILLIS, WALTER LEE (AP-1)

14-35

LECTURE 3 - PULSE VOLTAGE CIRCUITS.

TIC,

1980. 58P. MN (LA-UR-80-2082)

WILUS, WALTER LEE (AP-1)

14-36

LECTURE 5 - DISCHARGE CIRCUITS AND

LOADS.

TIC.

1980. 61P. MN (LA-UR-80-2271)

SARJEANT, WALTER J. (E-11)

$14-37$

LOSSES IN MAGNETIC FLUX COMPRESSION GENERATORS, PART I. LINEAR DIFFUSION.

LOS ALAMOS,

1984. 61P. (LA-09956-MS, V.I)

FOWLER, CLARENCE M. (M-6)

$14-38$

MAGNETIC AND PHOTOCONDUCTIVE REPETITIVE RAILGUN OPENING SWITCHES.

LOS ALAMOS,

1984. 10P. (LA-09940-MS)

NUNNALLY, WILLIAM C. (E-11)

$14-39$

MAGNETIC REFRIGERATION - THE PROMISE AND THE PROBLEMS

TIC,

1984. 6P. MN (LA-UR-84-0745)

EARCLAY, JOHN A. (P-10)

$14-40$

MAGNETIC REFRIGERATION APPARATUS AND METHOD.

PATENTED

JUL. 17, 1984, FILED 1983.

(U.S.PATENT-4,459,811)

BARCLAY, JOHN A. (P-10)

OVERTON, WILLIAM C., JR. (P-10)

STEWART, WALTER F. (P-10)

$14-41$

MAGNETIC SWITCHES AND CIRCUITS.

LOS ALAMOS

1984. 37P. (LA-08862-MS, REV. 2)

NUNNALLY, WILLIAM C. (E-11) 
$14-42$

MECHANICAL DESIGN ASPECTS OF THE HYVAX RAILGUN.

TIC,

1983. 4P. MN (LA-UR-83-2716)

FOX, WILLE. $W X-4$ )

CUMMINGS, CHARLES E. $(W X-4)$

DAVIDSON, RICH FRANK $(W \times-4)$

PARKER, JERALD V. (P-T)

14-43

METHOD AND APPARATUS FOR PRODUCING

CRYOGENIC TARGETS.

PATENTED

AUG. 7, 1984, FILED 1981.

(U.S.PATENT-4,464,413)

MURPHY, JAMES T. (CMB-10)

MULER, JOHN R. (L-7)

$14-44$

METHOD FOR PRODUCTION OF HYDROCARBONS FROM HYDRATES.

PATENTED

JAN., 10, 1984, FILED 1981.

(U.S.PATENT-4,424,866)

MC GUIRE, PATRICK L. (ESS-3)

14-45

METHOD OF ACCURIZING RAIL GUNS.

LOS ALAMOS,

1984. 11P. (LA-10028-MS)

FARNUM, EUGENE H. (CMB-10)

14-46

METHOD OF FORMING A THIN UNBACKED

METAL FOIL

PATENTED

NOV 13, 1984, FILED 1982

(U.S.PATENT-4,481,999)

DUCHANE, DAVID V. (MST-7)

BARTHELL, BARRY L. (MST-7)

$14-47$

MICROSCOPE AND METHOD OF USE.

PATENTED

APR. 17, 1984, FILED 1981

(U.S.PATENT-4,442,714)

BONGIANNI, W. L. (MST-6)

14-48

MICROSHEUL-TIPPED OPTICAL FIBERS AS

SENSORS OF HIGH-PRESSURE PULSES IN

ADVERSE ENVIRONMENTS.

FIBER OPTICS IN ADVERSE ENVIRONMENTS,

2ND, 1984, SAN DIEGO. PROC.

P.116-20. SPIE, 1984

BENJAMIN, ROBERT F. (M-6)

MAYER, FREDERICK J.

MAYNARD, ROBERT L

14-49

MIRROR FILTER SYSTEMS FOR THE SOFT

$X$ - RAY REGION

NUCL INSTRUM METHODS.

V.222, P.95-8. 1984

BARTLETT, ROGER J. (P-14)

KANIA, DON R. (P-14)

DAY, ROBERT H. (P-14)

KALLNE, ELISABETH (P-1)
$14-50$

NOVEL OPTICAL PIN USING MICROBALLOONS

MATERIALS RESEARCH SOCIETY SYMPOSIA,

1984, ALBANY. PROC., P.299-300.

ELSEVIER, 1984.

ALSO PUBLISHED IN

TIC,

1983. 3P. MN (LA-UR-83-2147)

BENJAMIN, ROBERT F. (M-6)

$14-51$

OPTICAL PUMPING IN A WHISPERING

MODE OPTICAL WAVEGUIDE

PATENTED

MAR. 27, 1984, FILED 1981.

(U.S.PATENT-4,439,860)

KURNIT, NORMAN A. (CHM-6)

$14-52$

PHOTOCONDUCTIVE POWER SWITCHES

LOS ALAMOS

1984. 40P. (LA-09759-MS)

NUNNALLY, WILLIAM C. (E-11)

HAMMOND, ROBERT B. (E-11)

$14-53$

PRESSURE DROP AND FILM HEIGHT

MEASUREMENTS FOR ANNULAR GAS

LQUID FLOW.

INT. J. MULTIPHASE FLOW,

V.10, P. $341-56.1984$

LAURINAT, J. E.

HANRATTY, T. J.

DALLMAN, JOHN C. (M-3)

14-54

PRINCIPLES AND PROSPECTS FOR MICRO

HEAT PIPES.

TIC,

1984. 9P. MN (LA-UR-84-0120)

OTTER, THEODORE P. (T-DOT)

14-55

PROGRESS IN DEVELOPING REPETITIVE

PULSE SYSTEMS UTILIZING INDUCTIVE

ENERGY STORAGE.

IEEE PULSED POWER CONF., 4TH, 1983.

ALBUQUERQUE. PROC., P.129-32.

$1983 ?$

ALSO PUBLISHED IN:

TIC.

1983. 5P. MN (LA-UR-83-1585)

HONIG, EMANUEL M. (E-11)

14-56

PURGED WINDOW APPARATUS UTILIZINC

HEATED PURGE GAS

PATENTED

APR. 17, 1984, FILED 1982.

(U.S.PATENT-4,443,072)

BALLARD, EVAN O. (P-12)
14-57

RADIATION INDUCED TRANSIENT

ATTENUATION OF PCS FIBER.

OPTICAL FIBERS IN ADVERSE

ENVIRONMENTS, 1983, PARIS.

PROC., P.68-75. SPIE, 1984.

ALSO PUBLISHED IN

TIC.

1983. 17P. MN (LA-UR-83-1314)

LYONS, PETER B. (P-14)

LOONEY, LARRY D. (P-14)

14-58

REINJECTION LASER OSCILLATOR AND

METHOD.

PATENTED

AUG. 28, 1984, FILED 1981

(U.S. PATENT-4,468,776)

MCLELLAN, EDWARD J. (AP-5)

14-59

REPETITIVE PULSE GENERATION WITH

INDUCTIVE ENERGY STORAGE, A NEW

TECHNOLOGY FOR DEFENSE

LOS ALAMOS,

1984. 5P. (LA-10004)

HONIG, EMANUEL M. (E-11)

$14-60$

STANDARD OPERATING PROCEDURE-

GAS - ATMOSPHERE BRAZING FACILITY/LAMPF

EOUIPMENT TEST LABORATORY

LOS ALAMOS

1984. 55P. (LA-10231-SOP)

SUAZO, G. (MP-B)

KOCZAN, STEVEN P. (ESS-6)

14-61

STEPPING MOTOR CONTROLLER

PATENTED

AUG. 7, 1984, FILED 1982.

(U.S.PATENT-4,464,616)

BOURRET, STEVEN C. (Q-1)

SWANSEN, JAMES E. (Q-1)

$14-62$

SUBNANOSECOND RELATIVE TIMING SYSTEM

AND CABLE MEASUREMENTS ON THE ATRISCO

EVENT.

LOSALAMOS,

1984. 27P. (LA-10031-MS)

WILKE, MARK D. (P-15)

ALRICK, KEITHR. $(P-15)$

EGDORF, $\mathbf{S}$.

14-63

TEST TECHNIQUES FOR MODEL DEVELOPMENT OF REPETITIVE SERVICE ENERGY STORAGE

CAPACITORS.

TIC,

1984. BP. MN (LA-UR-83-3634)

THOMPSON, MELVIN C. (E-8)

MAULDIN, G. H. 
$14-64$

TWO - DIMENSIONAL MODELING OF HIGH EXPLOSIVE DRIVEN PLASMA OPENING SWITCHES.

J. APPL. PHYS.

V.57,P.78-81. 1984

GREENE, ARTHUR E. (X-2)

BOWERS, RICHARD L. (X-2)

14-65

UPDATE ON PRECISION MACHINING AT

LOSALAMOS.

CONTEMPORARY METHODS OF OPTICAL MANUFACTURING AND TESTING, 1983,

SAN DIEGO. PROC., P.107-11. SPIE,

1983. (681.4/C761 ME/1983)

ALSO PUBLISHED IN:

TIC,

1983. 8P. MN (LA-UR-83-2438)

RHORER, RICHARD L. (MEC-4)
$14-66$

WIDE RANGE RADIOACTIVE GAS

CONCENTRATION DETECTOR.

PATENTED

APR. 3, 1984, FILED 1981.

(U.S.PATENT-4,441,024)

ANDERSON, DAVD F. (H-1)

14-67

240-KA SWITCH WITH POTENTIAL APPLICATION IN ELECTROMAGNETIC-LAUNCH SYSTEMS.

ELECTROMAGNETIC LAUNCH TECHNOLOGY, 2ND, 1983, BOSTON. PROC.

P.111-4. IEEE, 1983.

(629.475/S989EL/2ND/1983)

ALSO PUBLISHED IN:

TIC,

1983. 5P. MN (LA-UR-83-2710)

HONIG, EMANUEL M. (E-11)
$14-68$

80 - MW PHOTOCONDUCTOR POWER SWITCH. APPL. PHYS. LETT.,

V.44, P.980-8. 1984

NUNNALLY, WILLLAM C. (E-11)

HAMMOND, ROBERT B. (E-11) 


\section{ENVIRONMENT CONTROL TECHNOLOGY AND EARTH SCIENCES}

16-1

CALDERA DEVELOPMENT DURING THE MINOAN

ERUPTION, THIRA, CYCLADES, GREECE.

J. GEOPHYS. RES.,

V.89, P.8441-62. 1984.

HEIKEN, GRANT H. (ESS-1)

MC COY, FLOYD J.

$16-2$

COPPER SMELTERS AND ATMOSPHERIC

VISIBILITY IN THE SOUTHWEST, SEASONAL

ANALYSIS.

J. AIR POLLUT. CONTROL ASSOC.,

V.34, P.750-1. 1984.

ALSO PUBLISHED IN:

TiC,

1983. 36P. MN (LA-UR-83-0636)

NOCHUMSON, DAVID H. (S-4)

WILLIAMS, MICHAEL D. (S-4)

$16-3$

COPPER SMELTERS AND ATMOSPHERIC

VISIBILITY IN NATIONAL PARKS AND

WILDERNESS AREAS IN THE SOUTHWEST.

LOS ALAMOS,

1984. 87P. (LA-10095-MS)

NOCHUMSON, DAVID H. (S-4)

WILUAMS, MICHAEL D. (S-4)

16-4

CRITERION FOR QUASIBRITTLE CRACK GROWTH.

TIC,

1984. 11P. MN (LA-UR-64-0829)

MARGOLIN, LEONARD G. (ESS-5)

SMITH, BARHAM W. (ESS-5)

16-6

DETAILED MAGNETOTELLURIC/

AUDIOMAGNETOTELLURIC STUDY OF THE

JEMEZ VOLCANIC ZONE, NEW MEXICO.

J. GEOPHYS. RES.,

V.89B, P.3335-62. 1984.

ANDER, MARKE. (ESS-3)

GOSS, RON

STRANGWAY, DAVID

16-6

DUST PARTICLES IN HIGH-SPEED FLOWS

CALCULATIONS OF SMALL PARTICLE

RE-ENTRY HYDRODYNAMICS.

LOS ALAḾOS,

1984. 20P. (LA-10025)

SANDFORD, MAXWELL T. (ESS-5)

16-7

EARTHQUAKE CATALOG FOR NORTHERN

NEW MEXICO, JULY - SEPTEMBER 1982.

LOS ALAMOS,

1984. 11P. (LA-10313-PR)

CASH, DANIEL J. (ESS-3)

OLSEN, KENNETH H. (ESS-3)

STEWART, JOHN N., JR. (ESS-3)

WOLFF, JOYCE J. (ESS-3)
16-8

EARTHQUAKE CATALOG FOR NORTHERN

NEW MEXICO, OCTOBER - DECEMBER 1983.

LOS ALAMOS,

1984. 14P. (LA-10314-PR)

CASH, DANIEL J. (ESS-3)

OLSEN, KENNETH H. (ESS-3)

STEWART, JOHN N., JR. (ESS-3)

WOLFF, JOYCE J. (ESS-3)

16-9

EARTHQUAKE CATALOG FOR NORTHERN NEW

MEXICO, JULY - SEPTEMBER 1983

LOS ALAMOS,

1984. 10P. (LA-10033-PF)

CASH, DANIEL J. (ESS-3)

OLSEN, KENNETH H. (ESS-3)

STEWART, JOHN N., JR. (ESS-3)

WOLFF, JOYCE J. (ESS-3)

16-10

EROSION OF EARTH COVERS USED IN

SHALLOW LAND BURIAL AT LOS ALAMOS,

NEW MEXICO.

J. ENVIRON. QUAL,

V.13, P.361-6. 1984.

NYHAN, JOHN W. (LS-6)

DEPOORTER, G. L.

DRENNON, BARRY J. (LS-6)

SIMANTON, J. R.

FOSTER, GEORGE $R$.

16-11

ESTIMATE OF NEPTUNIUM 237 PRODUCTION

DURING ATMOSPHERIC TESTING.

HEALTH PHYS.

V.47, P.786-7. 1984.

EFURD, DEWARD W. (NNC-7)

KNOBELOCH, GORDON W. (INC-11)

PERIRIN, RICHARD E. (INC-7)

BARA, DONALD W. (INC-11)

16-12

EVALUATION OF VAGINAL IMPLANTS FOR

MULEDEER.

J. WILDL. MANAGE.,

V.48, P.646-8. 1984.

GARROTT, R. A. (LS-6)

GARTMANN, RICHARDM.

16-13

FALLOUT PREDICTION AS OF 1957.

LOS ALAMOS,

1984. 19P. (LA-09993-MS)

STOPINSKI, ORIN W. (NSP/TO)
16-14

GEOCHEMICAL APPLICATIONS OF NUCLEAR MICROPROBES.

NUCL. INSTRUM. METHODS,

V.B3, P.671-6. 1984.

ROGERS, PAMELA S. Z. (INC-7)

DUFFY, CLARENCE J. (INC-11)

BENJAMIN, TIMOTHYM. (INC-7)

MAGGIORE, CARL J. (E-11)

16-15

HARPER - DORN CREEP - AN ARTIFACT OF

LOW-AMPLTTUDE TEMPERATURE CYCLING?

GEOPHYS. RES. LETT.,

V.11, P.117-20. 1984.

WEERTMAN, JOHANNES (MST-5)

BLACIC, JAMES D. (ESS-3)

16-16

HUGONIOT DATA FOR PYRRHOTITE AND THE

EARTH'S CORE.

J. GEOPHYS. RES.

V.89B, P.6041-6. 1984.

BROWN, J. MICHAEL

AHRENS, THOMAS J.

SHAMPINE, DENNIS L (M-6)

16-17

HYDRAULIC TESTING OF CRUSHED

BANDELER TUFF.

LOS ALAMOS,

1984.21P. (LA-10037-MS)

ABEELE, WILLYY. (LS-6)

16-18

HYDRODYNAMIC ASPECTS OF CALDERA-

FORMING ERUPTIONS - NUMERICAL MODELS.

J. GEOPHYS. RES.,

V.89, P. B269-85. 1984.

WOHLETZ. KENNETH H. (ESS-1)

MC GETCHIN, THOMAS R.

SANDFORD, MAXWELL T. (ESS-5)

JONES, ERIC M. (ESS-5)

16-19

HYDROLOGIC CHARACTERISTICS OF THE MAIN AQUIFER IN THE LOS ALAMOS AREA-

DEVELOPMENT OF GROUND WATER SUPPLIES. LOS ALAMOS,

1984. 44P. (LA-09957-MS)

PURTYMUN, WILLIAM D. (HSE-8)

16-20

IMPROVED APPARATUS AND TECHNIOUES FOR HIGH-TEMPERATURE AND PRESSURE DEFORMATION OF CRYSTALLINE MATERIALS.

LOS ALAMOS,

1984. 21P. (LA-10182-MS)

KOLLE, J.J.

BLACIC, JAMES D. (ESS-3) 
$16-21$

LA MESA FIRE SYMPOSIUM. LOS ALAMOS,

NEW MEXICO, OCTOBER 6 AND 7, 1981.

LOS ALAMOS,

1984. 172P. (LA-09236-NERP)

FOXX, TERALENE S. $(\mathrm{H}-8)$

16-22

UDAR MEASUREMENTS IN THE VICINITY OF

THE KINGSTON STEAM PLANT, TENNESSEE,

DURING MARCH 18 - 22, 1981. LOS ALAMOS,

1984. 92P. (LA-10086-MS)

HORAK, HENRY G. (ESS-7)

WHITAKER, RODNEY W. (ESS-5)

KARL, ROBERT RAYMOND, JR (ESS-7)

ARGO, HAROLDV. (ESS-9)

16-23

MEASUREMENTS OF STRATOSPHERIC BROMINE

J. GEOPHYS. RES.,

V.89D, P.4821-5. 1984

SEDLACEK, WIULAM A. (INC-7)

LAZRUS, ALLAN L

GANORUD, B. W.

16-24

MODEL FOR THE TECTONIC DEVELOPMENT OF

THE SOUTHEASTERN COLORADO PLATEAU

BOUNDARY

J. GEOPHYS. RES.,

V.89, P.10,207-17. 1984

ALDRICH, MERRITT J. (ESS-1)

LAUGHLIN, A. WILLIAM (ESS-2)

16-25

OBSERVED RATE OF TROPOSPHERIC DIFFUSION

LOS ALAMOS

1984. 14P. (LA-10181-MS)

GIFFORD, F. A

16-26

ORIGIN OF A ROBERTS VICTOR SANIDINE-

COESITE GROSPYDITE - THERMODYNAMIC

CONSIDERATIONS.

INTERNATIONAL KIMBERUTE CONFERENCE,

PROC., P.33-42. ELSEVIER, 1984.

3RD, 1984, CLERMONT-FERRAND.

ALSO PUBLISHED IN:

TIC,

1982. 24P. MN (LA-UR-82-3641)

WOHLETZ, KENNETH H. (ESS-1)

SMYTH, JOSEPH R. (ESS-1)

$16-27$

PHYSIOLOGIC RESPONSE OF ELK TO

DIFFERENCES IN WINTER RANGE QUALITY.

J. WILDL. MANAGE.

V.48, P.248-53. 1984.

WEBER, BRUCE J.

WOLFE, MICHAELL

WHITE, GARY C. (LS-6)

ROWLAND, MARYM.

16-28

PLASTICITY ANO HYDROLYTIC WEAKENING OF

QUARTZ SINGLE CRYSTALS.

J. GEOPHYS. RES.,

V.89B, P.4223-37. 1984.

BLACIC, JAMES D. (ESS-3)

CHRISTIE, J.M.
16-29

PREDICTION OF SOIL LOSS WITH THE

CREAMS MODEL

TIC,

1984. 10P. MN (LA-UR-84-1399)

BECKER, NAOMI M. (HSE-8)

$16-30$

PRELIMINARY RESULTS OF EROSION AND

CONTAMINANT TRANSPORT RESEARCH ON THE NEVADA TEST SITE.

TIC,

1984. 15P. MN (LA-UR-84-1000)

BOSTICK, KENNETH V. (LS-6)

SIMANTON, J. R.

LANE, LEONARD J. (LS-6)

HAKONSON, THOMAS E. (LS-6)

$16-31$

PROCEEDINGS OF WORKSHOP ON ATMOSPHERIC RESEARCH NEEDS FOR THE WESTERN

UNITED STATES - SPONSORED BY

LOS ALAMOS NATIONAL LABORATORY,

LOS ALAMOS, NEW MEXICO, MARCH 13-15, 1984.

LOS ALAMOS,

1984. 34P. (LA-10109-C)

YAMADA, TETSUNI (ESS-5)

STREIT, GERALDE. (CNC-2)

NOCHUMSON, DAVID H. (S-4)

MROZ, EUGENE J. (INC-7)

CLEMENTS, WILLIAM E. (ESS-7)

WILLIAMS, MICHAEL D. (S-4)

$16-32$

PRODUCE AND FISH SAMPLNG PROGRAM OF

LOS ALAMOS NATIONAL LABORATORY'S

ENVIRONMENTAL SURVEILLANCE GROUP.

LOS ALAMOS,

1984. 8P. (LA-10186-MS)

SALAZAR, JOHN (H-8)

$16-33$

RADIATIVE HEAT TRANSFER IN MOLTEN AND

GLASSY OBSIDIAN.

J. GEOPHYS. RES.,

V.89B,P.7107-10. 1984.

GABLE, CARL

SHANKLAND, THOMAS J. (ESS-3)

$16-34$

REGIONAL AIR QUALITY IN THE FOUR

CORNERS STUDY REGION.

J. AIR POLLUT. CONTROL ASSOC.

V.33, P.670-7. 1983

NOCHUMSON, DAVID H. (S-4)

$16-35$

SEARCH FOR IRIDIUM ABUNDANCE ANOMALIES

AT TWO LATE CAMBRIAN BIOMERE BOUNDARIES

IN WESTERN UTAH

SCIENCE,

V.223, P.163-5. 1984

ORTH, CHARLES J. (INC-11)

KNIGHT, JERE D. (INC-11)

QUINTANA, LEONARD R. (INC-11)

GILMORE, JAMES S. (CNC-11)

PALMER; A. R.
$16-36$

SOME PHYSICAL CONCEPTS IN COMPLEX

TERRAIN METEOROLOGY DERIVED FROM THE

U. S. DEPARTMENT OF ENERGY'S ASCOT

PROGRAM.

TIC,

1984. 44P. MN (LA-UR-84-0808)

CLEMENTS, WILLIAM E. (ESS-7)

16-37

STATISTICAL EXPLORATION OF THE EFFECTS

OF COPPER SMELTER EMISSIONS ON

VISIBILITY IN SOUTHWESTERN NATIONAL

PARKS.

TIC,

1984. 17P. MN (LA-UR-84-1035)

WLLIAMS, MICHAEL D. (\$-4)

NOCHUMSON, DAVD H. (S-4)

$16-38$

STATUS OF THE FLORA OF THE LOS ALAMOS

NATIONAL ENVIRONMENTAL RESEARCH PARK,

A HISTORICAL PERSPECTIVE.

LOS ALAMOS,

1984. 50P. (LA-08050-NERP V.2)

FOXX, TERALENE S. (HSE-8)

TIERNEY, GAIL D. (HSE-B)

16-39

SUBSURFACE TRANSPORT OF CONTAMINANTS FROM ENERGY PROCESS WASTE LEACHATES, JANUARY 1 - DECEMBER 31, 1982.

LOS ALAMOS,

1984. 12P. (LA-10011-PR)

WANGEN, LAWRENCE E. (LS-6)

STALLINGS, E. A. (LS-6)

$16-40$

TESTING OF LATERAL WATER FLOW IN A

MOISTURE BARRIER.

LOS ALAMOS

1984. 4P. (LA-10125-MS)

ABEELE, WILLYV. (LS-6)

DE POORTER, GERALD L. (LS-6)

$16-41$

TOWING TANK STUDIES OF STRATIFIED FLOW

OVER RIDGES ANO VALLEYS.

CONFERENCE ON MOUNTAIN METEOROLOGY, 3RD, 1984, PORTLAND. PROC., P.37-41.

AMS, 1984.

LEE, JAMES T., JR. (ESS-7)

BARR, SUMNER (ESS-7)

LAWSON, ROBERT E., JR.

SNYDER, WILLIAM H.

MARSH, G.L.

16-42

TRACE ELEMENT PATTERNS AT A NON-MARINE CRETACEOUS - TERTIARY BOUNDARY.

NATURE,

V.307, P.224-8. 1984

GILMORE, JAMES S. (CNC-11)

KNIGHT, JERE D. (INC-11)

ORTH, CHARLES J. (INC-11)

PILLMORE, C. L

TSCHUDY, R. H. 
$16-43$

USE OF PELLET - GROUP PLOTS TO MEASURE TRENDS IN DEER AND ELK POPULATIONS.

WILDL. SOC. BULL.

V.12, P.147-55. 1984.

ROWLAND, MARY M.

WHITE, GARY C. (LS-6)

KARLEN, E. M. (LS-6)
16-44

WATER SUPPLY AT LOS ALAMOS DURING 1982.

LOS ALAMOS,

1984. 46P. (LA-09896-PA)

PURTYMUN, WILLIAM D. (HSE-8)

BECKER, NAOMI M. (HSE-8)

MAES, MAX N. (HSE-8)
16-45

WAVE PROPAGATION IN INELASTIC MEDIA.

INTL. CONF. OF THE CENTER FOR

NONLINEAR STUDIES, 3RD, 1983,

LOS ALAMOS. PROC., P.163-70.

NORTH-HOLLAND PHYSICS PUBL., 1984. (530.15/161CE/3RD/1983)

MARGOLIN, LEONARD G. (ESS-5) 


\section{EXPERIMENTAL PLASMA PHYSICS}

$17-1$

ANALYSIS OF FLUCTUATING SIGNALS FROM A MULTICHORD INTERFEROMETER.

J. APPL. PHYS.,

V.56, P.757-73. 1984

RUSBRIDGE, MICHAEL G.

JACOBSON, ABRAM R. (CTR-2)

$17-2$

COAXIAL SLOW SOURCE - A QUASI - STATIC

FRC FORMATION CONCEPT.

TIC,

1984. 5P. MN (LA-UR-84-0603)

MC KENNA, KENNETH F. (CTR-3)

GRIBBLE, ROBERT F. (CTR-4)

$17-3$

COUPUNG OF ENERGY AND FLUX LOSSES

IN FIELD - REVERSED CONFIGURATIONS.

COMM. PLASMA PHYS. CONTROLLED FUSION,

V.8, P.237-42. 1984.

TUSZEWSKI, M. (CTR-3)

$17-4$

CURRENT SENSING IN MAGNETIC FUSION

EXPERIMENTS FOR FARADAY ROTATION IN

SINGLE-MODE OPTICAL FIBERS.

TIC,

1983. 8P. MN (LA-UR-83-3480)

CHANDLER, GEORGE I. (CTR-8)

JAHODA, FRANZ C. (CTR-B)

$17-5$

DIAGNOSTICS OF A HIGH CURRENT CAPILLARY DISCHARGE.

TIC,

1984. 6P. MN (LA-UR-84-1249)

KANIA, DON R. (P-14)

JONES, LARRY A. (P-1)

$17-6$

ELECTRICAL DESIGN OF A HIGH CURRENT

DENSITY AIR - CORE REVERSED - FIELD

PINCH ZTP.

TIC,

1983. 5P. MN (LA-UR-63-3490)

REASS, WILLIAM A. (CTR-4)

MELTON, JIMMY G. (CTR-4)

GRIBBLE, ROBERT F. (CTR-4)

17-7

ELECTRON TEMPERATURE MEASUREMENTS IN THE

FIELD-REVERSED CONFIGURATION

EXPERIMENT FRX - C.

NUCL. FUSION,

V.24, P.177-81. 1984.

REJ, DONALD J. (CTR-3)

ARMSTRONG, WILLIAM T. (CTR-3)
17-8

EVALUATION OF LMMTER CONFIGURATIONS

IN ZT - 4OM.

J. NUCL MATER.,

V.128-9, P.517-23. 1984

DOWNING, JAMES N., JR. (CTR-7)

BUCHENAUER, C. JERALD (CTR-7)

INGRAHAM, JOHN C. (CTR-2)

KRAKOWSKI, ROBERT A. (CTR-12)

PHILLIPS, JAMES A. (CTR-DO)

WATT, ROBERT G. (CTR-7)

17-9

EXPERIMENTAL STUDY OF THE EQUILIBRIUM OF FIELD - REVERSED CONFIGURATIONS.

COMM. PLASMA PHYS. CONTROULED FUSION,

V.26, P.991-1005. 1984.

TUSZEWSKI, M. (CTR-3)

17-10

F-O PUMPING AND FIELD MODULATION

EXPERIMENTS ON A REVERSED FIELD PINCH

DISCHARGE.

PHYS. FLUIDS,

V.27, P.548-51. 1984.

SCHOENBERG, KURT F. (CTR-4)

BUCHENAUER, C. JERALD (CTR-7)

MASSEY, ROBERT S. (CTR-1)

MELTON, JIMMY G. (CTR-4)

MOSES, RONALD W., JR. (CTR-6)

NEBEL, R. A. (CTR-6)

PHILUPS, JAMES A. (CTR-DO)

17-11

HIGH DENSITY Z - PINCH RESEARCH.

TIC,

1983. 5P. MN (LA-UR-83-2311)

SHLACHTER, JACK S. (CTR-DOT)

17-12

INITIATION AND ACCRETION EXPERIMENTS

ON THE HDZP.

TIC.

1984. 9P. MN (LA-UR-84-1076)

SHLACHTER, JACK S. (CTR-DOT)

HAMMEL, JAY E. (CTR-DO)

SCUDDER, DAVID W. (CTR-DOT)

17-13

INTERACTION OF THE NEUTRAL DEUTERIUM

FLUX WITH A FIELD - REVERSED

CONFIGURATION.

TIC,

1984. 5P. MN (LA-UR-84-0601)

REJ, DONALD J. (CTR-3)
17-14

INTERNAL MAGNETIC FIELD MEASUREMENTS IN A TRANSLATING FIELD - REVERSED

CONFIGURATION

TIC.

1984. 5P. MN (LA-UR-84-0870)

ARMSTRONG, WIULAM T. (CTR-3)

CHRIEN, ROBERT E. (CTR-3)

MC KENNA, KENNETH F. (CTR-3)

REJ, DONALD J. (CTR-3)

SHERWOOD, EUGENE G. (CTR-11)

SIEMON, RICHARDE. (CTR-3)

TUSZEWSKI, M. (CTR-3)

17-15

INTERPRETATION OF END-ON INTERFEROMETRY

IN FIELD-REVERSED CONFIGURATIONS.

PHYS. FLUIDS,

V.27, P.1058-60. 1984.

TUSZEWSKI, M. (CTR-3)

MC KENNA, KENNETH F. (CTR-3)

$17-16$

LOCALIZED PLASMA - DENSITY DISTURBANCE

PROPAGATING ALONG BETA IN THE INTERIOR

OF THE ZT-4OM REVERSED-FIELD PINCH.

APPL. PHYS. LETT.

V.45, P.34-6. 1984.

NALESSO, GIANFAANCO

JACOBSON, ABRAM R. (CTR-2)

17-17

MODEL OF ENERGY CONFINEMENT IN THE

REVERSED FIELD PINCH.

COMM. PLASMA PHYS. CONTROLLED FUSION,

V.26, P.1119-25. 1984.

MILLER, GUTHRIE (CTR-2)

17-18

NONLOCAL DC ELECTRICAL CONDUCTIVITY

OF A LORENTZ PLASMA IN A STOCHASTIC

MAGNETIC FIELD.

PHYS. REV.

V.29A, P.3335-42.1984.

JACOBSON, ABRAM R. (CTR-2)

MOSES, RONALD W., JR. (CTR-6)

17-19

OPTICAL FEEDBACK WAVELENGTH RESPONSE

AND INTERFERENCE EFFECTS OF SELF-

PUMPED PHASE CONJUGATION IN BARIUM

TITANIUM OXYGEN.

OPT. LETT.

V.9,P.362-4. 1984.

JAHODA, FRANZ C. (CTR-8)

WEBER, PAUL G. (CTR-8)

FEINBERG, $J$. 
1720

OSCILLATING FIELD CURRENT DRIVE FOR REVERSED FIELD PINCH DISCHARGES.

J. APPL PHYS.,

V.56, P.2519-29. 1984.

SCHOENBERG, KURT F. (CTR-4)

GRIBBLE, ROBERT F. (CTR-4)

BAKER, DON A. (CTR-2)

17-21

PARAMETERS OF A POSSIBLE FRC ADIABATIC COMPRESSION EXPERIMENT.

TIC,

1984. 5P. MN (LA-UR-84-0583)

TUSZEWSKI, M. (CTR-3)

SIEMON, RICHARD E. (CTR-3)

17-22

PLASMA RESISTIVITY IN THE PRESENCE

OF A REVERSED - FIELD PINCH DYNAMO.

PHYS. FLUIDS,

V.27, P.1671-5, 1984.

SCHOENBERG, KURT F. (CTR-4)

MOSES, RONALD W., JR. (CTR-6)

HAGENSON, RANDY L.

17-23

POLARIZED RADIAL MAGNETIC FIELDS AND OUTWARD PLASMA FLUXES. DURING SHALLOW REVERSAL DISCHARGES IN THE ZT - 40M REVERSED - FIELD PINCH.

J. APPL. PHYS.,

V.65, P.125-37. 1984.

JACOBSON, ABRAM R. (CTR-2)

RUSBRIDGE, MICHAEL G.

BURKHARDT, LOUIS C. (CTR-2)

17-24

POLOIDAL FIELD SYSTEM DESIGN FOR THE

ZT - H REVERSED FIELD PINCH EXPERIMENT.

TIC.

1983. 7P. MN (LA-UR-63-3476)

SCHOENBERG, KURT F. (CTR-4)

GRIBBLE, ROBERT F. (CTR-4)

LINTON, THEODORE W. (CTR-4)

REASS, WILLIAM A. (CTR-4)

$17-25$

POSSIBLE PLASMA - DYNAMO MECHANISM

DRIVEN BY PARTICLE TRANSPORT.

PHYS. FLUIDS

V.27, P.7-9. 1984

JACOBSON, ABRAM R. (CTR-2)

$17-26$

RECENT RESULTS ON FRC TRANSPORT.

TIC.

1984. 5P. MN (LA-UR-84-0584)

TUSZEWSKI, M. (CTR-3)
$17-27$

RESISTIVE EVOLUTION OF GENERAL PLASMA CONFIGUAATIONS.

LOS ALAMOS.

1984. 28P. (LA-10178-MS)

MILLER, GUTHRIE (CTR-2)

$17-28$

RFP ENERGYEVOLUTION AND CONTAINMENT

IN THE PRESENCE OF GIANT SAWTEETH.

PHYS. FLUIOS,

V.27, P.784-6. 1984.

WATT, ROBERT G. (CTR-7)

LITTLE, EDWARD M. (CTR-7)

17-29

ROLE OF MAGNETIC RECONNECTION PHENOMENA

IN THE REVERSED - FIELD PINCH.

MAGNETIC RECONNECTION IN SPACE AND

LABORATORY PLASMAS, 1983, LOS ALAMOS.

PROC., P.332-9. AGU, 1984

(538.766/M196RE/1983)

ALSO PUBLISHED IN

Tic,

1983. 35P. MN (LA-UR-84-0714)

BAKER, DON A. (CTR-2)

$17-30$

SOFT X - RAY ARRAY RESULTS ON

THE ZT-4OM REVERSED-FIELD PINCH

PHYS. FLUIDS,

V.27, P.551-4. 1984

WURDEN, GLEN A. (CTR-8)

$17-31$

STUDY OF LIMITER DAMAGE IN A MAGNETIC

FIELD ERROR REGION OF THE TT - 4OM

EXPERIMENT.

TIC,

1983. 6P. MN (LA-UR-83-2972)

MAKOWITZ, HENRY

$17-32$

SUSTAINMENT OYNAMO REEXAMINED

NONLOCAL ELECTRICAL CONDUCTIVITY OF

PLASMA IN A STOCHASTIC MAGNETIC FIELD.

TIC,

1984. 5P. MN (LA-UR-84-0524)

JACOBSON, ABRAM R. (CTR-2)

MOSES, RONALD W., JR. (CTR-6)

17.33

SUSTAINMENT DYNAMO REEXAMINED-

NONLOCAL ELECTRICAL CONDUCTIVITY OF

A PLASMA IN A STOCHASTIC MAGNETIC

FIELD.

PHYS. REV. LETT.,

V.52, P.2041-3. 1984

JACOBSON, ABRAM R. (CTR-2)

MOSES, RONALOW. JR. (CTR-6)
$17-34$

TOROIDAL EQUILIBRIUM IN AN IRON CORE

REVERSED-FIELD PINCH.

NUCL. FUSION

V.24, P.789-94. 1984.

MILLE, GUTHRIE (CTR-2)

$17-35$

TOROIDAL MAGNETIC FIELD SYSTEM FOR A

2 - MA REVERSED FIELD PINCH EXPERIMENT.

TIC.

1983. 7P. MN (LA-UR-63-3489)

MELTON, JIMMY G. (CTR-4)

LINTON, THEODORE W. (CTR-4)

$17-36$

TRANSLATION OF FIELD - REVERSED CONFIGURATIONS IN THE FRX - C $/ T$

EXPERIMENT.

TIC.

1984. 6P. MN (LA-UR-84-0602)

REJ, DONALD J. (CTR-3)

ARMSTRONG, WILLIAM T. (CTR-3)

CHRIEN, ROBERT E. (CTR-3)

KLINGNER, PHILLIP L. (CTR-5)

LINFORD, RULON K. (CTR-DO)

MC KENNA, KENNETH F. (CTR-3)

MILROY, RICHARD D.

SHERWOOD, EUGENE G. (CTR-11)

SIEMON, RICHARD E. (CTR-3)

TUSZEWSKI, M. (CTR-3)

$17-37$

TWO NOVEL PLASMA DIAGNOSTICS.

TIC.

1984. 5P. MN (LA-UR-84-0923)

JAHODA, FRANZ C. (CTR-8)

$17-38$

ZERO - DIMENSIONAL TRANSPORT MODEL FOR

FIELD - REVERSED CONFIGURATIONS.

PHYS. FLUIDS,

V.27, P.1514-20. 1984.

REJ, DONALD J. (CTR-3)

TUSZEWSKI, M. (CTR-3) 


\section{FUSION SYSTEMS}

18-1

ANALYSIS OF SURFACE CURRENTS ON THE CTX MESH FLUX CONSERVER.

TIC,

1984. 5P. MN (LA-UR-84-0623)

WRIGHT, BRADFORD L. (CTR-5)

18-2

COMPACT REVERSED-FIELD PINCH REACTORS

(CRFPR) - PRELIMINARY ENGINEERING CONSIDERATIONS.

LOS ALAMOS.

1984. 405P. (LA-10200-MS)

HAGENSON, RANDY L.

KRAKOWSKI, ROBERT A. (CTR-12)

BATHKE, CHARLES G. (CTR-12)

MILER, RONALD L (CTR-12)

EMBRECHTS, MARK J. (CTR-12)

SCHNURR, NORMAN M. (WX-4)

BATTAT, MORRIS E.

LABAUVE, RAPHAEL J.(T-2)

DAVIDSON, J. WILEY (S-4)

18-3

CONCEPTUAL DESIGN FOR AN AIR CORE 2

MEG - AMP REVERSED FIELD EXPERIMENT.

TIC. .

1983. 5P. MN (LA-UR-83-3452)

HAMMER, CHARLES F. (CTR-4)

$18-4$

CONFINEMENT TME AND ENERGY BALANCE

IN THE CTX SPHEROMAK.

TIC,

1984. 6P. MN (LA-UR-84-0620)

BARNES, CHRISTOPHER W. (CTR-5)

HENHS, IVARS (CTR-5)

HOIDA, HIROSHI W. (CTR-5)

JARBOE, THOMAS R. (CTR-5)

18-5

DESIGN AND CONSTRUCTION DETAILS OF THE

FRX - C/T DEVICE - A COMPACT TOROID

PLASMA TRANSLATION EXPERIMENT.

LOS ALAMOS,

1984. 15P. (LA-10108-MS)

REJ. DONALD J. (CTR-3)

18-6

EXPERIMENTAL RESULTS FROM HYDROGEN/

DEUTERIUM DISTILLATIONS AT THE

TRITIUM SYSTEMS TEST ASSEMBLY.

FUSION TECHNOL.

V.6, P.625-8, 1984.

SHERMAN, ROBERT H. (MST-3)

BARTLIT, JOHN R. (MST-3)

VEIRS, KIRK D. (CHM-2)
18-7

IMPROVED ACTIVATION CROSS SECTIONS FOR

VANADIUM AND TITANIUM.

TIC,

1983. 7P. MN (LA-UR-83-2567)

MUIR, DOUGLAS W. (T-2)

ARTHUR, EDWARD D. (T-2)

18-8

MIXED MODE OPERATION IN CTX.

TIC,

1984. 4P. MN (LA-UR-84-0626)

HOIDA, HIROSHI W. (CTR-5)

BARNES, CHRISTOPHER W. (CTR-5)

HENINS, IVARS (CTR-5)

JARBOE, THOMAS R. (CTR-5)

18-9

OHMIC HEATING OF A SPHEROMAK TO $100 \mathrm{EV}$.

PHYS. FLUIDS,

V.27,P.13-5. 1984

JARBOE, THOMAS R. (CTR-5)

BARNES, CHRISTOPHER W. (CTR-5)

HENINS, IVARS (CTR-5)

HOIDA, HIROSHI W. (CTR-5)

KNOX, STEPHEN O. (CTR-5)

LNFORD, RULON K. (CTR-DO)

SHERWOOD, ARTHUR R. (CTR-5)

$18-10$

OHMIC HEATING TO 100 - EV AND HELICITY

INJECTION N CTX.

TIC,

1984. 5P. MN (LA-UR-B4-0621)

HENINS, IVARS (CTR-5)

BARNES, CHRISTOPHER W. (CTR-5)

HOIDA, HIROSHI W. (CTR-5)

JARBOE, THOMAS R. (CTR-5)

KLINGNER, PHILLIP L (CTR-5)

KNOX, STEPHEN O. (CTR-5)

PLATTS, D. A. (CTR-5)

SHERWOOD, ARTHUR R. (CTR-5)

WRIGHT, BRADFORD L. (CTR-5)

18-11

PLASMA ENGINEERING DESIGN OF A COMPACT REVERSED - FIELD PINCH REACTOR (CRFPR).

TIC,

1983. 9P. MN (LA-UR-83-3505)

BATHKE, CHARLES G. (CTR-12)

EMBRECHTS, MARK J. (CTR-12)

HAGENSON, RANDY L.

KRAKOWSKI, ROBERT A. (CTR-12)

MILLER, RONALD L. (CTR-12)

18-12

RADIATION TRANSPORT, APRIL 1, 1983 -

DECEMBER 31, 1983.

LOS ALAMOS

1984. 89P. (LA-10189-PR)

O'DELL, R. DOUGLAS $(X-6)$
18-13

SPACE RESOLVED ABSOLUTELY CALIBRATED

VUV SPECTROSCOPIC MEASUREMENTS ON CTX

TIC,

1k984. 6P. MN (LA-UR-84-0622)

HOIDA, HIROSHI W. (CTR-5)

BARNES, CHRISTOPHER W. (CTR-5)

HENINS, NARS (CTR-5)

JARBOE, THOMAS R. (CTR-5)

SHERWOOD, ARTHUR R. (CTR-5)

18-14

STELLARATOR HELICAL VACUUM VESSEL

TIC.

1983. 4P. MN (LA-UR-83-3439)

YOVORNIK, EDWARD J. (CTR-4)

18-15

STRATEGIC ROLE OF REACTOR SYSTEMS

STUDIES FOR ALTERNATIVE FUSION

CONCEPTS.

TRANS. AM. NUCL. SOC.,

V.46, P.180-1. 1984.

KRAKOWSKI, ROBERT A. (CTR-12)

18-16

SUBSYSTEM COST DATA FOR THE TRITIUM SYSTEMS TEST ASSEMBLY.

TIC,

1983. 8P. MN (LA-UR-83-3415)

BARTLT, JOHN R. (MST-3)

ANDERSON, JAMES L. (MST-3)

REXROTH, VERNER G. (MST:7)

18-17

SUSTAINED SPHEROMAK TECHNOLOGY.

TIC,

1984. 4P. MN (LA-UR-84-0624)

PLATTS, D. A. (CTR-5)

SHERWOOD, ARTHUR R. (CTR-5)

JARBOE, THOMAS R. (CTR-5)

LINFORD, RULON K. (CTR-DO)

HOIDA, HIROSHI W. (CTR-5)

HENINS, IVARS (CTR-5)

18-18

USE OF THE STREAMING MATRIX HYBRID

METHOD FOR DISCRETE ORDINATES FUSION REACTOR CALCULATIONS.

TRANS. AM. NUCL. SOC.,

V.46,P.275-7, 1984.

ALSO PUBLISHED IN

TIC,

1984. 7P. MN (LA-UR-84-0170)

BATTAT, MORRISE.

DAVIDSON, J. WILEY (S-4)

DUDZIAK, DONALD J. (S-4)

THAYER, GARY R. (S-4) 


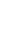

20. GEOTHERMAL ENERGY-ENVIRONMENTAL RESEARCH

20-1

ENVIRONMENTAL STUDIES CONDUCTED AT THE FENTON HOT DRY ROCK GEOTHERMAL

DEVELOPMENT SITE.

LOS ALAMOS,

1984. 19P. (LA-09967-MS)

MIERA, FELIX R., JR. (LS-6)

LANGHORST, GARY J. (LS-6)

MC ELLIN, S. (LS-6)

MONTOYA, CONSUELO (LS-6)
20-2

POTENTIAL EFFECTS OF HYDROGEN SULFIDE

GAS FROM GEOTHERMAL ENERGY CONVERSION

ON TWO PLANT SPECIES NATIVE TO NORTHERN NEW MEXICO.

LOS ALAMOS,

1984. 116P. (LA-09984-T)

GONZALES, GILBERT JOE (LS-6) 


\section{GEOTHERMAL ENERGY-EXPLORATION TECHNOLOGY}

21-1

CORRELATION OF WIRELINE LOG CHARACTERISTICS WITH HYDROTHERMAL ALTERATION AND OTHER RESERVOIR PROPERTIES OF THE SALTON SEA AND WESTMORLAND GEOTHERMAL FIELDS, IMPERIAL VALLEY, CALLFORNIA, USA LOS ALAMOS.

1984. 100P. (LA-10128-MS) MURAMOTO, FRANKS.

ELDERS, WILFRED A.

21-2

DOWNHOLE SEISMIC MONITORING OF AN ACID TREATMENT IN THE BEOWAWE GEOTHERMAL FIELD.

TIC.

1984. 6P. MN (LA-UR-84-1106)

BATRA, RAVI (ESS-3)

ALBRIGHT, JAMES N. (ESS-4)

BRADLEY, CHRISTOPHER C. (CTR-5)

21-3

FRACTURING OPERATIONS IN A DRY GEOTHERMAL RESERVOIR.

TIC,

1983. 35P. MN (LA-UR-83-2300)

ROWLEY, JOHN C. (ESS-4)

PETTITT, ROLAND A. (ESS-4)

HENDRON, ROBERT H. (ESS-4)

SINCLAIR, A. RICHARD

NICHOLSON, ROBERT W.

$21-4$

HOT DRY ROCK GEOTHERMAL RESERVOIR FRACTURING INITIAL FIELD OPERATIONS

1982.

TIC.

1983. 8P. MN (LA-UR-83-1593)

ROWLEY, JOHN C. (ESS-4)

PEITIT, ROLAND A. (ESS-4)

MATSUNAGA, ISAO

DREESEN, DONALD N. (ESS-4)

NICHOLSON, ROBERT W.

SINCLAIR, A. RICHARD
$21-5$

HYDROGEOCHEMISTRY OF THE QUALIBOU CALDERA GEOTHERMAL SYSTEM, ST. LUCIA WEST INDIES.

TIC,

1984. 8P. MN (LA-UR-84-1208)

GOFF, FRASER E. (ESS-2)

VUATAZ, FRANCOIS (ESS-2)

21-6

LOGGING TECHNOLOGY FOR HIGH-

TEMPERATURE GEOTHERMAL BOREHOLES.

TIC.

1984. 10P. MN (LA-UR-84-1639) DENNIS, BERT R. (ESS-6)

21.7

LOW - TEMPERATURE GEOTHERMAL POTENTIAL OF THE OJO CALIENTE WARM SPRINGS AREA, NORTHERN NEW MEXICO.

LOS ALAMOS,

1984. 56P. (LA-10105-OBES)

VUATAZ, FRANCOIS (ESS-2)

STIX, JOHN (ESS-2)

GOFF, FRASER E. (ESS-2)

PEARSON, CHRISTOPHER F. (ESS-3)

21-8

PRELIMINARY REPORT ON FRACTURED IGNEOUS ROCK ENVIRONMENT TEST PITS.

TIC.

1983. 7P. MN (LA-UR-83-1797)

MATHEWS, MARK A. (ESS-3)

SCOTT, J.F.

LADELIFE, CAROL M. (ESS-3)
21-9

RESERVOIR SIZING USING INERT AND CHEMICALLYY REACTING TRACERS.

TIC.

1984. 25P. MN (LA-UR-84-2054)

ROBINSON, BRUCE A.

TESTER, JEFFERSON W.

BROWN, LEE F. (ESS-5)

21-10

ROOSEVELT HOT SPRINGS/HOT DRY ROCK PROSPECT AND EVALUATION OF THE ACCORD

1. 26 WELL.

तic.

1983. 4P. MN (LA-UR-83-1594)

SHANNON, SPENCER S., JR. (ESS-1)

ROWLEY, JOHN C. (ESS-4)

PETTITT, ROLAND A. (ESS-4)

GOFF, FRASER E. (ESS-2)

VUATAZ, FRANCOIS (ESS-2)

21-11

WELL LOG INTERPAETATION OF CERTAIN GEOTHERMAL FIELDS IN THE IMPERIAL

VALLEY, CALIFORNIA.

LOS ALAMOS,

1984. 23P. (LA-10067-MS)

ERSHAGHI, IRAJ

ABDASSAH, DODDY 


\section{GEOTHERMAL ENERGY - RESOURCE DEVELOPMENT}

22-1

DOES HYDRAULIC FRACTURING THEORY WORK IN JOINTED ROCK MASSES?

TIC,

1983. 7P. MN (LA-UR-83-1677)

MURPHY, HUGH D. (ESS-4)

KEPPLER, H. (ESS-4)

DASH, ZORA V. (ESS-4)

\section{2-2}

EVALUATION OF THE ST. LUCIA GEOTHERMAL RESOURCE. ENGINEERING INVESTIGATION

\section{AND COST ESTIMATE.}

LOS ALAMOS

1984. 51P. (LA-10209-MS)

ALTSEIMER, JOHN H. (S-4)

EDESKUTY, FREDERICK J. (S-4)

TAYLOR, WILLIAMB.

WILLIAMSON, KENNETH D., JR. (S-4

22-3

EVALUATION OF THE ST. LUCIA GEOTHERMAL RESOURCE - GEOLOGIC, GEOPHYSICAL AND HYDROGEOCHEMICAL INVESTIGATIONS.

LOS ALAMOS,

1984. 91P. (LA-10234-MS)

ANDER, MARK E. (ESS-3)

GOFF, FRASER E. (ESS-2)

HANOLD, ROBERT J. (ESS-DOT)

HEIKEN, GRANT H. (ESS-1)

VUATAZ, FRANCOIS (ESS-2)

WOHLETZ, KENNETH H. (ESS-1)
22-4

HDR GEOTHERMAL ENERGY - A PROGRESS REPORT.

TIC.

1983. 5P. MN (LA-UR-83-1569)

ABSTRACT PUBLISHED IN:

TRANS. AM. NUCL. SOC.,

V.45, P.6-7. 1983.

NUNZ, GREGORY J. (ESS-DOT)

FRANKE, PAUL R., JR. (ESS-DOT)

22-5

MICROEARTHQUAKES INDUCED DURING HYDRAULIC FRACTURING AT THE FENTON HILL HDR SITE - THE 1982 EXPERIMENTS.

TIC,

1983. 7P. MN (LA-UR-83-1678)

KEPPLER, H. (ESS-4)

PEARSON, CHRISTOPHER F. (ESS-3)

POTTER, ROBERT M. (ESS-4)

ALBRIGHT, JAMES N. (ESS-4)

22-6

PETROGRAPHIC ANALYSIS AND CORRELATION OF VOLCANIC ROCKS IN BOSTIC 1 - A WELL, NEAR MOUNTAIN HOME, IDAHO.

LOS ALAMOS,

1984. 29P. (LA-09966-HDR)

ARNEY, BARBARA H. (ESS-1)

GARDNER, JAMIE N. (ESS-2)

BELLUOMINI, STEPHEN G.
22-7

RECONNAISSANCE GEOLOGIC STRIP MAP FROM KINGMAN TO SOUTH OF BILL WILLIAMS MOUNTAIN, ARIZONA.

LOS ALAMOS.

1983. UNPAGED. (LA-09202-MAP)

GOFF, FRASER E. (ESS-2)

EDDY, ANDREA C. (G-6)

ARNEY, BARBARA H. (ESS-1)

22-8

USING CHEMICALLY REACTIVE TRACERS TO DETERMINE TEMPERATURE CHARACTERISTICS OF GEOTHERMAL RESERVOIRS.

TIC.

1984. 6P. MN (LA-UR-84-1186)

ROBINSON, BRUCEA.

TESTER, JEFFERSON W.

BROWN, LEE F. (ESS-5) 


\section{GENERAL REACTOR TECHNOLOGY}

24-1

BUCKLING OF STEEL CONTAINMENT SHELLS

UNDER TIME - DEPENDENT LOADING.

LOS ALAMOS.

1984. 33P. (LA-10087-MS)

BABCOCK, C. D., JR. (Q-13)

BAKER, WILFRED E.

FLY, GERALD W. (Q-13)

BENNETT, JOEL G. (Q-13)

$24-2$

CALIERATION AND QUALIFICATION OF THE

LOS ALAMOS FAILURE MODEL (LAFM).

LOS ALAMOS,

1984. 53P. (LA-10041-MS)

BAARS, RALPHE. (Q-7)

24-3

COMPARISON OF BEACON AND COMPARE REACTOR

CAVITY SUBCOMPARTMENT ANALYSES.

LOS ALAMOS.

1984. 51P. (LA-09776-MS)

BURKETT, MICHAEL W. (Q-6)

IDAR, E. S. (Q-6)

GIDO, RICHARD G. (Q-7)

LIME, JAMES F. (Q-7)

KOESTEL, A.

24-4

COMPUTER-AUTOMATED NEUTRON ACTIVATION ANALYSIS SYSTEM.

TIC.

1983. 7P. MN (LA-UR-83-3405)

MINOR, MICHAEL M. (INC-5)

GARCIA, SAMMY R. (INC-5)

24-5

EVALUATION OF THE CRACK PLANE

EQUILIBRIUM MODEL FOR PREDICTING

PLASTIC FRACTURE.

LOS ALAMOS,

1984. 17P. (LA-10129-MS)

BUTLER, THOMAS A. (Q-13)

SMITH, F. W.

24-6

FUEL ROD DEBRIS PACKING MODEL.

NUCL. TECHNOL,

V.67, P.66-71. 1984

MOORE, J. A.

24-7

HIGH-EFFICIENCY PARTICULATE AIR FILTER BEHAVIOR AT HIGH-SPEED FLOWS.

LOS ALAMOS,

1984. 12P. (LA-10078-MS)

TANG, P.K. (X-T)

GREGORY, WILLIAM S. (Q-6)

RICKETTS, C. I

SMITH, PHILLIP R.
24-8

HMS - A COMPUTER PROGRAM FOR TRANSIENT,

THREE - DIMENSIONAL MIXING GASES.

LOS ALAMOS

1984. 38P. (LA-10267-MS)

TRAVIS, JOHN R. (T-3)

24-9

LOS ALAMOS OMEGA WEST REACTOR.

TIC.

1983. 8P. MN (LA-UR-83-3404)

LYLE, ALVIN R. (INC-5)

WIUAMS, HERBERT T. (INC-5)

BUNKER, MERLE E. (INC-5)

24-10

LOW POWER UNATTENDED DEFENSE REACTOR.

TIC.

1984. 8P. MN (LA-UR-84-1480)

KIRCHNER, WALTER L. (Q-12)

MEIER, KARL L $(Q-13)$

24-11

NUCLEAR ELECTRIC CAPACITY EXPANSION IN

MEXICO - SYSTEM EFFECTS OF REACTOR

SIZE AND COST.

TRANS. AM. NUCL SOC.,

V.46, P.595-6. 1984.

ALSO PUBUSHED IN:

TIC.

1984. 5P. MN (LA-UR-84-0064)

THAYER, GARY R. (S-4)

ABBEY, DAVID S. (S-2)

HARDIE, R. WAYNE (S-DOT)

ENRIQUEZ, RAUL PEREZ

URIA, ERNESTO GRIJALVA

24-12

ON BROADENING FAILURE RATE DISTRIBUTIONS IN PRA UNCERTAINTY ANALYSES.

RISK ANAL.,

V.4, P.15-22. 1984.

MARTZ, HARRY F., JR. (S-1)

24-13

PROCEEDINGS OF NINTH ANNUAL STATISTICS SYMPOSIUM ON NATIONAL ENERGY ISSUES, OCTOBER 19-21, 1983

LOS ALAMOS,

1984. 195P. (LA-10127-C) BRYSON, MAURICE C. (S-1)

24-14

RADIATION TRANSPORT. OCTOBER 1, 1982

MARCH 31, 1983.

LOS ALAMOS

1984. 106P. (LA-10114-PR)

O'DELL, R. DOUGLAS $(X-6)$
24-15

REVIEW OF PROPOSED FAILURE CRITERIA

FOR DUCTILE MATERIALS.

LOS ALAMOS,

1984. 29P. (LA-10007-MS)

JU, F. D.

BUTLER, THOMAS A. (Q-13)

24-16

SOLA - PTS - A TRANSIENT, THREE-

DIMENSIONAL ALGORITHM FOR FLUID-

THERMAL MIXING AND WALL HEAT TRANSFER IN

COMPLEX GEOMETRIES.

LOS ALAMOS,

1984. 94P. (LA-10132-MS)

DALY, BART J. (T-3)

TORREY, MARTIN D. (T-3)

24-17

STATISTICAL ANALYSIS OF NUCLEAR POWER

PLANT PUMP FAILURE RATE VARIABIUY-

SOME PRELIMINARY RESULTS.

LOS ALAMOS

1984. 52P. (LA-10014-MS)

MARTZ, HARRY F., JR. (S-1)

WHITEMAN, DAVID E. (S-1)

24-18

THREE - DIMENSIONAL CALCULATIONS OF TRANSIENT FLUID - THERMAL MIXING IN THE DOWNCOMER OF THE CALVERT CUFFS - 1

PLANT USING SOLA - PTS.

LOS ALAMOS,

1984. 84P. (LA-10039-MS)

DALY, BART J. (T-3)

24-19

TRAC - PD2 INDEPENDENT ASSESSMENT.

LOS ALAMOS,

1984. 403P. (LA-10166-MS)

KNIGHT, THAD D. (Q-9)

24-20

TRAC-PF1. AN ADVANCED BEST-ESTIMATE COMPUTER PROGRAM FOR PRESSURIZED WATER REACTOR ANALYSIS.

LOS ALAMOS,

1984. 578P. (LA-09944-MS)

LLES, DENNIS R. (Q-9)

24-21

VERIFICATION OF MULTIDIMENSIONAL REACTOR

SAFETY ANALYSIS CODES DEVELOPED IN

GROUP T-3.

LOS ALAMOS,

1980. 26P. (LA-08637-MS)

DALY, BART J. (T-3) 


\section{GENERAL, MISCELLANEOUS, AND PROGRESS REPORTS (NONNUCLEAR)}

25-1

ACCOUNTING DIVISION - ORGANIZATIONAL

PROFILE.

LOS ALAMOS,

1984. 4P. (LALP-83-050)

SNYDER, CONNIE (IS-1)

25-2

ADMINISTRATIVE DATA PROCESSING

DIVISION - ORGANIZATIONAL PROFILE.

LOS ALAMOS,

1984. 4P. (LALP-83-060)

NEARY, JOAN (IS-1)

$25-3$

ALTERNATIVE ENERGY SOURCES PROGRAM

OFFICE - ORGANIZATIONAL PROFILE

LOS ALAMOS,

1984. 6P. (LALP-84-005)

NEARY, JOAN (IS-1)

25-4

ANALYSIS AND ASSESSMENT DIVISION-

ORGANIZATIONAL PROFILE.

LOS ALAMOS

1984. 4P. (LALP-84-032)

WALLER, RAY A. (S-DO)

25-5

ANNOTATED BIBLIOGRAPHY ON DECISION ANALYSIS WITH APPLICATION TO PROJECT

MANAGEMENT.

LOS ALAMOS,

1984. 49P. (LA-10027-MS)

BOOKER, JANE M. (S-1)

BRYSON, MAURICE C. (S-1)

25-6

APPLIED THEORETICAL PHYSICS DIVISIONORGANIZATIONAL PROFILE.

LOS ALAMOS,

1984. 6P. (LALP-83-061)

NEARY, JOAN (IS-1)

25-7

ATOMS FOR PEACE AFTER THIRTY YEARS

55P. CENTER FOR STRATEGIC AND

INTERNATIONAL STUDIES, 1984.

(333.792/A881PE)

BOLET, ADELA MARIA

EBINGER, CHARLES K.

PILAT, JOSEPH F. (ADPA-SA)

PENDLEY, ROBERT E. (ADPA)

25-8

BUDGET DIVISION - ORGANIZATIONAL

PROFILE

LOS ALAMOS,

1984. 2P. (LALP-83-054)

SNYDER, CONNIE (IS-1)
25-9

CENTER FOR MATERIALS SCIENCE-

ORGANIZATIONAL PROFILE.

LOS ALAMOS.

1984. 4P. (LALP-83-049)

SNYDER, CONNIE (IS-1)

25-10

EARTH AND SPACE SCIENCES DIVISION-

ORGANIZATIONAL PROFILE.

LOS ALAMOS,

1984. 5P. (LALP-84-048)

PONDER, GLENDA M. (ESS-4)

25-11

EVALUATION OF THE ST. LUCIA GEOTHERMAL

RESOURCE. MACROECONOMIC MODELS.

LOS ALAMOS,

1984. 89P. (LA-10212-MS)

BURRIS, ANTHONY E. (S-2)

TROCKI, LINDA K. (S-2)

YEAMANS, MARILYN K. (S-2)

KOLSTAD, CHARLES D.

25-12

HIGH TECHNOLOGY OPPORTUNITIES IN

NEW MEXICO - A PROFILE.

LOS ALAMOS,

1984. 123P. (LALP-84-017)

METROPOLIS, PAT H. (IS-1)

25-13

INSTITUTIONAL SUPPORTING RESEARCH

HIGHLIGHTS IN PHYSICS AND

MATHEMATICS, FISCAL YEAR 1983.

LOS ALAMOS

1984. 32P. (LALP-84-018)

VIGIL, JOHN C. (ADES)

25-14

ISOTOPES AND FUSION PROGRAM OFFICE-

ORGANIZATIONAL PROFILE

LOS ALAMOS

1984. 3P. (LALP-84-011)

NEARY, JOAN (IS-1)

25-15

LIFE SCIENCES DIVISION - ORGANIZATIONAL

PROFILE.

LOS ALAMOS,

1984. 8P. (LALP-83-024)

CHALLEM, JACK (IS-1)

25-16

MATERIALS MANAGEMENT DIVISION-

ORGANIZATIONAL PROFILE.

LOS ALAMOS.

1984. 4P. (LALP-83-053)

SNYDER, CONNIE (IS-1)
25-17

OBITUARY - HERBERT M. PARKER,

(1910-1984)

HEALTH PHYS

V.47,P.517-20. 1984.

HEALY, JOHN W. (HSE-DO)

25-18

OFFICE OF DEFENSE CONSTRUCTION-

ORGANIZATIONAL PROFILE.

LOS ALAMOS,

1984. 3P. (LALP-83-056)

NEARY, JOAN (IS-1)

25-19

PERFORMANCE APPRAISAL HANDBOOK

PERSONNEL APPRAISAL OUTLINE.

LOS ALAMOS,

1984. 11P. AND ATTACHMENTS AND

EXAMPLES. (LALP-84-019)

MC FARLAND, LARRY C. (ISD-6)

25-20

PUBLIC AFFAIRS OFFICE-ORGANIZATIONAL

PROFILE.

LOS ALAMOS,

1984. 4P. (LALP-84-024)

SNYDER, CONNIE (IS-1)

25-21

QUALITY CIRCLES IN A RESEARCH AND

DEVELOPMENT LABORATORY.

INTL. ASSOC. OF QUALITY CIRCLES, TRANSACTIONS, 6TH, 1984, CINCINNATI.

PROC. P.413-5. IACC. 1984.

CONNELLEE, BARBARA G. (MST-11)

THOMPSON, LOIS J. (PA-7)

25-22

RIO AZUL - MAYA CITY OF THE DEAD.

EXPLORERS $\mathrm{J}$.

V.62, P.162-7. 1984.

BARNETT, CHARLES R. (IS-2)

HARPISON, PETER D.

25-23

TECHNICAL AREA 55 ENTRY CONTROL SYSTEM (ECS).

TIC,

1984. 8P. MN (LA-UR-84-0419)

BEAUMONT, ARTHUR J. (MST-11)

BRUNDIGE, EDWARD L. (MST-11)

DESJARDIN, ROBERT (E-5)

RIVERA, RAYNALDO V. (E-5) 
25-24

TEST OPERATIONS OFFICE-

ORGANIZATIONAL PROFILE.

LOS ALAMOS,

1984. 4P. (LALP-83-059)

SNYDER, CONNIE (IS-1)

25-25

THOUGHTS ON HOW TO SOLVE THE

OVERCROWDING AT THE ANNUAL WEST COAST MEETING.

EOS,

V.65, P.98. 1984

BAKER, DANIEL N. (ESS-8)
25-26

U.S. STRATEGIC RESPONSES TO CONFLICT IN THE 1980S.

STRATEGIC RESPONSES TO CONFLICT IN

THE 1980S, W.J. TAYLOR, JR., S.A.

MAARANEN \& G.W. GONG, ED., P.483-99.

LEXINGTON BOOKS, 1984.

(355.033/S898RE)

TAYLOR, WILLIAM J., JR.

MAARANEN, STEVEN A. (ADPA)

GONG, GERRIT $W$.
25-27

WEAPONS PROGRAMS OFFICEORGANIZATIONAL PROFILE.

LOS ALAMOS,

1984. 3P. (LALP-84-010)

NEARY, JOAN (IS-1) 


\section{GENERAL, MISCELLANEOUS, AND PROGRESS REPORTS (NUCLEAR)}

26-1

ADVANCED WEAPONS TECHNOLOGY OFFICE

ORGANIZATIONAL PROFILE.

LOS ALAMOS

1984. 4P. (LALP-84-004)

SNYDER, CONNIE (IS-1)

26-2

APPENDIX.

NUCLEAR FISSION R\&D AND WASTE

MANAGEMENT, HEARINGS BEFORE THE ...

COMMITTEE ON SCIENCE \& TECHNOLOGY

U.S. HOUSE ... 98TH CONGRESS ...

1983, WASHINGTON. PROC.. P.721-33.

GPO, 1984. (328/C98-1/83-20/N.4)

HANSON, JOHN E. (Q-DO)

263

CONDITIONS AFFECTING THE BACKFIL IN A

WASTE PACKAGE FOR A REPOSITORY IN TUFF. NUCLEAR WASTE MANAGEMENT PROGRAM, SUMMARY REPORT, R.M. CHUNG, ED.,

P.57-8. NBS, 1983.

(621.483/W92BRE/1981)

JOHNSTONE, J. KEITH

VINE, ELIZABETHN. (CNC-7)

26-4

DIRECTED ENERGY BEAM WEAPONS

ELECTRO-CULTURE, 1984, ARLINGTON.

PROC., P.79-86. SPIE, 1984.

(620/E38CU/1984)

BOYER, KEITH (DIR-0)

26-5

ELECTRON COLLISION PROCESSES IN THE

EARTH'S ATMOSPHERE.

UNITED STATES-JAPAN SEMINAR ON

ELECTRON-MOLECULE COLLISIONS AND

PHOTOIONIZATION PROCESSES, 1ST, 1982,

PASADENA. PROC., P.145-51. VERLAG

CHEMIE INTL., 1983.

(539/U58EL/1ST/1982)

CARTWRIGHT, DAVID C. (NSP/IF)

26-6

ENGINEERING SCIENCES RESEARCH

HIGHLIGHTS FISCAL YEAR 1983

LOS ALAMOS,

1984. 42P. (LALP-84-014)

TUCKER, EVA F. $(W X-4)$

DOBRATZ, BRIGITTA $(M-1)$

26-7

IMPLICATIONS OF REDUCED NATO NUCLEAR

STOCKPILES.

LOS ALAMOS,

1984:35P. (LA-10221-MS)

SANDOVAL, RICHARD R. (S-6)
26-8

LASER-BASED DATA ACQUISITION IN GAS CENTRIFUGE ENVIRONMENTS USING OPTICAL FIBERS.

FIBER OPTICS IN ADVERSE ENVIRONMENTS, 2ND, 1984, SAN DIEGO. PROC., P.64-8. SPIE, 1984. (621.369/F443OP/1984)

CATES, MICHAEL REID

ALLISON, S. W.

MARSHALL, B.R.

DAVIES, T.J.

FRANKS, L A

NELSON, M. A.

NOEL, BRUCE W. $(\mathrm{WX}-10)$

26-9

LINEAR ALGEBRAIC APPROACH TO

ELECTRON-MOLECULE COLLISIONS. UNITED STATES-JAPAN SEMINAR ON ELECTRON-MOLECULE COLLISIONS AND PHOTOIONIZATION PROCESSES, 1ST, 1982. PASADENA. PROC., P.203-10. VERLAG CHEMIE INTL., 1983.

(539/U58EL/1ST/1982)

SCHNEIDER, BARRY I. (T-12)

COLLINS, LEE A. $(T-4)$

26-10

LOS ALAMOS NATIONAL LABORATORY'S

EDUCATIONAL OUTREACH PROGRAM

TRANS. AM. NUCL SOC.

V.46, P.30-2. 1984

SANDOVAL, ABADELI (IS-DO)

BERG, DONNA (IS-4)

26-11

PREPARED STATEMENT OF JOHN E HANSON, PROGRAM MANAGER, SPACE AND MILITARY REACTOR PROGRAM, LOS ALAMOS NATIONAL LABORATORY.

NUCLEAR FISSION R\&D AND WASTE

MANAGEMENT, HEARINGS BEFORE THE ... COMMITTEE ON SCIENCE \& TECHNOLOGY

U.S. HOUSE ... 98TH CONGRESS ... 1983, WASHINGTON. PROC., P.708-17.

GPO, 1984. (328/C98-1/83-20/V.4) HANSON, JOHN E. (Q-DO)
26-12

PUBLICATIONS OF LOS ALAMOS RESEARCH 1983.

LOS ALAMOS,

1984. 182P. (LA-10155-MS)

SHERIDAN, CONNIE J. (IS-4)

MC CLARY, WILMA J. (IS-4)

RICH, JEANETTE A. (IS-4)

RODRIGUEZ, LA VERNE L. (IS-4)

26-13

RARE GAS HALOGEN EXCIMERS.

EXCIMER LASERS, CH.K. RHODES, ED., P.87-137. SPRINGER-VERLAG, 1984.

(621.366/E96LA/1984)

BRAU, CHARLES A. (AT-DO)

26-14

REMARKS OF MICHAEL E. BERGER, DEPUTY ASSOCIATE DIRECTOR, LOS ALAMOS NATIONAL LABORATORY BEFORE THE SUBCOMMITTEE ON ENERGY DEVELOPMENT AND APPLICATIONS AND ENERGY RESEARCH AND PRODUCTION OF THE HOUSE SCIENCE AND TECHNOLOGY COMMITTEE. INTEGRITY OF ELECTRIC POWER SYSTEMS HEARING BEFORE THE ... U.S. HOUSE, ... 97TH CONGRESS ..., 1982, WASHINGTON. PROC., P 21-30. GPO 1983. (328/C97-2/82-79)

BERGER, MICHAEL E. (EP/AES)

26-15

RESPONSES TO QUESTIONS FROM THE HOUSE SCIENCE \& TECHNOLOGY COMMITTEE FOLLOWING TESTIMONY OF MICHAEL E. BERGER BEFORE SUBCOMMITTEE ON ENERGY DEVELOPMENT AND APPLICATIONS AND SUBCOMMITTEE ON ENERGY RESEARCH AND PRODUCTION US HOUSE OF

REPRESENTATIVES DECEMBER 9, 1982. INTEGRITY OF ELECTRIC POWER SYSTEMS HEARING BEFORE THE ... U.S. HOUSE, ... 97TH CONGRESS ..., 1982, WASHINGTON. PROC., P.113-21. GPO, 1983. (328/C97-2/82-79)

BERGER, MCHAEL E. (EP/AES)

26-16

STATEMENT OF DR. ROBERT N. THORN LOS

ALAMOS NATIONAL LABORATORY.

NATIONAL DEFENSE PROGRAMS

AUTHORIZATION HEARING BEFORE THE. COMMITTEE ON ARMED SERVICES U.S. SENATE ... 98TH CONGRESS ... 1983. WASHINGTON. PROC., P.80, 91 3. GPO, 1983. (328/C98-1/83-75)

THORN, ROBERT N. (DIR-O) 
26-17

STATEMENT OF JIMMY MCCLARY, DIVISION LEADER FOR OPERATIONAL SECURITY AND SAFEGUARDS DIVISION, LOS ALAMOS NATIONAL LABORATORY, LOS ALAMOS, N. MEX. ACCOMPANIED BY DOTTY CAMILLO, GROUP LEADER, COMMUNICATIONS AND TELECOMMUNICATIONS GROUP, LOS ALAMOS NATIONAL LABORATORY, NEW MEXICO.

COMPUTER AND COMMUNICATIONS SECURITY AND PRIVACY, HEARINGS BEFORE THE ...

U.S. HOUSE ... 98TH CONGRESS ...

1983, WASHINGTON. PROC., P.34-53.

GPO, 1984. (328/C98-1/83-128)

MCCLARY, JIMMY F. (IT-3)

CAMILLO, DOROTHY E. (OS-4)
26-18

STATEMENT OF JOHN E. HANSON, PROGRAM MANAGER FOR SPACE AND MILITARY REACTOF PROGRAMS, LOS ALAMOS NATIONAL

LABORATORY. NUCLEAR FISSION RED AND WASTE MANAGEMENT, HEARINGS BEFORE THE ... COMMITTEE ON SCIENCE \& TECHNOLOGY U.S. HOUSE ... 98TH CONGRESS ... 1983, WASHINGTON. PROC., P.705-7. GPO, 1984. (328/C98-1/83-20/N.4) HANSON, JOHN E. (Q-DO)

26-19

STATEMENTS OF ... DR. JAMES M. WILLIAMS, ASSISTANT DIRECTOR FOR PLANNING AND ANALYSIS, LOS ALAMOS NATIONAL LABORATORY, LOS ALAMOS, N. MEX.; ... JOINT INDUSTRY/UNIVERSITY COOPERATION WITH FEDERALLY SUPPORTED RESEARCH FACILITIES, HEARING BEFORE THE ...

U.S. HOUSE ... 98TH CONGRESS, ... 1983, WASHINGTON. PROC., P.17-30. GPO, 1983. (328/C98-1/83-43) WILLIAMS, JAMES M. (ADPA)
26-20

TOKAMAK REACTOR STARTUP POWER. SYMPOSIUM ON FUSION ENGINEERING, 10TH, 1983, PHILADELPHIA. PROC., ORNL, 1983.

(621.484/S989FUA/10TH/1983) WELDON, DAVID M. (CTR-9) MURRAY, JOHN G.

26-21

TYPICAL LOS ALAMOS NATIONAL LABORATORY UNDERGROUND NUCLEAR TEST.

LOS ALAMOS

1984. 5P. (LALP-84-047)

WOLFF, WALTER P. (NSP/TO) 



\section{HEALTH AND SAFETY}

28-1

ACCEPTANCE - TESTING PROCEDURES FOR

AIR - LINE SUPPLIED - AIR SUITS.

LOS ALAMOS.

1984. 23P. (LA-10156-MS)

BRADLEY, ORVIL D. (HSE-5)

28-2

APPARATUS FOR MONITORING TRITIUM IN TRITIUM CONTAMINATING ENVIRONMENTS USING A MODIFIED KANNE CHAMBER

PATENTED

APR. 24, 1984, FILED 1981.

(U.S. PATENT 4,445.037)

ANDERSON, DAVID F. (H-1)

28-3

BRAIN TUMORS AT A NUCLEAR FACILITY.

J. OCCUP. MED.,

V.26, P.721-4. 1984.

REYES, MICHELE (HSE-14)

WILKINSON, GREGG S. (HSE-14)

TIETJEN, GARYL. (S-1)

VOELZ, GEORGE L. (HSE-DO)

ACQUAVELLA, JOHN F.

BISTLINE, ROBERT

28-4

CHEMICAL PERMEATION - A SUMMARY REPORT OF RECENT NIOSH-DIRECTED STUDIES AT THE

LOS ALAMOS NATIONAL LABORATORY.

AM. IND. HYG. ASSOC. J.

V.45, P.B10-2. 1984

STAMPFER, JOSEPH F., JR. (HSE-5)

MC LEOD, MIA J. (HSE-5)

BETTS, M. R. (HSE-5)

MARTINEZ, AARON M. (HSE-5)

BERARDINELLI, S.P.

28-5

COMPARATIVE STUDY OF HEPA FILTER

EFFICIENCIES WHEN CHALLENGED WITH

THERMAL-AND AIR-JET-GENERATED

DI-2-ETHYLHEXYL SEBECATE,

DI-2-ETHYLHEXYL PHTHALATE, AND SODIUM

CHLORIDE.

LOS ALAMOS.

1984. 64P. (LA-09985-MS)

KERSCHNER, HARRISON F. III

ETTINGER, HARRY J. (HSE-5)

DE FIELD, JAMES D. (HSE-5)

BECKMAN, RICHARD J. (S-1)

28-6

COMPARISON OF TWO LUNG CLEARANCE MODELS

BASED ON THE DISSOLUTION RATES OF

OXIDIZED DEPLETED URANIUM.

LOS ALAMOS,

1984. 67P. (LA-10214-T)

CRIST, KEVIN C.
28-7.

COMPUTER BASED PLUTONIUM WOUND

MONITOR.

IEEE TRANS. NUCL. SCI.

V.NS-31, P.679-80. 1984.

WAECHTER, DAVID A. (E-7)

BRAKE, RICHARD JOSEPH (H-4)

VASILIK, DENNIS G. (H-4)

ERKKILA, BRUCE H. $(\mathrm{H}-4)$

28-8

COMPUTERIZED PLUTONIUM WOUND ANALYSIS SYSTEM.

TIC.

1983. 5P. MN (LA-UR-83-2958)

WAECHTER, DAVID A. (E-1)

BRAKE, RICHARD JOSEPH (H-4)

VASILIK, DENNIS G. (HSE-1)

ERKKILA, BRUCE H. (IT-1)

28-9

ENVIRONMENTAL SURVEILLANCE AT LOS

ALAMOS DURING 1983

LOS ALAMOS,

1984. 180P. (LA-10100-ENV)

ENVIRONMENTAL SURVEILLANCE GROUP (H-8)

28-10

ESTIMATING THE RISKS OF CANCER MORTALITY

AND GENETIC DEFECTS RESULTING FROM

EXPOSURES TO LOW LEVELS OF IONIZING

RADIATION.

LOS ALAMOS.

1984. 113P. (LA-09893-MS)

BUHL, THOMAS E. (H-8)

HANSEN, WAYNE R. (H-8)

28-11

EVALUATION OF AEROSOL SPECTROMETERS FOR QUALITY ASSURANCE FILTER PENETRATION

MEASUREMENTS.

AEROSOLS CONFERENCE, 1984

MINNEAPOLIS, PROC., P.547-50.

ELSEVIER, 1984.

SCRIPSICK, RONALD C. (HSE-5)

SODERHOLM, SIDNEY C. (HSE-5)

TILLERY, MARVIN I. (HSE-5)

28-12

EVAL̈UATION OF SPURIOUS READINGS IN

LOS ALAMOS PERSONNEL TL DOSIMETERS.

LOS ALAMOS,

1984, 13P. (LA-10140-MS)

EISEN, YOSEF Y. (HSE-1)

LITTLEJOHN, GEORGE J. (HSE-1)

CORTEZ, JOSEPH R. (HSE-1)
28-13

GENOTOXICITY OF ALPHA PARTICLES IN

HUMAN EMBRYONIC SKIN FIBROBLASTS.

RADIAT. RES.

V.100, P.321-6. 1984

CHEN, DAVID JEN-CHI (LS-3)

STRNISTE, GARY F. (LS-3)

TOKITA, NOBUHIKO (LS-1)

28-14

GUIDELINES FOR THE SELECTION OF CHEMICAL PROTECTIVE CLOTHING.

GUIDELINES FOR THE SELECTION OF CHEMICAL PROTECTIVE CLOTHING,

ARTHUR D. LTTLE, INC. 76P.

1983 (620.86/G946SEN.1)

SCHWOPE, ARTHUR D.

COSTAS, P. P.

JACKSON, JAMES O. (HSE-5)

WEITZMAN, D. J.

28-15

GUIDELINES FOR THE SELECTION OF CHEMICAL PROTECTIVE CLOTHING

GUIDEUNES FOR THE SELECTION OF CHEMICAL PROTECTIVE CLOTHING,

ARTHUR D. LITTLE, INC., 41P.

1983. (620.86/G946SE/N.2)

SCHWOPE, ARTHUR D.

COSTAS, P. P.

JACKSON, JAMES O. (HSE-5)

WEITZMAN, D. J.

28-16

HUMTRN - DOCUMENTATION AND VERIFICATION FOR AN ICRP-BASED AGE-AND SEX-SPECIFIC HUMAN SIMULATION MODEL FOR RADIONUCUDE DOSE ASSESSMENT.

LOS ALAMOS,

1984. 83P. (LA-09994-MS)

GALLEGOS, ANTHONY F. (H-B)

WENZEL, WALTER J. (HSE-8)

28-17

IMAGING RADIATION DETECTOR WITH GAIN.

PATENTED

OCT. 23, 1984, FILED 1982

(U.S. PATENT-4,479.059)

MORRIS, CHRISTOPHER L. (MP-10)

IDZOREK, G. C. (MP-10)

ATENCIO, L. G. (MP-10)

28-18

IN - PLACE TESTING OF MULTIPLE STAGE

FILTER SYSTEMS WITHOUT DISRUPTION OF

PLANT OPERATIONS IN THE PLUTONIUM

FACILITY AT LOS ALAMOS.

TIC,

1984. 12P. MN (LA-UR-84-2319)

ORTIZ, JOHN P. (HSE-5) 
26-19

IN VIVO ASSESSMENT OF LUNG BURDENS AT

THE LOS ALAMOS NATIONAL LABORATORY.

LOSALAMOS,

1984. 25P. (LA-09978-MS)

VASILIK, DENNIS G. (HSE-1)

AIKIN, IRENE C. (HSE-1)

COOP, K. L. (Q-2)

UMBARGER, C. JOHN (IT-1)

\section{8-20}

LITERATURE REVIEW ON AEROSOL SAMPLING

DEVICES FOR RESPIRATORY FIELD STUDIES.

LOS ALAMOS

1984. 56P. (LA-09977-MS)

SUTCLIFFE, CAROL R. (HSE-5)

28-21

OCCUPATIONAL HEALTH AND ENVIRONMENTAL RESEARCH PROGRAM OF THE HEALTH DIVSION. 1982.

LOS ALAMOS,

1984. 110P. (LA-09958-PR)

VOELZ, GEORGE L. (HSE-DO)

\section{8-22}

PASSIVE DOSIMETERS FOR RF AND

MICROWAVE FIELDS.

REV.SCI. INSTRUM.;

V.55, P.424-6. 1984

DOSS. JAMES D. (MP-3)
28-23

PLUTONIUM PARTITIONING AMONG INTERNAL ORGANS.

HEALTH PHYS.,

V.46, P.839-43. 1984

THOMAS, ROBERT G. (H-DO)

HEALY, JOHNW. (H-DO)

MC INROY, JAMES F. (H-5)

28-24

PORTABLE BETA SPECTROMETER - DOSIMETER.

TIC.

1983. 6P. MN (LA-UR-83-2959)

ERKKILA, BRUCEH. (IT-1)

WAECHTER, DAVID A. (E-1)

BRAKE, RICHARD JOSEPH (H-4)

28-25

QUALTY CONTROL ACTIVITIES IN SUPPORT OF THE PLUTONIUM WORKERS STUDY.

ASSESSMENT OF CODING CONSISTENCY FOR DATA COLLECTED AT ROCKY FLATS.

LOS ALAMOS,

1984. 8P. (LA-09462-MS)

REYES, MICHELE (H-14)

WILKINSON, GREGG S. (H-14)

ACQUAVELLA, JOHN F. (HSE-14)
28-26

QUALTY CONTROL PROCEDURES FOR

COMPUTERIZING EMPLOYEE DATA FOR A STUDY

OF MOARTALTY AMONG WORKERS AT THE

PANTEX PLANT.

LOS ALAMOS

1984. BP. (LA-10279-MS)

WIGGS, LAURIE D. (HSE-14)

MASTERSON. JEANNENE M. (M-4)

ACQUAVELLA, JOHN F.

28-27

THERMALIY STIMULATED LUMINESCENCE IN

BIOCHEMICAL SYSTEMS.

RADIAT. PROT. DOSIM.

V.8, P.117-38. 1984

COOKE, D. W. (MP-3)

28-28

URANIUM INTERNAL EXPOSURE EVALUATION

BASED ON URINE ASSAY DATA.

LOS ALAMOS,

1984. 24P. (LA-10246-MS)

LAWRENCE, JAMES N. P. (H-1) 


\section{HEATING AND COOLING-RESIDENTIAL AND COMMERCIAL APPLICATIONS}

29-1

ANALYSIS OF VALIDATION DATA SETS IN THE

CLASS A PERFORMANCE VALUATION PROGRAM.

TIC,

1983. 7P. MN (LA-UR-83-1968)

HUNN, BRUCE D. (Q-11)

29-2

CONSERVATION AND SOLAR GUIDELINES.

TIC.

1983. 7P. MN (LA-UR-83-1871)

BALCOMB, J. DOUGLAS (Q-DO)

29-3

CONVECTIVE HEAT TRANSFER INSIDE PASSIVE SOLAR BUILDINGS.

TIC.

1983. 8P. MN LA-UR-83-2545)

JONES, ROBERT W. (Q-11)

BALCOMB, J. DOUGLAS (Q-DO)

YAMAGUCHI, KENJIRO

29-4

COOLING - LOAD IMPLICATIONS FOR

RESIDENTIAL PASSIVE SOLAR HEATING SYSTEMS.

TIC

1983. 8P. MN (LA-UR-83-2647)

JONES, ROBERT W. (Q-11)

MC FARLAND, ROBERT D. (Q-11)

29-5

HEAT DISTRIBUTION BY NATURAL

CONVECTION

TIC,

1983. 7P. MN (LA-UR-83-1872)

BALCOMB, J. DOUGLAS (Q-DO)

YAMAGUCHI, KENJIRO

29-6

INCLUDING THE EFFECT OF CONTROI

STRATEGY IN SOLAR LOAD RATIO

CALCULATIONS.

TIC,

1984. 6P. MN (LA-UR-84-1338)

WRAY, WILLIAM O. (Q-13)

KOSIEWICZ, CLAUDIAE. (Q-13)
29-7

MASS AND FANS IN ATTACHED SUNSPACES

ENERGY CONSERVING GREENHOUSES, 3RD,

1982, HYANNIS. PROC., P.81-90.

AMERICAN SOLAR ENERGY SOCIETY,

1983. (690.892/E56CO/1982)

ALSO PUBLISHED IN:

TIC,

1982. 11P. MN (LA-UR-83-0199)

JONES, ROBERT W. (Q-11)

MC FARLAND, ROBERT D. (Q-11)

LAZARUS, GLORIA S. (Q-11)

29-8

MATERIALS ASPECTS OF SOLAR ENERGY USE IN BUILDINGS.

TIC.

1984. 9P. MN (LA-UR-84-1428)

MOORE, STANLEY W. (Q-13)

MC FARLAND, ROBERT D. (Q-11)

29-9

PASSIVE AND LOW ENERGY RESEARCH AND DEVELOPMENT - A GLOBAL VIEW.

TIC,

1984. 11P. MN (LA-UR-84-1507)

BALCOMB, J. DOUGLAS (Q-13)

29-10

PASSIVE SOLAR HEATING ANALYSIS. A NEW ASHRAE MANUAL.

TIC,

1983. 10P. MN (LA-UR-83-1209)

BALCOMB, J. DOUGLAS (Q-DO)

29-11

PASSIVE SOLAR HEATING AND ANALYSIS

TIC,

1984. 21P. MN (LA-UR-84-1294)

JONES, ROBERT W. (Q-11)

29-12

PASSIVE SOLAR RESEARCH AND PRACTICE.

ENERGY BUILD.,

V.7, P.281-95. 1984.

ALSO PUBLISHED IN:

TIC,

1983. 17P. MN (LA-UR-83-0990)

BALCOMB, J. DOUGLAS (Q-DO)
29-13

PREDICTION OF INTERNAL TEMPERATURE SWINGS IN DIRECT GAIN PASSIVE SOLAR BUILDINGS

TIC.

1983. 7P. MN (LA-UR-83-2246) BALCOMB, J. DOUGLAS (O-DO)

29-14

PROGRESS ON SOLAR ABSORBER SELECTIVE PAINT RESEARCH.

OPTICAL MATERIALS TECHNOLOGY FOR ENERGY EFFICIENCY AND SOLAR ENERGY CONVERSION III, 1984, SAN DIEGO.

PROC., P.68-76. SPIE, 1984.

(621.47/062MA/1984)

MOORE, STANLEY W. (Q-13)

29-15

SLR METHODS FOR ATTACHED SUNSPACES. ENERGY CONSERVING GREENHOUSES, 3RD, 1982, HYANNIS, PROC., P.163-72.

AMERICAN SOLAR ENERGY SOCIETY, 1983. (690.892/E56CO/1982)

ALSO PUBLISHED IN:

TIC

1982. 11P. MN (LA-UR-82-3634)

JONES, ROBERT W. (Q-11)

MC FARLAND, ROBERT D. (Q-11)

29-16

STUOY OF THERMOSIPHON AND RADIANT

PANEL PASSIVE HEATING SYSTEMS FOR

METAL BUILDINGS.

TIC.

1983. 5P. MN (LA-UR-83-1967)

BIEHL, FRANZ A. (Q-11)

SCHNURR, NORMAN M. (WX-4)

WRAY, WILLIAM O. (Q-11)

29-17

VAPOR - PHASE HEAT - TRANSPORT SYSTEM.

TIC.

1983. 7P. MN (LA-UR-83-2646)

HEDSTROM, JAMES C. (Q-11) 
30-1

"FIRST STEP" BLANKET STRUCTURE AND FUEL

ASSEMBLY DESIGN.

TRANS. AM. NUCL. SOC.,

V.46, P.242-3. 1984.

ALSO PUBLISHED IN:

TIC,

1984. 6P. MN (LA-UR-84-0169)

SAYLOR, WILLIAM W. (S-4)

PENDERGRASS, JOHN H. (S-4)

THAYER, GARY R. (S-4)

30-2

"FIRST STEP" TOWARD ICF

COMMERCIALIZATION.

TRANS. AM. NUCL. SOC.,

V.46, P.241-2. 1984.

ALSO PUBLISHED IN:

TIC,

1984. 6P. MN (LA-UR-84-0168)

SAYLOR, WILLIAM W. (S-4)

PENDERGRASS, JOHN H. (S-4)

DUDZIAK, DONALD J. (S-4)

$30-3$

AEM/STEM ANALYSIS OF VAPOR DEPOSITED

MULTILAYERED LASER TARGETS.

MICROBEAM ANALYSIS SOCIETY CONFERENCE

18TH, 1983, PHOENIX. PROC., P.171-4.

SAN FRANCISCO PRESS, 1983.

ALSO PUBLISHED IN:

TIC,

1983. 16P. MN (LA-UR-B3-0819)

JOHNSON, KAYE ALLAN (MST-11)

STAUDHAMMER, KARL P. (MST-5)

REEVES, GARY ALAN (MST-6)

VEESER, LYNN R. (P-14)

$30-4$

APPLICATION FOR SURVEYING TECHNOLOGY FOR

THE ALIGNMENT OF LARGE OPTICAL SYSTEMS.

TIC,

1984. 11P. MN (LA-UR-84-0034)

BAUKE, WALTER (P-5)

30-5

CONVECTIVE MECHANISM FOR INHIBITION

OF HEAT CONDUCTION IN LASER PRODUCED

PLASMAS.

TIC,

1984. 7P. MN (LA-UR-84-1154)

LEE, PETER H. Y. (P-1)

WILLI, OSWALD (P-1)

TRAINOR, ROBERT JAMES (P-1)

30-6

CONVECTIVE TRANSPORT IN LASER TARGET

PLASMAS.

TIC,

1982. 17P. MN (LA-UR-83-0586)

BRACKBILL, JEREMIAH U. $(X-1)$

COLOMBANT, D.

GRANDJOUAN, $N$.
30-7

DETERMINATION OF LASER INTENSITY AND

HOT-ELECTRON TEMPERATURE FROM FASTEST

ION VELOCITY MEASUREMENT ON LASER-

PRODUCED PLASMA.

PHYS. FLUIDS,

V.27, P.296-301. 1984.

TAN, TAI HO (P-4)

MC CALL, GENE H. (X-DO)

WILLIAMS, ARTHUR H. (P-4)

30-8

DEVELOPMENT OF CARBON DIOXIDE AND KRYPTON FLUORINE GAS LASERS AS DRIVERS FOR INERTIAL CONFINEMENT FUSION.

TIC,

1983. 16P. MN (LA-UR-83-3470)

ROCKWOOD, STEPHEN D. (NSP/IF)

30-9

EVIDENCE FOR A HOT ELECTRON FLUX LMIT

IN LASER - PRODUCED PLASMA EXPERIMENTS.

TIC,

1983. 11P. MN (LA-UR-83-2568)

MC CALL, GENE H. (X-DO)

$30-10$

EXCIMER LASERS FOR INERTIAL CONFINEMENT

FUSION.

TRANS. AM. NUCL. SOC.

V.46, P.187-8. 1984.

SINGER, SIDNEY (P-DO)

30-11

HEAVYION FUSION PHYSICS ISSUES

TIC,

1984. 13P. MN (LA-UR-84-0606)

BANGERTER, ROGER O. (NSP/IF)

30-12

INERTIAL FUSION COMMERCIAL-

APPLICATIONS DRIVER COST ISSUES AND

(SOME) ANSWERS.

TRANS. AM. NUCL. SOC.,

V.46, P.192-4. 1984.

PENDERGRASS, JOHN H. (S-4)

30-13

LASER - PLASMA INTERACTION AT 10.6

MICRONS.

TIC.

1983. 36P. MN (LA-UR-83-2840)

JONES, ROGER D. $(x-1)$

KINDEL, JOSEPH M. $(X-1)$
30-14

LASER DAMAGE RESULTS AND ANALYSES FOR ULTRAVIOLET REFLECTORS UNDER MULTIPLESHOT IRRADIATION.

LASER INDUCED DAMAGE IN OPTICAL

MATERIALS, 13TH, 1981, BOULDER.

PROC., P.350-62. NBS, 1983.

(620.112/S989OM/13TH/1981)

FOLTYN, STEPHEN R. (AP-2)

NEWNAM, BRIAN E. (AP-2)

JOLIN, L. J. (AP-2)

30-15

MEASUREMENT AND ANALYSIS OF NEAR-

CLASSICAL THERMAL TRANSPORT IN ONE-

MICRON LASER-IRRADIATED SPHERICAL

PLASMAS.

PHYS. REV. LETT.

V.53, P.2563-6. 1984

HAUER, ALLAN (P-4)

MEAD, W. C. $(X-1)$

WILLI, OSWALD (P-1)

KILKENNY, J.D.

BRADLEY, DAVID

TABATABAEI, S. D.

HOOKER, C.

30-16

OPERATIONAL PERFORMANCE OF THE ANTARES

ALIGNMENT SYSTEM

OPTICAL ALIGNMENT II, 1984.

ARUNGTON. PROC., P.113-9. SPIE.

1984. (621.36/O62A/1984)

BENDER, STEVEN C. (P-5)

APPERT, QUENTIN D. (P-5)

30-17

OPTICAL TOOLING FOR THE FUNCTIONAL

ALIGNMENT OF TILTED AND DECENTERED

OPTICAL TRAINS.

OPTICAL ALIGNMENT II, 1984,

ARLINGTON. PROC., P.104-12. SPIE,

1984. (621.36/O62A/1984)

BAUKE, WALTER (P-5)

CROSS, EUGENE W. (P-5)

30-18

PLASMA SIMULATION STUDIES OF ELECTRON

ACCELERATION FOR BEAT WAVE AND SINGLE FREQUENCY - LASER HEATING SCHEMES.

TIC,

1984. 5P. MN (LA-UR-84-1063)

KINDEL, JOSEPH.M. $(X-1)$

FORSLUND, DAVID $W .(\alpha-1)$

MORI, WARREN $\mathrm{B} .(\mathbf{X}-1)$

JOSHI, C

DAWSON, J.M. 
30-19

SUPRATHERMAL ELECTRON GENERATION TRANSPORT AND DEPOSITION IN CARBON DIOXIDE LASER IRRADIATED TARGETS.

LASER INTERACTION AND RELATED

PLASMA PHENOMENA, H. HORA AND

G.H. MILEY, EDS., P.479-91. PLENUM,

1984. (535/L343IN/N.6)

HAUER, ALLAN (P-4)

GOLDMAN, ROBERT S. (X-1)

KRISTAL, RICHARD (P-4)

YATES, MARY ANNE (P-4)

MUELLER, $M$.

BEGAY, FRED (P-4)

VAN HULSTEYN, DAVID B. (P-14)

MITCHELL, KENNETH B. (P-4)

KEPHART, JOHN F. (P-4)

OONA, HENN (P-7)

STOVER, ELMER K. $(X-1)$

BRACKBILL, JEREMIAH U. $(X-1)$

FORSLUND, DAVID W. $(X-1)$
30-20

TRITIUM AND PLUTONIUM PRODUCTION AS A

STEP TOWARD ICF COMMERCIALIZATION.

TIC.

1983. 6P. MN (LA-UR-83-3229)

PENDERGRASS, JOHN H. (S-4)

DUDZIAK, DONALD J. (S-4) 


\section{INSTRUMENTS}

31-1

APPARATUS AND METHOD FOR DOWNHOLE INJECTION OF RADIOACTIVE TRACER.

\section{PATENTED}

DEC. 20, 1983, FILED 1980

(U.S.PATENT 4,421,982)

POTTER, ROBERT M. (ESS-4)

ARCHULETA, JACOBOR. (G-4)

FINK, CONRAD F. (G-4)

31-2

APPARATUS FOR RECOVERING GASEOUS

HYDROCARBONS FROM HYDROCARBON-

CONTAINING SOLID HYDRATES.

PATENTED

JAN. 10, 1884, FILED 1982.

(U.S.PATENT-4,424,858)

ELLIOT, GUY R. B. (G-9)

BARRACLOUGH, BRUCEL (CMB-1)

VANDERBORGH, NICHOLASE. (E-11)

$31-3$

AUTOMATIO VOLTAGE IMBALANCE

DETECTOR.

PATENTED

JAN. 3, 1984, FILED 1981.

(U.S.PATENT-4,424,491)

BOBBETT, RONALD E. (E-11)

MC CORMICK, J. BYRON (E-DO)

KERWIN, WILLAM J.

$31-4$

CHANNEL MULTIPLIER COMPATIBLE MATERIALS AND LIFETIME TESTS.

REV. SCI. INSTRUM.

V.55, P.463-7. 1984.

MC COMAS, DAVID J. (ESS-8)

BAME, SAMUEL J., JR. (ESS-8)

31.5

CHARACTERIZATION OF A FAST, SENSITIVE

GAMMA DETECTOR UTILIZING MICROCHANNEL

PLATE PHOTOMULTIPLIER.

IEEE TRANS. NUCL. SCI.

V.NS-31, P.387-9. 1984.

KRUSE, HERALD W. (P-14)

HOCKER LP.

$31-6$

CHOOSING AN IMAGING SYSTEM.

TIC.

1983. 5P. MN (LA-UR-83-2312)

ABSTRACT PUBLISHED IN:

TRANS. AM. NUCL. SOC.,

V.45, P.252-3. 1983.

FENIMORE, EDWARD E. (ESS-9)
$31-7$

CONCEPTUAL DESIGN AND ANALYSIS OF

HIGH SPEED ELECTRONIC IMAGING.

LOS ALAMOS,

1984. 47P. (LA-10072-MS)

BROWN, R. R. (E-8)

PARKER, JOSEPH R. (P-5)

31-8

DETERMINATION OF THE ABSOLUTE EFFICIENCY

OF AN ORGANIC SCINTILLATOR FOR NEUTRONS WITH ENERGIES BETWEEN 0.5 AND 800 MEV.

NUCL. INSTRUM. METHODS PHYS. RES.,

V.227, P.565-70. 1984

HOWE, S. D. (X-2)

LISOWSKI, PAUL W. (P-3)

RUSSELL, QARY J. (P-9)

KING, N. S. P. (P-15)

DONNERT, H. J. (MP-DO)

$31-8$

DEVELOPMENT OF PHOTOCONDUCTIVE POWER

SWITCH TECHNOLOGY - JULY 1983

MARCH 1984.

LOS ALAMOS,

1984. 19P. (LA-10165-PR)

NUNNALLY, WILLAM C. (E-11)

31-10

ENERGY RESPONSE AND DOSE - RATE

CALIBRATION OF A GEIGER-MUELLER

GAMMA - RAY DETECTOR.

TIC.

1984. 8P. MN (LA-UR-83-3398)

PLASSMANN, EUGENE A. (Q-2)

PEDERSON, RAYMOND A. (Q-2)

MOSS, CALVINE. (Q-2)

31-11

FABRY - PEROT INTERFEROMETRY USING AN

IMAGE - INTENSIFIED ROTATING - MIRROR

STREAK CAMERA.

HIGH SPEED PHOTOGRAPHY, VIDEOGRAPHY, AND PHOTONICS, 1983, SAN DIEGO.

PROC., P.186-91. SPIE, 1983.

(78.37/H638SP/1983)

SEITZ, WENDELL LEE (M-1)

STACY, HOWARD L. (M-1)

31-12

FAST PARALLEL ENCODING SCHEME FOR THE ANGER CAMERA.

POSITION-SENSITIVE DETECTION OF

THERMAL NEUTRONS, P. CONVERT \& J.B

FORSYTH, ED., P.188-91. ACADEMIC

PRESS, 1983. (539.77/P855SE/1982)

SEEGER, PHILIP A. (P-B)
31-13

FAST PARALEL ENCODING SCHEME FOR THE ANGER CAMERA.

IEEE TRANS. NUCL. SCI.,

V.NS-31, P.274-80. 1984

SEEGER, PHILIP A. (P-8)

31-14

FIBER OPTIC SENSOR APPLICATIONS IN

FIELDTESTING.

TIC.

1984. 23P. MN (LA-UR-64-1395)

PEREA, JACOB A. (WX-10)

31-15

GAMMA-FAY TO CERENKOV-UGHT CONVERSION EFFICIENCY FOR PURE-SILICA-CORE

OPTICAL FIBERS.

FIBER OPTICS IN ADVERSE ENVIRONMENTS

II, 1984, SAN DIEGO. PROC., P.10-7.

SPIE, 1984. (621.369/F443OP/1984)

PRUETT, B. L.

PETERSON, R.T.

SMTTH, D.E.

LOONEY, LARRY D. (P-14)

SHELTON, R. N. (AP-4)

31-16

GAS CERENKOV DETECTOR FOR MEASUAING

16.7 MEV GAMMA RAYS FROM THE

D(TRITON, GAMMA) FIVE HELIUM REACTION.

LOS ALAMOS CONFERENCE ON OPTICS '83,

PROC., P. 256-60. SPIE, 1983.

(535/L879OP/1983)

ALSO PUBUSHED IN:

TIC,

1983. 6P. MN (LA-UR-63-1864)

BROLLE, JOHN E., JR. (P-14)

LADISH, JOSEPH S. (P-14)

LYONS, PETER B. (P-14)

MACK, JOSEPH M., JP. (X-5)

TOEVS, J. W. (P-14)

YOUNG, CARLTON S. (P-14)

NERSON, STEVEN G.

NASH, P.E.

ZAGARINO, P.A.

JENNINGS, LEROY

SENO, R.D.

31-17

HIGH - SPEED VDEOGRAPHY COMEINED WITH ANX-RAY IMAGE INTENSIFIER FOR DYNAMIC

RADIOGRAPHY.

HIGH SPEED PHOTOGRAPHY, VIDEOGRAPHY, AND PHOTONICS, 1983, SAN DIEGO.

PROC., P.110-4. SPIE, 1983.

(778.37/H638SP/1983)

ALSO PUBLISHED IN:

तic.

1983. 5P. MN (LA-UR-63-2399)

BRYANT, LAWRENCE E., JR. (WX-3) 
31-18

HIGH - SPEED VIDEOGRAPHY FOR OPTICAL

AND $X$ - RAY IMAGING.

TIC,

1983. 5P. MN (LALP-83-044)

BRYANT, LAWRENCE E., JP. (WX-3)

31-19

HIGH SPEED ELECTRONIC IMAGING

APPLICATION IN AEROBALLISTIC RESEARCH HIGH SPEED PHOTOGRAPHY, VIDEOGRAPHY, AND PHOTONICS, 2ND, 1984, SAN DIEGO.

PROC., P.95-108. SPIE, 1984.

(778.37/H638SP/1984)

BROWN, R. R. (E-8)

PARKER, JOSEPH R. (P-5)

31-20

HIGH-AESOLUTION SIT TV TUBE FOR

SUBNANOSECOND IMAGE SHUTTERING.

LOS ALAMOS,

1984. 75P. (LA-09771-MS)

YATES, GEORGE J. (P-15)

VINE, BENJAMIN $H$.

AEBY, IAN

DUNBAR, DOUGLAS L. (P-14)

KING, N.S. P. (P-15)

JARAMILLO, STEVEN A. (P-15)

THAYER, NINA N.

NOEL, BRUCE W. $(W X-10)$

31-21

INTEGRATED PICOSECOND PHOTOCONDUCTORS

PRODUCED ON BULK SILICON SUBSTRATES.

BULK SILICON SUBSTRATES.

APPL. PHYS. LETT.

V.45, P.404-5. 1984.

HAMMOND, ROBERT B. (E-11)

PAULTER, NICHOLAS G. (E-11)

WAGNER, RONALD S. (E-11)

EISENSTADT, $\mathbf{W} . \mathbf{R}$.

31-22

LECTURE 1 - INTRODUCTION TO POWER

CONDITIONING SYSTEMS.

TIC,

1980. 59P. MN (LA-UR-80-0515)

SARJEANT, WALTER J. (E-4)

31-23

LECTURE 2 - DC POWER SUPPLES AND

HARD-TUBE POWER CONDITIONING SYSTEMS.

TIC,

1984. 98P. MN (LA-UR-80-0516)

SARJEANT, WALTER J. (E-4)

31-24

LOW - NOISE AMPLIFIER FOR RESOLVING THE RIGHT-LEFT AMBIGUITY IN DRIFT

CHAMBERS.

NUCL. INSTRUM. METHODS PHYS. RES.,

V.226, P.405-10. 1984.

BROWN, DAVID (E-5)

31-25

MULTIPLE - INDEPENDENT DETECTOR

SYSTEM.

IEEE TRANS. NUCL. SCI.,

V.NS-31, P.160-1. 1984

SEEGER, PHILIP A. (P-8)
31-26

NANOSECOND IMAGE SHUTTERING STUDIES AT LOS ALAMOS NATIONAL LABORATORY.

TIC,

1983. 7P. MN (LA-UR-63-3042)

YATES, GEORGE J. (P-15)

KING, N.S.P. (P-15)

JARAMILLO, STEVEN A. (P-15)

NOEL, BRUCE W. (WX-10)

GOBBY, PETER L. (MST-6)

AEBY, IAN

DETCH, J. LEWIS, JR.

31-27

NEW GENERATION LOW POWER RADIATION

SURVEY INSTRUMENTS.

IEEE TRANS. NUCL. SCI.

V.NS-31, P.655-8. 1984

WAECHTER, DAVID A. (E-7)

BJARKE, GEORGE $\mathrm{O}$. (H-1)

WOLF, MICHAEL ALLEN (H-4)

TRUJILLO, FAUSTIN (H-4)

UMBARGER, C. JOHN (H-4)

31-28

NEW GENERATION OF RADIACS - SMALL COMPUTERIZED MULTIPURPOSE RADIATION MONITORS.

TIC,

1982. 8P. MN (LA-UR-82-2980)

UMBARGER, C. JOHN (H-4)

BJARKE, GEORGE $0 .(H-1)$

ERKKILA, BRUCE H. $(\mathrm{H}-4)$

TRUJILLO, FAUSTIN (H-4)

WAECHTER, DAVID A. (E-7)

WOLF, MICHAEL ALLEN (H-4)

31-29

OBSERVED CIRCUIT LIMITS TO TIME

RESOLUTION IN CORRELATION MEASUREMENTS

WITH SILICON-ON-SAPPHIRE, GALLIUM

ARSENIC, AND INDIUM PHOSPHORUS

PICOSECOND PHOTOCONDUCTORS.

APPL. PHYS. LETT.,

V.45, P.289-91. 1984

HAMMOND, ROBERT B. (E-11)

PAULTER, NICHOLAS G. (E-11)

WAGNER, RONALD S. (E-11)

31-30

OPTICAL CHARACTERIZATION OF

RADIATION - RESISTANT FIBERS.

FIBER OPTICS IN ADVERSE ENVIRONMENTS,

2ND, 1984, SAN DIEGO. PROC.

P.22-8. SPIE, 1984

(621.369/F443OP/1984)

SMILEY, $V$.

WHITCOMB, BRUCE $M$.

PERRASSINI, $M$

WHITAKER, D.

FLURER, RANDY L

COLBURN, CHRISTOPHER W.

LYONS, PETER B. (P-DO)

OGLE, J. W. (P-14)

LOONEY, LARRY D. (P-14)
31-31

OPTIMAL OBJECT AND EDGE LOCALIZATION

IN THE PRESENCE OF CORRELATED NOISE.

TIC,

1984. 10P. MN (LA-UR-84-0984)

HANSON, KENNETH M. (M-4)

31-32

PORTABLE BETA SPECTROMETER - DOSIMETER.

IEEE TRANS. NUCL. SCI.

V.NS-31, P.661-3. 1984.

ERKKILA, BRUCE H. (H-4)

WAECHTER, DAVID A. (E-1)

BRAKE, RICHARD JOSEPH (HSE-1)

31-33

PORTABLE ELECTROCHEMICAL CELI

INTERROGATOR.

LOS ALAMOS,

1984. 7P. (LA-10274-MS)

DOSS, JAMES D. (MP-3)

MC CABE, CHARLES W. (MP-3)

31-34

PULSED FLUORESCENT X - RAY SYSTEM WITH

ON - LINE DIGITIZER AND DATA PROCESSOR.

LOS ALAMOS

1984. 12P. (LA-10247)

BERZINS, GEORGE J. (ESS-9)

VALENCIA, JOHN E. (ESS-11)

GUTIERREZ, JOSE W. (ESS-11)

MC GIRT, A. FRANK (ESS-10)

MOORE, KURT R. (ESS-10)

31-35

PULSED SOFT X-RAY RESPONSE OF INDIUM

PHOSPHORUS-IRON PHOTOCONDUCTORS.

APPL. PHYS. LETT.,

V.44, P.1059-61. 1984

KANIA, DON R. (P-14)

BARTLETT, ROGER J. (P-14)

WAGNER, RONALD S. (E-11)

HAMMOND, ROBERT B. (E-11)

PIANETTA, $P$.

31-36

RAILGUN CONDUCTOR HEATING FROM MULTIPLE

CURRENT PULSES.

LOS ALAMOS

1984. 26P. (LA-10184-MS)

KERRISK, JERRY F. ( $(W X-4)$

31-37

SMART INSTRUMENTATION DEVELOPMENT AT

LOS ALAMOS.

TIC.

1984. 12P. MN (LA-UR-83-3409)

ERKKILA, BRUCE H. (IT-1) 
$31-38$

SPECTROMETER FOR INELASTIC SCATTERING

USING NEUTRONS FROM 1 EV TO $186 \mathrm{EV}$.

NUCL. INSTRUM. METHODS,

V.221, P.393-407. 1984.

BRUGGER, ROBERT M. (P-8)

TAYLOR, ANDREW D. (P-8)

OLSEN, CLAYTON E. (MST-13)

GOLDSTONE, JOYCE A. (P-8)

SOPER, ALAN K. (P-8)

31-39

SURVEY OF FLASHX - RAY TECHNIOUES AND APPLICATIONS.

HIGH SPEED PHOTOGRAPHY, VIDEOGRAPHY, AND PHOTONICS, 1983, SAN DIEGO.

PROC. P.16-21. SPIE, 1983.

(778.37/H638SP/1983)

ALSO PUBLISHED IN:

TIC.

1983. 7P. MN (LA-UR-83-2400)

BRYANT, LAWRENCE E., JR. (WX-3)

LUCERO, J. P. (M-5)

$31-40$

TRANSIENT ATTENUATION IN OPTICAL

FIBERS.

FIBER OPTICS IN ADVERSE ENVIRONMENTS, 2ND, 1984, SAN DIEGO. PROC.

P.209-16. SPIE, 1984.

(621.369/F443OP/1984)

HOPKINS, ANNA (P-14)

KELLY, R.E.

LOONEY, LARRY D. (P-14)

LYONS, PETER B. (P-14)
$31-41$

UNUSUAL CHEMICAL COMPOSITION FLUORESCERS VIOUNIST - SOPHISTICATED PLUTONIUM

AND FILTERS FOR LOW-ENERGY X-RAY

DIAGNOSTICS - PART I. OXYGEN

COMPOSITIONS.

REV. SCI. INSTRUM.,

V.55, P.35-8. 1984.

JORGENSEN, BETTY S. (MST-6)

LIEPINS, RAIMOND (MST-6)

DAY, ROBERT H. (P-14)

31-42

USING PHOTOCONDUCTIVITY TO IMPROVE IMAGE TUBE GATING SPEEDS.

TIC,

1983. 4P. MN (LA-UR-83-3060)

GOBBY, PETER L (MST-6)

YATES, GEORGE J. (P-15)

JARAMILLO, STEVEN A. (P-15)

NOEL, BRUCE $W .(W X-10)$

AEBY, IAN
31.43

FIELD MONITOR.

IEEE TRANS. NUCL. SCI.,

V.NS-31, P.659-60. 1984

UMBARGER, C. JOHN (H-4)

WOLF, MICHAEL ALLEN (H-4)

$31-44$

100 MHZ FIBER OPTIC SINGLE TRANSIENT

GAMMA RAY DETECTION SYSTEM.

LONG-HAUL MEASUREMENTS AND

APPLICATIONS, 2ND, 1984, SAN DIEGO.

SPIE, 1984. (621.369/F443OPT/1984)

OGLE, J. W. (P-14)

SMITH, RUSS C. (Q-7)

WARD, $M$.

RAMSEY, $R$.

HOLLABAUGH, J. S.
TRUJILLO, FAUSTIN (H-4)

FIBER OPTICS - SHORT-HAUL AND 


\section{ISOTOPE SEPARATION}

32-1

ABSORPTION SPECTRUM OF PLUTONIUM

HEXAFLUORIDE IN THE $3000-9000$

A SPECTRAL REGION.

SPECTROCHIM. ACTA,

V.4OA, P.587-9. 1984.

WALTERS, RAYMONDT.

BRIESMEISTER, RICHARD A. (AP-4)

32-2

DAMAGE THRESHOLDS TO METAL MIRRORS BY

SHORT - PULSE CARBON DIOXIDE LASER

RADIATION.

LASER INDUCED DAMAGE IN OPTICAL

MATERIALS, 13TH, 1981, BOULDER.

PROC., P.229-38. NBS, 1983.

(620.112/S9890M/13TH/1981)

FIGUEIRA, JOSEPH F. (AP-5)

THOMAS, SCOTT J. (AP-5)

HARRISON, RONALD F. (AP-5)
$32-3$

METHOD OF SEPARATIN BORON ISOTOPES. PATENTED

MAY 8, 1984, FILED 1981.

(U.S. PATENT-4,447,303)

JENSEN, REED J. (P-DO)

THORNE, JAMES $M$.

CLUFF, CORAN L.

HAYES, JOHN K. (ADWP-2)
$32-4$

MULTIPLE - SHOT ULTRAVIOLET LASER DAMAGE RESISTANCE OF NONQUARTERWAVE REFLECTOR DESIGNS FOR 248 NM.

LASER INDUCED DAMAGE IN OPTICAL

MATERIALS, 13TH, 1981, BOULDER.

PROC., P.363-371. NBS. 1983.

(620.112/59890M/13TH/1981)

ALSO PUBLISHED IN:

TIC.

1982. 20P. MN (LA-UR-82-1774)

NEWNAM, BRIANE. (AP-2)

FOLTYN, STEPHEN R. (AP-2)

JOLIN, L J. (AP-2) 


\section{LIGHT WATER REACTOR TECHNOLOGY}

33-1

ANALYSIS OF UNMITIGATED BORON DILUTION EVENTS IN PRESSURIZED WATER REACTORS DURING SHUTDOWN.

TRANS. AM. NUCL. SOC.,

V.46, P.254-6. 1984. NASSERSHARIF, BAHRAM (Q-7)

WING, J. E. (J-15)

33-2

ASSESSMENT OF THE UTILTYY OF FEEDAND - BLEED OPERATING MAPS.

TRANS. AM. NUCL. SOC.,

V.47, P.263-6. 1984.

BOYACK, B. E. (Q-7)

33-3

FAST NUMERICAL METHODS IN 3 - D.

TIC.

1984. 11P. MN (LA-UR-84-1721)

WOODRUFF, SUSAN B. (Q-8)

$33-4$

HMS - A MODEL FOR HYOROGEN MIGRATION

STUDIES IN LWR CONTAINMENTS.

INTL CONF. ON THE IMPACT OF HYDROGEN ON WATER REACTOR SAFETY, 2ND, 1982,

ALBUQUERQUE. PROC., P.489-508. (NUREG/CP-D038)

TRAVS, JOHN R. (T-3)

33-5

HYOROGEN DIFFUSION FLAMES BURNING IN A

MARK-III CONTAINMENT DESIGN.

TIC.

1984. 33P. MN (LA-UR-84-1319)

TRAVIS, JOHN R. (T-3)

33-6

HYDROGEN MIXING STUDY (HMS) IN LWR

TYPE CONTAINMENTS

TIC,

1983.21P. MN (LA-UR-83-3451)

TRAVIS, JOHN R. (T-3)

33-7

HYDROGEN TRANSPORT, MIXING, AND

COMBUSTION STUDIES.

TIC,

1984. 16P. MN (LA-UR-84-1649)

TRAVIS, JOHN R. (T-3)

33-8

INTERFACING SYSTEMS LOSS - OF - COOLANT ACCIDENT IN OCONEE UNIT I PRESSURIZED

WATER REACTOR.

TRANS. AM. NUCL. SOC.

V.47, P.258-60. 1984.

NASSERSHARIF, BAHRAM (Q-7)
33-9

LOFW - INITIATED ATWS ANALYSIS FOR

OCONEE - 1 USING TRAC - PF1/MOD1.

TRANS. AM. NUCL. SOC.,

Y.47, P.260-1. 1984.

HENNINGER, RUDOLPH J. (Q-7)

JENKS, RICHARD P. (Q-7)

33-10

NEUTRON MEASUREMENTS IN BOORATED WATER

FOR PWR FUEL INSPECTIONS.

LOS ALAMOS,

1984. 68P. (LA-10068-MS)

RINARD, PHILLIP M. (Q-1)

33-11

NUCLEAR PLANT ANALYZER DEVELOPMENT AT

LOS ALAMOS.

INTL. NUCLEAR POWER PLANT THERMAL

HYDRAULCS AND OPERATIONS TOPICAL

MEETING, 1984, TAIPEI. PROC.,

P.H7-1-8. AMER. NUCLEAR SOCIETY,

1984. (621.483/161NU/1884)

LILES, DENNIS R. (Q-9)

GIGUERE, PAUL T. (Q-9)

33-12

PWR SHUTDOWN DECAY HEAT REMOVAL

ANALYSES IN SUPPORT OF TAPA - 45.

TIC.

1983. 13P. MN (LA-UR-83-3116).

BOYACK, B. E. (Q-7)

HENNINGER, RUDOLPH J. (Q-7)

SMITH, RUSS C. (Q-7)

33-13

REACTOR VITAL EQUIPMENT DETERMINATION TECHNIQUES.

TIC,

1983. 9P. MN (LA-UR-83-3026)

BOT, T.F. (Q-7)

THOMAS, W. 5.

33-14

SMALL - BREAK LOCA RECOVERY IN B\&W

PLANTS.

TIC.

1983. 13P. MN (LA-UR-83-2786)

HENNINGER, RUDOLPH J. (Q-7)

IRELAND, JOHN R. (Q-7)

DEMUTH, NELSON S., JR. (Q-7)

33-15

THREE-DIMENSIONAL THERMALHYDRAULIC CALCULATIONS USING SOLA-PTS.

TIC,

1983. 2BP. MN (LA-UR-83-3062)

DALY, BART J. (T-3)

TORREY, MARTIN D. (T-3)
33-16

TRAC - PF1 - PRESSURIZED THERMAL SHOCK CALCULATIONS FOR SEVERAL SMALL - BREAK LOSS - OF - COOLANT ACCIDENT TRANSIENTS IN THE OCONEE - 1 NUCLEAR POWER PLANT.

TIC,

1983. 17P. MN (LA-UR-83-2816)

BURKETT, MICHAEL W. (Q-6)

BASSETT, BRITT (O-T)

33-17

TAAC - PFI ANALYSIS OF LOFT STEAM-

GENERATOR FEEDWATER TRANSIENT TEST Le-1.

Tic,

1983. 10P. MN (LA-UR-83-2780)

MEIER, JOHN K. (Q-9)

33-18

TRAC - PF1 STUDY OF LOSS OF PUMP SEALS

IN A WESTINGHOUSE PWR.

TRANS. AM. NUCL. SOC.,

V.46, P.472-3. 1984.

BARTS, EDWARD W. (Q-6)

BOTT, T. F. (Q-7)

33-19

TRAC-PF1/MOD1 COMPUTER CODE.

TIC.

1983. 7P. MN (LA-UR-83-2396)

LILES, DENNIS R. (Q-9)

MAHAFFY, JOHN H. (Q-9)

33-20

TRAC ANALYSES FOR CCTF AND SCTF TESTS AND UPTF DESIGN/OPERATION.

TIC,

1983. 27P. MN (LA-UR-83-2949)

WILLIAMS, KEN A. (Q-8)

33-21

TRAC ANALYSIS AND SUPPORT OF OCONEE - 1

PTS STUDIES.

TIC,

1983. 15P. MN (LA-UR-83-3011)

IRELAND, JOHNR. (Q-7)

33-22

TRAC ANALYSIS OF THE CRYSTAL RIVER

UNIT - 3 PLANT TRANSIENT OF FEBRUARY 26, 1980.

nc.

1983. 12P. MN (LA-UR-83-2719)

CODDINGTON, PAUL

WILLCUTT, GORDON J., JA. (Q-7) 
33-23

TRAC CALCULATIONS OF OVERCOOLING

TRANSIENTS IN PWRS FOR PRESSURIZED

THERMAL SHOCK ANALYSIS.

AMERICAN NUCLEAR SOCIETY TOPICAL

MEETING ON ANTICIPATED AND ABNORMAL

PLANT TRANSIENTS IN LIGHT WATER

REACTORS, 1983, JACKSON.

PROC., P.587-601. PLENUM, 1984.

ALSO PUBLISHED IN:

TIC,

1983. 16P. MN (LA-UR-83-2753)

IRELAND, JOHN R. (Q-7)

BOYACK, B. E. (Q-7)
$33-24$

TRAC INDEPENDENT ASSESSMENT FOR PWR ANALYSIS.

TIC,

1983. 14P. MN (LA-UR-63-3127) KNIGHT, THAD D. (Q-9)
33-25

TRAC-PF1 POST-TEST PREDICTIONS FOR THE

SEMISCALE NATURAL - CIRCULATION TESTS

S - NC -2 AND S - NC - 6 .

TIC.

1983. 13P. MN (LA-UR-83-2806) BOOKER, C. P. (Q-9) 
35. LMFBR-FUELS AND MATERIALS ENGINEERING AND DEVELOPMENT: BASE TECHNOLOGY

35-1

EXAMINATION OF FAST REACTOR FUELS AND FBR ANALYTICAL QUALTTY ASSURANCE STANDARDS AND METHODS, OCTOBER 1. DECEMBER 31, 1983

LOS ALAMOS,

1984. 4P. (LA-10036-PR)

WOOD, WARREN T. (MST-14)
35-2

EXAMINATION OF FAST REACTOR FUELS AND

FBR ANALYTICAL QUALTTY - ASSURANCE

STANDARDS AND METHODS, JANUARY 1-

MARCH 31, 1984.

LOS ALAMOS,

1984. 4P. (LA-10120-PR)

DOWLER, KEITHE. (MST-14)
35-3

SLIDELINE VERIFICATION FOR MULTILAYER PRESSURE VESSEL AND PIPING ANALYSIS INCLUDING TANGENTIAL MOTION.

TIC,

1984. 26P. MN (LA-UR-63-3514)

VAN GULICK, LEONARD A. (WX-11) 
36. LMFBR-SAFETY TECHNOLOGY

36-1

INTERSUBASSEMBLY FUEL PENETRATION INTO A FAILED PRIMARY CONTROL SUBASSEMBLY IN AN LMFBR.

LOS ALAMOS,

1984. 27P. (LA-09952-MS)

DE VAULT, GUILLAUME P. (Q-7)

$36-2$

RADIAL POWER SHIFTS IN HETEROGENEOUS FAST REACTOR CALCULATIONS.

TRANS. AM. NUCL. SOC.

V.46, P.502-3. 1984.

ASPREY, MARGARET $W .(\alpha-7)$ 
37-1

ADVANCEO NUCLEAR DATA FOR RADIATION

DAMAGE CALCULATIONS.

TIC.

1983. 7P. MN (LA-UR-83-2628)

MAC FARLANE, ROBERT E. (T-2)

FOSTER, D. GRAHAM, JR. (T-2)

37-2

CERAMICS FOR FUSION DEVICES.

J. MATER. ENERGY SYST.

V.6, P.100-5. 1984

ALSO PUBLISHED IN:

TiC,

1984. OP. MN (LA-UR-84-1354)

CLINARD, FRANK W., JR. (MST-5)

$37-3$

DESCRIPTION AND PERFORMANCE OF URANIUM

BEDS USED TO PUMP TRITIUM-DEUTERIUM AT.

THE TRITIUM SYSTEMS TEST ASSEMBLY.

ASSEMBLY.

J. VAC. SCI. TECHNOL.

V.2A, P.722-3. 1984.

ALSO PUBLISHED IN:

ThC.

1983. 5P. MN (LA-UR-83-2694)

WALTHERS, CHARLES R. (MST-3)

$37-4$

DESIGN AND OPERATION OF THE $30 \mathrm{MJ}$

SUPERCONDUCTING MAGNETIC STORAGE SYSTEM

ON THE BONNEVILLE POWER ADMINISTRATION

Bus.

TIC.

1983. 11P. MN (LA-UR-63-2594)

ALSO PUBLISHED IN:

ADV. CRYOG. ENG.,

V.29.P.123-32. 1984.

SCHERMER, ROBERT I. (CTR-9)

- BARRON, MICHAEL H. (CTR-9)

BOENIG, HEINRICH J. (CTR-8)

BROWN, R. R. (E-5)

CRISCUOLO, ALPHONSE L. (E-8)

CUMMING, COUN J. (E-8)

DEAN, JOHNW. (CTR-9)

HASSENZAHL, WILLIAM V. (CTR-9)

ROGERS, JOHN D., JR. (CTR-9)

SEAMONS, MAX J. (E-B)

TURNER, ROBERT D. (CTR-9)

WHITFILL, JAMES A. (E-5)

CHASTAIN, R. E.

COLEMAN, J. G

HANER, J. M.

HAUER, J.F.

MILLER, B.L.

SCHAAD, P.A.

ALCORN, J.S.

HOFFMAN, E. H.

PURCELL, JOHN R.

\section{MAGNETIC FUSION ENERGY}

37-5

MATERIALS NEEDS FOR COMPACT FUSION

REACTORS.

TIC,

1983. 15P. MN (LA-UR-83-2570)

KRAKOWSKI, ROBERT A. (CTR-12)

$37-6$

METHOD AND APPARATUS FOR FAST LASER

PULSE DETECTION USING GASEOUS PLASMAS.

PATENTED

MAY 8, 1984, FILED 1981.

(U.S.PATENT-4,447,151)

MC LELLAN, EDWARD J. (AP-5)

WEBB, JOHN A. (L-9)

37-7

PLANT AND OPERATIONAL FEATURES OF THE

BPA $30 \mathrm{MJ}$ SUPERCONDUCTING MAGNETIC

ENERGY STORAGE SYSTEM.

TIC.

1984. 7P. MN (LA-UR-64-0052)

ROGERS, JOHN D., JR. (CTR-9)

HAUER, J.F.

$37-6$

RADIATION - INDUCED RF LOSS MEASUREMENTS AND THERMAL STRESS CALCULATIONS FOR CERAMIC WINDOWS.

TIC,

1983. 7P. MN (LA-UR-83-2783)

FOWLER, J. D. (MST-5)

37-9

SOME CONSIDERATIONS ON SILICONE OIL IN HIGH CURRENT AND ENERGY DISCONNECTING MECHANISMS

LOS ALAMOS,

1984. 9P. (LA-10035-MS)

VOGEL, HERBERT F. (CTR-4)

37-10

STRUCTURAL PERFORMANCE OF CERAMICS IN A HIGH - FLUENCE FUSION ENVIRONMENT.

J. NUCL MATER.

V.122-3, P.1386-92. 1984.

ALSO PUBLISHED IN

TIC.

1983. 8P. MN (LA-UR-83-2742)

CLINARD, FRANK W., JR. (MST-5)

HURLEY, GEORGE F. (MST-5)

HOBBS, LNN W. (MST-5)

ROHR, DANAL L (MST-5)

YOUNGMAN, ROBERT A. (MST-5)
37-11

STUDY OF DEFECTS PRODUCED IN TUNGSTEN BY

800 - MEV PROTONS USING FIELD ION

MICROSCOPY.

TIC.

1983. 7P. MN (LA-UR-83-2572)

FARNUM, DAVID J. (MP-13)

SOMMER, WALTER F., JR. (MP-13)

INAL, OSMAN T.

37-12

SUPERCONDUCTING MAGNETIC ENERGY STORAGE (SMES) PROGRAM, JANUARY 1 - DECEMBER 31, 1983.

LOS ALAMOS,

1984. 24P. (LA-10050-PR)

ROGERS, JOHN D., JR. (CTR-9)

37-13

TESTS OF THE $30 \mathrm{MJ}$ SUPERCONDUCTING

MAGNETIC ENERGY STORAGE UNIT.

TIC,

1983. 7P. MN (LA-UR-83-2516)

BOENIG, HEINRICH J. (CTR-9)

DEAN, JOHN W. (CTR-9)

ROGERS, JOHN D., JR. (CTR-9)

SCHERMER, ROBERT I. (CTR-9)

HAUER, J.F.

37-14

30 MJ SMES SYSTEM OPERATING EXPERIENCE, NOVEMBER 1982 - JUNE 1983.

DOE PHYSICAL AND CHEMICAL ENERGY

STORAGE ANNUAL CONTRACTORS' REVIEW

MEETING, 1983, ARLINGTON. PROC.,

P.189-94. DOE, 1983. (CONF-830974)

ALSO PUBLISHED IN:

THC,

1883. 7P. MN (LA-UR-83-2395)

SCHERMER, ROBERT I. (CTR-9)

37-15

$30 \mathrm{MJ}$ SUPERCONDUCTING MAGNETIC ENERGY STORAGE STABIUZING SYSTEM - AN

OVERVIEW.

DOE PHYSICAL AND CHEMICAL ENERGY

STORAGE ANNUAL CONTRACTORS' REVIEW

MEETING, 1983, ARLINGTON. PROC.,

P.184-88. DOE, 1983. (CONF-630974)

ALSO PUBLSSHED IN:

TIC,

1983. 6P. MN (LA-UR-83-2343)

ROGERS, JOHN D., JR. (CTR-9)

BOENIG, HEINRICH J. (CTR-9)

DEAN, JOHN W. (CTR-9)

SCHERMER, ROBERT I. (CTR-9)

ANNESTRAND, S. A

HAUER, J.F.

MILLER, B.L 


\section{MATERIALS}

38-1

AUGER AND DEPTH PROFILE ANALYSIS OF SYNTHETIC CRYSTALS FOR DISPERSION OF THE SOFT $X$ - RAYS.

APPL. SURF. SCI.,

V.18, P.165-81. 1984.

ALSO PUBLISHED IN:

TIC,

1983. 25P. MN (LA-UR-83-1965)

RACHOCKI, KENNETH D. (MST-5)

BROWN, DAVID R. (MST-6)

SPRINGER, ROBERT W. (MST-6)

ARENDT, PAUL N. (MST-6)

38-2

BIAXIAL DEFORMATION OF 70 - 30 BRASS -

FLOW BEHAVIORS, TEXTURE,

MICROSTRUCTURES.

METALL. TRANS.,

V.15A, P.1607-12. 1984.

STOUT, MICHAEL G. (MST-5)

STAUDHAMMER, KARL P. (MST-5)

38-3

CALCULATION OF DISPLACEMENT AND HELIUM PRODUCTIONAT THE LAMPF IRRADIATION FACILITY.

TIC,

1984. 18P. MN (LA-UR-84-1124)

DAVIDSON, D. R. (MP-13)

GREENWOOD, L. R.

SOMMER, WALTER F., JR. (MP-13)

WECHSLER, M. S. (MP-3)

38-4

CERAMIC - CERAMIC SEALS BY MICROWAVE HEATING.

TIC,

1984. 15P. MN (LA-UR-84-0541)

MEEK, THOMAS T. (MST-6)

BLAKE, RODGER D. (MST-6)

38-5

CHARACTERIZATION OF A SAMPLE OF VERY

PURE HAFNIA.

LOS ALAMOS. "

1984. 4P. (LA-09930)

SKAGGS, SAMUEL R. (CMB-3)

GAC. FRANK D. (MST-6)

MAGNUSON, JOHNE. (MST-6)
38-6

CLOSE LOOKAT LOW - TEMPERATURE

MAGNETIZATION DATA FOR

TITANIUM BERYLLIUM 2.

J. MAGN. MAGN. MATER.,

V.46, P.11-8. 1984.

ACKER, FLORENTIN

HUGUENIN, $R$.

PELIZZONE, $M$

SMITH, JAMES L. (MST-5)

38-7

CREEP AND THERMAL EFFECTS IN AGING

SOLIDS.

TIC,

1983. 38P. MN (LA-UR-83-1379)

ANDERSON, CHARLES A. (Q-13)

38-8

DEVELOPMENT OF PLUTONIUM TRANSMISSION

ELECTRON MICROSCOPY.

LOS ALAMOS,

1984. 11P. (LA-09965-MS)

ROHR, DANA L. (MST-5)

STAUDHAMMER, KARL P. (MST-5)

JOHNSON, KAYE ALLAN (MST-13)

38-9

DEVELOPMENT OF THE VLS PROCESS FOR

SIC WHISKER GROWTH.

TIC,

1984. 20P. MN (LA-UR-83-3717)

HURLEY, GEORGE F. (MST-5)

38-10

EFFECTS OF NEUTRON IRRADIATION ON

MAGNETIC PERMEABILTY OF AMORPHOUS AND

CRYSTALLINE MAGNETIC ALLOYS.

J. APPL. PHYS.,

V.55, P.1754-6. 1984

BROWN, ROBERT D. (MP-7)

COST, JAMES RICHARD (MST-5)

STANLEY, JAMES T. (MST-5)

38-11

EFFECTS OF SUPERCONDUCTIVITY ON RARE-

EARTH DYNAMICS IN (HOLIUM LUTETIUM)

RHODIUM BORIDES.

J. APPL. PHYS

V.55, P.2007-9. 1984.

ALSO PUBLISHED IN:

TIC,

1983. 15P. MN (LA-UR-83-2775)

HEFFNER, ROBERT H. (MP-3)

COOKE, D.W.

HUTSON, RICHARD L (MP-3)

LEON, MELVIN (MP-3)

SCHILLACI, MARIO E (MP-3)

SMITH, JAMES L. (MST-5)

YAOUANC, ALAIN (MP-3)

DODDS, STANLEY A.

GUPTA, LAXMI C.

MACLAUGHLN, DOUGLASE

BOEKEMA, CAROLUS (MP-3)
38-12

ELECTRON BEAM DIAGNOSTIC FOR THE LOS ALAMOS FREE - ELECTRON LASER OSCILLATOR EXPERIMENT.

LINEAR ACCELERATOR CONFERENCE,

1984, SEEHEIM. PROC., P.436-8.

1984. (GSI-84-11)

ALSO PUBLISHED IN:

TIC,

1984. 4P. MN (LA-UR-84-1191)

SHEFFIELD, RICHARD L. (AT-7)

38-13

EXCITATION AND IRON CONCENTRATION

DEPENDENCES IN THE IMPULSE

PHOTOCONDUCTANCE OF INDIUM PHOSPHROUS IRON.

APPL. PHYS. LETT.,

V.44,P.620-2. 1984.

HAMMOND, ROBERT B. (E-11)

PAULTER, NICHOLAS G. (E-11)

WAGNER, RONALD S. (E-11)

SPRINGER, THOMAS E. (E-11)

38-14

FRACTURE TOUGHNESS OF A BETA -

SILICON CARBIDE WHISKER.

J. AM. CERAM. SOC.,

V.67, P.C219-20. 1984.

PETROVIC, JOHN J. (MST-5)

ROOF, RAYMOND BRADLEY, JR. (MST-5)

38-15

HEAYY-FERMION SYSTEMS

REV. MOD. PHYS.

V.56, P.755-84. 1984.

STEWART, GREGORY R. (MST-5)

38-16

HENRY'S LAW VAPORIZATION STUDIES ANO

THERMODYNAMICS OF EINSTEINIUM - 253

METAL DISSOLVED IN YTTERBIUM.

J. CHEM. PHYS.

V.81, P.473-7. 1984.

KLEINSCHMIDT, PHILLIP D. (MST-5)

WARD, JOHN W. (MST-13)

MATLACK, GEORGE M. (CHM-1)

HAIRE, RICHARD G.

38-17

HIGH-FIELD SPECIFIC HEATS OF A15

VANADIUM SILICON AND NIOBIUM TIN

PHYS. REV.,

V.29B, P.3908-12. 1984

STEWART, GREGORY R. (MST-5)

BRANDT, BRUCE L 
$38-18$

IMPÚRITIES IN THE HEAVY-FERMION

SUPERCONDUCTOR URANIUM BERYLIUM 13.

TIC,

1983. 20P. MN (LA-UR-83-3064)

ALSO PUBLISHED IN:

J. APPL. PHYS.

V.55, P.1996-9. 1984.

SMITH, JAMES L. (MST-5)

FISK, ZACHARY (MST-5)

WILLIS, JEFFREY O. (MST-5)

BATLOGG, B.

OTT, HANS R.

38-19

INFLUENCE OF SPIN FLUCTUATIONS ON THE ELECTRICAL RESISTANCE OF URANIUM ALUMINUM 2 AND URANIUM PLATINUM 3 AT HIGH PRESSURES AND LOW TEMPERATURES.

PHYS. REV.,

V.30B, P.5591-6. 1984

WIRE, MIKE S. (MST-5)

THOMPSON, JOE D. (P-10)

FISK, ZACHARY (MST-5)

38-20

ISOTHERMAL CALORIMETAIC INVESTIGATION OF A REVERSIBLE REACTION IN RAPIDLY SOLIDIFIED IRON 40 NICKEL 40 BORON 20

TIC,

1983. 6P. MN (LA-UR-84-0136)

COST, JAMES RICHARD (MST-5)

ELLIOTT, REED O. (CMB-5)

STANLEY, JAMES T. (MST-5)

38-21

KINETICS OF STRUCTURAL RELAXATION IN

METALLIC GLASSES.

TIC,

1983. 7P. MN (LA-UR-83-2254)

ALSO PUBLISHED IN:

J. NON-CRYST. SOLIDS

V.61-62, P.799-804, 1984

COST, JAMES RICHARD (MST-5)

STANLEY, JAMES T. (MST -5)

38-22

LOW DENSITY, MICROCELLULAR FOAMS,

PREPARATION, AND ARTICLES.

PATENTED

FEB. 7, 1984, FILED 1982.

(U.S. PATENT-4,430,451)

YOUNG, AINSLIET., JR. (MST-6)

MARSTERS, ROBERT G. (MST-6)

MORENO, DAWN K. (CMB-10)

38-23

LOW-TEMPERATURE PROPERTIES OF THE HEAWFERMION SYSTEM URANIUM CADMIUM 11

PHYS. REV.,

V.30B, P.6360-2. 1984

FISK, ZACHARY (MST-6)

STEWART, GREGORY R. (MST-5)

WILLIS, JEFFREY O. (MST -5)

OTT, HANS R.

HULLIGER, F.
38-24

MAGNETIC SUSCEPTIBILITY, ELECTRICAL RESISTIVITY, AND SPECIFIC HEAT OF SINGLE CRYSTAL LANTHANUM IRON 4 PHOSPHORUS 12

INTERNATIONAL CONFERENCE ON LOW

TEMPERATURE PHYSICS, 17TH, 1984

KARLSRUHE. PROC., P.711-2. ELSEVIER, 1984.

MEISNER, G. P. (MST-5)

STEWART, GREGORY R. (MST-5)

TORIKACHVIU, MILTON 5.

MAPLE, MERRILL. $B$.

$38-25$

MATERIALS SCIENCE AND TECHNOLOGY

DIVISION - ORGANIZATIONAL PROFILE.

LOS ALAMOS,

1984. 6P. (LALP-83-058)

NEARY, JOAN (IS-1)

38-26

METALLOGRAPHIC GRINDING EFFICIENCY.

LOS ALAMOS

1984. 8P. (LA-10215-MS)

JOHNSON, KAYE ALLAN (MST-13)

38-27

MULTIAXIAL LOADING FRACTURE OF

ALUMINUM OXYCEN TUBES - 1 -

EXPERIMENTS.

J. AM. CERAM. SOC.,

V.67, P.14-8. 1984.

STOUT, MICHAEL G. (MST-5)

PETROVIC, JOHN J. (MST-5)

38-28

MULTIAXIAL LOADING FRACTUAE OF

ALUMINUM OXYGEN TUBES - II, WEIBULLL

THEORY AND ANALYSIS.

J. AM. CERAM. SOC.

V.67, P.18-22. 1984

PETROVIC, JOHN J. (MST-6)

STOUT, MICHAEL G. (MST-5)

38-29

MUON SPIN RELAXATION IN SPIN GLASS

PALLADIUM MANGANESE.

TIC,

1983. 15P. MN (LA-UR-83-3443)

HEFFNER, ROBERT H. (MP-3)

LEON, MELVIN (MP-3)

SCHILLACI, MARIOE. (MP-3)

DODDS, STANLEY A.

GIST, GRANT A.

MACLAUGHLIN, DOUGLAS E.

MYOOSH, JOHN A.

NIEUWENHUYS, G. J.

38-30

MUON SPIN RELAXATION

PHYS. TODAY,

V.37, P.38-45. 1984.

HEFFNER, ROBERT H. (MP-3)

FLEMING, DONALD G.
$38-31$

NEW CERIUM HEAWY FERMION SYSTEM -

CERIUM COPPER 6.

PHYS. REV.,

V.30B, P.482-3. 1984.

STEWART, GREGORY R. (MST-5)

FISK, ZACHARY (MST-5)

WIRE, MIKE S. (MST-5)

$38-32$

NEW HEAVY-FERMION SYSTEM, NEPTUNIUM

BERYLLIUM 13, WITH A COMPARISON TO

URANIUM BERYLLIUM 13 AND PLUTONIUM

BERYLLIUM 13

PHYS. REV.,

V.30B,. P.1249-52. 1984.

STEWART, GREGORY R. (MST-5)

FISK, ZACHARY (MST-5)

SMITH, JAMES L. (MST-5)

WILLIS, JEFFREY O. (MST-5)

WIRE, MIKE S. (MST-5)

$38-33$

NON-LINEAR DYNAMICS, BREATHERS AND

PHOTOINDUCED ABSORPTION IN

POLYACETYLENE

SYNTH. MET.

V.9, P.223-39. 1984

BISHOP, ALAN R. (T-11)

CAMPBELL, DAVD K. (CNLS)

LOMDAHL, PETER S. (CNLS)

HOROVITZ, BARUCH

PHILLPOT, SIMON R.

$38-34$

NOVEL HARD METAL COMPOSITIONS AND

PROPERTIES.

INTERNATIONAL SCHOOL ON SINTERED

MATERIALS, 3RD, 1983, NEW DELHI.

PROC., P.377-98. ELSEVIER, 1984.

ALSO PUBLISHED IN:

TIC,

1983. 26P. MN (LA-UR-63-3045)

SHEINBERG, HASKELL (MST-6)

38-35

NOVEL RF-PLASMA SYSTEM FOR THE

SYNTHESIS OF ULTRAFINE, ULTRAPURE

SILICON CARBIDE AND SILICON NITRIDE.

MATERIALS RESEARCH SOCIETY SYMPOSIA,

1983, BOSTON. PROC., P.283-8.

ELSEVIER, 1984

ALSO PUBLISHED IN:

TIC.

1983. 19P. MN (LA-UR-83-3169)

VOGT, GERALD J. (MST-3)

HOLLABAUGH, CHARLES M. (MST-3)

HULL, DONALDE. (MST-7)

NEWKIRK, LAWRENCE R. (MST-3)

PETROVIC, JOHN J. (MST-5) 
38-36

ON THE ORIGIN OF SHEER BANDS IN

TEXTURED POLYCRYSTALS.

SCR. METALL.

V.18, P.437-42. 1984.

CANOVA, G. R. (CMS)

KOCKS, U. FRED (CMS)

STOUT, MICHAEL G. (MST-5)

38-37

PHASE DIAGRAM AND CRITICAL POINTS OF CERIUM ALLOYS.

J. APPL. PHYS.

V.55, P.1921-4. 1984.

FIS, ZACHARY (MST-5)

THOMPSON, JOE D. (P-10)

LAWRENCE, J. M.

SMITH, JAMES L. (MST-5)

BATLOGG, B.

38-38

PHASE STABILITY AND MAGNETIC PROPERTIES

OF URANTIUM ALUMINUM SUB 2 BASED

PSEUDOBINARY SPIN FLUCTUATION COMPOUNDS.

LOS ALAMOS.

1984. 88P. (LA-10199-T)

WIRE, MIKE S. (MST-5)

$38-39$

PHONON ANOMALES IN ZIRCONIUM

RUTHENIUM PHOSPHORUS?

J. LOW TEMP. PHYS.

V.55, P.11-5. 1984.

KEIBER, HUBERT

WUHL, HELMUT

MEISNER, G. P. (MST -5)

STEWART, GREGORY R. (MST-5)

$38-40$

POLYACETYLENE FIBRIL GROWTH IN SOLUBLE

ZIEGLER-NATTA CATALYSTS.

MACROMOLECULES

V.17,P.1633-4. 1984.

- ALDISSI, MAHMOUD (MST-6)

SCHUE, F.
$38-41$

POSSIBILITY OF COEXISTENCE OF BULK SUPERCONDUCTIVITY IN URANIUM PLATINUM.

FLUCTUATIONS IN UPT3.

PHYS. REV. LETT.

V.52, P.679-81. 1984

STEWART, GREGORY R. (MST-5)

FISK, ZACHARY (MST-5)

WILUS, JEFFREY O. (MST-5)

SMITH, JAMES L. (MST-5)

$38-42$

PREPARATION AND CHARACTERIZATION OF

PLUTONIUM-240 OXIDE USED FOR

HALF - UFE MEASUREMENT.

INT. J. APPL. RADIAT. ISOT.,

V.35, P.159-61. 1984

REIN, JAMES E. (CMB-1)

WATERBURY, GLENN R. (CMB-1)

38-43

SELF - IRRADIATION EFFECTS IN 238 PLUTONIUM-SUBSTITUTED ZIRCONOLTE

I. TEMPERATURE-DEPENDENCE OF DAMAGE.

J. NUCL. MATER.

V.126, P.245-54. 1984.

CLINARD, FRANK W., JR. (MST-5)

PETERSON, DEAN E. (MST-5)

ROHR, DANA L (MST-5)

HOBBS, UNN W. (MST-5)

$38-44$

STRUCTURAL DAMAGE IN A SELF -

IRAADIATED ZIRCONOLITE - BASED CERAMIC.

TIC.

1983. 22P. MN (LA-UR-83-1473)

ALSO PUBLISHED IN:

NUCL. INSTRUM. METHODS,

V.B1, P.581-6. 1984.

CLINARD, FRANK W., JR. (MST-5)

ROHR, DANA L. (MST-5)

ROOF, RAYMOND BRADLEY, JR. (MST-5)

38-45

STUDY OF DEFECTS PRODUCED IN TUNGSTEN

BY 800 MEV PROTONS USING FIELD ION

MICROSCOPY.

J. NUCL MATER.

V.122-3, P.996-1001. 1984

FARNUM, DAVID J. (MP-13)

SOMMER, WALTER F., JR. (MP-13)

INAL, OSMANT.
$38-46$

SUPERCONDUCTING GROUND STATE OF A STRONGLY INTERACTING ELECTRON SYSTEM URANIUM BERYLLUM (13).

TIC,

1983. 8P. MN (LA-UR-83-2982)

OTT, HANS R.

RUDIGIER, $\mathrm{H}$.

FISK, ZACHARY (MST-5)

SMITH, JAMES L (MST-5)

$38-47$

TRANSITION TO DRAG-CONTROLLED DEFORMATION IN COPPER AT HIGH STRAIN RATES.

CONFERENCE ON THE MECHANICAL PROPERTIES OF MATERIALS AT HIGH RATES OF STRANS, 3RD, 1984, OXFORD.

PROC., P.71-80. INSTITUTE OF PHYSICS. 1984.

FOLLANSBEE, PAUL S. (MST-5)

REGAZZONI, GILLES (MST-5)

KOCKS, U. FRED (CMS)

$38-48$

VAPORIZATION THERMODYNAMICS AND

ENTHALPY OF FORMATION OF ALUMINUM SILICON CARBIDE.

J. AM. CERAM. SOC.,

V.67, P.575-8. 1984.

BEHRENS, ROBERT G. (CMB-3)

RINEHART, GARY H. (CMB-3)

$38-49$

$X$ - P - T PHASE DIAGRAM FOR THE

GAMMA - ALPHA TRANSITION IN

CERIUM $0.9-X$ LANTHANUM X THORIUM 0.1

ALLOYS.

PHYS. REV.

V.29B, P.4017-25. 1984.

LAWRENCE, J.M

THOMPSON, JOE D. (P-10)

FISK, ZACHARY (MST-5)

SMITH, JAMES L. (MST-5)

BATLOGG, $B$.

38-50

1.2 MBAR SHOCK LOADED 304 STANNESS

STEEL - RESIDUAL STRUCTURE AND

PROPERTIES.

SHOCK WAVES IN CONDENSED MATTER 1983, SANTA FE. PROC., P.419-21.

ELSEVIER, 1984.

STAUDHAMMER, KARL P. (MST-5)

JOHNSON, KAYE ALLAN (MST-11)

OLINCER, BARTON W. (M-6) 
39-1

A PRIORI LOCAL GRID REFINEMENT IN THE MULTIGRID METHOD.

ELLIPTIC PROBLEM SOLVERS II,

G. BIRKHOFF \& A. SCHOENSTADT, ED.,

P.439-51. ACADEMIC PRESS, INC., 1984.

(515.35/E46PR/1983)

ALSO PUBLISHED IN:

TIC.

1983. 14P. MN (LA-UR-83-0023)

DENDY, JOEL E., JR. (T-7)

39-2

ACQUISITION SYSTEMS FOR HEAT TRANSFER MEASUREMENT.

TIC,

1983. 32P. MN (LA-UR-83-3205)

DEWITT, RICHARD J. (WX-11)

39.3

ADAPTIVE MESH TECHNIQUES FOR FRONTS IN

STAR FORMATION.

INTL CONF. OF THE CENTER FOR

NONLINEAR STUDIES, 3RD, 1983,

LOS ALAMOS. PROC., P.408-25.

NORTH-HOLLAND PHYSICS PUBL, 1984. (530.15/161CE/3RD/1983)

WINKLER, KARL-HEINZ ADOLF (X-DOT

NORMAN, M. L. (X-8)

NEWMAN, MICHAEL J. (X-2)

$39-4$

AEROSOL MULTIPLE SCATTERING CODE ASCAT.

LOS ALAMOS;

1984. 50P. (LA-10046-MS)

ZARDECKI, ANDREW (T-DO)

GERSTL, S.A. W. (T-DOT)

$39-5$

AIRID - AN APPUICATION OF THE KAS/

PROSPECTOR EXPERT SYSTEM BUILDER TO

AIRPLANEIDENTIFICATION.

APPLICATIONS OF ARTIFICIAL

INTELLIGENCE, 1984, ARLINGTON.

PROC., P.73-9. SPIE, 1984.

(001.535/A652AR/1984)

ALSO PUBLISHED IN:

TIC.

1984. BP. MN (LA-UR-84-0988)

ALDRIDGE, JACK P. (NSP/AWT)

39-6

ALEORITHM FOR SINGULAR VALUE

DECOMPOSITION.

REAL TIME SIGNAL PROCESSING, $7 T H$,

1984, SAN DIEGO. PROC., P.22-9.

SPIE, 1984. (621.38/R327T/7TH/1984)

ROSS, O. C. (E-6)

\section{MATHEMATICS AND COMPUTERS}

39-7

ANALYSIS OF MATERIALS ACCOUNTING DATA.

MATHEMATICALAND STATISTICAL METHODS IN NUCLEAR SAFEGUARDS, F. ARGENTESI, ET AL., P.143-5. HARWOOD ACADEMIC PUBLISHERS, 1983. (621.483/M426ST) SHIPLEY, JAMES P., JR. (Q-DO/SG)

39-8

APPROXIMATE AND EXACT SCHEMES FOR

PHOTON-MAXWELLIAN ELECTRON CROSS SECTIONS.

COMPUT. PHYS. COMMUN.,

V.34, P.77-86. 1984

WIENKE, BRUCE R. (C-10)

LATHROP, BRAXTON L. (C-3)

$39-9$

ART AND THE COMPUTER.

ART AND THE COMPUTER. MCGRAW-HILL

BOOK CO. 1984. (760/P971AR)

PRUEITT, MELVIN L. (X-7)

39-10

ATTACKS ON COMPUTERS - CONGRESSIONAL

HEARINGS AND PENOING LEGISLATION.

SYMPOSIUM ON SECURITY AND PRIVACY,

1984, OAKLAND. PROC., P.180-6.

IEEE, 1984.

ALSO PUBLISHED IN:

TIC,

1984. 8P. MN (LA-UR-84-0650)

BAILEY, DAVID J. (OS-4)

39-11

BENCHMARKING UNSTAUCTURED SYSTEMS.

TIC,

1983. 13P. MN (LA-UR-83-1207)

BRICE, LINDA (ADP-2)

CONNELL, JOHN L. (ADP-2)

LOCKHART, MILTON GLENN (ADP-2)

39-12

BREATHER-KINK-ANTIKINK-PAIR

CONVERSION IN THE DRIVEN SINE-GORDON

SYSTEM.

PHYS. REV

V.29A, P.350-4. 1984

LOMDAHL, PETER S. (CNLS)

OLSEN, OLEH.

SAMUELSEN, MOGENS R.

39-13

CHARACTERIZING THE PARALLELISM IN

RULE - BASED EXPERT SYSTEMS.

HAWAII NTL. CONF. ON SYSTEM

SCIENCES, 18TH, 1985, HONOLULU.

PROC., P.47-54. THE CONF., 1985.

(620.7/H112S/1985N.2)

DOUGLASS, ROBERT J. (C-10)
39-14

CLASS OF EXACTLY SOLVABLE POTENTIALS

I - ONE - DIMENSIONAL SCHRODINGER

EQUATION.

ANN. PHYS.

V.152, P.203-18. 1984.

GINOCCHIO, JOSEPH N. (T-5)

39-15

COMPARISON OF FMS VERSUS TDMS APPLICATIONS.

TIC,

1983. 31P. MN (LA-UR-83-3340)

WERNER, NANCY E. (ADP-2)

39-16

COMPARISON OF METHODS FOR SOLVING NONLINEAR FINITE - ELEMENT EQUATIONS

IN HEAT TRANSFER.

CHEM. ENG. COMMUN.,

V.26, P.269-84. 1984.

JOHNSON, NORMAN LEE $(W X-4)$

GRAHAM, ALAN LYMAN (WX-4)

CORT, GEORGE E. $(W X-4)$

$39-17$

COMPUTER GRAPHICS SUPPORT SERVICE

TIC.

1984. 13P. MN (LA-UR-84-1120)

DICKMAN, DONALD O. (Q-6)

39-18

COMPUTING DIVISION TWO - YEAR

OPERATIONALPLAN

LOS ALAMOS

1984. PAGED IN SECTIONS (LA-09978-MS)

EWALD, ROBERT H. (C-DO)

39-19

CONJUGATE GRADIENT PRECONDITIONERS FOR

VECTOR AND PARALLEL PROCESSORS.

ELIIPTIC PROBLEM SOLVERS II,

G. BIRKHOFF \& A. SCHOENSTADT, ED.,

P.127-39. ACADEMIC PRESS, INC., 1984

(515.35/E46PR/1983)

ALSO PUBLISHED IN

TIC.

1983. 14P. MN (LA-UR-83-0272)

JORDAN, THOMAS LEE (C-3)

$39-20$

CONSERVATIVE REZONING (REMAPPING) FOR

GENERAL QUADRILATERAL MESHES.

J. COMPUT. PHYS.

V.54, P.411-24. 1984.

DUKOWICZ, JOHN K. (T-3) 
39-21

CONTINUED FRACTION EXPANSIONS FOR THE COMPLETE, INCOMPLETE, AND RELATIVISTIC

PLASMA DISPERSION FUNCTIONS.

J. MATH. PHYS.

V.25, P.466-8. 1984.

PERATT, ANTHONY L $(X-8)$

39-22

CON1D-A COMPUTER PROGRAM FOR

CALCULATING SPHERICALLY SYMMETRIC

DROPLET COMBUSTION.

LOS ALAMOS,

1884. 56P. (LA-10269-MS)

JANSSEN, ROBERT D. (CNLS)

O'ROURKE, PETER J. (T-3)

39-23

CRAY - 1 INSTRUCTION ANALYSIS - A

COMPARISON OF TWO METHODS.

TIC,

1983. 24P. MN (LA-UR-83-1500)

MARTIN, JOANNE L. (C-3) WARNOCK, TONYT.

39-24

CRAYNAX INTERACTIVE GRAPHICS SYSTEMS.

TIC,

1983. 4P. MN (LA-UR-63-3397)

WILLERTON, DONALD L (C-3)

39-25

CROSS - VALIDATION OF REGRESSION

MODELS.

J.AM.STAT. ASSOC.

V.79, P.575-83. 1984.

PICARD, RICHARD R. (S-1)

COOK, R. DENNIS (S-1)

39-26

CURRENT RESEARCH IN PARALLEL

MICROPROCESSING SYSTEMS AT LOS ALAMOS.

TIC,

1984. 6P. MN (LA-UR-64-1324)

ETHRIDGE, C. DWAYNE (C-5)

39-27

CURRENT STATUS OF LNK ACCESS CONTROL AND ENCRYPTION SYSTEM.

TIC,

1984. 5P. MN (LA-UR-84-0465) SPRINGER, EDWARD A. (OS-4)

39-28

CYCLOTOMIC IDENTITY.

CONTEMP. MATH.,

V.34, P.19-27. 1984

METROPOUS, NICHOLAS C. (T-DOT)

ROTA, GIAN-CARLO

$39-29$

DATABASE TRANSFERS BETWEEN SEVERAL

SYSTEMS.

TIC

1983. 10P. MN (LA-UR-83-3394)

DOLL, MARGARET A. (C-3)
39-30

DESIGN AND DESCRIPTIVE TOOLS FOR

SYSTOLIC ARCHITECTURES.

REAL TIME SIGNAL PROCESSING, $7 T H$,

1984, SAN DIEGO. PROC., P.67-74.

SPIE, 1984. (621.38/R32TT/7TH/1984)

LEWIS, PAUL S. (E-8)

39-31

DIELECTRIC POLARIZATION IN RANDOM

MEDIA.

J. STAT. PHYS.,

V.35, P.49-74. 1984.

RAMSHAW, JOHN D. (T-3)

39-32

DIFFUSION - SYNTHETIC ACCELERATION METHODS FOR DISCRETE-ORDINATES

PROBLEMS.

ADVANCES IN REACTOR COMPUTATIONS,

1983, SALT LAKE CITY. PROC.

P.705-24. AMER. NUCLEAR SOCIETY,

1983. (621.483/A244RE/1983/N.2)

ALSO PUBUSHED IN:

TC.

1983. 21P. MN (LA-UR-82-3581)

ALSO PUBLISHED IN:

TRANSP. THEORY STAT. PHYS.

V.13, P.107-26. 1984.

LARSEN, EDWARD W. (T-1)

39-33

DIGITAL FILTERING IN A DISEASE

DETECTION SYSTEM.

ताC.

1983. 15P. MN (LA-UR-63-3423)

BROWN, R. R. (E-5)

39-34

DOS21 - PCDOS, 2.0 SUPPORT SUBROUTINE

PACKAGE FOR THE IBM PERSONAL COMPUTER. LOS ALAMOS,

1984. 4P. (LA-10142-MS)

MC GIRT, A. FRANK (C-S)

$39-35$

DROPLETS OF 3 HELUM ATOMS.

NATO ADVANCED RESEARCH WORKSHOP ON

MONTE CARLO METHODS IN QUANTUM

PROBLEMS, 1982, PARIS. PROC., P.33-

9. D. REIDEL, 1984.

(530.12/N279WO/1982)

SCHMIDT, KEVIN E. (T-5)

39-36

DYNAMIC ACCELERATION OF NONLINEAR ITERATIONS.

ELLIPTIC PROBLEM SOLVERS $\|$,

G. BIRKHOFF \& A. SCHOENSTADT, ED.,

P.301-13. ACADEMIC PRESS, INC. 1984.

(515.35/E46PR/1983)

HYMAN, JAMES M. (T-7)

MANTEUFFEL, THOMAS A. (C-3)
39-37

EVENT84 USER'S MANUAL - A COMPUTER CODE FOR ANALYZING EXPLOSION - INDUCED

GAS - DYNAMIC TRANSIENTS IN FLOW

NETWORKS.

LOS ALAMOS

1984. 94P. (LA-10312-M)

MARTIN, RICHARD A. (Q-DO)

WILSON, TIMOTHYL (Q-6)

$39-38$

EZPIX - A TABLET ENTRY METHOD FOR COMPUTER GENERATED SUIDES, DRAWINGS,

AND GRAPHS.

LOS ALAMOS,

1984. 27P. (LA-10208-M)

WILLIAMS, JOEL M. (MST-6)

39-39

FAMILY OF ALMOST PERIODIC

SCHROEDINGER OPERATORS.

TIC,

1983. 27P. MN (LA-UR-83-2295)

BAKER, GEORGE A., JR. (T-11)

BESSIS, DANIEL

MOUSSA, PIERRE

39-40

FAST OPTIMAL ALIGNMENT.

NUCLEIC ACIDS RES.

V.12, P.175-9. 1984

FICKETT, JAMES WILDON (T-10)

39-41

FAST SCHEME FOR PHOTON - MAXWEULAN

ELECTRON CROSS SECTIONS.

J. COMPUT. PHYS.,

V.53, P.391-44. 1984

WIENKE, BRUCE R. (C-3)

LATHROP, BRAXTON L (C-3)

39-42

FLAME FRONTS WITH COMPLEX CHEMICAL NETWORKS.

TाC.

1983. 38P. MN (LA-UR-83-2886)

FIFE, PAUL C.

NICHOLS, BASIL (T-7)

$39-43$

FLAME FRONTS WITH COMPLEX CHEMICAL NETWORKS.

INTL. CONF. OF THE CENTER FOR

NONUNEAR STUDIES, 3RD, 1983,

LOS ALAMOS. PROC., P.182-97.

NORTH-HOLLAND PHYSICS PUBL, 1984.

(530.15/161CE/3RD/1983)

FIFE, PAUL C.

NICOLAENKO, BASIL (T-7/CNLS)

$39-44$

HARDWARE DESCRIPTION LANGUAGES FOR

SYSTOUC ARCHITECTURES.

LOS ALAMOS,

1984. 135P. (LA-10255-T)

LEWIS, PAUL S. (E-8) 
$39-45$

HGRAPH - A SUBROUTINE PACKAGE FOR THE IBM PERSONAL COMPUTER TO SUPPORT THE HERCULES GRAPHICS CARD.

LOS ALAMOS,

1984. 10P. (LA-10144-MS)

MC GIRT, A. FRANK (C-9)

$39-46$

HIGHLY PARALLEL COMPUTING.

IDENTIFYING RESEARCH AREAS IN THE

COMPUTER INDUSTRY TO 1995, R.F.

COTELLESSA, ED., P.74-82. NOYES PUBL.

1984. (001.64/119RE/1983)

BROWNE, JAMES C.

ARDEN, BRUCE

ARVIND,

BASKETT, FOREST (C-11)

BUZBEE, BILLYL. (C-DO)

FRANKLIN, MARK

KELLER, ROBERT M.

KUNG, H. T.

LAWRIE, DUNCAN

RIS, FRED.

SCHORR, HERBERT

SIEGEL, HOWARD J.

SNYDER, LAWRENCE

VOIGT, ROBERT

39-47

HOW TO TALK TO YOUR COMPUTER

LTTERALLY.

TIC.

1983. 8P. MN (LA-UR-83-3393)

FORD, WENDELL (Q-4)

39-48

MPLAS - MULTIGROUP CROSS SECTION AND REACTION RATE PROCESSOR FOR COUPLED HYDROGEN, HYDROGEN AND HYDROGEN PLUS TRANSPORT APPLICATIONS IN PLASMAS.

COMPUT. PHYS. COMMUN.,

V.34, P.87-99. 1984.

WIENKE, BRUCE R. (C-3)

MOREL, J. E. (X-6)

CAYTON, THOMAS E. (CTR-6)

HOWELL, ROBERT B. (CTR-2)

39-49

ILLUSTRATOR'S VIEW OF MORE EFFECTIVE TECHNICAL COMMUNICATION THROUGH COMPUTER GRAPHICS.

TIC.

1981. 12P. MN (LA-UR-80-3457)

DE GASPERI, KATHLEEN D. (H-1)

39-50

IMPORTANCE ESTIMATION IN FORWARD

MONTE CARLO CALCULATIONS.

NUCL. TECHNOL./FUSION,

V.5, P.90-100. 1984.

BOOTH, THOMAS E. $(X-6)$

HENDRICKS, JOHN S. $(X-6)$
39-51

INFORM SYSTEM TWO - YEAR PLAN

FY 1984 - 1985.

LOS ALAMOS,

1984. PAGED IN SECTIONS.

(LA-10185-MS)

THORN, ROBERT N. (DIR-O)

39-52

INTERACTIVE BENCHMARK COMPARISON

BETWEEN A VAX 11/780 AND A VAX 11/782.

TIC,

1983. 5P. MN (LA-UR-83-3396)

LUBECK, OLAF M. (C-3)

MARTINEZ, RONALD G. (C-3)

39-53

INTERFACING CAMAC TO A HIGH - SPEED

MULTIPROCESSOR BIT SLICE DATA-

ACQUISTION SYSTEM.

TIC.

1984. 8P. MN (LA-UR-84-1047) GEISIK, CARL (AT-2)

39-54

INTERNALLY CONTRACTED MCSCF-SCEP

CALCULATIONS.

ADVANCED THEORIES AND COMPUTATIONAL

APPROACHES TO THE ELECTRONIC

STRUCTURE OF MOLECULES, C. E.

DYKSTRA, ED., P.79-103. D. REIDEL,

1984.

WERNER, HANS-JOACHIM (T-12)

REINSCH, ERNST-ALBRECHT

39-55

INVARIANCE PROPERTIES IN HERMITE - PADE

APPROXIMATION THEORY.

J. COMPUT. APPL. MATH.

V.11, P.49-55. 1984.

BAKER, GEORGE A., JR. (T-11)

39-66

INVENTORY VERIFICATION USING THE

INSPECTOR'S SUFFICIENT STATISTICS. MATHEMATICAL AND STATISTICAL METHODS IN NUCLEAR SAFEGUARDS, F. ARGENTESI,

ET AL, P.351-83. HARWOOD ACADEMIC

PUBLISHERS, 1983. (621.483/M426ST)

SHIPLEY, JAMES P., JR. (Q-DO/SG)

39-57

INVESTIGATION OF AN EMPIRICAL

PROBABILITY MEASURE BASED TEST

FOR MULTIVARIATE NORMALTTY.

TIC,

1984. 33P. MN (LA-UR-84-1499)

BOOKER, JANE M. (S-1)

JOHNSON, MARK E. (S-1)

BECKMAN, RICHARD J. (S-1)
$39-58$

KRAK - A COMPUTER PROGRAM FOR TWO PHASE, TWO-COMPONENT POROUS FLOW ANDFRACTURE

LOS ALAMOS.

1984. 95P. (LA-09703)

TRAVIS, BRYAN J. (ESS-5)

DAVIS, ALVIN H. (CMB-9)

KUNKLE, THOMAS D. (ESS-5)

DEUPREE, ROBERT G. (ESS-5)

39-59

LAMPF NUCLEAR CHEMISTRY DATA

ACQUISTTION SYSTEM.

DIGITAL EQUIPMENT COMPUTER USERS

SOCIETY. SYMPOSIUM, 1983, LAS VEGAS.

PROC., P.251-8. DECUS, 1983.

ALSO PUBLISHED IN:

TIC,

1983. 10P. MN (LA-UR-63-3410)

GIESLER, GREGG CARL (CNC-11)

$39-60$

LEARNING ABOUT SUPERCOMPUTERS ON A MICROCOMPUTER WITH NO KEYBOARD - A SCIENCE MUSEUM EXHIBIT.

TIC.

1984. 9P. MN (LA-UR-83-2885)

STODDARD, MARYL (C-10)

BUZBEE, BILLYL (C-DO)

39-61

LNEAR CHARACTERISTIC - NODAL TRANSPORT

METHOD FOR THE TWO-DIMENSIONAL $(X, Y)$ -

GEOMETRY MULTIGROUP DISCRETE ORDINATES

EQUATIONS OVER ARBITRARY TRIANGLE MESH.

LOS ALAMOS

1984. 127P. (LA-10092-T)

PATERNOSTER, RICHARD R. (X-7)

39-62

UNEAR TRANSPORT IN NONHOMOGENEOUS

MEDIA III.

J. MATH. PHYS.

V.25,P.1511-6. 1984

DANIEL, $R$.

POMRANING, GERALD C. $(X-7)$

LARSEN, EDWARD W. $(x-6)$

39-63

LINEAR TRANSPORT IN NONHOMOGENEOUS MEDIA.

TRANSP. THEORY STAT. PHYS.

V.13, P.511-9. 1984.

LARSEN, EDWARD W. $(X-6)$

39-64

LINER PACKAGE - AN INCIPIENT MHD

CAPABILITY FOR CCUBE.

LOSALAMOS,

1984. 6P. (LA-09969-MS)

GISLER, GALEN $(X-B)$

FAEHL, R. J. $(X-8)$ 
39-65

LONG RANGE PLANNING COMMITTEE FOR LAMPF

COMPUTING NEEDS REPORT.

LOS ALAMOS

1984. 29P. (LA-10103-MS)

HOEHN, MARTHA V. (MP-13)

AMANN, JAMES F. (MP-10)

BUTLER, HAROLO S. (MP-1)

DAWSON, W. K. (MP-1)

HOFFMAN, EARL WAYNE (MP-1)

HOFTEIZER, J.

KALETKA, $M$.

KINNISON, W. WAYNE (P-DO)

KOZLOWSKI, THOMAS (MP-10)

MC NAUGHTON, MICHAEL W. (MP-13)

MISCHKE, RICHARD E. (MP-4)

OOTHOUDT, M. A. (MP-1)

POORE, RAY V. (P-9)

SHERA, E. BROOKS (P-3)

TURPIN, S. E.

WOUTERS, JAN M. (INC-11)

$39-66$

LONG-RANGE PERCOLATION IN ONE

DIMENSION.

J. PHYS. A

V.17, P.2349-50. 1984.

SPOUGE, JOHN L $(\mathrm{T}-10)$

39-67

LOS ALAMOS NATIONAL LABORATORY COMPUTER

BENCHMARKING, 1983.

LOS ALAMOS,

1984. 15P. (LA-10151-MS)

GRIFFIN, JAMES HARPER (C-10)

SIMMONS, MARGARET L. (C-10)

$39-68$

LOS ALAMOS REAL-TIME AND DIGITAL

RADIOGRAPHY PLANS.

TIC.

1983. 4P. MN (LA-UR-83-3477)

STRONG, RONALD DEAN $(W \times-3)$

$39-69$

MECA - A SUPERCOMPUTER FOR MONTE CARLO.

LOS ALAMOS.

1984. 5P. (LA-10005)

SOLEM, JOHNDALE C. (T-DO)

$39-70$

MODELING THE USER IN INTELLIGENT

USER INTERFACES.

TIC.

1983. 9P. MN (LA-UR-83-1392)

STODDARD, MARY L (C-10)

DOUGLASS, ROBERT J. (C-10)

39-71

MONTE - CARLO METHOD FOR QUANTUM

BOLTZMANN STATISTICAL MECHANICS.

J. CHEM. PHYS.,

V.80, P.2239-40. 1984.

DOLL, JIMMIE D. (CHM-2)

FREEMAN, DAVID L. (CHM-2)
39-72

MONTE CARLO METHOD FOR QUANTUM

BOLTZMANN STATISTICAL MECHANICS

USING FOURIER REPRESENTATIONS OF

PATH INTEGRALS.

J. CHEM. PHYS.,

V.80, P.5709-18. 1984.

FREEMAN, DAVOL (CHM-2)

DOL, JIMMIE D. (CHM-2)

39-73

MONTE CARLO SAMPLNG OF COMPTON PHOTON

SCATTERING OFF MOVING ELECTRONS.

TRANS. AM. NUCL SOC.

V.47, P.226-8. 1984.

HENDRICKS, JOHN S. (X-6)

BOOTH, THOMAS E. $(\alpha-6)$

$39-74$

MONTE CARLO VARIANCE COMPARISON FOR

EXPECTED VALUE VERSUS SAMPLED

SPLITING.

TRANS. AM. NUCL SOC.

V.46, P.433. 1984.

BOOTH, THOMAS E. $(\alpha-6)$

39.75

MSS GENERIC MODEL - A BEGINNING.

TKC.

1984. 8P. MN (LA-UR-84-0139)

COLLINS, MAURICE W. (C-8)

MILLER, STEPHEN

39-76

MULTDDOMAIN, MULTIRECORD - TYPE

DATATRIEVE - 11 DATABASES.

TIC,

1983. 7P. MN (LA-UR-83-2980)

HORNING, R. R. (C-4)

39-77

NECESSARY AND SUFFICIENT CONDITIONS

FOR THE EXISTENCE OF A CONJUGATE

GRADIENT METHOD.

SIAM J. NUMER. ANAL.

V.21,P.352-62. 1984.

FABER, VANCE (C-3)

MANTEUFFEL, THOMAS (C-3)

39-78

NETWORK FILE STORAGE SYSTEM.

TIC.

1983. 10P. MN (LA-UR-83-2678)

COLLINS, MAURICE W. (C-8)

DEVANEY, MARJORIE A. (C-8)

WLLBANKS, EMILY W. (C-B)

$39-79$

NUMERICAL METHODS FOR TRACKING

INTERFACES.

INTL CONF. OF THE CENTER FOR

NONLINEAR STUDIES, 3RD, 1983

LOS ALAMOS. PROC., P.396-407.

NORTH-HOLLAND PHYSICS PUBL, 1984.

(530.15/161CE/3RO/1983)

ALSO PUBLSHED IN:

TIC,

1983. 26P. MN (LA-UR-83-3049)

HYMAN, JAMES M. (T-7)
39-80

NUMERICAL METHODS FOR TRACKING

INTERFACES.

LOS ALAMOS,

1984. 20P. (LA-09917-MS)

HYMAN, JAMES M. (T-7)

39-81

ON THE REACTION ZONE THICKNESS OF

PREMIXED LAMINAR FLAMES.

COMBUST. SCI. TECHNOL.

V.38, P.337-43. 1884

O'ROURKE, PETER J. (T-3)

STEIN, LELAND R. (T-3)

$39-82$

ONE - DIMENSIONAL MODEL FOR THREE-

DIMENSIONAL TRANSPORT IN A PIPE

TRANSP. THEORY STAT. PHYS.

V.13, P.599-614. 1984.

LARSEN, EDWARD W. $(\alpha-6)$

39-83

ONE-DIMENSIONAL APPROXIMATIONS FOR A

QUADRATIC IKEDA MAP.

PHYS. LETT.

V.104A, P.69-62. 1984.

CARR, J.

EILBECK, J. CHRIS (CNLS)

$39-84$

OPTIMAL SMOOTHING OF SITE - ENERGY

DISTRIBUTIONS FROM ADSORPTION ISOTHERMS.

TIC.

1983. 11P. MN (LA-UR-83-2848)

BROWN, LEE F. (ESS-5)

TRAVS, BRYAN J. (ESS-5)

39-85

PARALLEL ALGORTTHMS.

HAWAII INTL CONF. ON SYSTEM

SCIENCES, 18TH, 1985, HONOLULU.

PROC., P.1. THE CONF., 1985.

(620.7/H112S/1985N.2)

ANDERSON, JAMES WAYNE (C-8)

39-86

PERCEPTION OF GRAPHIC SYSTEM DATA BASE

PROBLEMS AND NEEDS FROM A NUMERICAL

CONTROL PROGRAMMER'S VIEWPOINT.

nC.

1984. 21P. MN (LA-UR-84-1526)

MAIER, OTTO A. (MEC-3)

39-87

PAEDICTING LOW - PROBABILTY /HIGH -

CONSEQUENCE EVENTS.

LOW-PROBABILTY/HIGH-CONSEQUENCE

RISK ANALYSIS.

ISSUES, METHODS, AND CASE STUDIES,

R.A. WALLER, V.T. COVELLO, EDS.

P.187-97. PLENUM, 1984.

ALSO PUBLSHED IN.

TIC.

1982. 38P. MN (LA-UR-82-1668)

MARTZ, HARAY F., JR. (S-1)

BRYSON, MAUAICE C. (S-1) 
$99-88$

PRELIMINARY USER'S GUIDE FOR BICONE

ON THE CRAY.

LOS ALAMOS.

1984. 46P. (LA-10183-M)

SNELL, CHARLES M. $(X-8)$

KWAN, THOMAS J. T. $(X-8)$

39-89

PROCESSING TIME USING DATATRIEVE - 11 ,

CLUNKS, AND FORTRAN.

TIC.

1983. 6P. MN (LA-UR-63-2979)

HORNING, R. R. (C-4)

GOODE, WILLIAM R.

39-90

PSEUDO - RANDOM TREES IN MONTE CARLO.

PARALLEL COMPUT.,

V.1, P.175-80. 1984

FREDERICKSON, PAUL O. (C-3)

HIROMOTO, ROBERT E. (C-3)

JORDAN, THOMAS LEE (C-3)

SMITH, BURTON

WARNOCK, TONYT

39-91

OKPLT USERS GUIDE, VERSION 2.0

LOS ALAMOS

1984. 33P. (LA-10094-M)

BROWNING, RICHARDV. (WX-11)

OAKES, WILLIAM R. (WX-11)

WINKLER, CAROLYN M. $(W X-11)$

$39-92$

RADIATION TRANSFER IN VEGETATIVE

CANOPIES AND OTHER LAYERED MEDIA-

RAPIDLY SOLVABLE EXACT INTEGRAL EQUATION

NOT REOUIRING FOURIER RESOLUTION.

J. QUANT. SPECTROSC. RADIAT. TRANSFER

V.31,P.71-82. 1984

GUTSCHICK, VINCENT P. (LS-6)

WIEGEL; FREDERIK $W$.

39-93

RECOVER IT YOURSELF WITH USER LOGGING

TIC.

1984. 11P. MN (LA-UR-83-3290)

WEIR, DIANE (ADP-2)

$39-84$

REMARKS ON THE KURAMOTO - SIVASHINSKY

EQUATION.

INTL CONF. OF THE CENTER FOR

NONLINEAR STUDIES, 3RD, 1983,

LOS ALAMOS. PROC., P.391-5.

NORTH-HOLLAND PHYSICS PUBL, 1984.

(630.15/161CE/3AD/1983)

ALSO PUBLISHED IN

TIC.

1983. 9P. MN (LA-UR-83-2862)

NICOLAENKO, BASIL (T-7/CNLS)

SCHEURER, BRUNO (CNLS)

$39-95$

REMOTE - MODEM COMMUNICATOR PROGRAM

FOR THE IBM PEASONAL COMPUTER.

LOS ALAMOS,

1984. 17P. (LA-10143-MS)

MC GIRT, A. FRANK (C-9)
$39-96$

REVIEW OF THE ANSI METAFILE AND

VIRTUAL DEVICE INTERFACE STANDARDIZATION

ACTIVITES.

TIC,

1983. 9P. MN (LA-UR-83-2257)

REED, THEODORE N. (C-6)

$39-97$

RFO SIMULATION COOE.

LINEAR ACCELERATOR CONFERENCE,

1984, SEEHEIM. PROC., P.327-9.

1884. (GSI-84-11)

LYSENKO, WALTER P. (AT-6)

$39-98$

SAMPLE - SOFTWARE FOR VAX FORTRAN

EXECUTION TIMING.

TKC.

1983. 4P. MN (LA-UR-83-3421)

LOWE, LEWIS H. $(W X-11)$

39.99

SATELLITE GRAPHICS OUTPUT STATION.

TIC.

1981. 18P. MN (LA-UR-80-3716)

WOLF, RICHARD J. (C-6)

39-100

SEMIGROUP ASSOCIATED WITH A QUASI-

LNEAR SYSTEM IN WHICH THE COUPLING IS

UNEAR.

TIC,

1983. 19P. MN (LA-UR-83-3545)

PIMBLEY, GEORGE H. (T-14)

39-101

SEQUENTIAL UKELIHOOD-RATIO TESTS

APPLED TO SERIES OF MATERIALS

BALANCES.

MATHEMATICAL AND STATISTICAL METHODS

IN NUCLEAR SAFEGUARDS, F. ARGENTESI,

ET. AL., P.183-212. HARWOOD ACADEMIC

PUBLSHERS, 1983.(621.483/M426ST)

SHIPLEY, JAMES P.. JR. (Q-DO/SG)

39-102

SIMPLIFIED ADAPTIVE MESH TECHNIQUE

DERIVED FROM THE MOVNG FINITE ELEMENT

METHOD.

J. COMPUT. PHYS.

V.56, P.324-42. 1984

DUKOWICZ, JOHNK. (T-3)

39-103

SMILE USERS GUIDE - AMACRO

PREPROCESSOR FOR EXTENDING FORTRAN.

LOS ALAMOS

1984. 19P. (LA-09862-M)

LOWE, LEWIS H. (WX-11)

39-104

SOLUTION OF ELLIPTIC EQUATIONS USING

FAST POISSON SOLVERS.

J. COMPUT. PHYS.,

V.53, P.382-94. 1984

BERNHARDT, PAUL A. (ESS-7)

BRACKBILL, JEREMIAHU. $\mathbf{X - 1}$
39-105

SOLUTION OF MULTIDIMENSIONAL INVERSE

TRANSPORT PROBLEMS.

J. MATH. PHYS.,

V.25, P.131-5. 1984.

LARSEN, EDWARD W. $(X-6)$

39-106

SOLVNG CHEMICAL EQUILBRIUM PROBLEMS

USING NONLNEAR OPTIMIZATION

LOS ALAMOS

1984. 40P. (LA-10116-MS)

PARKINSON, WILLIAM J. (WX-4)

SANDERSON, JAMES G., JR. (X-7)

39-107

SOLVING VERY LARGE ELLPTIC PROBLEMS

ON A SUPERCOMPUTER WITH SOLID STATE

DISK.

J. COMPUT. PHYS.

V.55, P.340-5. 1984.

BUCHER, NGRID Y. (C-3)

JORDAN, THOMAS LEE (C-3)

39-108

SPECIAL FUNCTIONS AND GROUP THEORY IN THEORETICAL PHYSICS.

SPECIAL FUNCTIONS - GROUP

THEORETICAL ASPECTS AND APPUICATIONS

R.A. ASKEY, T.H. KOORNWINDER AND

W. SCHEMPP, ED., P.129-62. D. REIDEL

PUBL, 1984. (515.8/S741FU)

ALSO PUBUSHED IN:

nic,

1883. 36P. MN (LA-UR-83-2162)

BIEDENHARN, LAWRENCE C

GUSTAFSON, R. A.

LOHE, MAX A

LOUCK, JAMES D. (T-7)

MILNE, S. C.

$39-109$

STABILITY OF PLANAR MULTIFLUID PLASMA

EQUILIBAIA BY ARNOLD'S METHOD.

TIC,

1983. 27P. MN (LA-UR-83-3523)

HOLM, DARRYL D. (CNLS)

39-110

STATE SPACE METHOD OF FAULT TREE

ANALYSIS WITH APPLICATIONS.

LOS ALAMOS

1884. 186P. (LA-10298-T)

BARTHOLOMEW, ROBERT J. (Q-12)

39-111

SUPERSPACE APPROACH TO LATTICE

SUPERSYMMETRY.

J.MATH. PHYS.

V.25, P.2744-7. 1984.

KOSTELECKY, VACLAV ALAN (T-8)

RABIN, JEFFREY $M$.

39-112

TRACE - AN INTERACTIVE BEAM - TRANSPORT CODE.

LINEAR ACCELERATOR CONFERENCE,

1984, SEEHEIM. PROC., P.371-3.

1984. (GS1-84-11)

CRANDALL, KENNETHR. (AT-1)

RUSTHOI, DANIEL P. (AT-2) 
39-113

TRACR3D - A MODEL OF FLOW AND TRANSPORT IN POROUS/FRACTURED MEDIA.

LOS ALAMOS.

1984. 190P. (LA-09667-MS)

TRAVIS, BRYAN J. (ESS-5)

$39-114$

TRANIO - A SUBROUTINE PACKAGE FOR THE IBM PERSONAL COMPUTER TO SUPPORT THE TRANSIAC 6002 CAMAC CRATE CONTROLLER.

LOS ALAMOS,

1984. 13P. (LA-10145-MS)

MC GIRT, A. FRANK (C-9)

39-115

TRANSX - CTR, A CODE FOR INTERFACING MATXS CROSS-SECTION LIBRARIES TO NUCLEAR TRANSPORT CODES FOR FUSION SYSTEMS ANALYSIS.

LOS ALAMOS,

1984. 96P. (LA-09863-MS)

MAC FARLANE, ROBERT E. (T-2)

39-116

TVENT1P USERS MANUAL, A COMPUTER CODE FOR ANALYZING TORNADO - INDUCED GASDYNAMIC TRANSIENTS IN FLOW NETWORKS

LOS ALAMOS,

1984. 148P. (LA-10237-MS)

ANDRAE, RICHARD W. (Q-6)

TANG, P. K. $(X-7)$

GREGORY, WLLLAM S. (Q-6)

39-117

U - THE UNIVERSAL UTILITY CONTROLLER, REVISION 1.

LOS ALAMOS,

1984. 44P. (LA-09701-M, REV. 1)

SNELL, CHARLES M. $(X-8)$

$39-118$

USER'S GUIDE FOR TWODANT - A CODE

PACKAGE FOR TWO - DIMENSIONAL,

DIFFUSION - ACCELERATED, NEUTRAL-

PARTICLE, TRANSPORT.

LOS ALAMOS.

1984. 41P. (LA-10049-M)

ALCOUFFE, RAYMOND E. $(X-6)$

BRINKLEY, F. W., JR. $(X-6)$

MARR, DUANE R. $(x-6)$

O'DELL, R. DOUGLAS $(\alpha-6)$

$39-119$

USER'S GUIDE FOR TWODANT - A CODE

PACKAGE FOR TWO - DIMENSIONAL,

DIFFUSION - ACCELERATED, NEUTRAL-

PARTICLE TRANSPORT, REV. 1.

LOS ALAMOS.

1984. PAGED IN SECTIONS.

(LA-10049-M, REV. 1)

ALCOUFFE, RAYMOND E. $(X-6)$

BRINKLEY, F. W., JR. $(X-6)$

MARR, DUANE R. $(x-6)$

O'DELL, R: DOUGLAS $(X-6)$
39-120

USER'S GUIDE FOR TWOHEX - A CODE

PACKAGE FOR TWO - DIMENSIONAL, NEUTRAL-

PARTICLE. TRANSPORT IN EQUILATERAL

TRIANGULAR MESHES.

LOS ALAMOS,

1984. PAGED IN SECTIONS. (LA-10258-M)

WALTERS, WALLACE F. $(X-6)$

BRINKLEY, F. W., JR. $(X-6)$

MARR, DUANE R. (X-6)

39-121

USER'S GUIDE TO SENSITIVITY TESTING

ON COMPUTER MODELS AT LOS ALAMOS

NATIONAL LABORATORY.

LOS ALAMOS,

1984. 73P. (LA-10104-M)

WILLIAMS, DONNA S. (S-2)

39-122

USER'S MANUAL FOR GRIZZLY.

LOS ALAMOS,

1984.78P. (LA-10244-M)

ABDALLAH, JOSEPH (T-4)

39-123

USING INGRES AS A RAPID PROTOTYPING

DEVICE DURING DEVELOPMENT OF MANAGEMENT INFORMATION APPLICATIONS.

TIC.

1983. 24P. MN (LA-UR-63-1893)

BRICE, LINDA (ADP-2)

CONNELL, JOHN L (ADP-2)

SHAFER, DONALD F. (ADP-2)

$39-124$

VARIATIONAL METHOD FOR ADAPTIVE GRID

GENERATION.

TIC,

1983. 11P. MN (LA-UR-83-2493)

BRACKBILL, JEREMIAH U. $(X-1)$

$39-125$

VARIATIONS IN TASK AND THE IDEAL

OBSERVER.

APPUCATION OF OPTICAL

INSTRUMENTATION IN MEDICINE XI,

1983, ATLANTA, PROC., P. 60-7,

SPIE, 1983. (616.075/A652O/1983)

ALSO PUBUSHED IN:

TIC,

1983. 9P. MN (LA-UR-83-2166)

HANSON, KENNETH M. (M-4)

39-126

VECTORIZATION OF ALGORITHMS FOR SOLVING

SYSTEMS OF ELLIPTIC DIFFERENCE

EQUATIONS.

AMERICAN SOCIETY OF MECHANICAL

ENGINEERS. WINTER MEETING, 1983,

BOSTON. PROC., P.81-6. ASME, 1983.

ALSO PUBLISHED IN:

TIC,

1983. 9P. MN (LA-UR-83-1761)

BUZBEE, BILLYL. (C-DO)
39-127

VISAR - THINGS YOU SHOULD KNOW.

HIGH SPEED PHOTOGRAPHY, VIDEOGRAPHY, AND PHOTONICS, 1983, SAN DIEGO.

PROC., P.144-8. SPIE, 1983.

(778.37/H638SP/1983)

ALSO PUBLISHED IN:

TIC,

1983. 6P. MN (LA-UR-83-2291)

HEMSING, WILLARD F. (M-7)

39-128

VISAR - $21 / 2$ MINUTES FOR DATA

REDUCTION.

HIGH SPEED PHOTOGRAPHY, VIDEOGRAPHY, AND PHOTONICS, 1983, SAN DIEGO.

PROC., P.199-202. SPIE, 1983.

(778.37/H638SP/1983)

ALSO PUBLISHED IN:

TIC.

1983. 5P. MN (LA-UR-83-2290)

HEMSING, WILLARD F. (M-7)

39-129

VORT - A COMPUTER CODE FOR BUBBLY

TWO - PHASE FLOW

LOS ALAMOS,

1984. 94P. (LA-10021-MS)

COOK, THOMAS L. (ESS-5)

HARLOW, FRANCIS H. (T-3)

39-130

ZTL - A SUBROUTINE PACKAGE FOR THE IBM PERSONAL COMPUTER TO SUPPORT THE ZIATECH ZT1488 IEEE - 488 CONTROLLER.

LOS ALAMOS,

1984. 17P. (LA-10141-MS)

MC GIRT, A. FRANK (C-9)

MOORE, KURT R. (ESS-10)

39-131

ZTLCK - A SUBROUTINE PACKAGE FOR THE IBM PERSONAL COMPUTER TO SUPPORT THE ZIATECH ZT1488 REAL TIME CLOCK.

LOS ALAMOS.

1984. 8P. (LA-10146-MS)

MC GIRT, A. FRANK (C-9)

39-132

6TH BIENNIAL CUBE (COMPUTER USE BY

ENGINEERS) SYMPOSIUM.

OCTOBER 10-12, 1984.

LOS ALAMOS,

1984. 114P. (LA-10240-C)

STRAIGHT, J. W. (WX-8) 


\section{NUCLEAR PROPULSION SYSTEMS AND AEROSPACE SAFETY}

40-1

EXPERIMENTAL INVESTIGATIONS OF 238 PLUTONIUM BEHAVIOR IN SOIL COLUMNS.

LOS ALAMOS,

1984. 16P. (LA-10096-MS)

NELSON, G. B.

MERRERA, BENJIMEN (ENG-4)

STALNAKER, NELSON D. (CHM-1)

COFFELT, K.P. (CHM-1)

PATTERSON, JAMES H. (CHM-1)

HEATON, RICHARD C. (CHM-1)

MATLACK, GEORGE M. (CHM-1)

40-2

GENERAL - PURPOSE HEAT SOURCE

DEVELOPMENT - SAFETY TEST PROGRAM POSTIMPACT EVALUATION, DESIGN

ITERATION TEST 1.

LOS ALAMOS,

1984. 67P. (LA-09680-SR)

SCHONFELD, FRED W. (CMB-5)

40.3

GENERAL - PURPOSE HEAT SOURCE

DEVELOPMENT, SAFETY TEST PROGRAM, POSTIMPACT EVALUATION, DESIGN

ITERATION TEST 2

LOSALAMOS

1984. 49P. (LA-10012-SR)

SCHONFELD, FRED W. (MST-5)

GEORGE, TIMOTHY G. (MST-6)

40-4

GENERAL - PURPOSE HEAT SOURCE

DEVELOPMENT - SAFETY TEST PROGRAM

POSTIMPACT EVALUATION, DESIGN

ITERATION TEST 3.

LOS ALAMOS.

1984. 42P. (LA-10034-SR)

SCHONFELD, FRED W. (MST-5)

GEORGE, TIMOTHY G. (MST-5)

40.5

GENERAL - PURPOSE HEAT SOURCE DEVELOPMENT - SAFETY TEST PROGRAM - POST IMPACT EVALUATION, DESIGN ITERATION TEST 4.

LOS ALAMOS,

1984. 28P. (LA-10217-SA)

GEORGE, TIMOTHY G. (MST-5)

SCHONFELD, FRED W. (MST-5)
40-6

GENERAL - PURPOSE HEAT SOURCE DEVELOPMENT - SAFETY TEST PROGRAM POST IMPACT EVALUATION, DESIGN

ITERATION TEST 5.

LOS ALAMOS,

1984. 34P. (LA-10232-SR)

GEORGE, TIMOTHY G. (MST-5)

SCHONFELD, FRED W. (MST-5).

40-7

HEAT PIPE TECHNOLOGY ISSUES.

TIC,

1984. 29P. MN (LA-UR-84-1238)

MERRIGAN, MICHAEL A. (Q-13)

40-8

LONG - TERM EXPOSURE OF PRESSED

PLUTONIUM OXIDE HEAT SOURCES TO

AOUATIC ENVIRONMENTS.

LOS ALAMOS,

1984. 13P. (LA-10197-MS)

HEATON, RICHARD C. (CHM-1)

PATTERSON, JAMES H. (CHM-1)

KOSIEWICZ, STANLEY T. (CHM-1)

MATLACK, GEORGE M. (CHM-1)

STEINKRUGER, FREDERICK J. (INC-3)

NELSON, GILBERT B. (CMB-1)

VANDERBORGH, NICHOLAS E. (E-11)

COFFELT, K.P. (CHM-1)

HERRERA, BENJIMEN (ENG-4)

40-9

MATERIALS REQUIREMENTS FOR THE SP - 100

PROGRAM.

TRANS. AM. NUCL. SOC.,

V.46, P.331. 1984.

HANSON, J. E.

40-10

NUCLEAR SPACE POWER SYSTEMS FOR ORBIT

RAISING AND MANEUVERING.

ORBIT-AAISING AND MANEUVERING PROPULSION - RESEARCH STATUS AND NEEDS, L.H. CAVENY, ED., P.425-59. AMER. INST. OF AERONAUTICS AND

ASTRONAUTICS, INC., (629.1/064RA) BUDEN, DAVID (DAD/NP)

SULLIVAN, J. A (DAD/NP)
40-11

SPACE NUCLEAR SAFETY PROGRAM

JANUARY 1984.

LOS ALAMOS

1984. 23P. (LA-10162-PR)

BRONISZ, STANLEYE. (MST-5)

40-12

SPACE NUCLEAR SAFETY PROGRAM, AUGUST 1983.

LOS ALAMOS

1984. 7P. (LA-09980-PR)

BRONISZ, STANLEY E. (MST-5)

40-13

SPACE NUCLEAR SAFETY PROGRAM, SEPTEMBER 1983.

LOS ALAMOS,

1984. 10P. (LA-10000-PR)

BRONISZ, STANLEY E. (MST-5)

40-14

SPACE NUCLEAR SAFETY PROGRAM, NOVEMBER 1983.

LOS ALAMOS,

1984. 39P. (LA-10126-PR)

BRONISZ, STANLEYE. (MST-5)

40-15

SPACE NUCLEAR SAFETY PROGRAM,

DECEMBER 1983.

LOS ALAMOS,

1984. 16P. (LA-10134-PR)

BRONISZ, STANLEY E. (MST-5)

40-16

SPACE NUCLEAR SAFETY PROGRAM,

FEBRUARY 1984.

LOS ALAMOS.

1984. 24P. (LA-10190-PR)

BRONISZ, STANLEY E. (MST-5)

40-17

SPACE REACTOR FUELS PERFORMANCE AND

DEVELOPMENT ISSUES.

TIC.

1984. 7P. MN (LA-UR-64-1381)

WEWERKA, EUGENE M. (MST-DO) 


\section{NUCLEAR WASTE MANAGEMENT}

42-1

\author{
AMERICIUM THERMODYNAMIC DATA FOR THE \\ EQ3/6 DATABASE \\ LOS ALAMOS. \\ 1984. 39P. (LA-10040-MS) \\ KERRISK, JERRY F. (WX-4) \\ 42-2 \\ CESIUM AS AN ACTIVATABLE TRACER FOR \\ STUDYING BIOLOGICAL INTRUSION OF PLANT \\ ROOTS INTO WASTE BURIAL AREAS. \\ J. RADIOANAL. NUCL CHEM. \\ V.84, P.423-9. 1984. \\ GLADNEY, E. S. (H-8) \\ HAKONSON, THOMAS E. (LS-6) \\ MULLER, MARCIA (H-8)
}

42-3

DISTRIBUTION OF RADIONUCUIDES AND WATER

IN BANDEUER TUFF BENEATH A FORMER LOS

ALAMOS LIQUID WASTE DISPOSAL SITE AFTER

33 YEARS.

LOS ALAMOS,

1984. 51P. (LA-10159-LLWM)

NYHAN, JOHN W. (LS-6)

DRENNON, BARRY J. (LS-6)

ABEELE, WLLY V. (LS-6)

TRUJILLO, GEORGE (LS-6)

HERRERA, WILFRED J. (LS-6)

WHEELER, MERLIN L. (LS-6)

BOOTH, JOHN W. (MST-6)

PURTYMUN, WILLIAM D. (HSE-8)

42-4

EFFECTS OF EXCHANGEABLE CATION

COMPOSITION ON THE THERMAL EXPANSION/ CONTRACTION OF CLINOPTILOLITE.

CLAYS CLAY MINER.,

V.32, P.444-51. 1984.

BISH, DAVID L. (ESS-2)

42-5

FIELD STUDIES AND MODELING OF CHEMICAL PROCESSES IN THE UNSATURATED ZONE

TIC.

1983. 10P. MN (LA-UR-83-2591)

DE POORTER, GERALD L. (LS-6)

LANGHORST, GARY J. (LS-6)

STEGER, JAMES G. (LS-6)

42-6

FIELD STUDIES OF EROSION CONTROL TECHNOLOGIES FOR ARID SHALLOW LAND BURIAL SITES AT LOS ALAMOS.

TIC,

1983. 11P. MN (LA-UR-83-2590)

NYHAN, JOHN W. (LS-6)

ABEELE, WILLY V. (LS-6)

DE POORTER, GERALD L. (LS-6)

HAKONSON, THOMAS E. (LS-6)

PERKINS, BETTY A. (LS-6)

FOSTEA, GEORCE R. (LS-6)
42-7

GEOTECHNICAL ASPECTS OF HACKROY SANDY

LOAM AND CRUSHED TUFF.

LOS ALAMOS.

1984. 20P. (LA-09916-MS)

ABEELE, WILLY V.(LS-6)

$42-8$

GEOTECHNICAL CHARACTERISTICS OF

BENTONITE/SANDY SILT MIXES FOR

USE IN WASTE DISPOSAL SITES.

LOS ALAMOS,

1984. 10P. (LA-10101-MS)

ABEELE, WILLY V. (LS-6)

$42-9$

GROUNDWATER CHEMISTRY ALONG FLOW PATHS

BETWEEN A PROPOSED REPOSITORY SITE AND

THE ACCESSIBLE ENVIRONMENT.

LOS ALAMOS,

1984. 48P. (LA-10t88-MS)

OGARD, ALLEN E. (INC-7)

KERRISK, JERAY F. $(\mathbf{X} \times-4)$

42-10

INFLUENCE OF SOIL BIOPOPULATION ON

MIGRATION OF WASTE RADIONUCLIDES.

TIC.

1983. 9P. MN (LA-UR-63-3385)

FOWLER, ERIC B. (LS-6)

POLZER, WILFRED L (LS-6)

ESSINGTON, EDWARD H. (LS-6)

42-11

LABORATORY AND FIELD STUDIES RELATED TO

THE RADIONUCLIDE MIGRATION PROJECT.

OCTOBER 1, 1982 - SEPTEMBER 30, 1983.

LOSALAMOS.

1984. 33P. (LA-10121-PR)

DANIELS, WILLIAM R. (INC-11)

THOMPSON, JOE L. (INC-11)

42-12

LOS ALAMOS CONTROLLED AIR INCINERATION STUDIES.

TIC,

1983. 15P. MN (LA-UR-83-3024)

KOENIG, RALPH A., JR. (H-7)

WARNER, CHARLES L. (H-7)

42-13

NEW CONTINUOUSLY MONITORED COLLECTION

SYSTEM FOR LUQUID INDUSTRIAL WASTES.

J. WATER POLLUT. CONTROL FED.,

V.56, P.1192-8. 1984.

EMELITY, LUDGARD A. (HSE-7)

SANDERS, WILLIAM MORT (HSE-7)

GARDE, RAYMOND (HSE-7)

BUCHHOLZ, JERRY R. (HSE-7)
42-14

NUMERICAL SIMULATION OF FLOW AND

TRANSPORT IN FRACTURED TUFF.

MATERIALS RESEARCH SOCIETY SYMPOSIA,

1983, BOSTON. PROC., P.1039-46.

ELSEVER, 1984.

TRAVS, BRYAN J. (ESS-5)

HODSON, STEPHEN W. (ESS-5)

NUTTAL HERBERT E

COOK, THOMAS L (ESS-5)

RUNDBERG, ROBERT S. (NC-11)

42-15

NUMERICAL SIMULATION OF FLOW AND

TRANSPORT IN PARTIALLY SATURATED, FRACTURED TUFF.

TIC,

1983. 17P. MN (LA-UR-83-3341)

TRAVS, BRYAN J. (ESS-5)

HODSON, STEPHEN W. (ESS-5)

COOK, THOMAS L. (ESS-5)

NUTTALL, HERBERT, E.

RUNDBERG, ROBERT S. (INC-11)

42-16

PERMEABILTY CHANGES DURING TIMEDEPENDENT DEFORMATION OF SILICATE ROCK.

GEOPHYS. RES. LETT.,

V.11, P.975-8. 1984.

KRANZ, ROBERT

BLACIC, JAMES D. (ESS-3)

42-17

PETROGRAPHY AND MINERAL CHEMISTRY OF UNITS OF THE TOPOPAH SPRING, CALICO HILLS AND CRATER FLAT TUFFS, AND OLDER VOLCANIC UNITS, WITH EMPHASIS ON SAMPLES FROM DRILL HOLE USW G-1. YUCCA MOUNTANN, NEVADA TEST SITE.

LOS ALAMOS,

1984. 78P. (LA-10003-MS)

WARREN, RICHARD G. (ESS-2)

BYERS, FRANKM. (ESS-1)

CAPORUSCIO, FLORIEA. (ESS-1)

42-18

PETROLOGY OF SAMPLES FROM DRIU HOLES USW - H3, H4, H5, YUCCA MOUNTAIN, NEVADA.

LOS ALAMOS,

1984. 76P. (LA-09706-MS)

LEV, SCHON S. (ESS-1)

42-19

PLANT UPTAKE ASSAY TO DETERMINE

BIOAVAILABIUTY OF INORGANIC

CONTAMINANTS.

WATER AR SOIL POLLUT.

V.22, P.85-93. 1984.

DAEESEN, DAVD R. (LS-6)

COKAL, EDWARD J. (LS-6) 
42-20

RESEARCH AND DEVELOPMENT RELATED TO THE NEVADA NUCLEAR WASTE STORAGE

INVESTIGATIONS, JULY 1 - SEPTEMBER

$30,1983$.

LOS ALAMOS,

1984. 100P. (LA-10006-PR)

BRYANT, ERNEST A. (CNC-7)

VANIMAN, DAVDT. (ESS-1)

\section{2-21}

RESEARCH AND DEVELOPMENT RELATED TO THE NEVADA NUCLEAR WASTE STORAGE

INVESTICATIONS, OCTOBER 1 - DECEMBER 31, 1983.

LOS ALAMOS,

1984. 76P. (LA-10032-PR)

WOLFSBERG, KURT (INC-7)

VANIMAN, DAVIDT. (ESS-1)

42-22

ROOTING DEPTHS OF PLANTS ON LOW LEVEL

WASTE DISPOSAL SITES.

LOS ALAMOS,

1984. 23P. (LA-10253-MS)

FOXX, TERALENE S. (LS-6)

TIEANEY, GAILD. (LS-6)

WILLIAMS, JOEL M. (MST-6)

$42-23$

ROOTING DEPTHS OF PLANTS RELATIVE TO BIOLOGICAL AND ENVIRONMENTAL FACTORS.

LOS ALAMOS,

1984. 26P. (LA-10254-MS)

FOXX, TERALENE S. (LS-6)

TIERNEY, GAIL D. (LS-6)

WILLIAMS, JOEL M. (MST-6)
42-24

SHALLOW LAND BURIAL TECHNOLOGY - ARID. TIC,

1983. 15P. MN (LA-UR-83-2589)

ABEELE, WILLYV. (LS-6)

DE POORTER, GERALD L. (LS-6)

HAKONSON, THOMASE. (LS-6)

NYHAN, JOHN W. (LS-6)

42-25

SOLUBILITY LIMITS ON RADIONUCLIDE

DISSOLUTION AT A YUCCA MOUNTAIN

REPOSITORY.

LOS ALAMOS,

1984. 64P. (LA-09995-MS)

KERRISK, JERRY F. (WX-4)

42-26

STUDIES OF ALTERED VITROPHYRE FOR THE PREDICTION OF NUCLEAR WASTE REPOSITORY INDUCED THERMAL ALTERATION AT YUCCA MOUNTAN, NEVADA.

TIC.

1983. 9P. MN (LA-UR-83-3402)

LEY, SCHON S. (ESS-1)

42-27

SURFACE WATER MANAGEMENT - A USER'S GUIDE TO CALCULATE A WATER BALANCE USING THE CREAMS MODEL

LOS ALAMOS,

1984. 49P. (LA-10177-M)

LANE, LEONARD J. (LS-6)
42-28

THERMAL STABILIZATION OF URANIUM MILL

TAUUNGS.

ENVIRON. SCI. TECHNOL,

V.18,P.658-67. 1984

DREESEN, DAVID R. (LS-6).

COKAL, EDWARD J. (LS-6)

WANGEN, LAWRENCEE. (CHM-1)

WILLIAMS, JOEL M. (LS-6)

THODE, EDWARD F.

42-29

VARIATIONS IN AUTHIGENIC MINERALOGY AND

SORPTIVE ZEOLITE ABUNDANCE AT YUCCA

MOUNTAIN, NEVADA, BASED ON STUDIES OF

DRILL CORES USW GU - 3 AND G - 3

LOS ALAMOS,

1984. 71P. (LA-09707-MS)

VANIMAN, DAVID T. (ESS-1)

BISH, DAVD L. (ESS-2)

BROXTON, DAVID E. (ESS-2)

BYERS, FRANK M. (ESS-1)

HEIKEN, GRANT H. (ESS-1)

CARLOS, ROBERT C. (ESS-7)

SEMARGE, R. ELLEN (ESS-2)

CAPORUSCIO, FLORIE A. (ESS-1)

GOOLEY, RONALD C. (ESS-2)

$42-30$

WATER AND CONTAMINANT MOVEMENT

MIGRATION BARRIERS.

LOS ALAMOS,

1984. 19P. (LA-10242-MS)

LANE, LEONARD J. (LS-6)

NYHAN, JOHN W. (LS-6) 


\section{OIL SHALES AND TAR SANDS}

43-1

ASPEN SIMULATION OF OIL SHALE RETORT OFF-GAS CLEANUP WITH VENTURI SCRUBBERS.

LOS ALAMOS,

1984. 14P. (LA-10044-MS)

PHILLIPS, THOMAS TERRENCE $(W X-4)$

43-2

ECONOMIC COMPARISON OF FIVE PROCESS

CONCEPTS FOR USING EASTERN OIL SHALE.

TIC,

1984. 21P. MN (LA-UR-83-3041)

PARKINSON, WILLIAM J. (WX-4)

PHILLIPS, THOMAS TERRENCE $(W X-4)$

BARNES, JAMES $W .(W X-4)$
43-3

ECONOMIC COMPARISON OF FIVE PROCESS

CONCEPTS FOR USING EASTERN OIL SHALE. LOS ALAMOS,

1984. 102P. (LA-10106-MS)

PARKINSON, WILLIAM J. (WX-4)

PHILLIPS, THOMAS TERRENCE $(W X-4)$ BARNES, JAMES W. (Q-4)

\section{3-4}

ROLE OF COMPUTER SIMULATION IN OIL SHALE BLASTING.

TIC,

1984. BP. MN (LA-UR-64-1069)

ADAMS, THOMAS F. (ESS-5)

KELLER, CHARLES F. (ESS-5)
43-5

STATISTICAL CRACK MECHANICS.

TIC,

1983. 21P. MN (LA-UR-83-1705)

DIENES, JOHN K. (T-3)

43-6

SUMMARY OF THE OIL SHALE FRAGMENTATION PROGRAM AT ANVIL POINTS MINE, COLORADO.

TIC,

1984. 26P. MN (LA-UR-84-1558)

DICK, RICHARD DEAN (ESS-3)

YOUNG, CHAPMAN

FOURNEY, WILLIAM L. 


\section{PARTICLE ACCELERATORS AND HIGH VOLTAGE MACHINES}

44-1

ACCELERATOR TECHNOLOGY PROGRAM,

JULY - DECEMBER 1982.

LOS ALAMOS,

1984. 88P. (LA-10118-SR)

JAMESON, ROBERT A. (AT-DO)

44-2

ACCELERATOR TECHNOLOGY PROGRAM, JANUARY - SEPTEMBER 1983.

LOS ALAMOS

1984. 85P. (LA-10191-SR)

JAMESON, ROBERT A. (AT-DO)

44-3

APPLYING ACCELERATOR REMOTE TECHNOLOGY TO FUSION DEVICES.

TRANS. AM. NUCL. SOC.,

V.44, P.571-2. 1983.

ALSO PUBLISHED IN

TIC,

1983. 11P. MN (LA-UR-83-1558)

ALSO PUBLISHED IN:

CONFERENCE ON REMOTE SYSTEMS

TECHNOLOGY, 31ST, 1983, DETROIT.

PROC., P.55-8. ANS, 1984.

GRISHAM, DONALD L. (MP-7)

LAMBERT, JAMES E. (MP-7)

44-4

ARCHSIM - A PROTON SYNCHROTRON TRACKING PROGRAM INCLUDING LONGITUDINAL SPACE CHARGE.

TIC.

1983. 7P. MN (LA-UR-83-2703)

THIESSEN, HENRY A. (MP-DO)

WARREN, JOHN L. (AT-6)

44-5

BEAM CAVITY INTERACTION.

LNEAR ACCELERATOR CONFERENCE,

1984, SEEHEIM: PROC., P.265-7.

1984. (GSI-84-11)

GLUCKSTERN, ROBERT L.

COOPER, RICHARD K. (AT-6)

44-6

BEAM PIPE DESIGN, WALL - HEATING, AND COLLECTIVE INSTABILITY CONSTRAINTS FOR

LAMPF $\|$.

LOS ALAMOS

1984. 5P. (LA-10223-MS)

NEUFFER, DAVID (AT-6)

44-7

DESIGN FEATURES AND PERFORMANCE OF THE

LAMPF HIGH INTENSITY BEAM AREA.

TIC,

1983. 7P. MN (LA-UR-83-2693)

AGNEW, LEWIS E., JR. (MP-DO)

GAISHAM, DONALD L. (MP-7)

MACEK, ROBERT J. (MP-13)

SOMMER, WALTER F., JR. (MP-13)

WERBECK, RICHARD D. (MP-7)
44-8

DESIGN OF FERRITE - TUNED ACCELERATOR CAVITIES USING PERPENDICULAR - BIASED HIGH - Q FERRITES.

LOS ALAMOS,

1984. 18P. (LA-10277-MS)

KASPAR, KLAUS

44-9

DESIGNING SELF - MATCHING LINACS.

UNEAR ACCELERATOR CONFERENCE,

1984, SEEHEIM, PROC., P.112-4.

1984. (GSI-84-11)

MILLS, RENE SCOTT (AT-2)

CRANDALL; KENNETHR. (AT-1)

FARRELL, JOHN ALOEN (AT-2)

44-10

DEVELOPING A 500 EV PROTON BEAM.

POLARIZED PROTON ION SOURCES, 1983,

VANCOUVER. PROC., P.82-93. AMER.

INST. OF PHYSICS, 1984.

(539.72/P762PR/1983)

ALSO PUBLISHED IN

TIC,

1983. 13P. MN (LA-UR-83-1466)

CHAMBERLIN, EDWIN P. (MP-11)

BENAGE, JOHN F., JR. (MP-11)

WILLIAMS, HARRY EUGENE (MP-11)

44-11

DEVELOPMENT OF A MULTICUSP HYDROGEN MINUS ION SOURCE FOR ACCELERATOR APPLICATIONS.

INTL. SYMPOSIUM ON PRODUCTION AND NEUTRALIZATION OF NEGATIVE IONS AND BEAMS, 3RD, 1983, EROOKHAVEN. PROC., P.410-7. AMER. INST. OF PHYSICS

1984. (539.73/P964NE/1983)

ALSO PUBLISHED IN:

TIC.

1983. 8P. MN (LA-UR-83-3273)

YORK, ROBBIE LYNN (MP-11)

STEVENS, RALPH R., JR. (MP-DO)

44-12

DIPOLE APERTURE AND SUPERCONDUCTOR REQUIREMENTS

TIC,

1983. 5P. MN (LA-UR-84-0096)

WIPF, STEFAN L. (P-DO)

44-13

EFFET OF RF STRUCTURE ON CUMULATIVE

BEAM BREAKUP.

TIC.

1983. 5P. MN (LA-UR-83-2317)

GLUCKSTERN, ROBERT L:

COOPER, RICHARD K. (AT-6)

CHANNELL, PAUL J. (AT-6)
44-14

ELECTRON BEAM DIAGNOSTICS FOR THE LOS ALAMOS FREE-ELECTRON OSCILLATOR EXPERIMENT.

FREE-ELECTRON GENERATORS OF

COHERENT RADIATION, 1983.

ORCAS ISLAND, WASHINGTON, PROC.

P. 151-2. SPIE, 1984.

(621.366/F853EL/1983)

ALSO PUBLSHED IN

TIC.

1983. 6P. MN (LA-UR-83-2383)

SHEFFIELD, RICHARD L. (AT-7)

STEIN, WILLIAM E. (AT-7)

WARREN, ROGER W. (AT-7)

LUMPKIN, ALEX H. (P-DO)

FRASER, JOHN S. (AT-7)

44-15

ELECTRON MOTION IN SOLENOIDAL MAGNETIC FIELDS USING A FIRST-ORDER SYMPLECTIC INTEGRATION ALGORITHM.

LINEAR ACCELERATOR CONFERENCE,

1984, SEEHEIM. PROC., P.206-8.

1984. (GSI-84-11)

FRASER, JOHN S. (AT-7)

44-16

EXPLORATION OF PHASE STABILITY IN THE SURFATRON ACCELERATOR.

LINEAR ACCELERATOR CONFERENCE,

1984, SEEHEIM. PROC., P.162-4.

1984. (GSI-84-11)

ALSO PUBLISHED IN:

TIC

1984. 4P. MN (LA-UR-84-1349)

NEUFFER, DAVID (AT-6)

44-17

GENERAL-PURPOSE RFO DESIGN PROGRAM.

LINEAR ACCELERATOR CONFERENCE,

1984. SEEHEIM, PROC., P. 330-1.

1984. (GSI-84-11)

WADLINGER, E. ALAN (AT-2)

44-18

HIGH - FIELD DIPOLES FOR FUTURE

ACCELERATORS

LOS ALAMOS,

1984. 45P. (LA-10219-MS)

WIPF, STEFAN L. (AT-6)

44-19

HIGH - Q PERPENDICULAR BIASED

FERRITE - TUNED CAVITY.

TIC,

1983. 4P. MN (LA-UR-83-2445)

CARLINI, ROGER D. (MP-4)

THIESSEN, MENRY A. (MP-DO)

POTTER, J. M. (AT-1) 
44-20

HIGH POWER TESTING OF THE MULTIPLE LOOP RADIO FREQUENCY DRIVE CONCEPT FOR THE

FMIT ACCELERATOR.

UNEAR ACCELERATOR CONFERENCE,

1984, SEEHEIM. PROC., P.183-5

1984. (GSI-84-11)

ALSO PUBLISHED IN:

T1C.

1984. 4P. MN (LA-UR-84-1348)

FAZIO, MICHAEL V. (AT-5)

PATTON, ROBERT D. (AT-5)

44-21

HIGH-Q PERPENDICULAR-BIASED FERRITE

TUNED CAVITY.

LOS ALAMOS,

1984. 4P. (LA-09946-MS)

CARLINI, ROGER D. (MP-4)

THIESSEN, HENRY A. (MP-DO)

POTTER, J. M. (AT-1)

44-22

HUMAN FACTORS CONSIDERATIONS IN THE

MONITOR SYSTEMS.

TRANS. AM. NUCL. SOC.,

V.44, P.568-9. 1983.

ALSO PUBLISHED IN:

TIC,

1983. 12P. MN (LA-UR-83-1559)

ALSO PUBLISHED IN:

CONFERENCE ON REMOTE SYSTEMS

TECHNOLOGY, 30TH, 1982, LOS ANGELES.

PROC., P.35-9. ANS, 1984.

GRISHAM, DONALD L. (MP-7)

LAMBERT, JAMES E. (MP-7)

44-23

INSTRUMENTATION AND CONTROL SYSTEM FOR

THE AT - 2 ACCELERATOR TEST STAND.

LINEAR ACCELERATOR CONFERENCE,

1984, SEEHEIM, PROC., P.150-2.

1984. (GSI-84-11)

ALSO PUBLISHED IN:

TIC.

1984. 4P. MN (LA-UR-84-1278)

WADLINGER, E. ALAN (AT-2)

HOLTKAMP, DAVID B. (AT-2)

HOLT, HAL D. (AT-2)

44-24

INTRODUCTION OF RFQ SESSION.

LINEAR ACCELERATOR CONFERENCE,

1984, SEEHEIM. PROC. P.49-52.

1984. (GSI-84-11)

ALSO PUBLISHED IN:

TIC,

1984. SP. MN (LA-UR-84-1375)

JAMESON, ROBERT A. (AT-DO)

44-25

INVESTIGATON OF EMITTANCE GROWTH IN THE WHITE HORSE BEAM.

LOS ALAMOS

1984. 14P. (LA-09953-MS)

JONES, MICHAEL E. (X-8)

LEE, HUAN $(X-8)$

LEMONS, DON S. $(X-8)$
44-26

LAMPF TRANSITION - RECION MECHANICAL

FABRICATION.

LOS ALAMOS

1984. 51P. (LA-10136-MS)

BUSH, EDGAR D., JR. (MP-8)

GALLEGOS, JOSE D. F. (MP-8)

HARRISON, RONALD (MP-8)

HART, VALGENE E. (MP-8)

HUNTER, W. TED (MP-8)

RISLOVE, SETHE. (MP-8)

SIMS, JAMES R. (MP-8)

VAN DYKE, W. JOSEPH (MP-8)

44-27

LATTICE FOR A LOW - FIELD

SUPERCONDUCTING SUPER COLLIDER.

TIC,

1983. 4P. MN (LA-UR-84-0191)

NEUFFER, DAVID (AT-6)

44-28

LINAC - DRIVEN XUV FREE - ELECTRON

LASER.

FREE ELECTRON GENERATION OF EXTREME

ULTRAVOLET COHERENT RADIATION,

1983, BROOKHAVEN. PROC., P.190-202. AP, 1984. (621.366/F853EL/1983)

ALSO PUBLISHED IN:

TIC,

1983. 24P. MN (LA-UR-83-3288)

NEWNAM, BRIANE. (CHM-6)

GOLDSTEIN, JOHN C. (X-1)

FRASER, JOHN S. (AT-7)

COOPER, RICHARD K. (AT-6)

44-29

LINER - DRIVEN RING ACCELERATORS

LOS ALAMOS

1984. 8P. (LA-09968-MS)

GISLER, GALEN $(X-8)$

FAEHL, R. J. (X-8)

44-30

LONGITUDINAL SPACE - CHARGE EFFECTS IN

BUNCHED BEAMS

TIC,

1983. 8P. MN (LA-UR-83-2339)

CHANNELL, PAUL J. (AT-6)

44-31

LUMPED - CIRCUIT MODEL OF FOUR - VANE

RFQ RESONATOR.

UNEAR ACCELERATOR CONFERENCE,

1984, SEEHEIM. PROC., P.332-4.

1984. (GSI-84-11)

WANGLER, THOMAS P. (AT-1)

$44-32$

MAGNETS FOR THE PROTON STORAGE RING

ATLOS ALAMOS.

J. PHYS. COLLOO.

No.1, P.1-245. 1984.

ALSO PUBUSHED IN:

TIC.

1983. 5P. MN (LA-UR-83-2477)

HARVEY, ALEXANDER (AT-5)
$44-33$

NEGATIVE HYDROGEN ION CURRENT FROM A POLARIZED VAPOA TARGET.

TIC.

1984. 9P. MN (LA-UR-84-0990)

CORNELIUS, WAYNE D. (AT-4)

$44-34$

NEW DIRECTIONS IN LINEAR ACCELERATORS.

UNEAR ACCELERATOR CONFERENCE,

1984, SEEHEIM. PROC., P.237-42.

1984. (GSI-84-11)

JAMESON, ROBERT A. (AT-DO)

44-35

NEW UNAC TECHNOLOGY - FOR SSC, AND BEYOND.

TIC,

1983. 6P. MN (LA-UR-83-2365)

JAMESON, ROBERT A. (AT-DO)

44-36

OPERATING EXPERIENCE WITH A 100 - KEV, 100 MA HYDROGEN MINUS INJECTOR.

WNTL. SYMPOSIUM ON PRODUCTION AND NEUTRALIZATION OF NEGATTVE IONS AND BEAMS, 3RD, 1983, BROOKHAVEN. PROC., P.5 11-8. AMER. INST. OF PHYSICS.

1984. (539.73/P964NE/1983)

ALSO PUBLISHED IN

TIC.

1983. 9P. MN (LA-UR-83-3254)

ALLISON, PAUL W. (AT-2)

SHERMAN, J. D.

$44-37$

OPERATIONAL PARAMETERS OF A 2.0 MEV RFO

UNAC.

UNEAR ACCELERATOR CONFERENCE,

1984, SEEHEIM, PROC., P.54-5.

1984. (GSI-84-11)

SANDER, OSCAR R. (AT-2)

PURSER, FRED O. (AT-2)

RUSTHOI, DANIEL P.

44-38

OPTICAL ALIGNMENT TO SET A SKEWED

BEAMLNE FOR NEUTRINO RESEARCH AT THE

LAMPF ACCELERATOR.

OPTICAL ALIGNMENT II, 1984

ARLINGTON. PROC., P.131-8. SPIE,

1984. (621.36/062A/1984)

BAUKE, WALTER (P-5)

CLARK, DAVID A. (P-9)

TRUJILLO, PETER B. (P-9)

44-39

OPTICALLY PUMPED POLARIZED HYDAOGEN MINUS ION SOURCE.

INTL. SYMPOSIUM ON PRODUCTION AND NEUTRAUZATION OF NEGATIVE IONS AND BEAMS, 3RD, 1983, BROOKHAVEN. PROC. P.769-71. AMER. INST. OF PHYSICS,

1984. (539.73/P964NE/1983)

MORI, YOSHIHARU

IKEGAMI, KYYOSHI

TAKAGI, AKIRA

IGARASHI, $\mathbf{Z}$.

FUKUMOTO, SADAYOSHI

CORNELUUS, WAYNE D. (AT-4)

YORK, ROBBIE LYNN (MP-11) 
44-40

PARTICLE ACCELERATOR CONTROL AND DATA ACQUISITION IN THE CONTEXT OF VAXMMS.

TIC,

1983. 6P. MN (LA-UR-83-3444)

SCHALLER, STUART C. (MP-1)

CORLEY, JAMII K. (MP-1)

44-41

PATH - A LUMPED - ELEMENT BEAM

TRANSPORT SIMULATION PROGRAM WITH SPACE CHARGE.

TiC,

1983. 7P. MN (LA-UR-83-2702)

FARAELL, JOHN ALDEN (AT-2)

44-42

PHASE STABILITY IN SURFATRON

ACCELERATION.

TIC.

1983. 5P. MN (LA-UR-83-2366)

NEUFFER, DAVID (AT-6)

44-43

PRACTICAL ASPECTS OF TUNING A RINGED RFQ.

UNEAR ACCELERATOR CONFERENCE,

1984, SEEHEIM, PROC., P.115-7.

1984. (GSI-B4-11)

PURSER, FRED O. (AT-2)

POTTER, J. M. (AT-1)

HUMPHREY, FREDERICK J. (AT-5)

44-44

PRACTICAL ASPECTS OF TUNING A RINGED

RFO.

TIC,

1984. 4P. MN (LA-UR-64-1308)

PURSER, FRED O. (AT-2)

POTTER, J. M. (AT-1)

HUMPHRY, FREDERICK J. (AT-1)

$44-45$

PRINCIPLES AND APPLICATIONS OF MUON COOLNG.

TIC.

1983. 6P. MN (LA-UR-83-2375)

NEUFFER, DAVID (AT-6)

44-46

PROGRESS AT LAMPF, JANUARY - DECEMBER

1983, CLINTON P. ANDERSON MESON PHYSICS

FACILITY.

LOS ALAMOS,

1984. 269P. (LA-10070-PR)

ALLRED, JOHN C. (MP-DO)

44-47

PROTON STORAGE RING (PSR) DIAGNOSTICS AND CONTROL SYSTEMS.

TIC,

1983. 7P. MN (LA-UR-83-2592)

CLOUT, PETER N. (AT-3)

44-48

PSR BEAM PULSE FORMATION AND CONTROL.

TIC.

1983. TP. MN (LA-UR-83-2593)

HARDEKOPF, ROBERT A. (AT-3)
44-49

RADIAL PARTICLE DISTRIBUTIONS IN

PARMILA SIMULATION BEAMS.

LOS ALAMOS.

1984. 34P. (LA-10063-MS) BOICOURT, GRENFELLP. (AT-6)

44-60

RADIATION-RESISTANT EEAMLNE

COMPONENTS AT LAMPF.

TIC,

1983. 7P. MN (LA-UR-83-2736)

MACEK, ROBERT J. (MP-13)

GRISHAM, DONALD LL (MP-7)

LAMBERT, JAMES E. (MP-7)

WERBECK, RICHARD D. (MP-7)

44-51

REFERENCE DESIGN FOR LAMPF $\|$.

TIC,

1983. 5P. MN (LA-UR-83-2426)

THIESSEN, HENRY A. (MP-DO)

$44-52$

REMOTE HANDLING AT LAMPF.

TIC.

1983. SP. MN (LA-UR-83-2692)

GRISHAM, DONALO L. (MP-7)

LAMBERT, JAMES E. (MP-7)

$44-53$

REMOTE OPERATIONS AND VIEWING USING

THE MONITOR SYSTEM.

TRANS. AM. NUCL. SOC.

V.47, P.456. 1984

GRISHAM, DONALD L (MP-7)

LAMBERT, JAMES E. (MP-7)

BALDWN, THOMAS S. (MP-7)

EKBERG, ETHEN L (MP-7)

HERNANDEZ, TOMMY R. (MP-7)

RAYBUN, JOSEPHL (MP-7)

44-54

REMOTE REPLACEMENT OFA TARGET CELL

AT LAMPF.

TRANS. AM. NUCL. SOC.,

V.46, P.773-4. 1984.

ALSO PUBLISHED IN:

TIC,

1984. 6P. MN (LA-UR-84-1626)

GRISHAM, DONALDL. (MP-7)

LAMBERT, JAMES E. (MP-7)

44-55

RFQ DEVELOPMENT AT LOS ALAMOS

TIC.

1984. 6P. MN (LA-UR-84-0498)

ARMSTRONG, DALE D. (AT-4)

CORNELUS, WAYNE D. (AT-4)

PURSER, FRED O. (AT-2)

JAMESON, ROBERT A. (AT-DO)

WANGLER, THOMAS P. (AT-1)

44-56

RFQ RADIAL MATCHING SECTIONS AND FRINGE

FIELDS.

LNEAR ACCEL ERATOR CONFERENCE,

1984, SEEHEIM. PROC., P.109-11.

1984. (CS1-84-11)

ALSO PUBLISHED IN

TIC.

1984. 4P. MN (LA-UR-84-1281)

CRANDALL, KENNETH R. (AT-1)
44-57

RING ACCELERATORS

TIC.

1983. 5P. MN (LA-UR-83-2588)

GISLER, CALEN $(X-8)$

FAEHL, R. J. (X-8)

$44-58$

SCALED, CIRCULAR EMITTER PENNING SPS

FOR INTENSE HYDROGEN MINUS BEAMS.

INTL. SYMPOSIUM ON PRODUCTION AND

NEUTRALIZATION OF NEGATIVE IONS AND

BEAMS, 3RD, 1983, BROOKHAVEN. PROC.,

P.458-62. AMER. INST. OF PHYSICS,

1984. (539.73/P964NE/1983)

ALSO PUBLISHED IN

no.

1983. 6P. MN (LA-UR-83-3246)

SMTTH, HORACE V. (AT-2)

ALUSON, PAUL W. (AT-2)

SHERMAN, JOSEPH D. (AT-2)

4469

SIMULATION OF TRANSITION CROSSING IN

LAMPF II.

TIC,

1983. 4P. MN (LA-UR-83-2367)

WARREN, JOHN L. (AT-6)

THIESSEN, HENAY A (MP-14)

44-60

SPALLATION RADIATION DAMAGE AND THE RADIATION DAMAGE FACILITY AT THE LAMPF

A-6 TARGET STATION.

J. NUCL, MATER.

V.122-3,P.1078-84. 1984

WECHSLER, M. $\mathbf{S}$.

SOMMER, WALTER F., JR. (MP-13)

44-61

SSC RF DESIGN EXAMPLE.

TC.

1983. 4P. MN (LA-UR-84-0327)

COLTON, EUGENE P. (MP-14)

44-62

STAGED APPROACH FOR LAMPF II.

LOS ALAMOS.

1984. 23P. (LA-09949-MS)

COLTON, EUGENE P. (MP-14)

44-63

STATUS OF THE NEW HIGH INTENSITY

HYOROGEN NEGATIVE INJECTOR AT LAMPF. UNEAR ACCELERATOR CONFERENCE, 1984, SEEHEIM, PROC., P.226-8.

1884. (GSI-84-11)

ALSO PUBLISHED IN:

Nic.

1984. 4P. MN (LA-UR-84-1232)

STEVENS, RALPH R., JA. (MP-DO)

YORK, ROBBIE LYNN (MP-11)

MC CONNELL, JOHN R. (MP-12)

KANDARIAN, ROBERT (MP-11)

44-64

STATUS REPORT ON FMTT

UINEAR ACCELERATOR CONFERENCE,

1984, SEEHEIM. PROC., P.505-6.

1984. (GSI-84-11)

ARMSTRONG, DALED. (AT-4) 
44-65

SUBHARMONIC BUNCHER FOR THE LOS ALAMOS

FREE - ELECTRON LASER OSCILLATOR

EXPERIMENT.

FREE-ELECTRON GENERATORS OF

COHERENT RADIATION, 1983,

ORCAS ISLAND, WASHINGTON. PROC.

P. 146-50. SPIE, 1984.

(621.366/F853EL/1983)

ALSO PUBLISHED IN:

TIC,

1983. 1OP. MN (LA-UR-83-2151)

FRASER, JOHN S. (AT-7)

44-66

THIRTY - FIVE YEARS OF DRIFT - TUBE

LINAC EXPERIENCE.

LOS ALAMOS,

1984. 14P. (LA-10138-MS)

KNOWLES, HARROLD B. (MP.3)
44-67

TRANSITIONLESS LATTICES FOR LAMPF II.

LOS ALAMOS,

1984. 58P. (LA-10260-MS)

FRANCZAK, BERNHARD J.

44-68

VACUUM BREAKDOWN AND SURFACE COATING OF RF CAVITIES.

J. APPL. PHYS.

V.56,P.1546-7. 1984.

PETER, WILLIAM K. $(X-8)$
44-69

VACUUM REQUIREMENTS FOR LAMPF

LOS ALAMOS,

1984. 8P. (LA-10224-MS)

NEUFFER, DAVID (AT-6) 


\section{PETROLEUM AND NATURAL GAS}

45-1

LABORATORY ULTRASONIC AND RESISTIVITY MEASUREMENTS ON SEDIMENTARY ROCKS CONTAINING TETRAHYDROFURAN HYDRATES. LOS ALAMOS,

1984. 67P. (LA-09972-MS)

PEARSON, CHRISTOPHER F. (ESS-3)

MURPHY, J. (ESS-3)

HERMES, ROBERT E. (ESS-2)

HALLECK, PHILLIP M. (ESS-3)
45-2 NUCLEAR MAGNETIC RESONANCE WELLLOGGING.

LOG ANAL.

V.25, NO.5, P.16-30. 1984. JACKSON, JASPER A., JR. (ESS-3)
45-3

NUMERICAL METHODS DEVELOPED FOR THE SIMULATION OF COUPLED HIGH - ENERGY GAS FLOWS AND STRESS WAVE PROPAGATION IN GEOLOGIC MEDIA.

SHOCK WAVES IN CONDENSED MATTER, 1983, SANTA FE. PROC., P.505-8.

ELSEVIER, 1984.

KILLIAN, BARBARA GERMAIN (G-DO) 


\section{PHYSICS-ATOMIC AND MOLECULAR}

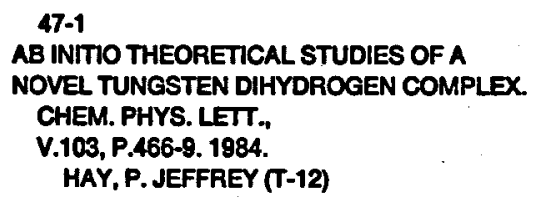

47-7

CHARACTERIZATION OF THE FIRST EXAMPLES OF ISOLABLE MOLECULAR HYDROGEN COMPLEXES, METAL (TRICARBONYL) (ORGANOPHOSPHINE)2 (DIHYDROGEN) (METAL EQUALS MOLYBDENUM, TUNGSTEN: ALKYL EQUALS CYCLOHEXYL, ISOPROPYL). EVIDENCE FOR A SIDE-ON BONDED DIHYDROGEN LIGAND.

J. AM. CHEM. SOC.,

V.106, P.451-2. 1984. KUBAS, GREGORY J. (INC-4)

RYAN, ROBERT R. (INC-4)

SWANSON, BASIL I. (INC-4)

VERGAMINI, PHILLIP J. (P-8)

WASSERMAN, HARVEY (INC-4)

47-8

CLASSICAL STOCHASTIC DIFFUSION THEORY FOR THERMAL DESORPTION FROM SOLDD SURFACES.

J. VAC. SCI. TECHNOL.

V.B2, P.550-60. 1984

REDONDO, ANTONIO (E-11)

ZEIRI, YEHUDA

GODDARD, WULIAM A., III

47-9

COMMENT ON "GENERIC BEHAVIOR OF

COUPLED OSCIULATORS."

PHYS. REV.

V.30A, P.1127. 1984.

MAYER-KRESS, GOTTFRIED (CNLS)

HAUBS, $G$.

47-10

COMPARATIVE STUDY OF LOW-ENERGY 2 SIGMA G AND 2 PI G SCATTERING IN MOLECULAR NITROGEN.

PHYS. REV.

V.30A, P.95-9. 1984.

SCHNEIDER, BARRY I. (T-12)

COLUNS, LEE A. (T-4)

47-11

CORRELATION EFFECTS ON CHARGE-DENSITY WAVES IN NARROW-BAND ONE-DIMENSIONAL

CONDUCTORS.

PHYS. REV.

V.30B, P.4842-5. 1984

MAZUMDAR, SUMIT (CNLS)

DIXIT, S. N.

BLOCH, A. N.

47-12

DEEP CORE - LEVEL RESONANCE

PHOTOEMISSION FROM URANIUM DIOXIDE(111). STANFORD SYNCHROTRON RADIATION LAB. ACTIVITY REPORT FOR APRIL 1, 1982 MARCH 31, 1983. K. CANTWELL, ED. P.VII-85. SSRL, 1983. (SSRL-83/01) ALLEN, JAMES W. (P-APO) OH, SE-JUNG EUS, WALTON P. (CHM-2) COX, LAWRENCE E. (MST-13)
47-13

DOPPLER-FREE TWO-PHOTON SPECTROSCOPY OF THE 2 V 3 BAND OF SULFUR HEXAFLUORIDE.

J. MOL. SPECTROSC.,

V.108, P.31-41. 1984

PATTERSON, CHRIS W. (T-12)

HERLEMONT, F.

AZIZI, A.

LEMAIRE, J.

47-14

ELASTIC SCATTERING OF ELECTRONS BY MOLECULES.

ELECTRON-MOLECULE INTERACTIONS AND THEIR APPLICATIONS, LG.

CHRISTOPHOROU, ED., P.1-153. ACADEMIC

PRESS, 1984. (539.721/ES8MON.1)

CSANAK, G. Y. (T-4)

CARTWRIGHT, DAVID C. (CHM-DO)

SRIVASTAVA, S. $K$.

TRAJMAR, $\mathbf{S}$.

47-15

ELASTIC SCATTERING OF POLARIZED PROTONS ON DEUTERIUM AT $800 \mathrm{MEV}$.

LOS ALAMOS,

1984. 175P. (LA-10174-T) WESTON, GARY S.

47-16

ELECTRON - ELECTRON INTERACTIONS AND BROKEN SYMMETRY IN ONE DIMENSION.

TIC.

1983. 16P. MN (LA-UR-83-3014) MAZUMDAR, SUMIT (CNLS) DIXIT, S. N.

47-17

ELECTRON - IMPACT EXCITATION OF SOME LOW - LYING LEVELS OF NEON.

PHYS. REV.,

V.29A, P.1811-23. 1984

MACHADO, LUIZE.

LEAL, EMERSONP.

CSANAK, G. Y. (T-4)

47-18

ELECTRON CORRELATION EFFECTS ON THE NITROGEN-NITROGEN INTERACTION.

J. CHEM. PHYS.

V.81, P.1360-71. 1984

HAY, P. JEFFREY (T-12)

PACK, RUSSEL T. (T-12)

MARTIN, RICHARD L (T-12)

47-19

ELECTRON-GAS PLUS DAMPED-DISPERSION MODEL FOR INTERMOLECULAR FORCES

THE RARE-GAS AND HYDROGEN-HEUUM, HYDROGEN-NEON, AND HYDROGEN-ARGON POTENTIALS.

J. PHYS. CHEM.,

V.88, P.4272-8. 1984 LESAR, RICHARD A (T-11) 
47-20

ELECTRON-IMPACT-EXCITATION CROSS

SECTIONS FOR ELECTRONIC LEVELS IN NEON

FOR INCIDENT ENERGIES BETWEEN 25 AND $100 \mathrm{EV}$.

PHYS. REV.,

V.29A, P.1783-1809. 1984

REGISTER, D.F.

TRANMAR, $S$.

STEFFENSEN, $G$.

CARTWRIGHT, DAVID C. (CHM-DO)

47.21

ELECTRONIC PROPERTIES OF ARGON AND XENON UNDER PRESSURE.

TIC.

1983. 5P. MN (LA-UR-83-2305)

LESAR, RICHARD (P-10)

47-22

EVIDENCE FOR A PHENOMENOLOGICAL SUPERSYMMETRY IN ATOMIC PHYSICS.

PHYS. REV. LETT.,

V.53,P.2285-8. 1984

KOSTELECKY, VACLAV ALAN (T-B)

NIETO, MICHAEL MARTIN (T-B)

47-23

FORCE - FIELD MODEL FOR THE

STRETCHING ANHARMONICITIES OF

SULFUR HEXAFLUORIDE.

J. PHYS. CHEM.

V.88, P.564-74. 1984.

KROHN, BURTON J. (T-12)

OVEREND, JOHN

47.24

HYPERFINE STRUCTURE OF QUARKONIUM

STATES.

PHYS. REV

V.29D, P.513-6. 1984

AERTS, ADRIAN T. (T-5)

HELLER, LEON (T-5)

47-25

IMPROVED ELECTRON - GAS MODEL

CALCULATIONS OF SOLID NITROGEN 2 TO

10 GPA.

J. CHEM. PHYS.,

V.81, P.5104-8. 1984.

LESAR, RICHARD A (T-11)

47-26

INELASTIC COLLISIONS WITHOUT USE OF

TARGET STATES.

CHEM. PHYS. LETT.

V.104,P.129-34. 1984

DROLSHAGEN, GERHARD (T-12)

HELLER, ERIC J. (T-12)

47-27

INTERACTING CHARGE - OROERED STATES

IN A CORRELATED DIATOMIC POLYMER.

PHYS. REV.

V.29B, P.2317-20. 1984

MAZUMDAR, SUMIT (CNLS)

DIXIT, S. N.
47-28

INTERACTION HAMILTONIAN OF QUANTUM

OPTICS.

J. OPT. SOC. AM.

V.1B, P.116-9. 1984

ACKERHALT, JAY R. (T-12)

MILONNI, PETER W. (T-12)

47-29

INTERACTION MATRIXELEMENTS FOR RESONANT

MUONIC - MOLECULE FORMATION.

PHYS. REV. LETT.

V.53, P.738. 1984

COHEN, JAMES S. (T-12)

MARTIN, RICHARD L. (T-12)

47-30

INTRACAVTY ATOMIC ABSORPTION WITH AN

INDUCTIVELY COUPLED PLASMA ATOMIZER.

APPL. SPECTROSC.

V.38, P.876-9. 1984.

DOWNEY, STEPHEN W. (CHM-2)

NOGAR, NICHOLAS S. (CHM-2)

47-31

ISOTOPE SHIFTS AND FORCE FIELD FOR CARBON TETRACHLORIDE IN A KRYPTON MATRIX.

J. PHYS. CHEM.,

V.88, P.5560-3. 1984.

JONES, LLEWELLYN H. (INC-4)

SWANSON, BASILI. (INC-4)

EKBERG, SCOTT A. (CNC-4)

47-32

UNE WIOTH AND LINE SHAPE ANALYSIS IN

THE INDUCTIVELY COUPLED PLASMA BY HIGH

RESOLUTION FOURIER TRANSFORM

SPECTROMETRY.

TIC.

1984. 37P. MN (LA-UR-83-3713)

FAIRES, LYNDA M. (CHM-1)

PALMER, BYRON A. (CHM-1)

BRAULT, J.W.

47-35

MOLECULAR PHOTOIONIZATION IN THE LINEAR

ALGEBRAIC APPROACH - HYDROGEN, NITROGEN,

NITROGEN OXYGEN, AND CARBON DIOXIDE.

PHYS. REV.

V.29A, P.1695-1707. 1984

COLLINS, LEE A. (T-4)

SCHNEIDER, BARRY I. (T-12)

47-34

MOLECULAR PROPERTIES FROM MCSCF-SCEP

WAVE FUNCTIONS. II. CALCULATION OF

ELECRONIC TRANSITION MOMENTS.

J. CHEM. PHYS.,

V.80, P.5080-4. 1984.

WERNER, HANS-JOACHIM (T-12)
$47-35$

MULTIPHOTON ELECTRONIC SPECTROSCOPY OF ATOMS AND SMAU MOLECULES.

ADV. LASER SPECTROSC.

V.2, P.105-34. 1983

GLOWNIA, JAMES H. (CHM-4)

HARTFORD, ALLEN, JR. (CHM-DO)

LOGE, GARY WAYNE (CHM-4)

SANDER, ROBERT K. (CHM-4)

TIEE, JOE J. (CHM-4)

WAMPLER, FRED B. (CHM-4)

47-36

MULTIREFERENCE-CHLORIDE CALCULATIONS OF RADIATIVE TRANSITION PROBABILITIES

CARBON 2.

J. CHEM. PHYS.,

V.80, P.5085-8. 1984.

ROSMUS, PAVEL

WERNER, HANS-JOACHIM (T-12)

47-37

MUON SPIN RELAXATION MEASUREMENTS OF SPIN - CORRELATION DECAY IN SPIN - GLASS

SILVER MANGANESE.

HYPERFINE INTERACT.

V.17-19, P.463-6. 1984.

ALSO PUBLISHED IN:

TIC,

1983. 9P. MN (LA-UR-83-1028)

HEFFNER, ROBERT H. (MP-3)

COOKE, D. W.

LEON, MELVIN (MP-3)

SCHILLACI, MARIOE. (MP-3)

MACLAUGHLIN, DOUGLAS E.

GUPTA, LAXMI C.

$47-38$

OBSERVATION AND ANALYSIS OF FUNDAMENTAL BENDING MODE OF TRITIUM 2 OXYGEN.

J. MOL. SPECTROSC.

V.103, P.41-55. 1984.

FRY, HERBERT A. (INC-4)

JONES, LLWELLYN H. (INC-4)

BAREFIELD, J. E., II (CHM-4)

47-39

OPTIC PHONONS IN NICKEL HYDRIDE

PHYS. REV.

V.29B, P.3700-2. 1984

ECKERT, JUERGEN (P-8)

MAJKZRAK, CHARLES F.

PASSELL, LAURENCE

DANIELS, WILLIAM

47-40

ORIENTATIONAL ORDERING AND SITE

STRUCTURE FOR CARBON CHLORIDE INA

KRYPTON MATRIX

J. CHEM. PHYS

V.80, P.3050-3. 1984.

JONES, LLWELLYN H. (INC-4)

SWANSON, BASIL I. (INC-4) 
$47-41$

PATTERNS OF ENERGY LEVELS AND SPECTRA FOR POLYATOMIC MOLECULES.

TIC,

1983. 28P. MN (LA-UR-84-0431)

HARTER, W. G.

47-42

PHASE DIAGRAM OF NITROGEN DETERMINED BY RAMAN SPECTROSCOPY FROM 15 TO 300

KAT PRESSURES TO 52 GPA.

J.PHYS. CHEM.,

V.88, P.2522-5. 1984.

BUCHSBAUM, STEVEN (P-10)

MILLS, ROBERT L. (P-10)

SCHIFERL, DAVID (M-6)

47-43

PLANE - WAVE BORN COLLISION STRENGTHS FOR ELECTRON - ION EXCITATIONCOMPARISON WITH OTHER THEORETICAL METHODS.

LOS ALAMOS, 1984.32P. (LA-10192-MS) CLARK, ROBERT EDWARD HOLMES $(x-7$ COLLINS, LEEA. $(T-4)$

47-44

POTENTIAL ENERGY SURFACE OF

CARBON 4 LTHIUM 4.

J. AM. CHEM. SOC.,

V.106, P.6246-9. 1984.

DISCH, RAYMOND L

SCHULMAN, JEROMEM.

RITCHIE, JAMES P. (T-14)

47-45

PREDISSOCIATION RATES AS A PROBE OF INTRAMOLECULAR DYNAMICS.

J. CHEM. PHYS.

V.80, P.3680-6. 1984

SUNDBERG, ROBERT L. (T-12)

HELLER, ERIC J. (T-12)

$47-46$

PROSPECTS FOR SINGLE - MOLECULE

DETECTION IN LIQUIDS BY LASER-

INDUCED FLUORESCENCE.

CONFERENCE ON ANALYTICAL CHEMISTAY

IN ENERGY TECHNOLOGY, 26TH, 1983,

KNOXVILLE. PROC., P.53-5. ELSEVIER,

1984.

ALSO PUBLISHED IN:

TIC,

1983. 4P. MN (LA-UR-83-3010)

TRKULA, MITCHELL (CHM-2)

DOVICHI, NORMAN J. (CNC-2)

MARTIN, JOHN C. (LS-4)

JETT, JAMES H. (LS-4)

KELLER, RICHARDA. (CHM-2)
47-47

REACTNE DIFFERENTIAL CROSS SECTIONS IN

THE ROTATING LINEAR MODEL - REACTIONS

OF FLUORINE ATOMS WITH HYDROGEN

MOLECULES AND THEIR ISOTOPIC VARIANTS.

J. PHYS. CHEM.

V.88, P.1194-9. 1984.

HAYES, EDWARD F.

WALKER, ROBERT B. (T-12)

$47-48$

REACTIVE RESONANCES AND ANGULAR

DISTRIBUTIONS IN THE ROTATING UNEAR

MODEL.

RESONANCES IN ELECTRON-MOLECULE

SCATTERING, 1984, ST. LOUIS

PROC., P.493-511. ACS, 1984.

ALSO PUBLISHED IN:

TIC,

1984. 44P. MN (LA-UR-84-1235)

HAYES, EDWARD F.

WALKER, ROBERT B. (T-12)

$47-49$

RESONANCE IONIZATION MASS SPECTROMETRY

AT LOS ALAMOS NATIONAL LABORATORY.

CONFERENCE ON ANALYTICAL CHEMISTRY

IN ENERGY TECHNOLOGY, 26TH, 1983,

KNOXVILLE. PROC., P.155-9. ELSEVIER,

1984.

ALSO PUBLISHED IN:

TIC,

1983. 7P. MN (LA-UR-83-3012)

NOGAR, NICHOLAS S. (CHM-2)

DOWNEY, STEPHEN W. (CHM-2)

KELLER, RICHARD A. (CHM-2)

MILLER, CHARLES M. (INC-7)

47-50

RESONANCE IONIZATION MASS SPECTROMETRY OF URANIUM WITH WNTRACAVITY LASER IONIZATION.

ANAL. CHEM.

V.56, P.827-8. 1984

DOWNEY, STEPHEN W. (CHM-2)

NOGAR, NICHOLAS S. (CHM-2)

MILER, CHARLES M. (INC-7)

47.51

RESONANCES IN ELECTRON - MOLECULE

SCATTERING AND PHOTOIONIZATION.

RESONANCES IN ELECTRON-MOLECULE

SCATTERING, 1984, ST. LOUIS.

PROC., P.65-86. ACS, 1984.

(541.2/R434EL/1984)

SCHNEIDER, BARRY I. (T-12)

COLUNS, LEE A. (T-4)

47-52

RESONANT MESONIC-MOLECULE FORMATION IN

MUON - CATALYZED DEUTERIUM - TRIITIUM

FUSION.

PHYS. REV. LETT.

V.52, P.605-8. 1984.

LEON, MELVIN (MP-3)
$47-53$

ROTATIONAL ENERGY SURFACES AND HIGH -

EIGENVALUE STRUCTURE OF POLYATOMIC

MOLECULES.

J. CHEM. PHYS.

V.80, P.4241-61. 1984.

HARTER, W.G.

PATTERSON, CHRIS W. (T-12)

47-54

SIZE CONSISTENCY IN PERTURBATION

THEORIES.

J. CHEM. PHYS.

V.80, P.1225-9. 1984

SHEPPARD, MAURICE G. (T-12)

47-55

SPECTRAL EMISSION FROM GLOW PEAKS IN

$X$-IRAADIATED ALMINUM OXIDE.

J. APPL. PHYS.,

V.55, P.3437-40. 1984.

COOKE, D.W.

47-56

SPECTROSCOPIC DIAGNOSTICS OF AN ARGON

PLASMA JET SEEDED WITH URANIUM.

CONFERENCE ON ANALYTICAL CHEMISTRY

IN ENERGY TECHNOLOGY, 2ND, 1983,

KNOXVILLE. PROC., P.333-5. ELSEVIER, 1984.

ANDERSON, GRAYDON K. (CHM-3)

47-67

SPECTROSCOPICEVIDENCE FOR DAYYDOV-

LIKE SOLITONS IN ACETANILIDE.

PHYS. REV.

V.30B, P.4689-702. 1984

CARERI, $G$.

BUONTEMPO. $U$.

GALLUZZI, $F$.

SCOTT, ALWYN C. (CNLS)

GRATTON, E.

SHYAMSUNDER, E.

47-58

SPIN-GLASS DYNAMICS DETERMINED FROM MUON SPIN RELAXATION AND NEUTRON SPIN ECHO MEASUREMENTS.

HYPERFINE INTERACT.

V.17-19, P.457-62. 1984.

ALSO PUBLISHED IN:

TIC.

1983. 10P. MN (LA-UR-83-1029)

HEFFNER, AOBERT H. (MP-3)

LEON, MELVIN (MP-3)

MACLAUGHLIN, DOUGLAS E

47-59

STRESSES AND ELASTIC CONSTANTS OF

METALS WITH CLASSICAL ION MOTION.

PHYS. REV.,

V.30A, P.616-8. 1984.

WAULACE, DUANE C. $(X-4)$

SCHIFERL, SHEILA K. $(\alpha-4)$

STRAUB, GALEN K. $(T-4)$ 
4750

STRUCTURE OF NITROGEN DEUTERIUM 3 SOLIDS I AND II AT HIGH PRESSURE BY NEUTRON DIFFRACTION

J. CHEM. PHYS.,

V.81, P.6034-7. 1984.

ECKERT, JUERGEN (P-8)

MILLS, ROBERT L. (P-10)

SATIJA, SUSHIL $K$.

47-61

TEMPERATURE AND HYDROGEN-CONCENTRATION DEPENDENCE OF HYDROGEN OPTIC VIBRATIONAL

FREQUENCIES IN ALPHA - AND ALPHA PRIME

PHASENIOBIUM HYDRIDE - STATIC LATTICE

STRAIN AND ANHARMONICITY EFFECTS.

PHYS. REV.

V.30B, P.4329-37. 1984.

TONKS, DAVIS L. (T-11)

47-62

TEMPERATURE DEPENDENCE OF HYDROGEN

STTE OCCUPANCY IN YTTRIUM DIHYDRIDE.

SOLID STATE COMMUN.,

V.49, P.475-8. 1984.

GOLDSTONE, JOYCE A. (P-8)

ECKERT, JUERGEN (P-B)

RICHARDS, PETER M.

VENTURINI, EUGENE L

\section{7-63}

TEMPERATURE DEPENDENCE OF THE RESONANCE RAMAN SPECTRA OF PLASTOCYANIN AND AZURIN BETWEEN CRYOGENIC AND AMBIENT CONDITIONS.

PROC. NATL. ACAD. SCI.,

V.81,P.1263-7. 1984

WOODRUFF, WILLIAMH.

NORTON, KENNETH A.

SWANSON, BASIL I. (INC-4)

FRY, HERBERT A. (INC-4)
47-64

TEMPERATURE INDEPENDENT SPHERICAL POTENTIAL WHICH REPRODUCES EXACTLY THE SECOND VIRIAL COEFFICIENT OF NONPOLAR MOLECULES.

J. CHEM. PHYS.,

V.81, P.3360-1. 1984

SHAW, MILTON S. (T-14)

JOHNSON, JAMES D. (T-4)

47-65

THE 3 NU 3 OVERTONE INTENSITIES AND

VIBRATIONAL ANHARMONICITY CONSTANTS OF URANIUM HEXAFLUORIDE AND PLUTONIUM HEXAFLUORIDE.

CHEM. PHYS. LETT.

V.104, P.79-82. 1984.

KIM, KYU C. (MST-11)

BRIESMEISTER, RICHARD A. (MST-11

CAMPBELL, GEORGE M. (MST-11)

PERSON, WILLIS B.

47-66

THEORETICAL STUDY OF DIMERIC FORMS OF GROUND - STATE BENZENE MOLECULES.

J. AM. CHEM. SOC.,

V.106, P.5439-46. 1984

ENGELKE, RAY P. (M-3)

HAY, P. JEFFREY (T-12)

KLEIER, DANIEL A. (T-12)

WADT, WILLARD R. (T-12)

47-67

THERMODYNAMICS OF DENSE FLUID NITROGEN

BY MOLECULAR DYNAMICS.

J. CHEM. PHYS.,

V.80,P.1279-93. 1984

JOHNSON, JAMES D. (T-4)

SHAW, MILTON S. (T-14)

HOLIAN, BRAD LEE $(T-4)$
$47-68$

USE OF SCALED ORBITAL FUNCTIONS IN

SELF - CONSISTENT FIELD CALCULATIONS FOR ATOMS.

INT. J. QUANTUM CHEM., SYMP.,

V.18, P.359-68. 1984.

LIBERMAN, DAVID A. (T-4)

47-69

VECTOR FIELDS, LINE INTEGRALS, AND THE HAMILTON - JACOBI EQUATION-

SEMICLASSICAL QUANTIZATION OF BOUND

STATES.

PHYS. REV.

V.30A, P.5-18. 1984.

DE LEON, NELSON (T-12)

HELLR, ERIC J. (T-12)

47-70

WAVEPACKET APPROACH TO GAS - SURFACE

SCATTERING - APPLICATION TO SURFACES WITH IMPERFECTIONS.

SURF. SCI.,

V.139, P.260-78. 1984.

DROLSHAGEN, GERHARD (T-12)

HELLER, ERIC J. (T-12)

47-71

$X$ - RAY ABSORPTION SPECTRA IN RAREEARTH AND URANIUM INTERMETALLICSLOCALIZED VERSUS ITINERANT FINAL

STATES.

PHYS. REV.,

V.29B, P.568-75. 1984.

LAWRENCE, J.M.

DENBOER, M. L

PARKS, RONALD D.

SMITH, JAMES L. (MST-5) 
48. PHYSICS-COSMIC AND TERRESTRIAL

48-1

"DRIFT DISSIPATIVE INSTABIUTY"-

A CRITIQUE.

J. ATMOS. TERR. PHYS.

V.46, P.685-7. 1984.

GARY, S. PETER (ESS-8)

48-2

ANALYSIS OF MAGNESIUM X1 LINE PROFILES FROM SOLAR ACTIVE REGIONS.

ASTROPHYS.J.,

V.282, P.784-92. 1984.

BLAKE, RICHARD L (P-4)

COWAN, ROBERT D. (T-4)

FELTHAUSER, HARAY E. (P-4)

FENIMORE, EDWARDE. (ESS-9)

HOCKADAY, MARY P. (P-14)

BELY-DUBAU, FRANCOISE

FAUCHER, PAUL

STEENMAN-CLARK, LOIS

48-3

APPLICATION OF ATMOSPHERIC TRANSPORT MODELS FOR COMPLEX TERRAIN.

J. CLIM. APPL. METEOROL.

V.23, P.240-6. 1984.

KING, DAVID S. $(x-5)$

BUNKER, SUSAN S. (X-5)

48-4

ASSOCIATIONS OF GEOMAGNETIC ACTIVITY

WITH PLASMA SHEET THINNING AND

EXPANSION - A STATISTICAL STUDY.

J. GEOPHYS. RES.,

V.89A, P.5471-8. 1984.

HONES, EDWARD W., JR. (ESS-8)

PYTTE, T.

WEST, HARRY I., JR.

48-5

ATMOSPHERIC TRANSPORT MODELS FOR COMPLEX TERRAIN.

J. CLIM. APPL METEOROL.

V.23, P.235-7. 1984.

DAVIS, CECIL G. (X-5)

BUNKER, SUSAN S. $(X-5)$

MUTSCHLECNER, J. PAUL $(X-5)$

$48-6$

CEPHEID EVOLUTION.

TIC,

1984. 23P. MN (LA-UR-84-1996)

BECKER, STEPHEN A. (X-2)

48-7

CHEMISTRY AND DYNAMICS OF SULFUR

HEXAFLUOAIDE INUECTIONS INTO THE F

RECION.

J. GEOPHYS. RES.

V.89A, P.3929-36. 1984

BERNHARDT, PAUL A. (ESS-7)
48-8

CLASSICAL KALUZA - KLEIN COSMOLOGY FOR A TORUS SPACE WITH A COSMOLOGICAL

CONSTANT AND MATTER.

PHYS. REV.

V.30D, P.2054-60. 1984.

TOSA, YASUNARI (T-8)

48-9

CLOSE ENCOUNTERS BETWEEN A STAR-

PLANET SYSTEM AND A STELLAR INTRUDER.

ASTRON. J.

V.89, P.1559-64. 1984.

HILLS, JACK G. (T-6)

48-10

COUECTIVE CAPTURE OF RELEASED UTTHIUM

IONS IN THE SOLAR WIND.

J. GEOPHYS. RES.,

V.89A, P.7327-30. 1984.

WINSKE, DAN

WU, C.S.

U,Y.Y.

ZHOU, G.C.

48-11

COLHDING WINDS - INTERACTION REGIONS

WITH STRONG HEAT CONDUCTION.

ASTROPHYS. J.

V.280,P.313-8. 1984.

IMAMURA, JAMES N. (ESS-9)

CHEVALIER, ROGER A.

48-12

COLLISIONLESS DISSIPATION IN

QUASI - PERPENDICULAR MAGNETIC SHOCKS.

J. GEOPHYS. RES.

V.89A, P 2142-50. 1984.

FORSLUND, DAVID W. $(X$

QUEST, KEVIN B. $(X-1)$

BRACKBILL, JEREMIAH U. $(X-1)$

LEE, KENNETH $(\alpha-1)$

48-13

COMPARISON OF THE X-RAY OBSERVATIONS

OF THE AM HERCULIS OBJECTS TO THEORY.

ASTROPHYS. J.

V.285, P.223-31. 1984

IMAMURA, JAMES N. (ESS- $\theta$ )

48-14

COMPUTER SIMULATION OF RECONNECTION IN

PLANETARY MAGNETOSPHERES.

TIC.

1983. 17P. MN (LA-UR-83-2515) BIRN, J.

48-15

CONSTRAINTS ON STELLAR EVOLUTION FROM

PULSATIONS.

TIC,

1983. 20P. MN (LA-UR-83-3215)

COX, ARTHUR N. (T-6)
48-16

COOKBOOK FOR DETERMINING ESSENTIAL

TRANSMISSION CHARACTERISTICS OF

SPHERICAL SECTION ELECTROSTATIC

ANALYZERS.

LOS ALAMOS

1984. 154P. (LA-10147-M)

GOSLING, JOHN T. (ESS-8)

THOMSEN, MICHELLE F. (ESS-8)

ANDERSON, RICHARD C. (ESS-8)

48-17

CORRELATED DYNAMICAL CHANGES IN THE NEAR - EARTH AND DISTANT MAGNETOTAIL

REGIONS - ISEE 3.

J. GEOPHYS. RES.

V.89A, P.3855-64. 1984.

BAKER, DANIEL N. (ESS-8)

BAME, SAMUEL J., JR. (ESS-8)

BELIAN, RICHARD D. (ESS-8)

FELDMAN, WILLIAM C. (ESS-8)

GOSLING, JOHN T. (ESS-8)

HIGBIE, PAUL R. (ESS-8)

HONES, EDWARD W., JR. (ESS-8)

MC COMAS, DAVID J. (ESS-8)

ZWICKL, RONALD D. (ESS-8)

48-18

CORRELATION BETWEEN THE HELIUM-

HYDROGEN RATIOS IN UPSTREAM PARTICLE EVENTS AND IN THE SOLAR WIND.

J. GEOPHYS. RES.,

V.89, P.1501-7. 1984.

IPAVICH, FRED $M$.

GOSLING, JOHN T. (ESS-8)

SCHOLER, M.

48-19

CYLTRAN CALCULATIONS OF ELECTRON EFFECTS ON XD DETECTORS.

LOS ALAMOS.

1984. 10P. (LA-10264-MS)

MAYER, STUART

BERZINS, GEORGE J. (ESS-9)

48-20

DENSITY SPECTRA AS FUNCTION OF

ALTITUDE IN AN IONSPHERIC BARIUM

RELEASE.

GEOPHYS. RES. LETT.

V.11, P.591-4, 1984.

LEBEDA, CHARLES F

GARY, S. PETER (ESS-8)

PONGRATZ, MORAIS B. (ESS-B) 
48-21

DETAILED EXAMINATION OF A PLASMOID IN THE DISTANT MAGNETOTAIL WITH ISEE 3.

GEOPHYS. RES. LETT.

V.11, P.1046-9. 1984

HONES, EDWARD W., JR. (ESS-B)

BIRN, J.

BAKER, DANIEL N. (ESS-8)

BAME, SAMUEL J., JR. (ESS-8)

FELDMAN, WILLIAM C. (ESS-8)

MC COMAS, DAVID J. (ESS-8)

ZWICKL, RONALD D. (ESS-8)

SLAVIN, J. A.

SMITH, E.J.

TSURUTANI, B.T.

48-22

DID THE BINARY STARS IN GLOBULAR CLUSTERS COME TO AN EARLY END BY BEING TOO ECCENTRIC?

ASTRON.J.

V.89, P.1811-4. 1984. HILLS, JACK G. (T-6)

48-23

DIGITAL HF RADAR OBSERVATIONS OF EQUATORIAL SPREAD - F.

TIC.

1984. 35P. MN (LA-UR-84-0264)

ARGO, PAUL E. (ESS-7)

48-24

OIMENSIONAL REDUCTION IN THE EARLY UNIVERSE - WHERE HAVE THE MASSIVE

PARTICLES GONE?

PHYS. LETT.

V.135B, P.378-82. 1984

KOLB, EDWARD W., JR. (T-6)

SLANSKY, RICHARD C. (T-8)

48-25

DIRECT OBSERVATIONS OF PASSAGES OF THE

DISTANCE NEUTRAL LINE ( 80 - 140 R E)

FOLLOWING SUBSTORM ONSETS - ISEE -3.

GEOPHYS. RES. LETT.

V.11,P.1042-5.1884

BAKER, DANIEL N. (ESS-8)

BAME, SAMUEL J., JR. (ESS-8)

BIRN, J.

FELDMAN, WILLIAM C. (ESS-8)

GOSUNG, JOHN T. (ESS-8)

HONES, EDWARD W., JR. (ESS-8)

ZWICKL, RONALD D. (ESS-8)

SLAVIN, J. A.

SMITH, E. J.

TSURUTANI, B.T

SIBECK, D. G.

48-26

DISCOVERY OF A 176 DAY PERIOD IN

$4 U 1820-30$.

ASTROPHYS.J.

V.284, P.L17-20. 1984

PAIEDHORSKY, WILLIAM C. (ESS-9)

TERRELL, NELSON JAMES (ESS-9)
48-27

DISCREPANCY IN THE CNO OPACITY BUMP

RESOLVED.

ASTROPHYS. $J$.

V.283, P.466-8. 1984

CARSON, T. R.

HUEBNER, WALTER F. $(T-4)$

MAGEE, NORMAN H. $(T-4)$

MERTS, ATHEL L. (T-4)

48-28

DOUBLE - MODE PULSATION.

PULSATIONS IN CLASSICAL AND

CATACLYSMIC VARIABLE STARS, 1882

BOULDER. PROC., P.157-69. JOINT INST.

LAB. ASTROPHYS., 1982

(523.844/P982CL/1982)

ALSO PUBLISHED IN.

TIC.

1982. 14P. MN (LA-UR-82-2304)

COX. ARTHUR N. (T-6)

48-29

DYNAMICAL CONSTRAINTS ON THE MASS AND

PERIHELION DISTANCE OF NEMESIS AND THE

STABILTY OF ITS ORBIT.

NATURE,

V.311, P.636-8. 1984.

HILLS, JACK G. (T-6)

$48-30$

ELECTROMAGNETIC ION BEAM INSTABILTIES.

PHYS. FLUIDS,

V.27,P.1852-61. 1884.

GAFY, S. PETER (ESS-8)

SMITH, CARL W.

LEE, M. A.

GOLDSTEIN, MELWNL

FORSLUND, DAVID W. $(X-1)$

48-31

ELECTRON HEATING AT INTERPLANETARY

SHOCKS.

SOLAR WIND 5, 1982, WOODSTOCK.

PROC., P.403-11, 1983. (NASA-CP-2280)

ALSO PUBLISHED IN:

TIC.

1982. 23P. MN (LA-UR-83-0259)

FELDMAN, WILLIAM C. (ESS-8)

ASBRIDGE, JOHN R. (ESS-8)

BAME, SAMUEL J., JR. (ESS-8)

GOSLING, JOHN T. (ESS-8)

ZWICKL, RONALD D. (ESS-8)

48-32

ELECTRON VELOCITY DISTRIBUTIONS NEAR

INTERPLANETARY SHOCKS.

J. GEOPHYS. RES.

V.88, P.9949-58. 1983.

FELDMAN, WILLIAM C. (ESS-8)

ANDERSON, RICHARD C. (ESS-8)

BAME, SAMUEL J., JR. (ESS-8)

GOSUNG, JOHN T. (ESS-B)

ZWICKL, RONALD D. (ESS-8)

SMITH, E.J.
$48-33$

ELECTRON VELOCITY DISTRIBUTIONS NEAR COLLISIONLESS SHOCKS

TIC.

1984. 40P. MN (LA-UR-84-1199)

FELDMAN, WLLLIAM C. (ESS-8)

48-34

ELECTROSTATIC HISS AND THE BEAM DRIVEN

ELECTRON ACOUSTIC INSTABILITY IN THE

DAYSIDE POLAR CUSP.

GEOPHYS. RES. LETT.

V.11,P.1180-3. 1984.

TOKAR, ROBERT L. (ESS-8)

GARY, S. PETER (ESS-8)

48-35

ENERGETIC PARTICLE TRANSPORT IN THE UPSTREAM REGION OF JUPITER - VOYAGER RESULTS.

J. GEOPHYS. RES.,

V.89A, P.3775-87. 1984 .

BAKER, DANIEL N. (ESS-8)

ZWICKL, RONALD D. (ESS-8)

KRIMIGIS, S. M.

CARBARY, J.F.

ACUNA, M. H.

48-36

ENERGY OISTRIBUTIONS AND YIELDS OF

SECONDARY IONS AND ELECTRONS EMITTED

BY GIOTTO DURING HALLEY ENCOUNTER.

TIC.

1983. 9P. MN (LA-UR-63-2887)

YOUNG, DAVID T. (ESS-8)

48-37

EVIDENCE FOR SLOW - MODE SHOCKS IN THE

DEEP GEOMAGNETIC TAIL.

GEOPHYS. RES. LETT.

V.11, P.599-602, 1984 .

FELDMAN, WILLIAM C. (ESS-8)

SCHWARTZ, STEVEN J.

BAME, SAMUEL J., JR. (ESS-8)

BAKER, DANIEL N. (ESS-8)

BIRN, J.

GOSLING, JOHN T. (ESS-8)

HONES, EDWARD W., JR. (ESS-8)

MC COMAS, DAVID J. (ESS-8)

SLAVIN, J. A.

SMITH, E.J.

ZWICKL, RONALD D. (ESS-8)

48-39

EVOLUTION OF ION DISTRIBUTIONS ACROSS

THE NEARLY PERPENDICULAR BOW SHOCK-

SPECULARLY AND NON-SPECULARLY REFLECTED-

GYRATING IONS.

J. GEOPHYS. RES.

V.88, P.6121.36. 1983

SCKOPKE, $N$.

PASCHMANN, GOTZ

BAME, SAMUEL J., JR. (ESS-8)

GOSUNG, JOHN T. (ESS-8)

RUSSELL, C.T. 
48-40

EVOLUTION OF STRUCTURE IN THE PLACES (POSITION LOCATION AND COMMUNICATION EFFECTS SIMULATIONS) BARIUM CLOUDS.

LOS ALAMOS.

1984. 177P. (LA-09648-MS)

SIMONS, DAVD J. (ESS-7)

LEBEDA, CHARLES F.

PONGRATZ, MORRIS B. (ESS-8)

FITZGERALD, THOMAS JOSEPH

DUPRE, ROBERT $R$.

48-41

EVOLUTION OF THE EARTH'S DISTANT MAGNETOTAIL - ISEE 3 ELECTRON PLASMA RESULTS.

J. GEOPHYS. RES.

V.89, P.11,007-12. 1984.

ZWICKL, RONALD.D. (ESS-8)

BAKER, DANIEL N. (ESS-8)

BAME, SAMUEL J., JR. (ESS-8)

FELDMAN, WILLIAMC. (ESS-8)

GOSLING, JOHN T. (ESS-8)

HONES, EDWARD W., JA. (ESS-8)

MC COMAS, DAVID J. (ESS-8)

TSURUTANI, B.T.

SLAVIN, J. A.

48-42

EXOSAT - EUROPE'S NEW X - RAY

SATELLITE.

SKY TELESCOPE,

MAY, P.397-401. 1984.

CORDOVA, FRANCE ANNE (ESS-9) MASON, K. O.

$48-43$

EXTENSION OF THE RECONNECTION THEORY

OF TWO - RIBBON SOLAR FLARES.

SOL. PHYS.,

V.93,P.352-61. 1984.

KOPP, ROGER ALAN $(X-1)$

POLETTO, G.

$48-44$

FIRM BOUNDS ON THE NEUTRINO MASS FROM THE DISTRIBUTION OF DARK MATTER IN

GALAXIES.

ASTROPHYS. J.,

V.282, P.11-8. 1984.

MADSEN, J.

EPSTEIN, RICHARD I. (ESS-9)

48-45

FIRST - FLIGHT ESCAPE FROM SPHERES

WITH R MINUS 2 DENSITY DISTRIBUTION.

ASTRON. ASTROPHYS.

V.135, P.177-80. 1984.

HUEBNEA, WALTER F. $(T-4)$

KEADY, JOHN J. (T-4)

48-46

FLAT ROTATION CURVES ACCORDING TO THE FRAGMENTATION/SHEAR FLOW MODEL OF

GALAXY FORMATION.

ASTROPHYS: SPACE SCl.

V.105, P.109-29. 1984.

BAOWN, WILBUR K. (P-15)
48-47

GALAXIES FROM POISSON FLUCTUATIONS.

ASTROPHYS. J.

V.281, P.545-53. 1984.

EPSTEIN, RICHARD I. (ESS-9)

48-48

GAMMA BURST EMISSION FROM NEUTRON

STAR ACCRETION.

HIGH ENERGY TRANSIENTS IN

ASTROPHYSICS, 1983, SANTA CRUZ.

PROC., P.548-54. AIP, 1984

(523.015/H638EN/1983)

ALSO PUBLISHED IN:

TIC.

1983. 8P. MN (LA-UR-83-2586)

COLGATE, STIRLING A. $(T-6)$

PETSCHEK, ALBERT G. (P-DO)

SARRACINO, ROBERT

48-49

GAMMA BURSTS.

NUMERICAL ASTROPHYSICS, 1982,

UNIV. OF ILL. PROC., P.17t-4.

JONES \& BARTLETT PUBL., 1982.

(523.01/N971AS/1982)

ALSO PUBLISHED IN.

TIC,

1982. 6P. MN (LA-UR-B2-3596)

COLGATE, STIRLING A. (T-6)

PETSCHEK, ALBERT G. (P-DO)

48-50

HIGH-ENERGY PROTON DRIFT ECHOES-

MULTIPLE PEAK STRUCTURE.

J. GEOPHYS. RES.,

V.89, P.9101-6. 1984.

BELIAN, RICHARD D. (ESS-8)

BAKER, DANIEL N. (ESS-B)

HONES, EDWARD W., JR. (ESS-8)

HIGEIE, PAUL R. (ESS-8)

48-51

HISTORY OF X - RAY TRANSIENTS AS SEEN

BY VELA, 1969 - 1979.

ANN. N. Y. ACAD. SCl.,

V.422, P.282-3. 1984.

TERRELL, NELSON JAMES (ESS-9)

PRIEDHORSKY, WILLIAM C. (ESS-9)

BELIAN, RICHARD D. (ESS-8)

CONNER, JERRY P. (ESS-DO)

EVANS, W. DOYLE (ESS-9)

48-52

HRTS II EUV OBSERVATIONS OF A SOLAR

EPHEMERAL REGION

ASTROPHYS. $J$

V.278, P.428-40. 1984. ROUSSEL-DUPRE, ROBERT (ESS-7)

WRATHALL, JAY

NICOLAS, K.R.

BARTOE, J.O.F.

BRUECKNER, G. E.
48-53

IMPLCATIONS OF THE 1100 UT MARCH 22,

1979 CDAW 6 SUBSTORM EVENT FOR THE ROLE OF MAGNETIC RECONNECTION IN THE GEOMAGNETIC TAIL.

MAGNETIC CONNECTION IN SPACE AND

LABORATORY PLASMAS, 1983, LOS ALAMOS

PROC., P.203-7. AGU, 1984.

(538.766/M196RE/1983)

ALSO PUBLISHED IN:

TIC.

1983. 18P. MN (LA-UR-83-3498)

FRITZ, T. A. (ESS-8)

BAKER, DANIEL N. (ESS-8)

MC PHERRON, R. L

LENNARTSSON, $W$.

48-54

INTERACTION OF COSMIC RAY SECONDARIES IN EXTRATERRESTRIAL MATTER - SIMULATIONS WITH HIGH ENERGY PROTONS AND NEUTRONS.

TERRA COGNITA,

V.3, P.74. 1983.

ENGLERT, $P$.

THEIS, $S$.

REEDY, ROBERT C. (INC-11)

ARNOLD, JAMES R.

48-55

INVERSE COMPTONIZATION VERSUS THERMAL

SYNCHROTRON

HIGH ENERGY TRANSIENTS IN

ASTROPHYSICS, 1983, SANTA CRUZ.

PROC., P.590-6. AIP, 1984

(523.015/H638EN/1983)

ALSO PUBLISHED IN:

TIC,

1983. 7P. MN (LA-UA-83-2844)

FENIMORE, EDWARD E. (ESS-9)

KLEBESADEL, RAY W. (ESS-9)

LAROS, JOHN G. (ESS-9)

48-56

INVESTIGATION OF LAMINAR SHOCKS

TIC,

1984. 5P. MN (LA-UR-64-0892)

LEE, KENNETH $(X-1)$

ALDAICH, CHARLES H. (X-1)

BAME, SAMUEL J., JR. (ESS-8)

FORSLUND, DAVID W. (X-1)

GARY, S. PETER (ESS-8)

GOSLING, JOHN T. (ESS-6)

QUEST, KEVIN B. $(X-1)$

THOMSEN, MICHELLE F. (ESS-8)

WINSKE, DAN $(\mathrm{X}-1)$

MELOT, $M$.

48-57

ION REFLECTION, GYRATION, AND

DISSIPATION AT SUPERCRITICAL SHOCKS

TIC.

1984. 35P. MN (LA-UR-84-1391)

GOSLNG, JOHN T. (ESS-8)

ROBSON, A.E. 
$48-68$

IRON CORE COLLAPSE MODELS OF TVPE II

SUPERNOVAE.

NUMERICAL ASTROPHYSICS, 1982.

UNIV. OF ILL. PROC., P.403-21.

JONES \& BARTLETT PUBL., 1982.

(523.01/N971AS/1982)

BOWERS, RICHARD L. (X-2)

48-59

IS THE METAL CONTRIBUTION TO THE

ASTROPHYSICAL OPACITY INCORRECT?

ASTROPHYS. J.,

V.283, P.264-72. 1984

MAGEE, NORMAN H. $(T-4)$

MERTS, ATHEL L. (T-4)

HUEBNER, WALTER F. $(T-4)$

$48-60$

ISEE - 3 PLASMA MEASUREMENTS IN THE LOBES OF THE DISTANT GEOMAGNETIC TAILINFERENCES CONCERNING RECONNECTION AT THE DAYSIDE MAGNETOPAUSE.

MAGNETIC RECONNECTION IN SPACE AND LABORATORY PLASMAS, 1983, LOS ALAMOS. PROC., 1 P. AGU, 1984. COSLING, JOHNT. (ESS-8)

\section{8-61}

ISEE - 3 PLASMA MEASUREMENTS IN THE LOBE OF THE DISTANT GEOMAGNETIC TAILINFERENCES CONCERNING RECONNECTION AT THE DAYSIDE MAGNETOPAUSE.

MAGNETIC RECONNECTION IN SPACE AND LABORATORY PLASMAS, E.W. HONES, JR., ED., P.157-8. AGU, 1984. (538.766/M196RE/1983)

GOSLING, JOHNT. (ESS-8)

48-62

JOINT TIMING ANALYSIS OF A0535+26 AT 1980 OUTBURST.

HIGH ENERGY TRANSIENTS IN

ASTROPHYSICS, 1983, SANTA CRUZ

PROC., P.146-9. AIP, 1984.

(523.015/H638EN/1983)

ALSO PUBLISHED IN:

TIC,

1983. 5P. MN (LA-UR-83-2681)

KAWAI, N. (ESS-9)

NAGASE, F.

48-63

JOURNEYS OF A SPACECRAFT.

LOS ALAMOS SCI.,

SPRING, NO.10, P.33-55. 1984.

GOSLING, JOHN T. (ESS-8)

BAKER, DANIEL N. (ESS-8)

HONES, EDWARD W., JR. (ESS-8)

48-64

IINETIC THEORY OF CURRENT AND DENSITY

JRIFT IN INSTABILITIES WITH WEAK

'HARGED - NEUTRAL COLLISIONS.

J. GEOPHYS. RES.

V.89A, P.179-86. 1984.

GARY, S. PETER (ESS-8)
48-65

LABORATORY SIMULATION OF UNMAGNETIZED SUPERNOVA REMNANTS - ABSENCE OF A BLAST WAVE.

ASTROPHYS. J.,

V.280, P.802-8. 1984.

BOROVSKY, JOSEPH E. (ESS-8)

PONGRATZ, MORRIS B. (ESS-8)

ROUSSEL-DUPRE, ROBERT (ESS-7)

TAN, TAI HO (P-4)

48-66

LOG N-LOG S IS INCONCLUSIVE.

HIGH ENERGY TRANSIENTS IN

ASTROPHYSICS, 1983, SANTA CRUZ

PROC. P.429-33. AIP, 1984.

(523.015/H638EN/1983)

ALSO PUBLISHED IN.

TIC,

1983. 6P. MN (LA-UR-83-2776)

KLEBESADEL, RAY W. (ESS- 9 )

FENIMORE, EDWARD E. (ESS-9)

LAROS, JOHN G. (ESS-9)

48-67

LONG-TERM OBSERVATIONS OF X-RAY

SOURCES - THE AQUILA - SERPENS-

SCUTUM REGION

ASTROPHYS. $\mathrm{J}$.

V.28D, P.661-70. 1984

PRIEDHORSKY, WILLIAM C. (ESS-9)

TERREL, NELSON JAMES (ESS-9)

48-68

MICROINSTABILITIES IN THE HIGH

LATITUDE F REGION - A BRIEF REVIEW.

TIC.

1983. 32P. MN (LA-UR-83-2997)

GARY, S. PETER (ESS-8)

48-69

MICROPROBE ANALYSES OF RARE EARTH

ELEMENT FRACTIONATION IN METEORITIC MINERALS.

NUCL. INSTRUM. METHODS,

V.B3, P.677-80. 1984.

ALSO PUBLISHED IN:

TIC,

1983. 18P. MN (LA-UR-83-2368)

BENJAMIN, TIMOTHY M. (INC-7)

DUFFY, CLARENCE J. (INC-11)

MAGGIORE, CARL J. (E-11)

ROGERS, PAMELA S. Z. (INC-7)

WOOLUM, D. S.

BURNETT, D. $\mathrm{S}$.

MURRELL; M.T.

48-70

MICROTHEORY OF COLLISIONLESS SHOCK

CURRENT LAYERS

TIC.

1984. 43P. MN (LA-UR-84-0512) WINSKE, DAN
48-71

MULTIFREQUENCY OBSERVATIONS OF THE BL

LACERTAE OBJECTS OQ 530 AND ON 325.

ASTROPHYS. J.

V.284, P.512-8. 1984.

WORRALL, D. M.

PUSCHELL, J.J.

BRUHWEILER, F.C.

SITKO, M. L.

STEIN, $W: A$.

ALLER, M. F.

ALLER, H. D.

HODGE, P. E

RUDY.R.J.

MILLER, H. R.

WISNIEWSKI, W. Z.

CORDOVA, FRANCE ANNE (ESS-9)

MASON, K. $O$

48-72

MULTIPLE SPACECRAFT OBSERVATIONS OF

INTERPLANETARY SHOCKS - ISEE THREE-

DIMENSIONAL PLASMA MEASUREMENTS.

J. GEOPHYS. RES.,

V.88, P.9941-7. 1983

AUSSELL, C. T.

GOSLING, JOHNT. (ESS-8)

ZWICKL, RONALD D. (ESS-8)

SMITH, E. J.

48-73

MULTIPLE SPACECRAFT OBSERVATIONS OF INTERPLANETARY SHOCKS - CHARACTERISTICS OF THE UPSTREAM ULF TURBULENCE.

SOLAR WIND 5, 1982, WOODSTOCK.

PROC., P.385-99. 1983. (NASA-CP-2280)

ALSO PUBLSHED IN:

TIC,

1982. 17P. MN (LA-UR-83-1172)

RUSSELL, C. T

SMITH, E. J.

TSURUTANI, B.T.

GOSLING, JOHN T. (ESS-8)

BAME, SAMUEL J., JR. (ESS-8)

48-74

NASA DATA SYSTEMS USERS WORKING GROUP-

RECOMMENDATIONS FOR IMPROVED SCIENTIFIC INTERACTIONS.

EOS,

V.65, NO.6, P.46. 1984

BAKER, DANIEL N. (ESS-8)

ZWICKL, RONALD D. (ESS-8)

GREEN, J. L.

48-75

NEW OBSERVATION OF TWOHOLE CORE-

LEVEL SATELLITES IN COPPER, SILVER,

AND GOLD.

PHYS. REV.

V.29B, P.4800-2. 1984.

MARTENSSON, NILS

NYHOLM, RALF

JOHANSSON, BORJE 
48-76

NUCLEAR PHYSICS PROBLEMS FOR ACCRETING NEUTRON STARS

TIC,

1983. 7P. MN (LA-UR-83-2486)

WALLACE, RICHARD K. (X-2)

WOOSLEY, S. E.

48-77

NUMERICAL SIMULATIONS OF CONVECTIVELY EXCITED GRAVITY WAVES.

TIC,

1983. 11P. MN (LA-UR-83-2896)

GLATZMAIER, GARY ANDREW (T-6)

48-78

NUMERICAL SIMULATIONS OF STELLAR CONVECTIVE DYNAMOS - 1. THE MODEL AND METHOD.

J. COMPUT. PHYS.

V.55, P.461-83. 1984

GLATZMAIER, GARY ANDREW (T-6)

48-79

OBSERVATIONS AND A NEW INTERPRETATION

FOR THE DELTA SCUTI VARIABLE STAR VZ

CANCRI.

ASTROPHYS. $J$

V.284,P.250-6. 1984.

COX, ARTHUR N. (T-6)

MCNAMARA, BERNARD J.

RYAN, WILLIAM

48-80

OBSERVATIONS OF AN IONOSPHERIC

PERTURBATION ARISING FROM THE COALINGA

EARTHQUAKE OF MAY 2, 1983.

J. GEOPHYS. RES.,

V.89A, P.6835-9. 1984

WOLCOTT, JOHN H. (ESS-7)

SIMONS, DAVID J. (ESS-7)

LEE, D. D

NELSON, R. A.

48-81

OBSERVATIONS OF QUASI - COHERENT SOFT

$X$ - RAY OSCILLATIONS IN U GEMINORUM

AND SS CYGNI.

ASTROPHYS. J.

V.278, P.739-53. 1984.

CORDOVA, FRANCE ANNE (ESS-9)

CHESTER, T. J.

MASON, K.O

KAHN, S. M.

GARMIRE, G.P.

48-82

OBSERVATIONS OF THE INTERSTELLAR ICE

GRAIN FEATURE IN THE TAURUS MOLECULAR

CLOUDS.

TIC,

1983. 9P. MN (LA-UR-B3-3621)

WHITTET, D. C. B.

BODE, M. F. (ESS-9)

LONGMORE, A. J

BAINES, D.W.T.

EVANS, $A$.
48-83

OBSERVATORY REPORT.

BULL. AM. ASTRON. SOC.

V.16, P.185-93. 1984.

COX, ARTHUR N. (T-6)

HILLS, JACK G. (T-6)

48-84

ON THE PRODUCTION OF TECHNETIUM IN

THERMALLY PULSING RED GIANT STARS.

ASTROPHYS. $J$.

V.283, P.313-5. 1984.

COSNER, KENNETH R.

DESPAIN, KEITH HOWARD (X-2)

TRURAN, JAMES W.

48-85

PARTICLE AND FIELD SIGNATURES OF

SUBSTORMS IN THE NEAR MAGNETOTAIL

MAGNETIC RECONNECTION IN SPACE AND

LABORATORY PLASMAS, 1983, LOS ALAMOS.

PROC., P.193-201. AGU, 1984

(538.766/M196RE/1983)

ALSO PUBLISHED IN

TIC.

1983. 44P. MN (LA-UR-83-3239)

BAKER, DANIEL N. (ESS-8)

48-86

PHASE SEPARATION IN A CHONDRULE FRAGMENT

FROM THE PIANCALDOL (LL3) CHONDRITE.

CHONDRULES AND THEIR ORIGINS

E.A. KING, ED., P.235-41. LPI, 1983.

PLANNER, H. N. (ESS-2)

48-87

PLASMA ENTRY INTO THE DISTANT TAIL LOBES

ISEE - 3 .

GEOPHYS. RES. LETT.

V.11, P.1078-81. 1984.

GOSLING, JOHN T. (ESS-8)

BAKER, DANIEL N. (ESS-8)

BAME, SAMUEL J., JR. (ESS-8)

HONES, EDWARD W., JR. (ESS-8)

MC COMAS, DAVID J. (ESS-8)

ZWICKL, RONALD D. (ESS-8)

SLAVIN, J. A.

SMITH, E. J.

TSURUTANI, B. T.

48-88

PLASMA PROPERTIES OF DRIVER GAS

FOLLOWING INTERPLANETARY SHOCKS OBSERVED

BY ISEE-3.

SOLAF WIND 5, 1982, WOODSTOCK.

PROC., P.711-7. 1983. (NASA-CP-2280)

ALSO PUBLISHED IN:

TIC

1982. 14P. MN (LA-UR-83-0348)

ZWICKL, RONALD D. (ESS-8)

ASBRIDGE, JOHN R. (ESS-8)

BAME, SAMUEL J., JR. (ESS-8)

FELDMAN, WILLIAM C. (ESS-8)

GOSLING, JOHN T. (ESS-8)

SMITH, E. J.
48-89

PLASMA SHEET BEHAVIOR DURING SUBSTORMS MAGNETIC RECONNECTION IN SPACE AND LABORATORY PLASMAS, 1983, LOS ALAMOS.

PROC., P.178-84. AGU, 1984.

(538.766/M196RE/1983)

ALSO PUBLISHED IN

TIC.

1983. 30P. MN (LA-UR-83-3361)

HONES, EDWARD W., JR. (ESS-8)

$48-90$

POPULATION II CEPHEIDS

PUBL ASTRON. SOC. PAC.

V.96, P.677-91. 1984.

WALLERSTEIN, GEORGE

COX, ARTHUR N. (T-6)

48-91

POWER DISSIPATION AT SLOW - MODE SHOCKS

IN THE DISTANT GEOMAGNETIC TAIL.

GEOPHYS. RES. LETT.

V.11, P.1058-61. 1984.

FELDMAN, WLLLIAM C. (ESS-8)

BAKER, DANIEL N. (ESS-8)

BAME, SAMUEL J., JR. (ESS-8)

BIRN, J.

HONES, EDWARD W., JR. (ESS-8)

SCHWARTZ, STEVEN J.

TOKAR, ROBERT L (ESS-8)

48-92

PREDICTION OF NONLINEAR STRUCTURAL RESPONSE IN LMFBR ELEVATED - TEMPERATURE PIPING. LOS ALAMOS

1984. 22P. (LA-10090-MS)

FARRAR, CHARLES R (Q-13)

48-93

RADIATIVELY DRIVEN DUST - BOUNDEO IMPLOSIONS - FORMATION AND STABILITY OF DENSE GLOBULES.

ASTROPHYS. J.,

V.282, P.178-90. 1984.

SANDFORD, MAXWELL T. (ESS-5)

WHITAKER, RODNEY W. (ESS-5)

KLEIN, RICHARD I.

48-94

RELATIVISTIC CHROMOHYDRODYNAMICS AND

YANG-MILLS VLASOV PLASMA.

PHYS. LETT.,

V.105A, P.225-8. 1984.

HOLM, DARRYL D. (CNLS)

KUPERSHMIDT, BORIS ABAAM (CNLS)

48-95

REPLY (TO RICE ON THE STABILITY OF

SHEAR CRACKS AND THE ...)

J. GEOPHYS. RES.,

V.89B, P.2508. 1984

DIENES, JOHN K. (T-3)

48-96

REPORT OF THE LUNAR BASE WORKING GROUP APRIL 23 - 27, 1984.

LOS ALAMOS

1984. 41P. (LALP-84-043)

DUKE, MICHAEL B

MENDELL, WENDELL W.

KEATON, P. W., JR. (ADPA) 
48-97

REPORT ON THE CHAPMAN CONFERENCE ON MAGNETIC RECONNECTION.

EOS,

V.65, NO.13, P.124-5. 1984.

HONES, EOWARD W., JR. (ESS-8)

$48-98$

REVIEW OF ASTROPHYSICAL RESEARCHAT

LOS ALAMOS.

TiC.

1883. 7P. MN (LA-UR-83-2329)

KIRKPATRICK, RONALD C. (X-2)

48-99

REVIEW OF RADIO - FREQUENCY, NONLINEAR

EFFECTS ON THE IONOSPHERE.

TIC,

1983. 13P. MN (LA-UR-83-3368)

GORDON, WILLIAM E.

DUNCAN, LEWIS M. (ESS-7)

48-100

ROLE OF THE RADIATION PRESSURE

GRADIENT IN GIANT AND SUPERGIANT

STAREVOLUTION.

TIC.

1983. 4P. MN (LA-UR-83-2969)

BRUNISH, WENDEE M. (T-6)

COX, ARTHUR N. (T-6)

BECKER, STEPHEN A. (X-2)

DESPAIN, KEITH HOWARD ( $(2-2)$

48-101

SEARCH FOR YOUNG, LUMINOUS OPTICAL

PULSARS IN EXTRAGALACTIC SUPERNOVA

REMNANTS.

ASTROPHYS.J.

V.279, P.157-61. 1984.

MIDOLEDITCH, JOHN (ESS-9)

KRISTIAN, JEROME

48-102

SIMULATION OF STATIONARY AND TRANSIENT GEOPOTENTIAL - HEIGHT EDDIES IN JANUARY AND JULY WITH A SPECTRAL GENERAL

CIRCULATION MODEL.

J. ATMOS. SCl.

V.41, P.1394-1418. 1984

MALONE, ROBERT C. (CTR-6)

PITCHER, ERIC J.

BLACKMON, MAURICEL

PURI, KAMAL

BOURKE, WILLIAM

48-103

SOLAR FLARES - AN EXTREMUM OF

RECONNECTION.

MAGNETIC RECONNECTION IN SPACE AND

LABORATORY PLASMAS, 1983, LOS ALAMOS.

PROC., P.193-201. AGU, 1984.

(538.766/M196RE/1983)

ALSO PUBLISHED IN:

กC,

1983. 11P. MN (LA-UR-83-3704)

COLGATE, STIRLING A. (T-6)
48-104

SOLITON COLLAPSE DURING IONOSPHERIC

HEATING.

T1C.

1984. 5P. MN (LA-UR-84-1067)

SHEERIN, J.P.

NICHOLSON, DWIGHT R.

PAYNE, GERALD L.

DUNCAN, LEWIS M. (ESS-7)

48-105

SOLITON STRUCTURE IN CRYSTALLINE

ACETANILIDE.

PHYS. REV.,

V.30B, P.4703-12. 1984

EILBECK, J. CHRIS (CNLS)

LOMDAHL, PETER S. (CNLS)

SCOTT, ALWYN C. (CNLS)

48-106

SOME INVESTIGATIONS ABOUT THE CARSON OPACITIES.

OBSERVATIONAL TESTS OF THE STELLAR

EVOLUTION THEORY, A. MAEDER \& A.

RENZINI, ED., P.41-6. D. REIDEL PUB.

CO. 1984. (523.8/Ot4TE/1883)

ALSO PUBLLSHED IN:

TIC.

1983. 6P. MN (LA-UR-83-3216)

COX, ARTHUR N. (T-6)

KIDMAN, RUSSELL B. (T-6)

48-107

SOME OBSERVABLE INDICATORS OF

S - PROCESS ENVIRONMENTS.

TiC.

1983. 4P. MN (LA-UR-83-2930)

DESPAIN, KEITH HOWARD (X-2)

48-108

STABILITY OF THELOW DEGREE FIVE MINUTE

SOLAR OSCILLATIONS.

TIC.

1983. 10P. MN (LA-UR-83-3217)

KIDMAN, RUSSELL B. (T-6)

COX, ARTHUR N. $(T-6)$

48-109

STRUCTURE OF THE MAGNETOTAIL AT 220 R E

AND ITS REPONSE TO GEOMAGNETIC ACTIVITY.

GEOPHYS. RES. LETT.

V.11, P.5-7. 1984

HONES, EDWARD W., JR. (ESS-8)

BAKER, DANIEL N. (ESS-8)

BAME, SAMUEL J., JR. (ESS-8)

FELDMAN, WILLAM C. (ESS-8)

GOSLING, JOHNT. (ESS-8)

MC COMAS, DAVID J. (ESS-8)

ZWICKL, RONALD D. (ESS-8)

SLAVIN, J.A

SMITH, EJ.

TSURUTANI, B.T.
48-110

SUPERNOVA ENVELOPE SHOCK ORIGIN OF COSMIC RAYS - A REVIEW.

ADV. SPACE RES.

V.4, P.367-9. 1984.

COLGATE, STIRLING A. (T-6)

48-111

SUPRATHERMAL IONS UPSTREAM FROM

INTERPLANETARY SHOCKS.

J. GEOPHYS. RES.,

V.89A,P.5409-18. 1984.

GOSLING, JOHN T. (ESS-8)

BAME, SAMUEL J., JR. (ESS-8)

FELDMAN, WILLIAM C. (ESS-8)

PASCHMANN, GOTZ

SCKOPKE, $N$.

RUSSEL C.T.

48-112

SYNTHESIS OF COMPLEX MOLECULES IN DENSE INTERSTELLAR CLOUDS VIA GAS-PHASE

CHEMISTRY - A PSEUDO TIME-DEPENDENT

CALCULATION.

ASTROPHYS. J. SUPPL. SER.,

V.56,P.231-56. 1984.

LEUNG, CHUN MING

HERBST, ERIC

HUEBNEA, WALTER F. (T-4)

48-113

TEN YEARS OF VELAX - RAY

OBSERVATIONS

HIGH ENERGY TRANSIENTS IN

ASTROPHYSICS, 1983, SANTA CRUZ.

PROC., P.76-9. AIP, 1984.

(523.015/H638EN/1983)

ALSO PUBLISHED IN:

TrC,

1983. 5P. MN (LA-UR-83-2777)

TERRELL, NELSON JAMES (ESS-9)

PRIEDHORSKY, WILLIAM C. (ESS-9)

48-114

THEORETICAL CALCULATION OF RAPID

$X$ - RAY TRANSIENTS AND RADIUS

EXPANSION.

HIGH ENERGY TRANSIENTS IN

ASTROPHYSICS, 1983, SANTA CRUZ.

PROC., P.298-301. AIP. 1984.

(523.015/H638EN/1983)

ALSO PUBLISHED IN:

TIC,

1983. 4P. MN (LA-UR-83-2744)

STARRFIELD, $\mathrm{S}$.

SPARKS, WARREN M. $(X-5)$

TRURAN, JAMES W.

KENYON, $\mathbf{8}$.

48-115

THREE - DIMENSIONAL COMPUTER MODELNG

OF DYNAMIC RECONNECTION IN THE

MAGNETOTAIL - PLASMOID SIGNATURES IN

THE NEAR AND DISTANT TAIL.

MAGNETIC RECONNECTION IN SPACE AND

LABORATORY PLASMAS, 1983, LOS ALAMOS.

PROC., P264-70. AGU, 1984.

(538.766/M196RE/1983)

ALSO PUBLISHED IN:

ก1C,

1983. 42P. MN (LA-UR-84-0729)

BIRN, J. 
48-116

TWO- AND THREE-DIMENSIONAL NUMERICAL SIMULATIONS OF THE HYDRODYNAMIC PHASE OF THE CORE HELIUM FLASH.

ASTROPHYS.J.,

V.287, P.268-81. 1984.

DEUPREE, ROBERT G. (ESS-5)

48-117

TWO-AND THREE-DIMENSIONAL NUMERICAL SIMULATIONS OF THE CORE HELIUM FLASH.

ASTROPHYS. J.,

V.282, P.274-86. 1984.

DEUPREE, ROBERT G. (ESS-5)

48-118

WAVE-PARTICLE TRANSPORT FROM DENSITY DRIFT INSTABILITIES, A COMPARISON OF LOCAL AND NONLOCAL THEORIES.

J. GEOPHYS. RES.,

V.89A, P.2936-44. 1984.

BERNHARDT, PAUL A. (ESS-7)

48-119

$X$-RAY EMISSION FROM CENTAURUS A. $X$-RAY AND UV EMISSION FROM ACTIVE GALACTIC NUCLEI, 1984, GARCHING. PROC., P.102-4. MPI, 1984.

TERRELL; NELSON JAMES (ESS-9)
48-120

$X$-RAY OBSERVATIONS OF A LARGE SAMPLE OF CATACLYSMIC VARIABLE STARS USING THE EINSTEIN OBSERVATORY.

MON. NOT. R. ASTRON. SOC.,

V.206, P.879-97. 1984.

CORDOVA, FRANCE ANNE (ESS-9)

MASON, K. O.

48-121

X-RAY TEST OF MULTILAYER COATED OPTICS.

STANFORD SYNCHROTRON RADIATION LAB

ACTIVITY REPORT FOR APRIL 1, 1983

DECEMBER 31, 1983. K. CANTWELL, ED.,

P.IX-81. SSRL, 1983. (SSRL-84/01)

GOLUB, L.

SPILLER, EBERHARD

BARTLETT, ROGER J. (P-14)

KANIA, DON R. (P-14)

TRELA, WALTER J. (P-DO)

48-122

1973 X - RAY TRANSIENT VO332+53

ASTROPHYS. J.,

V.285, P.L15-8. 1984.

TERRELL, NELSON JAMES (ESS-9)

PRIEDHORSKY, WILLIAM C. (ESS-9)
48-123

3 - 10 KEV AND 0.1 TO 2 MEV

OBSERVATIONS OF FOUR GAMMA - RAY BURSTS. HIGH ENERGY TRANSIENTS IN

ASTROPHYSICS, 1983, SANTA CRUZ.

PROC., P.378-89. AIP, 1984.

(523.015/H638EN/1983)

ALSO PUBLISHED IN:

TIC,

1983. 13P. MN (LA-UR-83-2657)

LAROS, JOHN G. (ESS-9)

EVANS, W. DOYLE (ESS-9)

FENIMORE, EDWARD E. (ESS-9)

KLEBESADEL, RAY W. (ESS-9)

SHULMAN, $S$.

FRITZ, G.

48-124

3 KEV TO 2 MEV OBSERVATIONS OF FOUR GAMMA - RAY BURSTS.

ASTROPHYS. J.

V.286, P.681-90. 1984.

LAROS, JOHN G. (ESS-9)

EVANS, W. DOYLE (ESS-9)

FENIMORE, EDWARD E. (ESS-9)

KLEBESADEL, RAY W. (ESS-9)

SHULMAN, $S$.

FRITZ, G. 
49-1

"INDEPENDENT-PAIR" PROPERTY OF CONDENSED COHERENT FERMION PAIRS AND DERIVATION OF THE IBM QUADRUPOLE OPERATOR.

PHYS. LETT.

V.138B, P.1-5. 1984.

OTSUKA, TAKAHARU (T-5)

49-2

ANALYSIS OF ANELASTIC RELAXATIONS CONTROLLED BY A SPECTRUM OF RELAXATION TIMES.

TIC,

1983. 26P. MN (LA-UR-84-0138)

COST, JAMES RICHARD (MST-5)

49-3

APPARATUS AND METHOD FOR QUANTITATIVE MEASUREMENT OF SMALL DIFFERENCES IN OPTICAL ABSORPTIVITY BETWEEN TWO SAMPLES USING DIFFERENTIAL INTERFEROMETRY AND THE THERMOOPTIC EFFECT.

PATENTED

MAY 8, 1984, FILED 1982.

(U.S.PATENT-4,447,153)

CREMERS, DAVID A. (CHM-4)

KELLER, RICHARD A. (CHM-2)

49-4

APPARATUS TO DETERMINE THE HEAT CAPACITY AND THERMAL CONDUCTIVITY OF A MATERIAL FROM 1 TO $300 \mathrm{~K}$ IN MAGNETIC FIELDS UP TOQT.

ADVANCES IN CRYOGENIC ENGINEERING, 1983, COLORADO SPRINGS.

PROC., P.425-32. PLENUM, 1984.

ALSO PUBLSHED IN

TiC.

1983. 9P. MN (LA-UR-63-2378)

BARCLAY, JOHN A. (P-10)

STEWART, WALTER F. (P-10)

OVERTON, WILLIAM C., JR. (P-10)

CHESEBOROUGH, ROBERT (P-10)

MC CRAY, MARK S. (P-10)

MC MILLAN, DEAN E. (P-9)

49-5

BREATHERS AND PHOTOINDUCED ABSORPTION

IN POLYACETYLENE.

PHYS. REV. LETT.

V.52, P.671-4. 1984.

BISHOP, ALAN R. (T-11)

CAMPBELL, DAVID K. (CNLS)

LOMDAHL, PETER S. (CNLS)

HOROVITZ, BARUCH

PHILLPOT, SIMON R
49-6

CALCULATION OF SHOCK PROBLEMS BY USING FOUR DIFFERENT SCHEMES.

TIC,

1984. 12P. MN (LA-UR-84-0344)

LEE, WEN HO $(X-7)$

WHALEN, PAUL P. (X-DO)

49-7

CALCULATION OF STRUCTURES AND MAGNETIC ORIENTATIONS IN SOLID ALPHA AND BETA - OXYGEN 2 AS A FUNCTION OF PRESSURE.

TIC,

1983. 7P. MN (LA-UR-83-2241)

ETTERS, RICHARD D. (M-6)

HELMY, A.

KOBASHI, $\mathrm{K}$.

49-8

CAPABILITIES OF BROADLY - TUNABLE

FREE - ELECTRON LASERS.

NEW LASERS FOR ANALYTICAL AND

INDUSTRIAL CHEMISTRY, 1984,

LOS ANGELES. PROC., P.90-5. SPIE

1984.

ALSO PUBLISHED IN:

TIC,

1984. 19P. MN (LA-UR-84-1104)

NEWNAM, BRIAN E. (CHM-6)

49-9

CIRCULAR FREE-ELECTRON LASER.

PATENTED

APR. 10, 1984, FILED 1982.

(U.S.PATENT-4,442,522)

BRAU, CHARLES A. (AT-DO)

KURNIT, NORMAN A. (CHM-6)

COOPER, RICHARD K. (AT-6)

49-10

CLASSICAL AND QUANTUM HARD SPHERE

FLUIDS - THEORY AND EXPERIMENT.

ANN. PHYS.

V.153, P.283-98. 1983.

BAKER, GEORGE A., JR. (T-11)

GUTIERREZ, G.

DE LLANO, $M$

49-11

COHERENT DENSITY GRADIENTS IN WATER

COMPRESSED BY A MODULATED SHOCK WAVE.

PHYS. FLUIDS,

V.27, P.2390-3. 1984.

BENJAMIN, ROBERT F. (M-6)

TREASE, HAROLD (X-7)

SHANER, J. W. (M-6)
49-12

COLLECTIVE, STOCHASTIC AND

NONEQUILIBRIUM BEHAVIOR OF

HIGHLY EXCITED HADRONIC MATTERS.

NUCL. PHYS.,

V.A418, P.501C-23C. 1984.

ALSO PUBLISHED IN:

TIC,

1983. 24P. MN (LA-UR-83-3067)

CARRUTHERS, PETER A. (T-DO)

49-13

COMPILATION OF INTERIM TECHNICAL

RESEARCH MEMORANDA - I.

LOS ALAMOS,

1984. 35P. (LA-10084-MS, VOL. 1)

SHANAHAN, WILLIAMR. $(X-\theta)$

49-14

COMPRESSION OF SOLID NITROGEN AT

296 K FROM 5 TO 10 GPA.

J. CHEM. PHYS.

V.80, P.1309-11. 1984.

OLINGER, BARTON W. (M-6)

49-15

COMPTON SCATTERING OF PHOTONS FROM

ELECTRONS IN THERMAL (MAXWELLIAN)

MOTION - ELECTRON HEATING.

LOS ALAMOS

1984. 45P. (LA-10065-MS)

DEVANEY, JOSEPH J. $(X-6)$

49-16

COMPUTERIZED STUDY OF SEVERAL

ELECTROSTATIC, SURFACE - IONIZATION

ION SOURCE CONFIGURATIONS.

LOS ALAMOS,

1984. 27P. (LA-10107)

BALESTRINI, SILVIO J. (CNC-11)

SCHUSTER, BURTON G. (H-5)

49-17

CRITICAL DYNAMICS NEAR THE OSCILLATORY

INSTABILTYY IN RAYLEIGH - BENARD

CONVECTION.

PHYS. REV. LETT.,

V.53, P.1567-70. 1984.

ECKE, R. (P-10)

MAENO, YOSHITERU

HAUCKE, HANS

WHEATLEY, JOHN C. (P-10) 
49-18

CRYSTAL BOX - RARE DECAYS OF THE MUON.

LOW ENERGY TESTS OF CONSERVATION

LAWS IN PARTICLE PHYSICS, 1983,

BLACKSBURG. PROC., P.242-53. AIP

1984. (539.72/L912EN/1983)

BOLTON, RICHARD D. (MP-4)

CARLNI, ROGER D. (MP-4)

BOWMAN, J. DAVID (MP-4)

COOPER, MARTIN D. (MP-4)

HALLIN, AKSEL (MP-4)

FRANK, JAMES S. (MP-4)

HOFFMAN, CYRUS M. (MP-4)

MATIS, H.S. (MP-4)

MISCHKE, RICHARD E. (MP-4)

NAGLE, DARRAGH E. (MP-DO)

MARIAM, FRANK (MP-13)

SANDBERG, V. D. (MP-4)

SENNHAUSER, URS (MP-4)

SANDERS, GARY H (MP-13)

WERBECK, RICHARD D. (MP-7)

WLUIAMS, ROBERT

HOFSTADTER, R

HUGHES, E. BARRIE

WILSON, JOHN

ROLFE, J. R.

WRIGHT, S. C.

GROSNICK, D.

HOGAN, GARY E. (MP-13)

HIGHLAND, V. L

VAN, $M$.

49-19

DELTA. THE FIRST PION NUCLEON RESONANCE ITS DISCOVERY AND APPLICATIONS.

LOS ALAMOS,

1984. 10P. (LALP-84-027)

NAGLE, DARRAGH E. (MP-DO)

49-20

DENSE MOLECULAR THEPMODYNAMICS.

TIC.

1983. 5P. MN (LA-UR-83-2232)

JOHNSON, JAMES D. (T-4)

SHAW, MILTON S. (T-14)

HOLIAN, BRAD LEE $(T-4)$

49-21

DETECTION OF FREE SURFACE MOTION USING

A MASKED APERTURE TECHNIQUE.

REV. SCI. INSTRUM.

V.55, P.1301-3. 1984

WINLER, MAX A. (M-5)

HIXSON, ROBERT S. (X-5)

SHANER, J. W. (M-6)

49-22

DEVICE AND METHOD FOR SEPARATING

OXYGEN ISOTOPES.

PATENTED

MAR. 20, 1984, FILED 1978.

(U.S.PATENT-4,437,958)

ROCKWOOD, STEPHEN D. (NSP/IF)

SANDER, ROBERT K. (CHM-4)
49-23

DRAG OF EVAPORATING OR CONDENSING

DROPLETS IN LOW REYNOLDS NUMBER FLOW.

PHYS. FLUIDS.

V.27, P.1351-7. 1984.

DUKOWICZ, JOHN K. (T-3)

49-24

EDXD STUDIES AT HIGH PRESSURES AND

CRYOGENIC TEMPERATURES USING SYNCHROTRON

RADIATION - APPLICATION TO THE TWO

CRITICAL POINTS IN BETA - ALPHA PHASE

BOUNDARY OF CERIUM ALLOYS.

STANFORD SYNCHROTRON RADIATION LAB.

ACTIVIYY REPORT FOR APRIL 1, 1982 .

MARCH 31, 1983.

P.VII-32-3. SSRL, 1983. (SSRL-83/01)

SKELTON, E.F.

WEBB, A. W.

ELAM, WEST T.

WOLF, S. A

QADRI, SYED B

HUANG, CHAO YUAN (P-10)

CHAIKIN, PAUL $M$.

LACOE, RONALD C.

GSCHNEIDNER, KARL A.

49-25

EFFECTIVE SPHERICAL POTENTIALS FOR

DETONATION PRODUCTS EOS.

TIC,

1983. 12P. MN (LA-UR-63-2541)

SHAW, MILTON S. (T-14)

HOLIAN, BRAD LEE (T-4)

JOHNSON, JAMES D. (T-4)

49-26

EFFECTS OF FOCUSING ON HRS FOCAL - PLANE

POLARIMETER MEASUREMENTS.

LOS ALAMOS

1984. 25P. (LA-10153-MS)

FERGERSON, RAYMOND W. (MP-10)

49-27

EFFECTS OF MICROSTRUCTURE ON THE SPEED

AND ATTENUATION OF ELASTIC WAVES - SOME

RESULTS FOR POROUS MATERIALS.

REVIEW OF PROGRESS IN QUANTITATIVE

NONDESTRUCTIVE EVALUATION, 1983

BOULDER. PROC. P.1129-35. PLENUM,

1984.

GUBERNATIS, JAMES E. (T-11)

DOMANY, EYTAN

49-28

EFFECTS OF MICROSTRUCTURE ON THE SPEED

AND ATTENUATION OF ELASTIC WAVES IN

POROUS MATERIALS.

WAVE MOTION,

V.6, P.579-89. 1984.

GUBERNATIS, JAMES E. (T-11)

DOMANY, EYTAN
$49-29$

EFFECTS OF RADIAL COUNTERFLOW ON

ROTATING HELUUM II.

JUBILEE CONF. ON HELIUM - 4, 75TH

1983, ST. ANDREWS. PROC., P.62-3.

WORLD SCIENTIFIC, 1983

(536.56/J91CO/1983)

CAMPBELL, LAURENCE J. (T-11)

49-30

EFFECTS OF THE INTERNALLY PRODUCED

NONTHERMAL ELECTRONS ON THE TEMPERATURE

DIAGNOSTICS OF A HOLLOW GAS SHELL

Z-PINCH.

APPL. PHYS. LETT.

V.44, P.667-9. 1984.

HAMMEL, BRUCE A. (P-1)

JONES, LARRY A. (P-6)

49-31

EFFICIENT STIMULATED RAMAN SCATTERING

DUE TO ABSENCE OF SECOND STOKES GROWTH.

OPT. LETT.

V.9. P.353-5. 1984

CARLSTEN, JOHN L. (CHM-6)

TELIE, JOHN M. (CHM-6)

WENZEL, ROBERT G. (CHM-6)

$49-32$

ELASTIC WAVE SCATTERING BY A

SURFACE - BREAKING OR SUBSURFACE

PLANAR CRACK - II. THREE-DIMENSIONAL

GEOMETRY.

J.APPL. PHYS.

V.56, P.713-24. 1984.

VSSCHER, WILLAM MAURITS (T-11)

$49-33$

ELECTRICAL CONDUCTIVITY OF AN

ARBITRARILY DENSE PLASMA.

LOS ALAMOS

1984. 79P. (LA-09872-MS)

RINKER, GEORGE A., JR. (T-4)

$49-34$

ELECTRODYNAMIC INTERPRETATION OF VRTUAL CATHODE OSCILLATIONS.

LOS ALAMOS

1984. 12P. (LA-09908)

KADISH, ABRAHAM $(\alpha-8)$

49-35

ELECTRON - ELECTRON INTERACTION EFFECTS

ON PEIERLS DIMERIZATION IN A HALF

FILLED BAND.

PHYS. REV,

V.29B, P.1824-38. 1984.

DIXT, S. N.

MAZUMDAR, SUMIT (CNLS) 
$49-36$

ELECTRONIC EXCITATION OF ATOMS AND MOLECULES BY ELECTRON IMPACT IN A UNEAR ALGEBRAIC, SEPARABLE POTENTIAL APPROACH.

J. PHYS. $B$,

V.17, P.L235-9. 1984

COLLINS, LEE A. (T-4)

SCHNEIDER, BARRY I. (T-12)

49-37

ENERGETIC PLASMA BEAM PROPAGATION IN WEAK AND STRONG MAGNETIC FIELDS.

TIC,

1983. 5P. MN (LA-UR-83-2580)

SHANAHAN, WILLIAM R. $(X-8)$

49-38

ENERGY DEPOSITION FROM PARTICLE BEAMS.

LOS ALAMOS,

1984. 27P. (LA-09683-MS)

OSBORNE, $W . z$.

49-39

EQUATION OF STATE FOR THE DETONATION

PRODUCTS OF SEVERAL SIMPLE EXPLOSIVES.

TIC,

1983. 14P. MN (LA-UR-83-1540)

SHAW, MILTON S. $(\mathrm{T}-14)$

HOLIAN, BRAD LEE $(T-4)$

JOHNSON, JAMES D. (T-4)

$49-40$

EQUATION OF STATE FOR TITANIUM

DIHYDRIDE.

LOS ALAMOS,

1984. 15P. (LA-09895-MS)

BENNETT, BARD I., JR. $\left(T^{-4}\right)$

49-41

EQUATION OF STATE OF

POLYTETRAFLUOROETHYLENE TO

BO GPA.

J. CHEM. PHYS.,

V.80, P.5203-17. 1984.

MORRIS, CHARLES E. (M-6)

FRITZ, JOSEPH N. (M-6)

MC QUEEN, ROBERT G. (M-6)

$49-42$

EQUATION OF STATE OF FLUID AMMONIA FROM P-V-T AND ULTRASOUND MEASUREMENTS TO 12 KBAR.

TIC,

1983. 9P. MN (LA-UR-83-2180)

MILLS, ROBERT L. (P-10)

LEBENBERG, DONALD H. (P-10)

PRUZAN, PHILIPPE

$49-43$

EQUATION OF STATE OF SODIUM

J. CHEM. PHYS.

V.80, P.2864-70. 1984.

FRITZ, JOSEPH N. (M-6)

OLINGER, BARTON W. (M-6)
$49-44$

EVOLUTION OF LONG PULSES IN A TAPERED

WIGGLER FREE ELECTRON LASER.

LOS ALAMOS CONFERENCE ON OPTICS,

1983, LOS ALAMOS AND SANTA FE.

PROC., P.131-8. SPIE, 1983.

(535/L8790P/1983)

ALSO PUBLISHED IN:

Tic,

1983. 10P. MN (LA-UR-83-2411)

GOLDSTEIN, JOHN C. (X-1)

49-45

EXCITATION OF MOLECULES BY ELECTRON

IMPACT.

ELECTRON-MOLECULE INTERACTIONS AND

THEIR APPLICATIONS, LG.

CHRISTOPHOROU, ED., P.155-249.

ACADEMIC PRESS, 1984.

(539.721/E38MON.1)

TRAWMAR, $\mathbf{S}$.

CARTWRIGHT, DAVID C. (OPR/FRA)

49-46

EXPERIMENT TO DETERMINE PROPERTIES

OF PACKED PARTICLE BEDS AND

REGENERATORS AT CRYOGENIC TEMPERATURES.

nc,

1983. 9P. MN (LA-UR-83-2351)

ALSO PUBLISHED IN:

ADV. CRYOG. ENG.,

V.29. P.605-12. 1984.

BARCLAY, JOHNA. (P-10)

OVERTON, WILIAM C., JR. (P-10)

STEWART, WALTER F. (P-10)

SARANGI, SUNIL (P-10)

$49-47$

EXTRACTION OF H MINUS BEAMS FROM A

MAGNETICALLY FILTERED MULTICUSP

SOURCE.

LOS ALAMOS.

1984. 7P. (LA-09931)

YORK, ROBBIE LYNN (MP-11)

STEVENS, RALPH R., JR. (MP-DO)

LEUNG, K. N.

EHLERS, K.W.

49-48

EXTRACTION OF HYDROGEN MINUS BEAMS FROM

A MAGNETICALLY FILTERED MULTICUSP

SOURCE.

REV. SCI. INSTRUM.,

V.55, P.681-6. 1984.

YOAK, ROBBIE LYNN (MP-11)

STEVENS, RALPH R., JR. (MP-DO)

LEUNG, K. N.

EHLERS, K. W:

$49-49$

FILAMENTATION INSTABILTY OF ION ACOUSTIC WAVES DRIVEN BY A SCATTERED

RELATIVISTIC BEAM.

TIC.

1983. 5P. MN (LA-UR-83-2671)

LEE, HUAN $(\alpha-8)$

JONES, MICHAEL E. (X-8)
49-50

FIRST OPERATION OF THE LOS ALAMOS

FREE - ELECTRON LASER OSCILLATOR.

INTERNATIONAL CONFERENCE ON LASERS,

1983, SAN FRANCISCO. PROC., P.313-20.

STS PRESS, 1983.

ALSO PUBLISHED IN:

กC.

1983. 8P. MN (LA-UR-84-0202)

WARREN, ROGER W. (AT-7)

NEWNAM, BRIAN E. (CHM-6)

STEIN, WILLIAME. (AT-7)

WINSTON, JOHN G (E-7)

SHEFFIELD, RICHARDL (AT-7)

LYNCH, MICHAEL T. (WX-7)

COLDSTEIN, JOHN C. (X-1)

WHITEHEAD, MICHAELC. (CHM-6)

NORRIS, O. ROY (AT-7)

LUEDEMANN, GARY (AT-5)

GIBSON, TED O. (AT-7)

HUMPHAY, CHRIS M. (AT-7)

49-51

FORCE BALANCE LIMITING CURRENT FOR

CHARGED PARTICLE BEAMS.

LOS ALAMOS,

1984. 6P. (UA-10150)

KADISH, ABRAHAM (X-8)

49-52

FORMATION OF VIRTUAL CATHODES AND MICROWAVE GENERATION IN RELATIVISTIC

ELECTRON BEAMS.

PHYS. FLUIDS,

V.27, P.1570-2. 1984.

KWAN, THOMAS J. T. $(X-8)$

THODE, LESTER E. $(X-8)$

49-53

FOURIER-TRANSFORM RING LASER.

J. OPT. SOC. AM.,

V.1A, P.537-40. 1984.

SKLAR, EDWARD (CHM-6)

$49-54$

FREE - ELECTRON LASERS AND RELATED

TOPICS.

UNEAR ACCELERATOR CONFERENCE

1984, SEEHEIM, PROC., P.256-9.

1984. (GSI-84-11)

WATSON, J.M. (AT-4)

$49-55$

FRICTIONAL HOT-SPOTS AND PROPELLANT

SENSITIVITY.

MATERIALS RESEARCH SOCIETY SYMPOSIA,

1983, BOSTON. PROC., P.373-80.

ELSEVIER, 1984.

ALSO PUBLISHED IN:

TIC,

1983. 10P. MN (LA-UR-83-3278)

DIENES, JOHN K. (T-3) 
49-56

GASDYNAMIC FOCUSING FOR SAMPLE CONCENTRATION IN ULTRASENSITIVE

ANALYSIS.

APPL. OPT.

V.23,P.2146-51. 1984.

KELLER, RICHARD A. (CHM-2)

NOGAR, NICHOLAS S. (CHM-2)

49-57

GENERALIZATION OF THE CHILD-LANGMUIR

RELATION FOR ONE-DIMENSIONAL

TIME-DEPENDENT DIODES.

LOS ALAMOS

1984. 9P. (LA-10207-MS)

KADISH, ABRAHAM $(X-8)$

PETER, WILLIAM K. $(X-8)$

JONES, MICHAEL E. (X-8)

49-58

GENERALIZED POISSON BRACKETS AND

NONLINEAR LIAPUNOV STABILITY.

APPLICATION TO REDUCED MHD.

TIC,

1984. 7P. MN (LA-UR-84-0942)

HAZELTINE, R. D.

HOLM, DARRYL D. (CNLS)

MARSDEN, JERROLD E. (CNLS)

MORRISON, P. J.

49-59

HIGH - ENERGY OPTICALLY PUMPED IODINE

LASER - I - KINETICS IN AN OPTICALLY

THICK MEDIUM.

J. APPL. PHYS.

V.55, P.2659-70. 1984.

COHEN, JAMES S. (T-12)

JUDD, O'DEAN P. (NSP/DST)

49-60

HIGH - POWER COHERENT MICROWAVE

GENERATION FROM OSCILLATING VIRTUAL

CATHODES.

PHYS. FLUIDS,

V.27, P.228-32. 1984

KWAN, THOMAS J. T. $(\mathrm{X}-8)$

49-61

HIGH - TEMPERATURE SERIES EXPANSION

OF DELTA 4 CHI/DELTA H 4 FOR A

GENERAL CONTINUOUS - SPIN ISING

MODEL.

LOS ALAMOS,

1984. 58P. (LA-10275-MS)

JOHNSON, JAMES D. (T-4)

BAKER, GEORGE A., JR. (T-11)

49-62

IMAGERY OF PHYSICS IN PSYCHOLOGY

A PHYSICIST'S REACTIONS.

TIC.

1983. 28P. MN (LA-UR-84-0073)

SANDERS, GARY H (MP-13)
49-63

INDUCTIVELY STABILIZED EXCIMER LASERS.

TIC.

1984. TP. MN (LA-UR-84-1190)

SZE, ROBERT C. (CHM-5)

49-64

INORGANIC FLUORESCENT SCREEN

PROPERTIES IMPORTANT FOR MEV

RADIATION IMAGERY.

LOS ALAMOS,

1984. 17P. (LA-10290-MS)

BERZINS, GEORGE J. (ESS-9)

LUMPKIN, ALEX H. (P-DO)

SMITH, HARRY L.

49-65

INSTABILTYY OF THE BOUNDARY LAYER

BETWEEN A STREAMING PLASMA AND A

VACUUM MAGNETIC FIELD.

PHYS. FLUIDS

V.26, P.2276-80. 1984.

PETER, WILLIAM

RON, AMIRAM

ROSTOKER, NORMAN

49-66

INTELLIGENT OPTICAL DESIGN PROGAAM.

APPLICATIONS OF ARTIFICIAL

INTELLIGENCE, 1984, ARLINGTON.

PROC., P.104-12. SPIE, 1984.

(001.535/A652AR/1984)

ALSO PUBLISHED IN

TIC,

1984. 15P. MN (LA-UR-84-0910)

BOHACHEVSKY, IHOR ORESTES WAC

VISWANATHAN, VRIDDHACHALAM K. (P-5)

WOODFIN, GREG L. (P-5)

49-67

INTERPLAY BETWEEN SUPERCONDUCTIVITY AND

ITINERANT FERROMAGNETISM IN

(VTTRIUM) 9 (COBALT) 7 AT HIGH PRESSURE.

MATERIALS RESEARCH SOCIETY SYMPOSIA,

1984, ALBANY. V.22, P.57-64. 1984.

ALSO PUBLISHED IN

TIC,

1983. 11P. MN (LA-UR-83-2830)

HUANG, CHAO YUAN (P-10)

WOLF, S. A.

OLSEN, CLAYTONE. (MST-13)

FULIER, WENDY WEBB

HUANG, JUI HAN

TING, C. $\mathbf{S}$.

49-68

LANDSLIDE MODEL. FOR THE 1975 HAWAII

TSUNAMI.

SCI. TSUNAMI HAZARDS,

V.2, P.71-8. 1984

MADER, CHARLES L. (T-14)
49-69

LEON RESPONDS (AUG 13, 1984 ISSUE

OF PHYSICAL REVIEW LETTERS)

PHYS. REV. LETT.

V.53, P.739. 1984.

LEON, MELVIN (MP-3)

49-70

LIMITATIONS ON UNIVERSALTTY IN THE

CONTINUOUS - SPIN ISING MODEL

J. PHYS. A,

V.17, P.L275-8. 1984

BAKER, GEORGE A., JR. (T-11)

JOHNSON, JAMES D. (T-4)

49-71

LOCALIZATION IN F - SHELL METALS.

PHYS. REV.,

V.29B, P.2917-25. 1984.

HARRISON, WALTER A

49-72

LOS ALAMOS FREE - ELECTRON LASER

OSCILLATOR EXPERIMENT - PLANS AND PRESENT STATUS.

FREE-ELECTRON GENERATORS OF

COHERENT RADIATION, 1983,

ORCAS ISLAND, WASHINGTON. PROC.,

P. 130-6. SPIE, 1984.

(621.366/F853EL/1983)

ALSO PUBLISHED IN:

TIC.

1983. 8P. MN (LA-UR-83-2443)

WARREN, ROGER W. (AT-7)

FRASER, JOHN S. (AT-7)

STEIN, WILLIAM E. (AT-7)

WINSTON, JOHN G (E-7)

SWANN, THOMAS A. (P-5)

LUMPKIN, ALEX H. (P-DO)

SHEFFIELD, RICHARD L. (AT-7)

SOULD, JON E. (P-5)

NEWNAM, BRIAN E. (CHM-6)

BRAU, CHARLES A. (AT-DO)

WATSON, J. M. (AT-4)

49-73

LOW HYSTERESIS MATERIALS FOR MAGNETIC REFRIGERATION - GADOLINIUM 1-X

ERBIUM X ALUMINUM 2

J. APPL. PHYS.,

V.55, P.2609-10. 1984.

ALSO PUBLISHED IN:

TIC.

1983. 10. MN (LA-UR-83-2890)

ZIMM, C. B. (P-10)

BARCLAY, JOHN A. (P-10)

JOHANSON, WILLIAM A. (MST-5)

49-74

LOW-TEMPERATURE PRESSURE VARIATIONS IN

A SELF - CLAMPING PRESSURE CELL.

REV. SCI. INSTRUM.,

V.55, P.231-4. 1984.

THOMPSON, JOE D. (P-10) 
\$9-75

LYAPUNOV STABILITY CONDITIONS FOR A RELATIVISTIC MULTIFLUID PLASMA.

TIC,

1984. 9P. MN (LA-UR-84-1127)

HOLM, DARRYL D. (CNLS)

KUPERSHMIDT, BORIS ABRAM (CNLS)

49-76

MAXIMUM SUPERCOOLING IN LIQUID 3 HELIUM MINUS 4 HELIUM MIXTURES NEAR THE TRICRITICAL POINT.

TIC,

1984. 3P. MN (LA-UR-84-0904)

SINHA, DIPEN N. (P-10)

HOFFER, JAMES K. (P-10)

49-77

MEASURED HUGONIOT STATES OF A TWO-

ELEMENT FLUID, OXYGEN PLUS NITROGEN,

NEAR TWO MEGAGRAMS PER CUBIC METER SHOCK WAVES IN CONDENSED MATTER, 1983, SANTA FE. PROC., P.49-52.

ELSEVIER, 1984.

SCHOTT, GARRY L. (M-1)

49-78

MEASURED HUGONIOT STATES OF A TWOELEMENT FLUID, OXYGEN 2 PLUS NITROGEN 2 NEAR 2 MG/M CUBED.

TIC,

1983. 5P. MN (LA-UR-83-2172)

SCHOTT, GARAY L. (M-1)

49-79

MEASUREMENT OF THE PP GOING TO PID SPIN CORRELATION PARAMETERS A SL AND A LL AT ENERGIES BETWEEN 500 AND $800 \mathrm{MEV}$.

LOS ALAMOS,

1984. 102P. (LA-10289-T)

BARLOW, D.

49-80

MEASUREMENTS OF THE THERMAL CONDUCTIVITY AND VISCOSITY OF LIQUID PROPYLENE.

J. CHEM. ENG. DATA

V.29,P.56-9. 1984

SWIFT, GREG W. (P-10)

MIGLIORI, ALBERT (P-10)
49-81

MEASURMENT OF DELTA SIGMA SUB - LAND

C SUB - $(L, L ; 0,0)$ IN PROTON-PROTON

SCATTERING BETWEEN 300 AND 800 MEV.

PHYS. REV.

V.29D, P.2435-64. 1984.

AUER, I.P.

DITZLER, W. R.

HILL, DANIEL

IMAI, K.

SPINKA, $H$.

STANEK, R.

TOSHIOKA, K.

UNDERWOOD, D.

WAGNER, $R$.

YOKOSAWA, A.

HOFFMAN, EARL WAYNE (MP-1)

JARMER, JOHN J. (MP-7)

BURLESON, GEORGE R.

COTTINGAME, W. B.

GREENE, S. J.

STUART, $\mathbf{S}$.

49-82

MELTING OF METALS ABOVE 100 GPA.

MATERIALS RESEARCH SOCIETY SYMPOSIA,

1983, ALBANY. V.22, P.137-41. 1984

ALSO PUBLISHED IN:

TIC,

1983. 6P. MN (LA-UR-83-2248)

SHANER, J. W. (M-6)

BROWN, J. MICHAEL

MC QUEEN, ROBERT G. (M-6)

49-83

METHOD OF FORMING METALLIC COATINGS ON POLYMERIC SUBSTRATES.

PATENTED

AUG. 7, 1984, FILED 1983.

(U.S.PATENT-4,464,416)

LIEPINS, RAIMOND (MST-6)

49-84

MICROWAVE GENERATORS - OSCILLATING

VIRTUAL CATHODES AND REFLEXING

ELECTRONS.

TIC,

1983. 4P. MN (LA-UR-B3-2602)

KWAN, THOMAS J. T. $(x-8)$

THODE, LESTER E. $(X-B)$

49-85

MOLECULAR DYNAMICS CALCULATIONS OF THE HARD - SPHERE EQUATION OF STATE.

J. STAT. PHYS.,

V.35, P.321-40. 1984

ERPENBECK, JEROME J. (T-11)

WOOD, WILLIAM W. (T-11)

49-86

MONTE CARLO SIMULATION OF ELECTRON/ PHOTON/CERENKOV CASCADES IN GENERALIZED GEOMETRY.

TRANS. AM. NUCL. SOC.

V.46, P.434-5. 1984.

MACK, JOSEPH M., JR. (X-6)

JORDAN, THOMAS M.
49-87

MU PLUS SR STUDY OF SOME MAGNETIC

SUPERCONDUCTORS.

HYPERFINE INTERACT.

V.17-19, P.509-14. 1984.

HUANG, CHAO YUAN (P-10)

ANSALDO, E.J.

BREWER, JESS HENRY

HARSHMAN, D. R.

CROWE, KENNETH M.

ROSENBLUM, STEPHEN S.

CLAWSON, CARL W.

FISK, ZACHARY (MST-5)

LAMBERT, STEVENE.

TORIKACHVILI, MILTONS.

MAPLE, MERRILL $B$.

49-88

MULTIPLE SCATTERING EFFECTS IN

SPATIAL FREQUENCY FILTERING.

APPL. OPT.

V.23, P.4124-30. 1984. ZARDECKI, ANDREW (T-DO)

GERSTL, S. A.W. (T-DOT)

EMBURY, JANON F.

49-89

MULTIPRESSURE REGULARIZATION FOR

MULTIPHASE FLOW.

PHYS. LETT.

V.106A, P.165-8. 1984

HOLM, DARRYL D. (CNLS)

KUPERSHMIDT, BORIS ABRAM (CNLS)

49-90

MULTISTEP PUMPING SCHEMES FOR SHORT -

WAVE LASERS.

LASER INTERACTION AND RELATED

PLASMA PHENOMENA, H. HORA AND G. H.

MILEY, EDS., P.107-24. PLENUM, 1984.

(535/L343IN/N.6)

BALDWIN, GEORGE C. (P-3)

49-91

NOISE - INDUCED INTERMITTENCY IN A

CONVECTING DILUTE SOLUTION OF HELUUM 3

IN SUPERFLUID HELIUM 4.

PHYS. REV. LETT.

V.53, P.2090-2. 1984

HAUCKE, HANS (P-10)

ECKE, R. (P-10)

MAENO, YOSHITERU

WHEATLEY, JOHN C. (P-10)

49-92

NONLINEAR EVOLUTION OF THE LOWER HYBRID

DRIFT INSTABILITY.

PHYS. FLUIDS,

V.27, P.2682-92. 1984.

BRACKBILL, JEREMIAH U. (X-1)

FORSLUND, DAVID W. $(x-1)$

QUEST, KEVIN B. $(X-1)$

WINSKE, DAN $(X-1)$ 


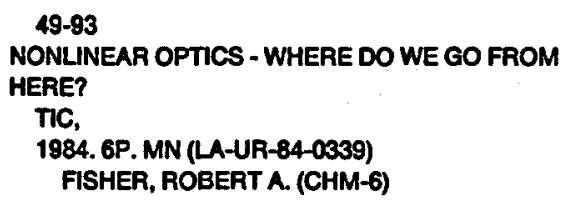

49-94

ON OBTAINING THE ZERO TEMPERATURE EQUATION OF STATE FROM SHOCK DATA. LOS ALAMOS,

1984. 15P. (LA-09890-MS)

BENNETT, BARD I., JR. (T-4)

49-95

ON THE INTERPLAY BETWEEN DIELECTRIC SCREENING AND CORE HOLE RELAXATION IN SOFT X-PAY ABSORPTION.

J. PHYS. B,

V.17, P.L253-7. 1984.

ZANGWILL, ANDREW

UBERMAN, DAVID A. (T-4)

49-96

ONSET OF CHAOS IN DUFFING OSCILLATOR

SYSTEMS.

COHERENCE AND QUANTUM OPTICS, 5TH, 1983, ROCHESTER. PROC., P.729-35.

PLENUM, 1984.

ACKERHALT, JAY R. (T-12)

GALBRAITH, HAROLD W. (T-12)

MILONNI, PETER W. (T-12)

$49-97$

OPTICAL ACTIVITY AND PARITY VIOLATION.

PHYSICS OF OPTICAL RING GYROS, 1984. SNOWBIRD. PROC., P.128-36. SPIE,

1984. (629.135/P5780P/1984)

ELLIOTT, C. JAMES $(\alpha-1)$

SMALL, JAMES $G$.

49-98

OPTICAL EFFECTS OF THE GRAVITATIONALLY INDUCED CONCENTRATION GRADIENT IN

TRICRITICAL 3 HELIUM - 4 HELIUM.

TIC.

1984. 3P. MN (LA-UR-84-0944)

SHIAH, SHOOU-SHINN (P-10)

HOFFER, JAMES K. (P-10)

$49-99$

OPTICAL ENGINEERING AT LOS ALAMOS - A

HISTORY.

LOS ALAMOS CONFERENCE ON OPTICS,

PROC., P.2-16. SPIE, 1983.

(535/L879OP/1983)

ALSO PUBLISHED IN:

TIC.

1984. 16P. MN (LA-UR-83-0953)

BRIXNER, BERLYN (M-5)
49-100

OPTICAL PROPERTIES OF EXPLOSIVE - DRIVEN

SHOCK WAVES IN NOBLE GASES.

LOS ALAMOS CONFERENCE ON OPTICS,

1983, LOS ALAMOS AND SANTA FE.

PROC., P.312-21. SPIE, 1983.

(535/L879OP/1983)

ALSO PUBUSHED IN:

TIC.

1983. 11P. MN (LA-UR-83-1928)

JONES, CLAUDE R. (CHM-6)

DAVIS, WILLIAM C. (M-3)

49-101

OVERVIEW OF RAYLEIGH - TAYLOR

INSTABILTYY

PHYSICA,

V.12D, P.3-18. 1984

ALSO PUBLISHED IN:

TIC.

1983. 47P. MN (LA-UR-83-2130)

SHARP, DAVID H. (T-8)

49-102

OVERVIEW OF RAYLEIGH-TAYLOR INSTABILTY.

INTL CONF. OF THE CENTER FOR

NONLINEAR STUDIES, 3RD, 1983,

LOS ALAMOS. PROC., P.3-18.

NORTH-HOLLAND PHYSICS PUBL., 1984.

SHARP, DAVID H. (T-8)

49-103

P-STATE SUPERCONDUCTIVITY -

A ROUTINE TEST LEADS TO AN

EXTRAORDINARY DISCOVERY.

LOS ALAMOS SCI.

SPRING, NO.10, P.59-67. 1984.

STEWART, GREGORY R. (MST-5)

FISK, ZACHARY (MST-5)

WILLIS, JEFFREY D. (MST-5)

SMITH, JAMES L. (MST-5)

49-104

PASSIVE QUENCH ARREST BY A CHIMNEY

INDUCED DELUGEAT EVERY QUENCH FRONT.

ADV. CRYOG. ENG.,

V.29.P.225-35. 1984.

SYDORIAK, STEPHEN G. (P-10)

49-105

PATTERN FORMATION BY SHOCK PROCESSES.

INTL. CONF. OF THE CENTER FOR

NONUNEAR STUDIES, 3RD, 1983

LOS ALAMOS. PROC., P.154-62.

NORTH-HOLLAND PHYSICS PUBL., 1984

(530.15/161CE/3RD/1983)

ALSO PUBLISHED IN:

TIC,

1983. 22P. MN (LA-UR-83-2113)

SHANER, J.W. (M-6)
49-106

PERFORMANCE OF LAYERED SYNTHETIC MICROSTRUCTURES IN THE SOFT X-RAY ENERGY RANGE.

STANFORD SYNCHROTRON RADIATION LAB.

ACTIVITY REPORT FOR APRIL 1, 1982

MARCH 31, 1983. K. CANTWELL, ED.

P.VI-34. SSRL, 1983. (SSRL-83/01)

BARTLETT, ROGER J. (P-14)

DAY, ROBERT H. (P-14)

KANIA, DON R. (P-14)

TRELA, WALTER J. (P-DO)

KALLNE, ELISABETH (P-1)

BARBEE, TROY $W$.

49-107

PERFORMANCE OF MULTILAYER DISPERSION

ELEMENTS WITH 80 TO $500 \mathrm{EV}$.

SCIENCE WITH SOFT X-RAYS, 1983,

UPTON. PROC., P.23-6. SPIE, 1983.

ALSO PUBLISHED IN

TIC,

1983. 5P. MN (LA-UR-83-2837)

BARTLETT, ROGER J. (P-14)

KANIA, DON R. (P-14)

TRELA, WALTER J. (DIR-O)

KALLNE, ELISABETH (P-1)

LEE, PING (P-4)

SPILLER, EBERHARD

49-108

PERFORMANCE OF TRANSITION METAL - CARBON

MULTILAYER MIRRORS FROM 80 TO $350 \mathrm{EV}$.

TIC,

1984. 6P. MN (LA-UR-84-0720)

KANIA, DON R. (P-14)

BARTLETT, ROGER J. (P-14)

TRELA, WALTER J. (P-DO)

SPIUER, EBERHARD

GOLUB, L.

49-109

PHYSICS DIVISION ANNUAL PROGRESS

REPORT-JANUARY 1-DECEMBER 31, 1983.

LOS ALAMOS

1984. 96P. (LA-10097-PR)

TRELA, WALTER J. (P-14)

49-110

PION INELASTIC SCATTERING TO THE FIRST

THREE EXCITED STATES OF LITHIUM - 6.

LOS ALAMOS,

1984. 176P. (LA-10257-T)

KIZIAH, R. R.

49-111

PLASMA ENERGY DEPOSITION FROM THE NUCLEAR ELASTIC SCATTERING OF TRITONS

ON TRITONS

LOS ALAMOS.

1984. 6P. (LA-10042-MS)

DEVANEY, JOSEPH J. (X-6) 
49-112

PLASTIC INSTABILITY AND PARTICULATION IN STRETCHING METAL JETS.

J. APPL. PHYS.,

V.56,P.1997-2006. 1984.

WALSH, JOHN M. (M-4)

49-113

PRECISE ULTRAHIGH - PRESSURE

EXPERIMENTS

TIC.

1983. 5P. MN (LA-UR-83-2081)

RACAN, CHARLES E. (P-3)

DIVEN, BENJAMIN C. (P-DO)

TEASDALE, WILLIAM A. (P-3)

ROBINSON, EDWARDE. (P-3)

RICH, MARVIN (X-5)

49-114

PREDICTION OF HIGH PRESSURE PHASE

TRANSITIONS IN SOLID N2 AND THE PRESSURE

DEPENDENCE OF THE INTRAMOLECULAR

VIBRATIONAL.FREQUENCIES.

TKC.

1983. 6P. MN (LA-UR-83-2249)

ETTERS, RICHARD D. (M-6)

CHANDRASEKHARAN, $V$.

KOBASHI, $K$

49-115

PREDICTION OF PRESSURE INDUCED STRUCTURAL PHASE TRANSTTIONS AND INTERNAL MODE FREQUENCY CHANGES IN SOUD NITROGEN 2.

TIC.

1883. 5P. MN (LA-UR-2146)

ETTERS, RICHARD D. (M-6)

KOBASH, $K$.

CHANDRASEKHARAN, $V$.

49-116

PROCEEDINGS OF THE ITTH LAMPF USERS

GROUP MEETING, LOS ALAMOS, NM,

NOVEMBER 7 - 8, 1983

LOS ALAMOS,

1984. 176P. (LA-10080-C)

BRADBURY, JAMES N. (MP-DO)

RUMINER, KIT (MP-DO)

TALLEY, BEVERLY (MP-DO)

49-117

PROCEEDINGS OF THE 1984 WORKSHOP ON HIGH-ENERGY EXCITATIONS IN CONDENSED MATTER-HELD AT LOS ALAMOS, NEW MEXICOFEBRUARY 13-15, 1984.

LOS ALAMOS.

1984. 343P. (LA-10227-C, VOL. 1) SILVER, RICHARD N. (P-6)

49-118

PROCEEDINGS OF THE 1984 WORKSHOP ON HIGH-ENERGY EXCITATIONS IN CONDENSED MATTER-HELD AT LOS ALAMOS, NEW MEXICOFEBRUARY 13-15, 1984.

LOS ALAMOS

1984. 651P. (LA-10227-C, VOL 2)

SILVER, RICHARD N. (P-8)
49-119

PROCRESS IN ULTRAVIOLET DAMAGE TESTING AT LOS ALAMOS.

LASER INDUCED DAMAGE IN OPTICAL

MATERIALS, 14TH, 1982, BOULDER.

PROC., P.266-81. NBS, 1984.

(620.112/S9890M/14TH/1982)

ALSO PUBLISHED IN:

TIC,

1982. 9P. MN (LA-UR-83-0601)

FOLTYN, STEPHEN R, (CHM-5)

JOUN, L. J. (CHM-5)

NEWNAM, BRIAN E. (CHM-6)

49-120

PULSED-POWER DIODE GENERATION OF HIGH - POWER MICROWAVES

TIC,

1983. 5P. MN (LA-UR-83-2622)

PERATT, ANTHONY L. $(X-8)$

MOSTROM, MICHAEL A. $(X-8)$

KWAN, THOMAS J. T. $(\alpha-8)$

THODE, LESTER E. $(X-8)$

49-121

QUANTUM MANIFESTATIONS OF CLASSICAL RESONANCE ZONES.

J. CHEM. PHYS.

V.80, P.794-9. 1984.

DE LEON, NELSON (T-12)

DAVIS, MICHAEL $J$.

HELLER, ERIC J. (T-12)

49-122

RARE MUON DECAYS AND LEPTON - FAMILY NUMBER CONSERVATION

TIC.

1984. 30P. MN (LA-UR-84-1327)

HOFFMAN, CYRUS M. (MP-4)

49-123

RAREFACTION VELOCITIES IN SHOCKED

TANTALUM AND THE HIGH PRESSURE MELTINC POINT.

TIC,

1983. 5P. MN (LA-UR-83-2144)

BROWN. J. MICHAEL

SHANER, J.W. (M-6)

49-124

REFLECTED SHOCKS IN SILICON DIOXIOE.

TIC.

1983. 5P. MN (LA-UR-83-2242)

FRITZ, JOSEPHN. (M-6)

MC QUEEN, ROBERT G. (M-6)

49-125

RELAXATIONS IN SPIN GLASSES -

SIMILARTIES AND DIFFERENCES FROM

ORDINARY GLASSES.

TIC,

1983. 13P. MN (LA-UR-83-2929)

NGAl, K. L.

RAJAGOPAL, A.K

HUANG, CHAO YUAN (P-10)
49-126

RESPONSE TO AMBIENT PRESSURE OF FUEL

DROP COMBUSTION WITH SURFACE

EQUIUBRIUM.

INT. J. ENG. SCl.

V.22, P.403-10. 1984

JANSSEN, ROBERT D. (CNLS)

LUDFORD, G. S. S.

49-127

REVISION OF THE STATISTICAL MECHANICS OF PHONONS TO INCLUDE PHONON LUNE WIDTHS.

TIC.

1983. 4P. MN (LA-UR-83-2379)

OVERTON, WILLIAM C., JR. (P-10)

49-128

ROLE OF PRESSURE IN UNDERSTANDING THE

ANOMALOUS SUPERCONDUCTIVITY IN

EUROPIUM (MOLYBDENUM)G(SULFURIS AND

(DITETRAMETHYLTETRASELENAFULVALENIUM)

FLUOROSULFTE

TIC.

1983. 11P. MN (LA-UR-83-2451)

WOUF, S. A.

HUANG, CHAO YUAN (P-10)

LACOE, RONALD C.

CHAIKIN, PAUL M.

FULLER, WENDY WEBB

WUO, HUEY LIN

WUDL, FRANK

49-129

SCATTERING OF RAYLEIGH SURFACE WAVES

FROM PARTLY - CLOSED SURFACE - BREAKING

CRACKS

REVIEW OF PROGRESS IN QUANTITATIVE

NONDESTRUCTIVE EVALUATION, 1983

BOULDER. PROC., P.187-97. PLENUM,

1984.

VISSCHER, WILLAM MAURITS (T-11)

49-130

SHAPE RESONANCES IN THE HYDROGEN STARK

EFFECT IN FIELDS UP TO 3 MV/CM.

PHYS. REV. LETT.

V.53, P.775-8. 1984.

BERGEMAN, $T$.

HARVEY, C.

BUTTERFIELD, KENNETH B. (Q-2)

BRYANT, HOWARD C.

CLARK, DAVD A. (P-9)

GRAM, PETER A. M. (MP-4)

MAC ARTHUR, DUNCAN W

DAVIS, M. (MP-T)

DONAHUE, JOEY B. (MP-7)

DAYTON, $J$.

SMITH, W. W.

49-131

SHEAR VISCOSITY OF THE HARD-SPHERE

FLUID VIA NONEQUIUBRIUM MOLECULAR

DYNAMICS.

PHYS. REV. LETT.,

V.52, P.1333-5. 1984

ERPENBECK, JEROME J. (T-11) 
49-132

SHOCK-WAVE EXPERIMENTS AT

THREEFOLD COMPRESSION.

PHYS. REV.

V.29A, P.1391-1401. 1984

RAGAN, CHARLES E. (P-3)

49-133

SIMPLE EOS FOR LINEAR

POLYTETRADEUTEROETHYLENE.

LOS ALAMOS.

1984. 14P. (LA-10241-MS)

DOWELL, FLONNIE $(T-4)$

49-134

SIMPLE EOS FOR THE SILICONE RUBBER

SYLGARD 184.

LOS ALAMOS,

1984. 15P. (LA-10164-MS)

DOWELL, FLONNIE (T-4)

49-135

SIMULATION STUDIES ON A NOVEL BETATRON INJECTION SCHEME

TIC,

1983. 5P. MN (LA-UR-83-2669)

PETER, WILLIAM K. $(X-8)$

FAEHL, R. J. (X-8)

MAKO, F.

49-136

SIMULATIONS OF THE EFFECTS OF MOBILE IONS ON THE RELATIVISTIC BEAM - PLASMA INSTABILITY FOR INTENSE BEAMS.

TIC.

1983. 5P. MN (LA-UR-83-2601)

JONES, MICHAEL E. $(X-8)$

LEMONS, DON S. $(X-B)$

LEE, HUAN $(X-8)$

49-137

SINGLE - PULSE COHERENT RAMAN SPECTROSCOPY IN SHOCK - COMPRESSED BENZENE.

MATERIALS RESEARCH SOCIETY SYMPOSIA,

1983, BOSTON. PROC., P.87-94.

ELSEVIER, 1984.

ALSO PUBLISHED IN:

TIC

1983. 9P. MN (LA-UR-63-1854)

MOORE, DAVID S. (CHM-4)

SCHMIDT, STEPHEN C. (M-6)

SCHIFERL, DAVID (M-6)

SHANER, J. W. (M-6)

49-138

SOME OBSERVATIONS ON HEAVY FERMION

SUPERCONDUCTIVITY IN URANIUM

BERYLLIUM 13

J. LOW TEMP. PHYS.

V.56, P.379-81. 1984

STEWART, GREGORY R. (MST-5)

GIORGI, ANGELO L. (MST-5)
49-139

SPACE TIME EVOLUTION OF THE NONLINEAR

TWO - STREAM INSTABILITY.

TIC,

1983. 5P. MN (LA-UR-83-2670)

LEMONS, DON S. (X-8)

JONES, MICHAEL E. $(X-8)$

LEE, HUAN $(X-8)$

49-140

SPECIFIC HEAT STUDIES OF LANTHANUM AND YTTRIUM SESQUICARBIDES.

J. LOW TEMP. PHYS.,

V.54,P.149-53. 1984.

CORT, BARBARA (MST-3)

STEWART, GREGORY R. (MST-5)

GIORGI, ANGELOL. (MST-5)

49-141

SPEED AND ATTENUATION OF ELASTIC WAVES

IN POLYCRYSTALS

REVIEW OF PROGRESS IN QUANTITATIVE

NONDESTRUCTIVE EVALUATION, 1983,

BOULDER. PROC., P.1149-55. PLENUM,

1984.

BRIND, R. J.

GUBERNATIS, JAMES E. (T-11)

49-142

SPIDER DIFFRACTION, A COMPARISON OF

CURVED AND STRAIGHT LEGS.

APPL. OPT.

V.23, P.1907-13. 1984

RICHTER, JOHN L. (NSP-WP)

49-143

SPOTSIZE EFFECTS IN LASER DAMAGE

TESTING.

LASER INDUCED DAMAGE IN OPTICAL

MATERIALS, 14TH, 1982, BOULDER.

PROC., P.368-79. NBS, 1984.

(620.112/S989OM/14TH/1982)

ALSO PUBLISHED IN:

Tic,

1982. 12P. MN (LA-UR-83-1020)

FOLTYN, STEPHEN R. (CHM-5)

49-144

STABILTY OF LANGMUIR WAVES IN

DEVELOPING ION - SOUND TURBULENCE.

PHYS. FLUIDS

V.27, P.218-27. 1984

DUBOIS, DONALD F. $(T-11)$

PESME, DENIS

49-145

STARK BROADENING OF HYDROGEN NEGATIVE

RESONANCES IN LARGE ELECTRIC FIELDS

LOS ALAMOS,

1984. 158P. (LA-10149-T)

BUTTERFIELD, KENNETH B. (Q-2)
49-146

STRAIGHT MAN FOR NONLINEARITY

AN INTERVIEW WITH ALWYN SCOTT.

LOS ALAMOS SCI.

SPRING, NO.10, P.70-80. 1984.

LATHROP, JUDITH M. (IS DO)

49-147

STRUCTURAL STUDIES AT ELEVATED PRESSURE AND REDUCED TEMPERATURES USING

SYNCHROTRON RADIATION - APPLICATION TO (CERIUM).8(LANTHANUM).1(THORIUM).1.

TIC

1983. 6P. MN (LA-UR-83-2928)

SKELTON, E. F.

WEBB, A. W.

ELAM, WEST T.

WOLF, S. A

OADRI, SYED B.

HUANG, CHAO YUAN (P-10)

CHAIKIN, PAUL M.

LACOE, RONALD C.

GSCHNEIDER, KARL A.

49-148

STUDY OF DYNAMICAL LIGHT SCATTERING IN PHASE SEPARATING HELIUM 3 - HELIUM 4 MIXTURES USING LINEAR PHOTODIODE ARRAYS.

REV. SCI. INSTRUM.

V.55, P.875-8. 1984

SINHA, DIPEN N. (P-10)

HOFFER, JAMES K. (P-10)

$49-149$

SUPERCONDUCTIVITY AND SPIN

FLUCTUATIONS.

LOS ALAMOS SCI.,

SPRING, NO.10, P.60-1. 1984

PINES, DAVID

49-150

SUPERSYMMETRY BREAKING WITHOUT

O'RAIFEARTAIGH POTENTIALS.

PHYS. LETT.,

V.134B, P.51-5. 1984

OVRUT, BURT A.

RABY, STUART A. (T-8)

49-151

THERMAL CONDUCTIVITY OF AN ARBITRARILY

DENSE PLASMA.

LOS ALAMOS

1984. 39P. (LA-10202-MS)

RINKER, GEORGE A., JR. (T-4)

49-152

THERMODYNAMIC CALCULATIONS FOR BCC

SODIUM AT HIGH PRESSURES.

PHYS. REV.

V.30B, P.3929-32. 1984.

STRAUB, GALEN K. (T-4)

WALLACE, DUANE C. $(X-4)$ 
49-453

TRANSMISSION AND REFLECTION OF LASER BEAMS PROPAGATING THROUGH AEROSOL

CLOUDSIN SPACE.

LOS ALAMOS,

1984. 38P. (LA-09998-MS)

ZARDECKI, ANDREW (T-DO)

GERSTL, S. A. W. (T-DOT)

49-154

TWO - PHASE FLUID FLOW THROUGH NOZZLES AND ABRUPT ENLARGEMENTS.

TIC.

1983. 45P. MN (LA-UR-63-3695)

OLIA, $H$.

MAEDER, P. F.

DI PIPPO, R.

DICKINSON, D. A.

49-155

UNDERSTANDING EXPERIMENTALLY THE

TRANSITION TO CHAOS IN A CONVECTING

DILUTE SOLUTION OF 3 HELIUM IN

SUPERFLUID 4 HELIUM.

QUANTUM FLUIDS AND SOLIDS-1983,

1983, SANIBEL ISLAND. PROC.,

P.412-27. AIP, 1983.

(530.4/Q1FL/1983)

ALSO PUBLISHED IN:

TIC.

1983. 22P. MN (LA-UR-83-1252)

MAENO, YOSHITERU

HAUCKE, HANS

WHEATLEY, JOHN C. (P-10)
49-156

URANIUM 2 PLATINUM CARBIDE 2 AND SYSTEMATICS OF HEAVY FERMIONS.

PHYS. REV. LETT.,

V.53, P.1829-31. 1984.

MEISNER, G. P. (MST-5)

GIORGI, ANGELO L. (MST-5)

LAWSON, A. C. (MST-5)

STEWART, GREGORY R. (MST-5)

WILUS, JEFFREY O. (MST-5)

WIRE, MIKE S. (MST-5)

SMITH, JAMES L. (MST-5)

49-157

USE OF FIBER OPTICS AND LASER - INDUCED FLUORESCENCE FOR REMOTE MEASUREMENTS OF URANIUM HEXAFLUORIDE IN STRONG ROTATION.

TIC.

1983. 32P. MN (LA-UR-83-1699)

ALLISON, S.W.

MAGNUSON, D. W.

DAVIES, T.J.

CATES, MICHAEL REID (0-1)

GENTRY, RICHARD A. (T-3)

CALOWELL, STEPHEN E. (P-14)

FRANKS, L. A.

NELSON, M. A.

49-158

USING THE SURFACE TENSION TO ESTIMATE THE CONDENSATE DENSITY OF SUPERFLUID

4 HELIUM.

QUANTUM FLUIDS AND SOLIDS-1983,

1983, SANIBEL ISLAND. PROC.,

P.403-11. AIP, 1983

(530.4/Q1FL/1983)

ALSO PUBLSSHED IN:

TIC,

1983. 17P. MN (LA-UR-83-1070)

CAMPBELL, LAURENCE J. (P-10)
49-159

$X$-RAY BIOMICROHOLOGRAPHY.

OPT. ENG.

V.23, P.193-203. 1984.

SOLEM, JOHNDALE C. (T-DO)

CHAPLINE, GEORGEF.

49-160

XUVMUV FREE - ELECTRON LASER

OSCILLATOR.

LASER TECHNIQUES IN THE EXTREME

ULTRAVIOLET, 1984, BOULDER. PROC.,

P.293-303. AIP, 1984.

(535.84/T674LA/2ND/1984)

ALSO PUBLISHED IN:

TIC,

1984. 12P. MN (LA-UR-84-1384)

GOLDSTEIN, JOHN C. (X-1)

NEWNAM, BRIAN E. (CHM-6)

COOPER, RICHARD K. (AT-6)

COMLY, JACK C., JR. (X-1)

$49-161$

YANG-MILLS MAGNETOHYDRODYNAMICS NONRELATIVISTIC THEORY.

PHYS. REV.

V.30D, P.2557-60. 1984.

HOLM, DARRYL D. (CNLS)

KUPERSHMIDT, BORIS ABRAM (CNLS) 


\section{PHYSICS-NUCLEAR}

50-1

ANALYSIS OF INTERMEDIATE STRUCTURE IN THE FISSION AND CAPTURE CROSS SECTIONS OF (235 URANIUM PLUS NEUTRON)

PHYS. REV.

V.30C, P.214-6. 1984.

MOORE, MICHAEL S. (P-3)

CALABRETTA, L

CORVI, FRANCO

WEIGMANN, $\mathrm{H}$.

50-2

ANALYTICAL APPLICATIONS FOR DELAYED NEUTRONS.

TIC,

1883. 13P. MN (LA-UR-83-3058)

ECCLESTON, GEORGE W. (Q-1)

$50-3$

ANALYZING POWER MEASUREMENTS FOR

3 HELIUM (PROTON, PROTON) 3 HELUM

ELASTIC SCATTERING BETWEEN 20 AND

50 MEV.

PHYS. REV.

V.29C. P. 2009-12. 1984

BIRCHALL, J.

VAN OERS, WILLEM T. H.

WATSON, J.

CONZETT, H.E.

LARIMER, R. M.

LEEMANN, $B$.

STEPHENSON, E.J.

VON ROSSEN, $P$.

BROWN, RONALD E. (P-3)

$50-4$

ANGULAR DISTRIBUTION FOR NITROGEN 15

(PI PLUS, P ZERO) OXYGEN 15 (G.S.)

AT T SUB PI EQUALS 48 MEV.

48 MEV.

PHYS. REV. LETT.

V.52, P.1100-3. 1984

COOPER, MARTIN D. (MP-4)

BAER, H.W. (MP-4)

BOLTON, RICHARD D. (MP-4)

BOWMAN, J. DAVID (MP-4)

CVERNA, FRANK H. (P-15)

KING, N. S. P. (MP-4)

LEITCH, M. J. (MP-4)

ALSTER, JONAS

DORON, ALEX

ERELL, A.

MOINESTER, MURRAY A.

BLACKMORE, EW W.

SICILIANO, EDWARD R.
50-5

ANNIHILATION OF LOW - ENERGY

ANTIPROTONS IN NUCLEI.

PHYSICS AT LEAR WITH LOW-ENERGY COOLED ANTIPROTONS, 1982, ERICE. PROC., P.543-60. PLENUM, 1984.

(539.72/W926PH/1982)

ALSO PUBLISHED IN:

TIC,

1982. 19P. MN (LA-UR-82-2540)

DE VAIES, RALPH M. (P-T)

DIGIACOMO, NICHOLAS J. (P-3)

$50-6$

ANTIPROTON - NUCLEUS INTERACTION.

TIC,

1984. 11P. MN (LA-UR-84-1872) GIBBS, WILLIAM R. (T-5)

50-7

APPLED NUCLEAR DATA RESEARCH AND

DEVELOPMENT SEMIANNUAL PROGRESS REPORT,

APRIL 1 - SEPTEMBER 30, 1983.

LOS ALAMOS,

1984. 103P. (LA-10069-PR)

ARTHUR, EDWARD D. (T-2)

$50-8$

CALCULATION OF NUCLEAR PROPERTIES FAR

FROM THE LINE OF BETA - STABILTYY.

TIC.

1983. 6P. MN (LA-UR-83-2355)

KRUMLINDE, J.

MOUER, PETER

NIX, JAMES R. (T-9)

$50-9$

CAN WE LEARN ABOUT THE SPIN FUP

GIANT DIPOLE RESONANCES WITH PIONS?

INTERNATIONAL CONFERENCE ON SPIN

EXCITATIONS IN NUCLEI, 1982

TELLUURIDE. PROC., P.523-37. PLENUM 1984. (539.7/161SP/1982)

ALSO PUBLISHED IN:

TIC.

1982. 17P. MN (LA-UR-82-1567)

BAER, H. W. (MP-4)

-50-10

CARBON 13 (PROTON,PROTON) CARBON 13

REACTION AT TP EQUALS 547 MEV.

PHYS. REV.,

V.30C, P.270-8. 1984

SEESTROM-MORRIS, SUSAN J.

FRANEY, M.A.

DEHNHARD, DIETRICH

HOLTKAMP, DAVID B. (AT-2)

BOUDRIE, RICHARD L. (MP-10)

AMANN, JAMES F. (MP-10)

IDZOREK, G. C. (MP-10)

GOULDING, CHARLES A.
50-11

CHARACTERISTIC DIRAC SIGNATURE IN

ELASTIC PROTON SCATTERING AT

INTERMEDIATE ENERGIES.

PHYS. REV. LET.,

V.52, P.978-81. 1984.

HYNES, M. V. (MP-4)

PICKLESIMER, A

TANDY, P.C.

THALER, R. M.

50-12

CHARGE SYMMETRY IN PION - DEUTERON

ELASTIC SCATTERING.

PHYS. REV.,

V.30C, P.2010-20. 1984

MASTERSON, T. G.

KRAUSHAAR, J.J.

PETERSON, R. J.

RAYMOND, R.S.

RISTINEN, R. A.

ULLMANN, J.L.

BOUDRIE, RICHARD L. (MP-10)

GILL, D. R.

GIBSON, E. F.

THOMAS, A.W.

50-13

COMPARISON OF APPROXIMATE

CHIRAL - DYNAMICAL PI NEUTRON GOING TO

PI PI NEUTRON MODELS USED IN

A(PI,2PI) CALCULATIONS.

PHYS. REV.

V.30C, P.224-30. 1984

BHALERAO, R. S. (INC-11)

LIU, LON CHANG (INC-11)

50-14

COMPARISON OF SPIN - GLASS DYNAMICS

DETERMINED BY THE MUON-SPIN-

RELAXATION AND NEUTRON SPIN-ECHO

TECHNIQUES.

PHYS. REV.,

V.29B, P.6048-51. 1984.

HEFFNER, ROBERT H. (MP-3)

MAC LAUGHLN, DOUGLASE 
50-15

COMPLETE MEASUREMENT OF POLARIZATIONTRANSFER OBSERVABLES FOR THE 12 CARBON (P,P') 12 CARBON REACTION AT 500 MEV.

PHYS. REV. LETT.

V.52, P.98-101. 1984

MC CLELLAND, JOHN B. (MP-10)

MOSS, J. (MP-10)

AAS, BJORNE

AZIZI, A.

BLESZYNSKI,E.

BLESZYNSKI, $M$.

GEAGA, J.

IGO, G.

RAHBAR, A.

WAGNER, J. $B$.

WESTON, GARY S

WHITTEN, C.

JONES, $K$.

NANDA, SIRISH

GAZZALY, MAGDI

HINTZ, N.

60-16

CONTRIBUTIONS OF DOUBLE - DELTA AND OTHER TWO-NUCLEON MECHANISMS TO PIONIC PION PRODUCTION ON THE DEUTERON.

TIC.

1983. 8P. MN (LA-UR-83-1397)

LIU, LON CHANG (INC-11)

BHALERAO, R. S. (INC-11)

PIASETZKY, ELI

50-17

CONTROL MATERIALS AND LIGHT COOLANT

CROSS - SECTION DATA.

PROG. NUCL. ENERGY,

V.13, P.193-233. 1984

YOUNG, PHILLIP G., JR. (T-2)

STEWART, LEONA (T-2)

50-18

CORE EXCITATION EFFECTS IN PION DOUBLE CHARGE EXCHANGE

LOS ALAMOS,

1984. 132P. (LA-09947-T)

KALETKA, $M$.

$50-18$

CORE-EXCITATION EFFECTS IN PION DOUBLE

CHARGE EXCHANGE.

PHYS. REV. LETT.

V.52, P, 894-7.1984.

SETH, KAMAL $K$.

KALETKA, M.

IVERSEN, STEVEN

SAHA, A.

BARLOW, D.

SMITH, D.

LU, LON CHANG (INC-11)
$60-20$

CROSS SECTIONS AND MAXWELLIAN REACTION

RATES FOR POLARIZED DEUTERON PLUS

DEUTERON REACTIONS

LOS ALAMOS

1984. 19P. (LA-09971-MS)

HALE, GERALD M. (T-2)

DOOLEN, GAFY D. $(X-5)$

50-21

D SS, D LL, D SL, D LS, AND P FOR PP

COING TO PP AT 600 TO 800 MEV.

PHYS. REV.

V.30C, P.1251-5, 1984

HOLLAS, C.L.

CREMANS, D.J.

MC NAUGHTON, K. H.

RILEY,P.J.

RODEBAUGH, R.F

XU, SHEN-WU

BONNER, BILLY E. (P-3)

MC NAUGHTON, MICHAEL W. (MP-13)

OHNUMA, H.

VAN DYCK, OLIN B. (MP-13)

TSU-HSUN, SUN (MP-10)

TURPIN, S. E

AAS, BJORNE

WESTON, GAFY $S$.

50-22

DELTA - THREE - THREE DYNAMICS IN PION

DOUBLE CHARGE EXCHANGE.

PHYS. REV. LETT.,

V.52, P.593-6. 1984

JOHNSON, MIKKEL B. (MP-DO)

SICILIANO, EDWARD R

TOKI, $H$.

WIRZBA, A.

$50-23$

DELTA THREE - THREE RESONANCE IN PION NUCLEUS ELASTIC, SINGLE AND DOUBLE CHARGE EXCHANGE SCATTERING.

TIC.

1983. 28P. MN (LA-UR-83-1725)

JOHNSON, MIKKEL B. (MP-DO)

$50-24$

DEPENDENCE OF THE EXCITATION ENERGY, WIDTH, AND CROSS SECTION OF THE ISOVECTOR MONOPOLE RESONANCE

TIC,

1983. 6P. MN (LA-UR-83-2927)

BOWMAN, J. DAVD (MP-4)

BAER, H. W. (MP-4)

COOPER, MARTIN D. (MP-4)

MATIS, H. S. (MP-4)

IROM, FAROKH

PIASETZKY, ELI

SENNHAUSER, URS (MP-4)

KING, N. S. P. (P-15)

ALSTER, JONAS

ERELL, A.

MOINESTER, MURRAYA

LICTENSTADT, J.

INGRAM, $\mathrm{O}$
$50-25$

DETERMINATION OF THE CROSS SECTION FOR

159 TERBIUM (NITROGEN, 2 NITROGEN) 158

TERBIUM AND THE HALF - LIFE OF 158

TERBIUM.

PHYS. REV.

V.30C, P.623-5. 1984.

PRESTWOOD, RENE J. (INC-11)

CURTIS, DAVIO B. (INC-7)

ROKOP, DONALD J. (INC-7)

NETHAWAY, DAVID R.

SMITH, NORMANL.

50-26

DEUTERON ELECTRIC POLARIZABILTYY.

PHYS. REV.

V.29C, P.232-9. 1984

FRIAR, JAMES L. (T-5)

FALLIEROS, $\mathbf{S}$.

60-27

DEUTERON FORWARD PHOTODISINTEGRATION-

MESON CURRENTS AND RELATIVITY.

PERSPECTIVES IN NUCLEAR PHYSICS AT

INTERMEDIATE ENERGIES, 1983, TRIESTE

PROC., P.124-51. WORLD SCIENTIFIC

PUBL., 1984. (539.7/P467NU/1983)

FRIAR, JAMES L. (T-5)

50-28

DEUTERON FORWARD PHOTODISINTEGRATION.

PHYS. REV

V.30C. P.441-5. 1984.

ALSO PUBLISHED IN

TIC.

1983. 16P. MN (LA-UR-83-2763)

FRIAR, JAMES L. (T-5)

GIBSON, BENJAMIN F. (T-5)

PAYNE, GERALDL

$50-29$

DOUBLE BETA DECAY.

PROG. PART. NUCL: PHYS.,

V.12, P.409-75. 1984.

HAXTON, WICK C. (T-5)

STEPHENSON, GERALD J., JR. (T-5)

$60-30$

DYNAMICS OF FISSION AND HEAVY ION

REACTIONS.

NUCL. PHYS.

V.A428, P.161-74. 1984

NIX, JAMES R. (T-9)

SIERK, ARNOLD J. (T-9)

$50-31$

ELASTIC AND INELASTIC SCATTERING OF

POLARIZED PROTONS FROM CARBON 12 AT

400,600, AND 700 MEV.

LOS ALAMOS,

1984. 318P. (LA-10064-T)

JONES, K. W. (MP-10) 
$50-32$

ELASTIC SCATTERING OF POLARIZED TRITONS FROM CARBON 12, VANADIUM 51, AND 58 NICKEL AT 9 AND 11 MEV.

PHYS. REV.

V.29C, P.324-6. 1984.

FICK, D.

BROWN, RONALDE. (P-3)

GRUEBLER, WILLI (P-DOR)

HARDEKOPF, ROBERT A. (AT-3)

HANSPAL, J.S.

50-33

ELECTROMAGNETIC AND WEAK INTERACTIONS IN FEW - NUCLEON SYSTEMS.

TIC,

1983. 17P. MN (LA-UR-83-2432)

GIBSON, BENJAMIN F. (T-5)

$50-34$

ENERGIES OF THE MUONIC L AND M

TRANSITIONS OF THE EVEN-A LEAD

ISOTOPES.

PHYS. REV.

V.30C, P.704-7. 1984

HOEHN, MARTHA V. (MP-13)

SHERA, E. BROOKS (P-3)

$50-35$

EVALUATION AND TESTING OF N PLUS

239 PLUTONIUM NUCLEAR DATA FOR REVISION

2 OF ENDF/B-V.

NUCL. SCl. ENG.,

V.88, P.56-70. 1984

ARTHUR, EDWARD D. (T-2)

YOUNG, PHILLIP G., JR. (T-2)

MADLAND, DAVID G. (T-2)

MAC FARLANE, ROBERT E. (T-2)

50-36

EVIDENCE FOR DELTA - HOLE COMPONENTS

FROM PION REACTIONS.

INTERNATIONAL CONFERENCE ON SPIN

EXCITATIONS IN NUCLEI, 1982

TELLURIDE. PROC., P.161-71. PLENUM, 1984. (539.7/161SP/1982).

MORRIS, CHRISTOPHER L. (MP-10)

$50-37$

EXCITATION FUNCTION OF PION SINGLE

CHARGE EXCHANGE REACTIONS IN

ALUMINUM 27, SCANDIUM 45, AND COPPER 65.

PHYS. REV.

V.30C, P.1597-1602. 1984.

RUNDBERG, ROBERT S. (INC-11)

DROPESKY, BRUCE J. (CNC-11)

GIESLER, GREGG CARL (INC-11)

BUTLER, GILBERT W. (INC-11)

KAUFMAN, SHELDON B.

STEINBERG, E.P.

50-38

EXCITATION OF GIANT RESONANCES IN

PION CHARGE - EXCHANGE REACTIONS.

LOS ALAMOS,

1984. 18P. (LA-09928-MS)

AUERBACH, NAFTALI
$50-39$

EXCITATION OF GIANT RESONANCES IN PION

CHARGE - EXCHANGE REACTIONS.

TIC,

1983. 16P. MN (LA-UR-83-2587)

AUERBACH, NAFTALI

$50-40$

EXISTENCE OF FINITE-ACTION SOLUTIONS

TO THE COLEMAN-DE LUCCIA EQUATIONS

PHYS. REV.

V.29D, P.773-4. 1984

MOTTOLA, EMIL

LAPEDES, ALAN S. (T-6)

$50-41$

EXPERIMENTAL STUDY OF INCLUSIVE PION DOUBLE CHARGE EXCHANGE REACTIONS IN

THE DELTA RESONANCE REGION.

LOS ALAMOS,

1984. 246P. (LA-09932-T)

WOOD, S. A.

$50-42$

EXTENDED SIEGERT THEOREM.

PHYS. REV.,

V.29C, P.1645-55. 1984.

FRIAR, JAMES L. (T-5)

FALLIEROS, STAVROS L

$50-43$

FEW BODY EXPERIMENTS WITH POLARIZED

BEAMS AND POLARIZED TARGETS.

TIC,

1983. 13P. MN (LA-UR-83-2653)

SIMMONS, JAMES E. (P-7)

$50-44$

FIELD COMPENSATION AS AN ALTERNATIVE TO

MAGNETIC SHIELDING IN SEARCHES FOF

NEUTRON - NEUTRON TRANSITIONS.

PHYS. REV.

V.290, P.1537-8. 1984.

KABIR, P. (T-5)

NUSSINOV, S. (T-8)

AHARONOV, $Y$.

50-45

FISSION CROSS SECTIONS UP TO $20 \mathrm{MEV/}$

NUCLEON.

PHYS. REV.,

V.30C, P.1550-60. 1984.

GAVRON, AVIGDOR I. (P-3)

BOISSEVAIN, JAN G. (P-3)

BRITT, HAROLD C. (P-3)

ESKOLA, KARI A. (INC-11)

ESKOLA, PIRKKO L. (INC-11)

FOWLER, MALCOLM M. (INC-7)

OHM, HENNER (P-3)

WILHELMY, JERRY B. (INC-11)

AWES, T.

FERGUSON, R. L

OBENSHAIN, F. E.

PLASIL, F.

YOUNG, G. R.

WALD, $\mathbf{S}$.
$50-46$

FISSION OF 255,256 EINSTEINIUM

255-257 FERMIUM AND 258 MENDELEVIUM

AT MODERATE EXCITATION ENERGIES.

PHYS. REV.

V.30C, P.559-65. 1984

BRITT, HAROLD C. (P-3)

HOFFMAN, DARLEANE C. (NC-DO)

VAN DER PLICHT, J. (P-3)

WILHELMY, JERRY B. (INC-11)

CHEIFETZ, ELI (P-9)

DUPZYK, RONALD J.

LOUGHEED, R. W.

$50-47$

FORWARD - ANGLE PION INELASTIC

SCATTERING.

LOS ALAMOS,

1984. 218P. (LA-09960-T)

BLAND, L. C.

$50-48$

FUSION-ENERGY REACTION

HYOROGEN T,ALPHAN FROM ET EQUALS

12.5 TO $117 \mathrm{KEV}$

PHYS. REV.

V.29C, P.2031-45. 1984.

JARMIE, NELSON (P-3)

BROWN, RONALOE. (P-3)

HARDEKOPF, ROBERT A. (AT-3)

$50-49$

GAMOW - TELLER STRENGTH FUNCTIONS AND

NEUTRINO PROBLEMS.

INTERNATIONAL CONFERENCE ON SPIN

EXCITATIONS IN NUCLEI, 1982,

TELLURIDE. PROC., P.605-24. PLENUM

1984

ALSO PUBLISHED IN:

TIC,

1982. 24P. MN (LA-UR-82-1627)

HAXTON, WICK C. (T-5)

$50-50$

GENERALIZED CRANKING MODEL FOR

COLLECTIVE NUCLEAR MOTION.

NUCL. PHYS.,

V.A426, P.353-78. 1984

KUNZ, JUTTA (T-9)

NIX, JAMES R. (T-9)

50-51

GROUND - STATE AND TRANSITION CHARGE

DENSITIES IN OSMIUM 192.

PHYS. REV.,

V.30C, P.1465-78. 1984.

REUTER, WILFRIED (P-3)

SHERA, E. BROOKS (P-3)

HOEHN, MARTHA V. (MP-13)

HERSMAN, F. W.

MILLIMAN, T.

FINN, J.

HYDE-WRIGHT, C

LOURIE, $R$.

PUGH, $\mathbf{B}$.

BERTOZZI, $W$. 
50.52

HALF LIFE OF PLUTONIUM-240 DETERMINED BY SPECIFIC-ACTIVITY MEASUREMENTS.

INT. J. APPL. RADIAT. ISOT.

V.35, P.171-2. 1984.

STEINKRUGER, FREDERICK J. (CMB-1)

MATLACK, GEORGE M. (CMB-1)

BECKMAN, RICHARD J. (S-1)

50-53

HALF LIFE OF 26 ALUMINUM.

TIC,

1983. 9P. MN (LA-UR-83-1384)

NORRIS, THOMAS L. (INC-7)

GANCARZ, ALEXANDER J. (CNC-11)

ROKOP, DONALD J. (INC-7)

THOMAS, KIMBERLY W. (INC-11)

50-54

HE - JET SYSTEM TO STUDY SHORT - LIVED FISSION PRODUCT NUCLEI AT LAMPF.

TIC.

1983. 10P. MN (LA-UR-83-3061)

TALBERT, W. L., JR. (INC-11)

BUNKER, MERLE E. (INC-5)

STARNER, JOHN W. (INC-5)

50-55

HIGH - ENERGY GAMMA - RAY DETECTOR SYSTEM FOR FAST NEUTRON - INDUCED

REACTIONS.

NUCL. INSTRUM. METHODS,

V.220, P.371-6. 1984

WENDER, STEVE A (P-3)

AUCHAMPAUGH, GEORGE F. (P-3)

WILKERSON, J. F. (P-3)

HILL, N. W.

NILSSON, L. R.

ROBERSON, N. R.

50-56

HIGH ENERGY NUCLEAR STRUCTURES.

TIC,

1984. 5P. MN (LA-UR-84-0823)

BOGUTA, J.

KUNZ, JUTTA (T-9)

50-57

HIGH NUCLEAR TEMPERATURES BY

ANTIMATTER - MATTER ANNIHILATION.

PHYS. LETT.,

V.149B, P.288-91. 1984

STROTTMAN, DANIEL D. (T-9)

GIBBS, WILLIAM R. (T-5)

$50-58$

HIGH RESOLUTION STUDIES OF PION NUCLEUS REACTION MECHANISM.

TIC,

1983. 15P. MN (LA-UR-83-1746)

MORRIS, CHRISTOPHER L. (MP-10)
50-59

HIGH SPIN STATES IN 28 SILICON AND

58 NICKEL

PHYS. REV.,

V.30C, P.1976-87. 1984

HINTZ, N.M.

FRANEY, M. A.

GAZZALY, MAGDI

COOK, DENNIS R.

KYLE, GARYS.

DEHNHARD, DIETRICH

SEESTROM-MORRIS, SUSAN J.

HOFFMANN, G. W. (MP-10)

BARLETT, $M$.

BLANPIED, GARY S. (MP-10)

MC GILL, JOHN A.

MC CLELLAND, JOHN B. (MP-10)

BOUDRIE, RICHARD L. (MP-10)

IROM, FAROKH

PAULETTA, G.

50-60

HYDRODYNAMICS AND RELATIVISTIC HEAVY

ION COLLISIONS.

TIC,

1983. 29P. MN (LA-UR-83-1839)

STROTTMAN, DANIEL D. (T-9)

50-61

IN THE MATTER OF THE H-BOMB.

BULL. AT. SCI.,

V.40, NO.2, P.52-3. 1984

MANLEY, JOHN $H$.

50-62

INCLUSIVE SCATTERING OF 5OO-MEV

PROTONS AND PIONIC ENHANCEMENT OF THE

NUCLEAR SEA-QUARK DISTRIBUTION

PHYS. REV. LETT.

V.53, P.144-7. 1984.

CAREY, THOMAS A. (P-3)

JONES, K. W. (MP-10)

MC CLELLAND, JOHN B. (MP-10)

MOSS, JOEL MARSHALL (P-2)

REES, L. (P-2)

TANAKA, NOBUYUKI (MP-10)

BACHER, ANDY D. (P-7)

50-63

INDEPENDENT YIELDS OF THE ISOMERS OF

133 XENON AND 135 XENON FOR NEUTRON

INDUCED FISSION OF 233 URANIUM,

235 URANIUM, 238 URANIUM, 239 PLUTONIUM, AND 242 AMERICIUM.

PHYS. REV.

V.30C, P.195-208. 1984.

FORD, GEORGE P. (CNC-11)

WOLFSBERG, KURT (INC-7)

ERDAL, BRUCE R. (INC-DO)
50-64

INELASTIC NEUTRON SCATTERING FROM

AMORPHOUS HYDRIDE OF

ZIRCONIUM 2 PALLADIUM

TIC,

1983. 7P. MN (LA-UR-83-2215)

WILLIAMS, ARTHUR (P-8)

ECKERT, JUERGEN (P-B)

YEH, XIAN L

ATZMON, MICHAEL

SAMWER, KONRAD

50-65

INTERFERENCE EFFECTS IN (PI, PI N)

REACTIONS.

PHYS. REV.

V.30C, P.254-63. 1984

OHKUBO, YOSHI (CNC-11)

LIU, LON CHANG (INC-11)

$50-66$

ISOSPIN DEPENDENCE OF PION ABSORPTION

ON NUCLEON PAIRS.

PHYS. REV.,

V.29C, P.1116-8. 1984.

SILBAR, RICHARD R. (T-5)

PIASETZKY,ELI

50-67

ISOVECTOR SPIN STATES OBSERVED IN

RADIATIVE PION CAPTURE IN FLIGHT AND

AT REST - RECENT RESULTS FROM SIN.

INTL CONF. ON SPIN EXCITATIONS

IN NUCLEI, 1982, TELLURIDE, PROC.,

P.559-77. PLENUM, 1984.

(539.7/161SP/1982)

LEBRUN, $M$.

MARTOFF, C.J.

STRAUMANN, $U$.

TRUOL, P.

JOSEPH, C.

PERROUD, J.P.

TRAN, M.T.

BISTIRLICH, J.A.

CROWE, KENNETH $M$.

DEUTSCH, J.

GREGOIRE GHISLAIN

PRIEELS, R.

DAHME, W.

BAER, HELMUT (MP-4)

$50-68$

K POSITIVE NUCLEUS ELASTIC SCATTERING AND CHARGE EXCHANGE.

PHYS. REV.

V.30C, P.1256-65. 1984

SIEGEL, PETER B.

KAUFMANN, WILLIAM B. (T-5)

GIBBS, WILLIAM R. (T-5) 
$50-69$

KINEMATICALLY COMPLETE MEASUREMENT OF THE (POSITIVE PION NEGATIVE PION, POSITIVE PION NEGATIVE PION PROTON) REACTION ON CARBON 12 AT $220 \mathrm{MEV}$.

PHYS. REV.,

V.30C, P.1622-30. 1984

FAUCET, JOHN A

WOOD, B. E. (P-15)

MCDANIELS, DAVDK

GRAM, PETER A. M. (MP-4)

HAMM, MARIANNE E. (Q-2)

OOTHOUDT, M. A. (MP-1)

GOULDING, CHARLES A. (Q-2)

SWENSON, L. W.

KRANE, KENNETH S.

STETZ, A. W.

PLENDL, H. S.

NORTON, $J$.

FUNSTEN, $\mathrm{H}$ JOYCE, $D$.

$50-70$

KINEMATICALLY COMPLETE MEASUREMENT OF PROTON KNOCKOUT BY 220 MEV PIONS ON CARBON 12.

LOS ALAMOS,

1984. 150P, (LA-09970-T)

FAUCETT, JOHN A

$50-71$

LASER-RAMAN REMOTE TEMPERATURE

SENSING IN UQUIDS.

EXP. FLUIDS,

V.2, P.81-8. 1984

PAN, PAUL Y. (Q-6)

FAW, R. E.

LESTER, T. W.

$50-72$

UNESHAPE ANALYSIS AND FILTER DIFFERENCE METHOD FOR A HIGH INTENSITY TIME - OF - FUGHT INELASTIC NEUTRON SCATTERING SPECTROMETER.

NUCL. INSTRUM. METHODS,

V.221, P.408-18. 1984.

TAYLOR, ANDREW D. (P-8)

WOOD, ELIZABETH J. (P-8)

GOLDSTONE, JOYCE A. (P-8)

ECKERT, JUERGEN (P-8)

$50-73$

LOW - Q INELASTIC SCATTERING WITH

EV NEUTRONS.

NUCL. INSTRUM. METHODS PHYS. RES.,

V.224, P.133-41. 1984

TAYLOR, ANDREW D. (P-8)

ROBINSON, ROBERT A. (P-8)

SEEGER, PHILIP A. (P-8)
$50-74$

MEASUREMENT AND ANALYSIS OF MUONIC

X RAYS OF 176, 177, 178, 179, 180

HAFNIUM.

PHYS. REV.,

V.30C, P.350-9. 1984

TANAKA, YASUTOSHI (P-7)

STEFFEN, ROLF MARCEL

SHERA, E. BROOKS (P-3)

REUTER, WILFRIED (P-3)

HOEHN, MARTHA V. (MP-13)

ZUMBRO, J. D.

50-75

MEASUREMENT AND ANALYSIS OF THE

MUONIC X-RAYS OF 151 EUROPIUM AND

153 EUROPIUM.

PHYS. REV.,

V.29C, P.1897-1904. 1984.

TANAKA, YASUTOSHI (P-7)

STEFFEN, ROLF MARCEL

SHERA, E. BROOKS (P-3)

REUTER, WILFRIED (P-3)

HOEHN, MARTHA V. (MP-13)

ZUMBAO. J. D.

$50-76$

MEASUREMENT OF THE WOLFENSTEIN

PARAMETERS FOR PROTON - PROTON AND

PROTON - NEUTRON SCATTERING AT

$500 \mathrm{MEV}$.

LOS ALAMOS,

1984. 120P. (LA-10171-T)

MARSHALL, J.A.

$50-77$

MEASUREMENT OF 14 - MEV NEUTRON CROSS

SECTIONS FOR ZIRCONIUM 88 AND

YTTRIUM 88.

PHYS. REV,

V.29C, P.805-10. 1984.

PRESTWOOD, RENE J. (INC-11)

THOMAS, KIMBERLY W (INC-11)

NETHAWAY, DAVD R

SMITH, NORMAN L.

$50-78$

MEDIUM ENERGY PROBES AND NUCLEAR

STRUCTURE.

NUCL. PHYS.

VA421, P.369G-86C. 1984.

GINOCCHIO, JOSEPHN. (T-5)

$50-79$

MODEL DEPENDENCE OF PERIPHERAL NN

PHASE PARAMETERS AT MEDIUM ENERGY.

PHYS. REV.

V.30C,P.1653-61. 1984

KLOET, WILLEM M.

TJON, JOHN (T-5)
$50-80$

NATURE OF THE COUPLING IN SUBTHAESHOLD

FISSION OF 238 NEPTUNIUM.

PHYS. REV.

V.29C, P.174-80. 1984.

AUCHAMPAUGH, GEORGE F. (P-3)

MOORE, MICHAEL S. (P-3)

MOSES, JOHN D. (P-3)

NELSON, RONALD O. (P-9)

EXTERMANN, RICHARD C. (P-3)

OLSEN, CLAYTON E. (MST-13)

HIL, N. W.

HARVEY, JEFFREY A

$50-81$

NEED FOR AN INTENSE POLARIZED SOURCE

AT LAMPF. REPORT ON A WORKSHOP HELD AT LOS ALAMOS, NOVEMBER 8, 1983.

LOS ALAMOS

1984. 28P. (LA-10051-MS)

MC NAUGHTON, MICHAEL W. (MP-13)

SILBAR, RICHARD R. (T-5)

VAN DYCK, OLN B. (MP-13)

$50-82$

NEUTRON OSCILLATION EXPERIMENTS USING ULTRACOLD NEUTRONS.

LOW ENERGY TESTS OF CONSERVATION

LAWS IN PARTICLE PHYSICS, 1983

BLACKSBURG. PROC., P.47-53. AJP

1984. (539.72/L912EN/1983)

ALSO PUBUSHED IN:

TIC,

1984. 14P. MN (LA-UR-83-2648)

PETSCHEK, ALBERT G. (P-DO)

$50-83$

NEUTRON SPECTRUM FROM AN IRON-

ALUMINUM - SULFUR FILTER.

TRANS. AM. NUCL. SOC.

V.47, P.388-90. 1984.

EVANS, ALBERT E., JR. (Q-2)

BENDT, PHILIP J. (P-2)

50-84

NEW EVALUATION OF FISSION-FRAGMENT

ANGULAR DISTRIBUTIONS IN HEAY - ION

REACTIONS.

PHYS. REV. LETT.

V.52, P.589-92. 1984.

GAVRON AVIGOR I. (P-3)

ESKOLA, PIRKKO L. (INC-11)

SIERK, ARNOLD J. (T-9)

BOISSEVAIN, JAN G. (P-3)

BRITT, HAROLD C. (P-3)

ESKOLA, KARI A. (INC-11)

FOWLR, MALCOLM M. (INC-7)

OHM, HENNER (P-3)

WILHELMY, JERRY B. (INC-11)

WALD, $\mathbf{S}$.

FERGUSON, R. L. 
50-85

NONMESONIC DECAY OF HEAYY LAMBDA HYPERNUCLEI.

PHYS. REV.,

V.30C, P.322-30. 1984.

MC KELUAR, BRUCE H. J. (T-5)

GIBSON, BENJAMIN F. (T-5)

60-86

NUCLEAR CHARGE DENSITIES IN THE

TRANSITION REGION 192 OSMIUM.

PHYS. LETT.

V.137B, P.32-6. 1984.

AEUTER, WILFRIED (P-3)

SHERA, E. BROOKS (P-3)

HOEHN, MARTHA Y. (MP-13)

HERSMAN, F. W.

MILLIMAN, T.

FINN, J.

HYDE-WRIGHT, C.

LOURIE, $R$

PUGH, B.

BERTOZZI, $W$.

50-87

NUCLEAR EFFECTS ON ION HEATING WITHIN

THE SMALL-ANGLE CHARGED PARTICLE

ELASTIC-SCATTERING REGIME.

PHYS. REV.

V.30A, P.1940-7. 1984.

ANDRADE, ANTHONY (X-2)

HALE, GERALD M. (T-2)

$60-88$

NUCLEAR INERTIA FOR FISSION IN A

GENERALIZED CRANKING MODEL

TIC,

1984. 6P. MN (LA-UR-84-0761)

KUNZ, JUTTA (T-9)

NIX, JAMES R. (T-9)

60-89

NUCLEAR STRUCTURE EFFECTS IN PION

SINGLE CHARGE EXCHANGE

PHYS. REV.

V.29C, P.526-37. 1984.

AUERBACH, NAFTALI

JOHNSON, MIKKEL B. (MP-DO)

KLEIN, AMIR

SICILIANO, EDWARD R.

50-90

ONE-GLUON-EXCHANGE AND THE SHORT-

RANGE PART OF THE PION - NUCLEON AND

PION - PION INTERACTION.

PHYS. LETT.,

V.144B, P.163-7. 1984.

HOLINDE, KARL (MP-DO)

JOHNSON, MIKKEL B. (MP-DO)

50-91

ONE-PION-EXCHANGE POTENTIAL DEUTERON.

PHYS. REV.

V.30C, P.1084-6. 1984.

FRIAR, JAMES L. (T-5)

GIBSON, BENJAMIN F. (T-5)

PAYNE, GERALD L.
$50-92$

PARITY NONCONSERVATION IN A PROTON

NUCLEUS SCATTEAING AT 6 GEV/C.

PHYS. REV.,

V.30D, P.860-70. 1984.

LOCKYER, $N$.

ROMANOWSKI, T. A

BOWMAN, J. DAVID (MP-4)

HOFFMAN, CYRUS M. (MP-4)

MISCHKE, RICHARD E. (MP-4)

NAGLE, DARRAGHE. (MP-DO)

POTTER, J. M. (AT-1)

TALAGA, R. L.

SWALLOW, E.C.

ALDE, D. M.

MOFFETT, D. R.

ZYSKIND, JOHN

$50-93$

PION - INDUCED PION PRODUCTION ON THE DEUTERON.

PHYS. REV. LETT.,

V.53, P.540-3. 1984

PIASETZKY, ELI

GRAM, PETER A. M. (MP-4)

MAC ARTHUR, DUNCAN W.

REBKA, G. A

BORDNER, C. A

HOIBRATEN, $\mathbf{S}$.

KINNEY, E. R.

MATTHEWS, J.L.

WOOD, S. A.

ASHERY, D.

LICHTENSTADT, $J$.

$50-94$

PION - NUCLEUS ELASTIC SCATTERING AT

80 MEV.

PHYS. REV.,

V.29C, P.561-8. 1984.

LEITCH, M. J. (MP-4)

BURMAN, ROBERT L. (MP-4)

CARLINI, ROGER D. (MP-4)

DAM, STEVEN H.

SANDBERG, V. D. (MP-4)

BLECHER, $M$

GOTOW, K

NG, $R$.

AUBLE, $R$.

BERTRAND, F.E.

GROSS, E. E.

OBENSHAIN, F.E.

WU, J.

BLANPIED, GARY S. (MP-10)

PREEDOM, BARRY $M$.

RITCHIE, B. C.

BEATOZZI, W

HYNES, M. V. (P-15)

KOVASH, M. A

REDWINE, ROBERT P. (MP-4)

$50-95$

PION CHARGE EXCHANGE AND NUCLEAR

STRUCTURE

COMMENTS NUCL. PART. PHYS.,

V.12,P.299-309. 1984.

BOWMAN, J. DAVID (MP-4)

COOPER, MARTIN D. (MP-4)
50-96

PION CHARGE EXCHANGE FROM ORIENTED,

DEFORMED NUCLEI.

PHYS. REV. LET.,

V.53, P.1996-9. 1984.

CHIANG, H. C.

JOHNSON, MIKKEL B. (MP-DO)

60-97

PION DOUBLE CHARGE EXCHANGE ON

T EQUALS 1 NUCLEI.

PHYS. REV.,

V.30C, P.973-8. 1984.

SEIDL, PETER A.

BROWN, MARKK

KIZIAH, R. R.

MOORE, C. FRED

BAER, HELMUT (MP-4)

MORRIS, CHRISTOPHER L (MP-10)

BURLESON, GEORGE R.

COTTINGAME, W. B.

GREENE, S. J. (MP-10)

BLAND, L. C.

CILMAN, R.

FORTUNE, H.T.

$50-98$

PION ELASTIC AND INELASTIC SCATTERINC FROM SAMARIUM 152.

PHYS. REV.

V.28C, P.2165-7. 1883.

MORRIS, CHRISTOPHER L (MP-10)

SEESTROM-MORRIS, SUSAN J.

SEIDL, PETER A

KIZIAH, R. R.

GREENE, S. J.

50-99

PION ELASTIC AND INELASTIC SCATTERING FROM 40, 42, 44, 48 CALCIUM AND

54 IRON.

PHYS. REV.

V.29C, P.182-93. 1984.

BOYER, K. G. (MP-10)

BRATTHWAITE, WILFRED J.

COTTINGAME, $W . B$.

GREENE, S. J.

SMITH, L. E. (MP-10)

MOORE, C. FRED

MORRIS, CHRISTOPHER L. (MP-10)

THIESSEN, HENAY A. (MP-10)

BLANPIED, GARY S. (MP-10)

BURLESON, GEORGE R.

DAVS, JOHN F., III (a-14)

MC CARTHY, J.S.

MINEHART, R. C.

GOULDING, CHARLES A.

50-100

PION ELASTIC AND INELASTIC SCATTERING

FROM 40, 42, 44, 48 CALCIUM AND

54 IRON.

LOS ALAMOS,

1984. 391P. (LA-09974-T)

BOYER, K. G. (MP-10) 
50-101

PION-NUCLEUS ABSORPTION VIA

THE DELTA - NUCLEON INTERMEDIATE STATE.

PHYS. REV.,

V.30C, P.720-2. 1984.

FRAENKEL, ZEEV (P-3)

PIASETZKY, ELI

CLOVER, M. R. (P-2)

50-102

PIONIC $M$ X-RAYS OF 166,168 ERBIUM AND

176 HAFNIUM.

PHYS. LETT.

V.143B, P.347-50. 1984.

TANAKA, YASUTOSHI (P-7)

STEFFEN, ROLF MARCEL

SHERA, E. BROOKS (P-3)

REUTER, WILFRIED (P-3)

HOEHN, MARTHA V. (MP-13)

ZUMBRO, J.D.

50-103

PLASMA ENERGY DEPOSITION FROM THE NUCLEAR ELASTIC SCATTERING OF TRITONS ON TRITONS.

NUCL. SCI. ENG.

V.88, P.592-3. 1984

DEVANEY, JOSEPH J. (X-6)

50-104

POLARIZATION PHENOMENA IN N - NUCLEUS SCATTERING.

TIC,

1983. 17P. MN (LA-UR-83-2531)

MOSS, JOEL MARSHALL (P-3)

50-105

PRODUCTION OF NEUTRON-RICH BISMUTH

ISOTOPES BY TRANSFER REACTIONS.

PHYS. REV

V.29C, P.2160-7. 1984

ESKOLA, KARI A. (INC-11)

ESKOLA, PIRKKO L. (INC-11)

FOWLER, MALCOLM M. (INC-7)

OHM, HENNER (P-3)

TREHER, ELIZABETH N. (INC-7)

WILHELMY, JERRY B. (INC-11)

LEE, DAVID

SEABORG, GLENN T.

50-106

PROMPT FISSION NEUTRON SPECTRA AND

AVERAGE PROMPT NEUTRON MULTIPLICITIES.

TIC

1983. 36P. MN (LA-UR-83-3074)

MADLAND, DAVID G. (T-2)

NIX, JAMES R. (T-9)
50-107

PROPERTIES OF THE ISOVECTOR MONOPOLE

AND OTHER GIANT RESONANCES IN PION

CHARGE EXCHANGE.

PHYS. REV. LETT.

V.52, P.2134-7. 1984.

ERELL, A.

ALSTER, JONAS

LICHTENSTADT, J.

MOINESTER, MURRAY A

BOWMAN, J. DAVID (MP-4)

COOPER, MARTIN D. (MP-4)

IROM, FAROKH

MATIS, H. S. (MP-4)

PIASETZKY, EL

SENNHAUSER, URS (MP-4)

INGRAM, $\mathbf{Q}$.

50-108

QPAN - A QUICK POLARIZATION ANALYZER

FOR NEUTRONS.

NUCL. INSTRUM. METHODS,

V.219, P.275-8. 1984

HOLLAS, C. L.

RANSOME, R. D.

RILEY,P. J.

BONNER, BILLY E. (P-3)

BOISSEVAIN, JAN G. (P-3)

BHATIA, TAROLCHAN S.

GLASS, G.

HIEBERT, J. C.

TIPPENS, $\boldsymbol{W} . \mathbf{B}$.

50-109

QUARK TUNNELING IN HYPERNUCLEI.

TIC,

1984. 8P. MN (LA-UR-84-1873)

GOLDMAN, TERRENCE J. (T-5)

50-110

QUARK TUNNELING IN NUCLEI.

PHYS. LETT.

V.146B, P.143-7. 1984.

GOLDMAN, TERRENCE J. (T-5)

STEPHENSON, GERARD J., JR. (T-5)

50-111

RADIATION DAMAGE CALCULATIONS WITH

NJOY.

TIC.

1983. 7P. MN (LA-UR-83-2566)

MAC FARLANE, ROBERT E. (T-2)

MUIR, DOUGLAS W. (T-2)

MANN, FREDERICK $W$.

50-112

RECENT DEVELOPMENTS IN THE UNDERSTANDING

OF PION - NUCLEUS SCATTERING.

TIC

1983. 55P. MN (LA-UR-83-2851) JOHNSON, MIKKEL B. (MP-DO)
$50-113$

RECOIL MEASUREMENT OF THE CARBON 12 (POSITIVE PION NEGATIVE PION,

POSITIVE PION NEGATIVE PION NEUTRON) CARBON 11 REACTION BETWEEN 90 AND 350 MEV.

PHYS. REV.

V.29C, P.2239-42. 1984

PORILE, NORBERT T.

CARETTO, ALBERT A.

DROPESKY, BRUCE J. (INC-11)

ORTH, CHARLES J. (INC-11)

LIU, LON CHANG (INC-11)

GIESLER, GREGG CARL (INC-11)

50-114

RELATIVISTICALLY CORRECTED SCHRODINGER

EQUATION WITH COULOMB INTERACTION.

PHYS. REV.

V.29C, P.1537-9. 1984.

FRIAR, JAMES L. (T-5)

TOMUSIAK, EDWARD L.

50-115

REPLY TO "COMMENT ON (PROTON, NEUTRON)

AND (NEUTRON, PROTON) REACTIONS AS

PROBES OF ISOVECTOR GIANT MONOPOLE

RESONANCES."

PHYS. REV

V.30C, P.736-7. 1984.

AUERBACH, NAFTALI

BOWMAN, J. DAVID (MP-4)

FRANEY, M. A.

LOVE, W. GARY

$50-116$

SMALL ANGLE PION INELASIC SCATTERING FROM 12 CARBON AT 162 MEV.

PHYS. REV.

V.30C, P.662-5. 1984.

MORRIS, CHRISTOPHER L. (MP-10)

TANAKA, NOBUYUKI (MP-10)

BOUDRIE, RICHARD L. (MP-10)

BLAND, L. C.

FORTUNE, H. T.

GILMAN, $R$.

SEESTROM-MORRIS, SUSAN J.

MOORE, C. FRED

DEHNHARD, DIETRICH

50-117

SPIN DEPENDENCE OF NN AND NN PI

REACTIONS AND THE QUESTION OF

DIBARYON RESONANCES.

COMMENTS NUCL. PART. PHYS.,

V.12, P.177-89. 1984.

SILBAR, RICHARD R. (T-5) 
50-118

SPIN EXCITATIONS IN PION INELASTIC

SCATTERING

INTL. CONF. ON SPIN EXCITATIONS

IN NUCLEI, 1982, TELLURIDE. PROC.,

P.291-309. PLENUM, 1984.

(539.7/161SP/1982)

ALSO PUBLISHED IN:

TIC.

1982. 2OP. MN (LA-UR-82-0854)

SEESTROM-MORRIS, SUSAN J.

HOLTKAMP, DAVID B. (MP-10)

COTTINGAME, W. B.

50-119

SPIN OBSERVABLES IN NUCLEON - NUCLEUS

SCATTERING

INTERNATIONAL CONFERENCE ON SPIN

EXCITATIONS IN NUCLEI, 1982.

TELLURIDE. PROC., P.355-68. PLENUM, 1984.

MOSS, JOEL MARSHALL (P-3)

50-120

SPONTANEOUS-FISSION PROPERTIES AND

PRODUCTION OF HEAW-ELEMENT ISOTOPES.

ACC. CHEM. RES.,

V.17, P.235-42. 1984.

HOFFMAN, DARLEANE C. (INC-DO)

50-121

STATEMENT OF DR. STEVE ROCKWOOD,

LOS ALAMOS NATIONAL LABORATORY.

FUSION ENERGY WORKSHOP,

JOINT HEARING BEFORE U.S. HOUSE AND

SENATE SUBCOMMITTEES, WASHINGTON, D.C

SEPT. 8, 1982. U.S.G.P.O., 1983.

(328/C97-2/82-85)

ROCKWOOD, STEPHEN D. (NSP/IF)

50-122

STATIONARY FOKKER - PLANCK EQUATION

APPLIED TO FISSION DYNAMICS.

NUCL. PHYS.

V.A424, P.239-60. 1984

NIX, JAMES R. (T-9)

SIERK, ARNOLD J. (T-9)

HOFMANN, HELMUT

SCHEUTER, FRANZ J.

VAUTHERIN, DOMINIQUE

$50-123$

STATUS OF FISSION YIELD EVALUATIONS.

TiC.

1983. 33P. MN (LA-UR-83-3531)

ENGLAND, TALMADGE R. (T-2)

RIDEA, B. F.

$50-124$

STATUS REPORT ON THE WNR/PSR PULSED

SPALLATION NEUTRON SOURCE AT THE LOS

ALAMOS NATIONAL LABORATORY.

TIC,

1983. 7P. MN (LA-UR-83-3200)

BOWMAN, CHARLES D. (P-DO)
50-125

STRUCTURE OF ISOVECTOR SPIN EXCITATIONS

IN NUCLEI.

PHYS. REV.,

V.30C, P.1032-42. 1984.

AUERBACH, NAFTALI

KLEIN, AMIF

50-126

STUDY OF GIANT RESONANCES WITH PIONS

TIC.

1984. 27P. MN (LA-UR-84-1293)

BAER, H. W. (MP-4)

50-127

THE KAON PLUS NUCLEUS INTERACTION.

TIC.

1984. 9P. MN (LA-UR-84-1871)

GIBBS, WILLIAM R. (T-5)

$50-128$

THEORETICAL ASPECTS OF DOUBLE BETA

DECAY.

TIC.

1984. 17P. MN (LA-UR-84-1623) HAXTON, WICK C. (T-5)

$50-129$

THERMAL EQUILIBAIUM POPULATION OF THE FIRST FEW NUCLEAR EXCITED STATES (ACTINIUM, THORIUM, PROTACTINIUM, URANIUM, NEPTUNIUM, PLUTONIUM, AMERICIUM, CURIUM, BERKELIUM, AND CALIFORNIUM ISOTOPES).

LOS ALAMOS,

1984. 83P. (LA-10111-MS)

DOOLEN, GARY D. $(X-5)$

HSU, H. H. (X-5)

DOOLEN, C. L

50-130

THERMALIYINDUCED OPTICAL SCINTILLATIONS FROM SINGLE CRYSTALS OF UNIRRADIATED BERYLLIUM OXIDE - LITHIUM AND

BERYLLIUM OXIDE - SODIUM.

FERROELECTRICS,

V.59,P.187-95. 1984

COOKE, D. W. (MP-3)

LIANOS, D.

GAVATHAS, E.

ALEXANDER, $C$.

SHUL'GIN, B. V.

JAHAN, M. SHAH

50-131

THICK OSMIUM TARGETS

NUCL. INSTRUM. METHODS,

V.220, P.288-92. 1984.

REUTER, WILFRIED (P-3)

GURSKY, JUDITH C. (P-3)

SHERA, E. BROOKS (P-3)

JOHNSON, M. W. (AT-5)
50-132

TIME REVERSAL INVARIANCE IN POLARIZED NEUTRON BETA DECAY

TIC,

1983. 5P. MN (LA-UR-83-3250)

BOWLES, THOMAS J. (P-3)

MOSES, JOHN D. (P-3)

SUNIER, JULES W. (P-3)

BROWN, RONALD E. (P-3)

LISOWSKI, PAUL W. (P-3)

MAMPE, $W$.

LIAUD, $P$.

50-133

TPC SPECTROMETER FOR MEASURING THE

E PLUS SPECTRUM IN MICRON DECAY.

TIME PROJECTION CHAMBER, 1983,

VANCOUVER. PROC., P.21-41. AMER.

INSTITUTE OF PHYSICS, 1984.

(539.77/T583PR/1983)

ALSO PUBLISHED IN:

TIC,

1983. 17P. MN (LA-UR-83-2485)

KINNISON, W. WAYNE (MP-4)

50-134

TRACK RECONSTRUCTION OF NORMAL MUON DECAYS IN THE LAMPF TPC - ONE WORKING

SCHEME.

TIME PROJECTION CHAMBER, 1983,

VANCOUVER. PROC., P.242-61. AMER.

INSTITUTE OF PHYSICS, 1984.

(539.77/T583PR/1983)

ALSO PUBLISHED IN:

TIC

1983. 21P. MN (LA-UR-83-2839)

MC KEE, ROBERT J. (P-DO)

50-135

UPPER LIMIT ON THE ISOVECTOR PARITY

VIOLATING DECAY WDTH OF THE OXYGEN PLUS

T EQUALS 1 STATE OF LITHIUM 6.

PHYS. REV.,

V.29C, P.755-63. 1984.

ROBERTSON, ROBERT G. H. (P-3)

DYER, PEGGY (P-3)

MELIN, ROBERT C.

BOWLES, THOMAS J. (P-3)

MC DONALD, ARTHUR BRUCE

BALL, GORDON C.

DAVES, WALTER G.

EARLE, E. DAVIS

$50-136$

USE OF GASIMETRIC, TIME-TO-EXPLOSION

AND ISOTHERMAL DIFFERENTIAL SCANNING

CALORIMETRY TO ASSESS COMPATIBILITY OF

DOUBLE-BASE PROPELLANTS ANO EPOXY RESIN

JOINT SYMPOSIUM - COMPATIBILITY OF

PLASTICS/MATERIALS WITH EXPLOSIVES

PROPELLANTS, PYROTECHNICS

INGREDIENTS, 1983, LAKE OZARK. PROC.

18P. ADPA, 1983. (662.2/J74CO/1983)

CALDWELL, DENNIS J.

EDWARDS, JEFFERY B.

KEIFER, JOHN R.

ROGERS, RAYMONDN. (M-1) 
50-137

12 CARBON(NEUTRON,NEUTRON')B ALPHA

CROSS SECTION UP TO $60 \mathrm{MEV.}$

NUCL. SCI. ENG.

V.88, P.97-101. 1984.

BRENNER, DAVID J. (MP-3)

PRAEL, RICHARD E. (X-6)
50-138

3 HELIUM (PROTON, PROTON) 3 HELIUM

SCATTERING IN THE ENERGY RANGE 19 TO

48 MEV.

PHYS. REV.

V.29C, P.2001-7. 1984.

MURDOCH, BRUCE T.

HASELL, D. K.

SOURKES, A. M

VAN OERS, WILLM T. H.

VERHEWEN, P.J.T.

BROWN, RONALD E. (P-3) 


\section{PHYSICS-PARTICLES AND FIELDS}

51-1

A SULFUR OXIDE 8 MODEL OF COLLECTIVITY

IN NUCLEI.

WORKSHOP ON BOSONS IN NUCLEI, 1983,

DREXEL. PROC., P.81-105.

WORLD SCIENTIFIC, 1984

(539.72/N926BO/1983)

ALSO PUBLISHED IN:

TIC.

1983. 25P. MN (LA-UR-83-1310)

GINOCCHIO, JOSEPHN. (T-5)

61-2

ANALYTICITY AND THE BOREL SUMMABILTTY

OF ASYMPTOTICALLY FREE THEORIES.

PHYS. LETT.

V.145B, P.103-7. 1984.

WEST, GEOFFREY B. (T-8)

$51-3$

BOSON - MEDIATED INTERACTIONS BETWEEN

STATIC SOURCES.

QUARKS AND NUCLEAR STRUCTURE, 3RD,

1983, BAD HONNEF, PROC., P.405-14.

SPRINGER-VERLAG, 1983.

(539.72/K63ER/3RD/1983)

ALSO PUBLSHED IN:

TIC,

1983. 11P. MN (LA-UR-B3-2973) BOLSTERLI, MARK (T-9)

$51-4$

COMMENT ON THE LIGHT - HEAVY MANORANA NEUTRINO MECHANISM IN NO - NEUTRINO DOUBLE BETA DECAY.

TIC.

1984. 5P. MN (LA-UR-84-0825)

ROSEN, S. P. (T-6)

51-5

COMMENT ON THE ULTAAVIOLET BEHAVIOR OF GAUGE-DEPENDENT GREEN'S FUNCTIONS

WOCD.

PHYS. REV.

V.30D, P.1349-62. 1984.

WEST, GEOFFREY B. (T-B)

$51-6$

COORDINATES FOR AN OPTIMUM CS

APPROXIMATION IN REACTIVE SCATTERING.

CHEM. PHYS. LETT.

V.108, P.333-7. 1984

PACK, RUSSELL T. (T-12)

$61-7$

EXACTNESS OF MODERN QUANTUM-MECHANICAL

EFFECTS.

PHYS. REV.

V.29A, P.3413-5. 1984.

NIETO, MICHAEL MARTIN (T-8)
$51-8$

EXPERIMENTS TO TEST UNIFICATION

SCHEMES.

LOS ALAMOS SCI.

NO.11, SUMMER-FALL, P.124-44. 1984. SANDERS, GARY H (MP-13)

51-9

FAMILY PROBLEM.

LOS ALAMOS SCI.,

NO.11, SUMMER-FALL, P.115-23. 1984. GOLDMAN, TERRENCE J. (T-6) NIETO, MICHAEL MARTIN (T-8)

61-10

FUN WITH SUPERSYMMETAIC QUANTUM MECHANICS

TIC,

1984. 21P. MN (LA-UR-84-1311)

FREEDMAN, BARRYA.

COOPER, FREDERICK M. (T-8)

$51-11$

GENERAL CP PROPERTIES OF NEUTRINO MASS

EIGENSTATES.

PHYS. REV.,

V.29D, P.2535-8. 1984

ROSEN, S.P. (T-8)

51-12

GENERAL CP PROPERTIES OF NEUTRINO MASS EIGENSTATES.

TIC.

1984. 6P. MN (LA-UR-84-0824) ROSEN, S. P. (T-B)

51-13

HADRONIC MULTIPLICITY DISTRIBUTIONSEXAMPLE OF A UNIVERSAL STOCHASTIC PROCESS.

TIC.

1983. 1OP. MN (LA-UR-83-2041) CARRUTHERS, PETER A. (T-DO)

51-14

IMPOSSIBILITY OF NAIVELY GENERALIZING SQUEEZED COHERENT STATES.

PHYS. REV.,

V.29D, P.1107-10. 1984.

FISHER, ROBERT A. (CHM-6)

NIETO, MICHAEL MARTIN (T-8)

SANDBERG, V. D. (MP-4)
51-15

INFLUENCE OF SOLITONS IN THE INTTIAL STATE ON CHAOS IN THE DRIVEN DAMPED SINE-GORDON SYSTEM.

ORDER IN CHAOS, 1982, LOS ALAMOS. PROC. P.259-79. NORTH HOLLAND, 1983. (003.2/161OR/2ND/1982)

ALSO PUBLISHED IN:

PHYSICA,

V.7D, P.259-79. 1983.

BISHOP, ALAN R. (T-11)

FESSER, KLAUS (T-11)

LOMDAHL, PETER $S$.

TRULLINGER, STEVEN EDWARD

$51-16$

LAMPF II AND THE HIGH-INTENSITY

FRONTIER.

LOS ALAMOS SCI.

NO.11, SUMMER-FALL, P.152-7. 1984

THIESSEN, HENRY A. (MP-DO)

$51-17$

LECTURE NOTES FROM SIMPLE FIELD THEORIES TO THE STANDARD MODEL

LOS ALAMOS SCI.,

NO.11, SUMMER-FALL, P.54-71. 1984. SLANSKY, RICHARD C. (T-8)

51.18

LMITER OF CRITICAL EXPONENT

UNIVERSALTY

J.PHYS. A,

V.17, P.L621-6. 1984.

BAKER, GEORGE A., JR. (T-11)

51-19

MARCH TOWARD HIGHER ENERGIES.

LOS ALAMOS SCI.

NO.11, SUMMER-FALL, P.146-57. 1984 ROSEN, SIMON PETER (T-DO)

61-20

MESON FIELD QUENCHING OF PROTON DECAY.

PHYS. LETT.

V.142, P.47-61. 1984.

GOLDHABER, ALFRED S.

GOLDMAN, TERRENCE J. (T-5) NUSSINOV, S. (T-8)

51-21

NEUTRINO MASSES AND MIXINGS - AN

SPVAT ANALYSIS.

TIC,

1984. 16P. MN (LA-UR-84-0644)

GOLDMAN, TERRENCE J. (T-5) 
51-22

NO - NEUTRINO DOUBLE BETA DECAY - MORE THAN ONE NEUTRINO.

LOW ENERGY TESTS OF CONSERVATION

LAWS IN PARTICLE PHYSICS, 1983,

BLACKSBURG. PROC., P.176-83. AIP,

1984. (539.72/L912EN/1983)

ALSO PUBLISHED IN:

TIC,

1983. 8P. MN (LA-UR-83-3096)

ROSEN, S. P. (T-8)

$51-23$

ON THE DIMENSIONAL REDUCTION OF D

EQUALS 10, N EQUALS 2 SUPERGRAVITIES.

PHYS. LETT.

V.141B, P.187-90. 1984.

CHAPLINE, GEORGE

SLANSKY, RICHARD C. (T-8)

$51-24$

PARTICLE PHYSICS AND THE STANDARD

MODEL.

LOS ALAMOS SCI.

No.11, P.23-53. 1984.

RABY, STUART A. (T-8)

SLANSKY, RICHARD C. (T-8)

WEST, GEOFFREY B. (T-8)

51-25

PHYSICS AT THE PROPOSED NATIONAL UNDERGROUND SCIENCE FACILITY.

TIC,

1983. 15P. MN (LA-UR-83-2610)

NIETO, MICHAEL MARTIN (T-8)

51-26

CCD ON A CRAY - THE MASSES OF

ELEMENTARY PARTICLES.

LOS ALAMOS SCI.

NO.11, SUMMER-FALL, P.41. 1984.

GURALNIK, GERALD D. (T-DO)

WARNOCK, TONY T.

ZEMACH, CHARLES (T-DOT)

$51-27$

QUARKS \& QUIRKS AMONG FRIENDS.

LOS ALAMOS SCl.,

NO.11, SUMMER-FALL, P.172-87. 1984.

CARRUTHERS, PETER A. (T-8)

RABY, STUART A. (T-8)

SLANSKY, RICHARD C. $(T-8)$

WEST, GEOFFREY B. (T-8)

ZWEIG, GEORGE (T-DOT)

$51-28$

RELATIONSHIP BETWEEN SUPERSYMMETRY AND

THE INVERSE METHOD IN QUANTUM MECHANICS.

PHYS. LETT.,

V.145B, P.208-10. 1984.

NIETO, MICHAEL MARTIN (T-8)
51-29

RENORMALIZING THE EFFECTIVE POTENTIAL FOR SPONTANEOUSLY BROKEN G THETA 4 FIELD THEORY.

NUCL. PHYS.,

V.8239, P.459-76. 1984.

COOPER, FREDERICK M. (T-8)

FREEDMAN, BARRY A.

$51-30$

RESOLUTION OF THE IDENTITY FOR

MINIMUM-UNCERTAINTY COHERENT STATES

AN EXAMPLE RELATED TO CHARGED-BOSON

COHERENT STATES.

PHYS. REV.,

V.300, P.770-2. 1984.

NIETO, MICHAEL MARTIN (T-8)

$51-31$

SCALE AND DIMENSION - FROM ANIMALS

TO QUARKS.

LOS ALAMOS SCI.

NO.11, SUMMER-FALL, P.4-19. 1984. WEST, GEOFFREY B. $(T-8)$

$51-32$

SCIENCE UNDERGROUND - THE SEARCH

FOR RARE EVENTS.

LOS ALAMOS SCI.

NO.11, SUMMER-FALL, P.160-70. 1984. SIMMONS, LEONARD M., JR. (T-DO)

$51-33$

SEARCH FOR RARE DECAY OF THE MUON

USING THE CRYSTAL BOX DETECTOR. INTERNATIONAL WORKSHOP ON WEAK INTERACTIONS AND NEUTRINOS, 8TH, 1982, JAVEA. PROC., P.288-92.

WORLD SCIENTIFIC, 1983.

(539.72/161WE/8TH/1982)

NAGLE, DARRAGH E. (MP-DO)
51-34

SEARCH FOR THE MUON-NUMBER-NONCONSERVING DECAY MU GOING TO POSITIVE PION POSITIVE

PION NEGATIVE PION.

PHYS. REV. LETT.

V.53, P.1415-8. 1984. BOLTON, RICHARD D. (MP-4) BOWMAN, J. DAVD (MP-4)

CARLINI, ROGER D. (MP-4)

COOPER, MARTIN D. (MP-4)

DUONG-VAN, MINH

FRANK, JAMES S. (MP-4)

HALUN, AKSEL L (MP-4)

HEUSI, $P$.

HOFFMAN, CYRUS M. (MP-4)

MARIAM, FRANK (MP-13)

MATIS, H. S. (MP-4)

MISCHKE, RICHARD E. (MP-4)

NAGLE, DARRAGH E. (MP-DO)

SANDBERG, V. D. (MP-4)

SANDERS, GARY H (MP-13)

SENNHAUSER, URS (MP-4)

TALAGA, R. L.

WERBECK, RICHARD D. (MP-7)

WILLIAMS, ROBERT A. (MP-4)

WILSON, S. L.

HUGHES, E. BARRIE

HOFSTADTER, $R$.

GROSNICK, D.

WRIGHT, S. C.

HOGAN, GARY E. (MP-13)

HIGHLAND, V.L.

$51-35$

SOLTON DYNAMICS ON AN EASY-PLANE

FERROMAGNETIC CHAIN.

J.PHYS. C,

V.17,P.5975-90. 1984.

WYSIN, GARY

BISHOP, ALAN R. (T-11)

KUMAR, PRADEEP

51-36

SOLITON ENERGETICS IN PEIERLS-HUBBARD

MODELS.

PHYS. REV. LETT.

V.52, P.1717-20. 1984.

CAMPBELL, DAVIO K. (CNLS)

DE GRAND, $T$.

MAZUMDAR, SUMIT (CNLS)

$51-37$

SOLTTONS AND POLARONS IN QUASI - ONE

DIMENSIONAL CONDUCTING POLYMERS AND RELATED MATERIALS.

TIC.

1983. 33P. MN (LA-UR-83-2039)

CAMPBELL, DAVID K. (CNLS)

$51-38$

SPONTANEOUS DIMENSIONAL REDUCTION IN KALUZA - KLEIN THEORIES.

PHYS. REV.,

V.30D, P.339-42. 1984

TOSA, YASUNARI (T-8) 
$51-39$

SSC - AN ENGINEERING CHALLENGE

LOS ALAMOS SCI.,

NO.11, SUMMER-FALL, P.158-9. 1984

WILSON, MAHLON T. (AT-DO)

$51-40$

STATUS OF MU DECAYS.

TIC,

1983. 24P. MN (LA-UR-83-3359)

KINNISON, W. WAYNE (P-DO)

$51-41$

STRONG - COUPLING APPROXIMATION IN SUPERSYMMETRIC FIELD THEORY

PHYS. LETT.

V.134B, P.225-9. 1984.

BENDER, CARL $M$.

DAS, ASHOK

LIM, HWA-AUN

SIMMONS, LEONARD M., JR. (T-DO)

$51-42$

STUDY OF MUON-NUMBER-VIOLATING HYPERON DECAYS.

PHYS. REV.

V.29D, P.475-86. 1984

HERCZEG, PETER (T-5)

OKA, TAKAMITSU (T-5)
$51-43$

STUDY OF'PI ZERO GOING TO MU PLUS MINUS

ELECTRON MINUS PLUS DECAYS.

PHYS. REV.

V.29D, P.1954-61. 1984

HERCZEG, PETER (T-5)

HOFFMAN, CYRUS M. (MP-4)

$51-44$

SUPERSYMMETRY AT 100 GEV.

LOS ALAMOS SCI.

NO.11, SUMMER-FALL, P.98-112. 1985.

RABY, STUART A. (T-B)

$51-45$

TENSOR POLARIZATION IN PION-

DEUTERON SCATTERING AND PION

ABSORPTION.

PHYS. REV. LETT.,

V.52, P.333-6. 1984

UNGRICHT, E.

FREEMAN, W. S.

GEESAMAN, D. F.

HOLT, ROY J.

SPECHT, J. R.

ZEIDMAN, $B$.

STEPHENSON, EDWARD J.

MOSES, JOHN D. (P-3)

FARKHONDEH, $M$.

GILAD, S. (MP-4)

REDWINE, ROBERT P. (MP-4)

$51-46$

TOWARD A UNIFIED THEORY.

LOS ALAMOS SCI.

NO.11, SUMMER-FALL, P.74-95. 1984.

SLANSKY, RICHARD C. (T-8)
51.47

TRANSPORT THEORY (RADIATIVE TRANSFER). ENCYCLOPEDIA OF PHYSICS, ROBERT M. BESANCON, ED., P.1254-8. VAN NOSTRAND REINHOLD CO., 1985.

(530.032/E56PH/1985)

WING, G. MILTON (C-3) NELSON, PAUL

$51-48$

TWO - LOOP FINITE SUPERSYMMETRIC

SULFUR URANIUM 5 THEORY. TOWARDS A

THEORY OF FERMION MASSES.

PHYS. LETT.

V.143B, P.137-41. 1984

JONES, D. R. TIMOTHY

FABY, STUART A. (T-8)

$51-49$

WHY THE HADRONIC MULTIPLICTYY

DISTRIBUTIONS IN ETA POSITIVE ETA

NEGATIVE ANNIHILATIONS ARE SO NARROW.

PHYS. LETT.,

V.137B, P.425-30. 1984.

CARRUTHERS, PETER A. (T-DO)

SHIH, CHIA CHANG

$51-50$

XIX RECONTRE DE MORIOND - SUMMARY TALK. TIC,

1984. 23P. MN (LA-UR-84-0826)

ROSEN, S.P. (T-8) 
52. RADIOISOTOPE AND RADIATION APPLICATIONS

52-1

BEAM TRANSFER AND EXTRACTION AT LAMPF II.

TIC,

1983. 4P. MN (LA-UR-63-2456) COLTON, EUGENE P. (MP-14)
52-2

BENEFICIAL USES OF 241 AMERICIUM.

LOS ALAMOS.

1984. 27P. (LA-10075-MS)

MANGENG, GAROLYN A. (S-4)

THAYER, GARY R. (S-4) 


\section{SAFEGUARDS-NUCLEAR MATERIALS SECURITY}

53-1

AN END - CROP BOX COUNTER.

TIC,

1983. BP. MN (LA-UR-83-3199)

SPRINKLE, JAMES L, JR. (Q-1)

53-2

ANALYSIS OF TANK CALIBRATION DATA

FROM SEVERAL RUNS.

INSTITUTE OF NUCLEAR MATERIALS

MANAGEMENT. ANNUAL MEETING, 25TH,

1984, COLUMBUS. PROC.

IN - NUCLEAR MATERIALS MANAGEMENT,

V.13, P.149-53. 1984

GOLDMAN, AARON S. (Q-4)

LIEBETRAU, $A$.

53-3

APPLICATION OF THERMAL NEUTRON CAPTURE

GAMMA RAYS TO MARIX IDENTIFICATION INA

TRANSURANIC WASTEASSAY SYSTEM BASED ON

AN ELECTRON ACCELERATOR.

NUCL INSTRUM. METHODS,

V.220, P.531-6. 1984.

CLOSE, DONALD A. (Q-2)

FRANKS, L. A.

KOCIMSKI, S. M.

53-4

ARE 0.1 PERCENT - ACCURATE GAMMA - RAY

ASSAYS POSSIBLE FOR 235 URANIUM

SOLUTIONS.

TIC.

1983. 14P. MN (LA-UR-63-3265)

PARKER, JACKL (Q-1)

53-5

ARRAY OF GERMANIUM DETECTORS FOR

NUCLEAR SAFEGUARDS.

TIC,

1983. 5P. MN (LA-UR-83-1932)

MOSS, CALVINE. (Q-2)

BERNARD, WILLLAM (Q-2)

DOWDY, EDWARD J. (Q-2)

GARCIA CLEMENTE (Q-2)

LUCAS, MARCIA C. (Q-2)

PRATT, JOHN C. (Q-2)

53-6

ASSAY OF PLUTONIUM METAL BY GAMMA

SPECTROMETRY AND CALORIMETRY.

LOS ALAMOS,

1984. 20P. (LA-09879-MS)

BOWERSOX, DAVIDF. (MST-11)

WAGNER, RAYMOND P. (MST-12)
53-7

ASSESSMENT OF COMPUTER SECURITY

EFFECTIVENESS FOR SAFE PLANT OPERATION.

TRANS. AM. NUCL. SOC.

V.46, P.625-6. 1984.

SMITH, SUZANNE T. (Q-8)

LM, J. J.

53-8

AUTOMATIC MATERIAL IDENTIFIER.

INSTITUTE OF NUCLEAR MATERIALS

MANAGEMENT. ANNUAL MEETING, 25TH,

1984, COLUMBUS. PROC.

IN - NUCLEAR MATERIALS MANAGEMENT,

V.13, P.154-7. 1984

ERKKILA, BRUCE H. (TT-1)

53-9

CERENKOV METHODOLOGY FOR MONITORING IRRADIATED REACTOR FUEL.

TIC.

1984. 6P. MN (LA-UR-84-1437)

NICHOLSON, NICHOLAS (Q-2)

DOWDY, EDWARD J. (Q-2)

53-10

COINCIDENCE MEASUREMENTS OF FFTF

BREEDER FUEL SUBASSEMBLIES.

LOS ALAMOS.

1984. 22P. (LA-09902-MS)

ECCLESTON, GEORGE W. (Q-1)

FOLEY, JOHN E. (Q-1)

KRICK, MERLYN S. (Q-1)

MENLOVE, HOWARD O. (Q-1)

GORIS, P.

RAMALHO, A.

53-11

COMPARISON OF PREDICTED AND MEASURED

PULSED - COLUMN PROFILES AND

INVENTORIES.

TRANS. AM. NUCL PHYS.

V.45, P.28-9. 1983

ALSO PUBLISHED IN:

TIC,

1983. 13P. MN (LA-UR-83-3299)

OSTENAK, CARL ANDREW (Q-4)

CERMAK, A.F.

53-12

CUMULATIVE ERROR MODELS FOR THE TANK CALIBRATION PROBLEM

TIC.

1983. 9P. MN (LA-UR-63-2035)

GOLDMAN, MARON S. (Q-4)

ANDERSON, G. L

WEBER, JOE
53-19

DEADTIME REDUCTION IN THERMAL NEUTRON COINCIOENCE COUNTER.

LOS ALAMOS,

1984. 20P. (LA-09936-MS)

SWANSEN, JAMES E. (Q-1)

53-14

DEMONSTRATION OF NEAR - REAL - TIME

ACCOUNTING - THE AGNS 1980-81

MINIRUNS.

LOS ALAMOS,

1984. 248P. (LA-09942)

DAYEM, HASSAN ALY (Q-4)

BAKER, ALICE LELIA (Q-4)

COBB, DONALD D. (ESS-7)

HAKKILA, E. ARNOLD (Q-4)

OSTENAK, CARL ANDREW (Q-4)

63-15

DESIGN AND EVALUATION OF AN INTEGRATED

SAFEGUARDS SYSTEM - PRINCIPLES.

INSTITUTE OF NUCLEAR MATERIALS

MANAGEMENT. ANNUAL MEETING, 25TH,

1984, COLUMBUS. PROC.

IN - NUCLEAR MATERIALS MANAGEMENT,

V.13, P.348-55. 1984.

MARIIN, JACK T. (Q-4)

COULTER, C. ALTON (Q-4)

GUTMACHER, R. G. (Q-4)

WHITTY, WILLAM J. (Q-4)

53-16

DESIGN AND EVALUATION OF AN INTEGRATED

SAFECUARDS SYSTEM YOLUME 1. PRINCIPLES.

LOS ALAMOS,

1884. 121P. (LA-09964, VOL 1)

MARKIN, JACKT. (Q-4)

COULTER, C. ALTON (Q-4)

GUTMACHER, R. G. (0-4)

THOMAS, CHARLES C., JR. (Q-4)

WHITTY, WLLIAM J. (Q-4)

63-17

DESIGN AND EVALUATION OF AN INTEGRATED

SAFEGUARDS SYSTEM. YOLUME 2 - EXAMPLE APPLICATION OF THE PRINCIPLES

LOS ALAMOS

1984. 66P. (LA-09964, VOL 2)

COULTER, C. ALTON (O-4)

MARKIN, JACKT. (Q-4)

$53-18$

DETECTABILTY LIMITS AND PRECISION

FOR BHUFFLERS.

LOS ALAMOS.

1984. 10P. (LA-10158-MS)

CRANE, T.W. (Q-1) 
53-19

DETERMINATION OF PLUTONIUM ISOTOPIC RATIOS BY USING LOW - ENERGY GAMMA - RAY SPECTROSCOPY.

TIC,

1983. 8P. MN (LA-UR-83-3386)

LI, T.K. (Q-1)

$53-20$

DEVELOPMENT OF AN ENRICHMENT MONITOR FOR THE PORTSMOUTH GCEP.

TIC,

1983. 6P. MN (LA-UR-63-3298)

STRITTMATTER, RICHARD B. (Q-1)

STOVALL, LEONARD A. (Q-1)

SPRINKLE, JAMES K., JR. (Q-1)

53-21

DIRECT FISSILE ASSAY OF HIGHLY ENAICHED

URANIUM HEXAFLUORIDE USING RANDOM

SELF-INTERROGATION AND NEUTRON

COINCIDENCE RESPONSE.

TIC,

1983. 20P. MN (LA-UR-83-3266)

STEWART, JAMES E. (Q-1)

MENLOVE, HOWARD O. (Q-1)

$53-22$

EFFICIENCY OF 7.62 - CM BISMUTH

GERMANATE SCINTILLATORS.

NUCL. INSTRUM. METHODS,

V.221.P.378-83. 1984:

MOSS, CALVN E. (Q-2)

TISINGER, E. W. (Q-2)

HAMM, MARIANNE E. (Q-2)

$53-23$

ELECTRON ACCELERATOR BASED SYSTEM FOR ASSAY OF TRANSURANIC WASTE BARRELS.

LNEAR ACCELERATOR CONFERENCE

1984, SEEHEIM. PROC., P.427-9.

1984. (GSI-84-11)

ALSO PUBLISHED IN:

TIC,

1984. 31P. MN (LA-UR-84-0200)

KOCIMSKI, S. M

FRANKS, L. A.

CALDWELL, JOHN T. (Q-2)

CLOSE, DONALD A. (Q-2)

KUNZ, WALTER E. (Q-2)

MORGADO, RICHARD E. (Q-2)

BIERI, JOHN M. (Q-2)

53-24

ELECTRONICS MANUAL FOR THE FAST FACILITY

DELAYED NEUTRON INTERROGATOR ASSAY

SYSTEM.

LOS ALAMOS,

1984. 77P. (LA-09850-M)

VAN LYSSEL, THOMAS R. (Q-1)

ECCLESTON, GEORGE W. (Q-1)

53-25

ESTIMATION METHODS FOR PROCESS HOLDUP

OF SPECIAL NUCLEAR MATERIALS.

LOS ALAMOS

1984. 118P. (LA-10038)

PILLAY, K. K. S. (Q-4)

PICARD, RICHARD R. (S-1)

MARSHALL, ROBERT S. (Q-4)
53-26

ESTIMATION METHODS FOR SPECIAL NUCLEAR

MATERIALS HOLDUP

INSTITUTE OF NUCLEAR MATERIALS

MANAGEMENT. ANNUAL MEETING, 25TH,

1984, COLUMBUS. PROC.,

IN - NUCLEAR MATERIALS MANAGEMENT

V.13, P.60-5. 1984.

PILLAY, K. K. S. (Q-4)

PICARD, RICHARD R. (S-1)

53-27

EXPERIENCE USING A SPENT - FUEL

MEASUREMENT SYSTEM.

TIC,

1983. 8P. MN (LA-UR-83-1934)

PHILLIPS, JOHN R. (Q-1)

BOSLER, GERALD E. $(Q-1)$

HALBIG, JAMES K. (Q-1)

KLOSTERBUER, SHIRLEY F. (Q-1)

MENLOVE, HOWARD O. (Q-1)

RINARD, PHILLIP M. (Q-1)

53-28

FAST FACILITY SPENT - FUEL AND WASTE

ASSAY INSTRUMENT.

TIC,

1983. 16P. MN (LA-UR-83-3382)

ECCLESTON, GEORGE W. (Q-1)

JOHNSON, SUSAN S. (Q-1)

MENLOVE, HOWARD O. $(Q-1)$

VAN LYSSEL, THOMAS R. (Q-1)

BLACK, D.

CARLSON, B.

DECKER, L

ECHO, MAURICE W.

$53-29$

FINE TUNING OF A MEASUREMENT CONTROL PROGRAM AT THE LOS ALAMOS NATIONAL LABORATORY.

TRANS. AM. NUCL. SOC.,

V.45, P.56-8. 1983.

ALSO PUBLISHED IN:

TiC,

1983. 8P. MN (LA-UR-83-3392) GOLDMAN, AARON S. (Q-4) MARSHALL, ROBERT S. (Q-4)

53-30

GAMMA - RAY DETECTORS FOR INTELLIGENT,

HAND - HELD RADIATION MONITORS.

IEEE TRANS. NUCL. SCI.,

V.NS-31, P.664-7. 1984.

FEHLAU, PAUL E. (Q-2)

53-31

GAMMA - RAY DETECTORS FOR INTELLIGENT, HAND - HELD RADIATION MONITORS

TIC.

1983. 5P. MN (LA-UR-83-2909)

FEHLAU, PAULE. (Q-2)

53-32

GEOCHEMICAL ATLAS OF ALASKA.

LOS ALAMOS

1983. 57P. (LA-09897-MS)

WEAVER, THOMAS A. (ESS-3)
53-33

HEALTH PHYSICS APPLICATIONS OF NUCLEAR

SAFEGUARDS RADIATION MONITORS.

TIC.

1984. 9P. MN (LA-UR-83-3384)

FEHLAU, PAUL E. (Q-2)

DVORAK, ROBERT F. (HSE-1)

53-34

HIGH SENSITIVITY BULK TRU WASTE ASSAY SYSTEMS.

TRANS. AM. NUCL. SOC.,

V.46, P.311-2. 1984.

CALDWELL, JOHN T. (Q-2)

BERNARD, WILLIAM (Q-2)

BIERI, JOHN M. (O-2)

CLOSE, DONALD A. (Q-2)

FRANCE, STEPHEN W. (Q-2)

HASTINGS, RAY D. (Q-2)

HERRERA, GABAIEL CLARENCE (Q-2)

KUCKERTZ, THOMAS H. (Q-2)

KUNZ, WALTER E. (Q-2)

PRATT, JOHN C. (Q-2)

SHUNK, EDWARD R. (Q-2)

$53-35$

INTERNATIONAL TRAINING COURSE ON IMPLEMENTATION OF STATE SYSTEMS OF ACCOUNTING FOR AND CONTROL OF NUCLEAR MATERIALS. OCTOBER 17 - NOVEMBER 4, 1983 SANTA FE, NEW MEXICO AND RICHLAND, WASHINGTON

LOS ALAMOS,

1984. PAGED IN SECTIONS. (LA-10135-C)

HATCHER, CHARLES R. (Q-1)

SMITH, HASTINGS A., JR. (Q-1)

53-36

LITTLE BOY NEUTRON SPECTRUM BELOW

1 MEV.

TIC.

1984. 9P MN (LA-UR-83-3329)

EVANS, ALBERT E., JR. (Q-2)

53-37

MAINTAINING A HIGH 4.4 - MEV GAMMA - RAY OUTPUT RELATIVE TO TOTAL NEUTRON OUTPUT FROM AN AMERICIUM 241/BERYLLIUM SOURCE.

HEALTH PHYS.

V.46, P.474-6. 1984

ATWATER, HENAY F. (Q-2)

53-38

MATERIALS CONTROL AND ACCOUNTING

(MC AND A) - THE EVOLUTIONARY PRESSURES.

TIC,

1983. 5P. MN (LA-UR-83-3403)

ABSTRACT PUBLISHED IN:

TRANS. AM. NUCL. SOC.

V.45, P.52. 1983

SHIPLEY, JAMES P., JR. (Q-DO/SG) 
50-39

MATERIALS LOSS-DETECTION SENSITIVITIES USING PROCESS-GRADE MEASUREMENTS AT AGNS BNFP.

TIC,

1983. 7P. MN (LA-UR-83-3383)

DAYEM, HASSAN ALY (Q-4)

HAKKILA, E. ARNOLD (Q-4)

BAKER, ALICE LELIA (Q-4)

COBB, DONALD D. (ESS-7)

53-40

MATRIX EFFECTS FOR DIFFERENTIAL

DIE-AWAY WASTE ASSAYS.

TRANS. AM. NUCL. SOC.,

V.46, P.175-6. 1984.

KUNZ, WALTER E. (Q-2)

CALDWELL, JOHN T. (Q-2)

HASTINGS, RAY D. (Q-2)

HERRERA, GABRIEL CLARENCE (Q-2)

SHUNK, EDWARD R. (Q-2)

53-41

MEASUREMENT CONTROL PROGRAM FOR NDA INSTRUMENTS.

TIC,

1983. 5P. MN (LA-UR-83-3252)

HSUE, SIN-TAO (Q-5)

MARKS, THOMAS (MP-1)

53-42

MEASUREMENTS OF URANIUM HOLDUP IN AN OPERATING GASEOUS DIFFUSION ENRICHMENT PLANT.

TIC.

1983. 14P. MN (LA-UR-83-3381)

AUGUSTSON, RONALD H. (Q-3)

WALTON, RODDY B. (P-15)

HARRIS, $R$.

HARBARGER, W.

HICKS, J.

TIMMONS, G.

SHISSLER, D

TAYLOE, $R$

JONES, $S$.

FIELDS, L.

53-43

MODELING AND SIMULATION FOR PROCESS

AND SAFEGUARDS SYSTEM DESIGN.

TIC

1983. 8P. MN (LA-UR-83-3344)

ABSTRACT PUBLISHED IN

TRANS. AM. NUCL. SOC.

V.45, P.35. 1983.

GUTMACHER, R. G. (Q-4)

KERN, EDWARD A. (Q-4)

DUNCAN, D. R.

BENECKE, M.W.
53-44

MULTI - ISOTOPIC GAMMA - RAY ASSAY

SYSTEM FOR ALPHA - CONTAMINATED WASTE

TIC.

1983. 6P. MN (LA-UR-83-1931)

CLOSE, DONALD A. (H-4)

PRATT, JOHN C. (Q-2)

CALDWELL, JOHN T. (Q-2)

KUNZ, WALTER E. (Q-2)

SCHULTZ, F.J.

HAFF, K. W.

53-45

NEW GENERATION LOW POWER RADIATION

SURVEY INSTRUMENTS.

TIC,

1983. 5P. MN (LA-UR-83-2942)

WAECHTER, DAVID A. (E-8)

BJARKE, GEORGE O. (IT-1)

WOLF, MICHAEL ALLEN (IT-1)

TRUJILLO, FAUSTIN (IT-1)

UMBARGER, C. JOHN (IT-1)

53-46

OPERATION OF AUTOMATED NDA INSTRUMENTS

FOR IN - LINE HEU ACCOUNTING AT Y - 12

TIC.

1983. 30P. MN (LA-UR-83-3320)

RUSSO,P. A. (Q-1)

STRITTMATTER, RICHARD B. (Q-1)

SANDFORD, ELSIE L. (Q-1)

JETER, I. W.

MC CULLOUGH, E.

BOWERS, G. L.

53-47

PAJARITO MONITOR - A HIGH-SENSITIVITY

MONITORING SYSTEM FOR HIGHLY ENRICHED

URANIUM.

INSTITUTE OF NUCLEAR MATERIALS

MANAGEMENT. ANNUAL MEETING, 25TH,

1984, COLUMBUS. PROC.

IN - NUCLEAR MATERIALS MANAGEMENT,

V.13, P 66-72. 1984.

FEHLAU, PAUL E. (Q-2)

COOP, K. L. (Q-2)

GARCIA, CLEMENTE (Q-2)

MARTINEZ, JAKE (Q-2)

53-48

PLUTONIUM NITRATE BOTTLE COUNTER MANUAL.

LOS ALAMOS

1984. 22P. (LA-10009-MS)

MENLOVE, HOWARD O. (Q-1)

ADAMS, EDWIN L. (Q-1)

HOLBROOKS, ORVILLE R. (Q-1)

$53-49$

PROCESS - (INTERNATIONAL) SAFEGUARDS

INTERFACE AT THE PORTMOUTH GCEP.

TRANS. AM. NUCL. SOC.,

V.45, P.8. 1983

ALSO PUBLISHED IN:

TIC,

1983. 6P. MN (LA-UR-83-3325)

TAPE, JAMES W. (Q-1)

STRITTMATTER, RICHARD B. (Q-1)

BAKER, ALICE LELIA (Q-4)
53-50

SAFEGUARDS AND SECURITY PROGRESS

REPORT, JANUARY - DECEMBER 1983.

LOS ALAMOS,

1984. 84P. (LA-10170-PR)

SMITH, DARRYL B. (Q-DO/SG)

53-51

SEQUENTIAL PROBABILITY RATIO

CONTROLLERS FOR SAFEGUARDS

RADIATION MONITORS.

TIC.

1984. 4P. MN (LA-UR-84-1436)

FEHLAU, PAUL E. (Q-2)

COOP, K. L. (Q-2)

NIXON, KERMIT V. (Q-2)

53-52

SMARTER RADIATION MONITORS FOR

SAFEGUARDS AND SECURITY.

TIC,

1983. 8P. MN (LA-UR-83-2036)

FEHLAU, PAUL E. (Q-2)

PRATT, JOHN C. (Q-2)

MARKIN, JACK T. (Q-4)

SCURRY, T. J.

53-53

SYSTEM FOR IMAGING PLUTONIUM THROUGH HEAWY SHIELDING.

TIC.

1984. 6P. MN (LA-UR-94-1390)

KUCKERTZ, THOMAS H. (E-8)

CANNON, THOMAS M. (IT-3)

FENIMORE, EDWARD E. (ESS-9)

MOSS, CALVIN E. (Q-2)

NIXON, KERMIT V. (Q-2)

53-54

SYSTEM FOR MEASURING TRU MATERIALS

IN A THREE MILE ISLAND DEMINERALIZER

TRANS. AM. NUCL. SOC.

V.46, P.314. 1984

HALBIG, JAMES K. (Q-1)

BOSLER, GERALD E. (Q-1)

PHILLIPS, JOHN R. (Q-1)

RINARD, PHILLIP M. (Q-1)

53-55

THREAT OF THE NEO-LUDDITES.

APMS CONTROL AND INTERNATIONAL

SECURITY, R. KOLKOWICZ, N. JOECK,

EDS., P.68-76. WESTVIEW, 1984.

(327.174/A734COC)

KERR, DONALD M., JR. (DIR-O)

53-56

UNFOLDING BISMUTH - GERMANATE PULSE HEIGHT DISTRIBUTIONS TO DETERMINE

GAMMA - RAY FLUX SPECTRA AND DOSE RATES.

NUCL. INSTRUM. METHODS,

V.219, P.558-64. 1984.

MOSS, CALVIN E. (Q-2)

DOWDY, EDWARD J. (Q-2)

EVANS, ALBERT E., JR. (Q-2)

HAMM, MARIANNE E. (Q-2)

LUCAS, MARCIA C. (Q-2)

SHUNK, EDWARD R. (Q-2) 
53-57

UNIVERSAL FAST BREEDER REACTOR

SUBASSEMBLY COUNTER MANUAL.

LOS ALAMOS

1984. 48P. (LA-10226-M)

MENLOVE, HOWARD O. (Q-1)

ECCLESTON, GEORGE W. (Q-1)

SWANSEN, JAMES E. (Q-1)

GORIS, $P$.

ABEDIN-ZADEH, $R$.

RAMALHO, A.
53-58

USE OF CALIBRATION STANDARDS AND THE CORRECTION FOR SAMPLE SELF - ATTENUATION. IN GAMMA - RAY NONDESTRUCTIVE ASSAY. LOS ALAMOS,

1984. 33P. (LA-10045)

PARKER, JACK L. (Q-1)
53-59

VIOLINIST - SOPHISTICATED PLUTONIUM

FIELD MONITOR.

TIC,

1983. 3P. MN (LA-UR-83-2941)

UMBARGER, C. JOHN (TT-1)

WOLF, MICHAEL ALLEN (IT-1)

TRUJILLO, FAUSTIN (IT-1) 
55. SOLAR THERMAL

$55-1$

RESONANT PHOTOEMISSION STUDIES OF

NARROW-BAND MATERIALS.

STANFORD SYNCHROTRON RADIATION LAB.

ACTIVITY REPORT FOR APRIL 1, 1983-

DECEMBER 31, 1983. K. CANTWELL, ED.,

P.IX-44-6. SSRL, 1983 (SSRL-84/01)

ALLEN, JAMES $W$.

OH, SEJUNG

ELLIS, WALTON P. (CHM-2)

COX, LAWRENCE E. (MST-13)

COWAN, ROBERT D. (T-4)

LINDAU, I. 


\section{THEORETICAL PLASMA PHYSICS}

56-1

ACOUSTICALLY COMPENSATED TWO WAVELENGTH INTERFEROMETRY FOR PLASMA DENSITY MEASUREMENTS - ONE-DIMENSIONAL THEORY AND APPLICATIONS.

LOS ALAMOS,

1984. 17P. (LA-09880-MS)

BUCHENAUER, C. JERALD (CTR-7)

56-2

ANALYTICAL SOLUTIONS OF CURL B EQUALS LAMBDA B HAVING SEPARATRICES FOR GEOMETRIES WITH ONE IGNORABLE COORDINATE.

PHYS. FLUIDS,

V.27, P.1677-85. 1984.

TURNER, LEAF (CTR-6)

$56-3$

ENHANCED PENETRATION OF FIELD - ALIGNED CURRENTS INTO A LORENTZ PLASMA IN A STOCHASTIC MAGNETIC FIELD.

J. APPL. PHYS.,

V.56, P.2023-5. 1984

JACOBSON, ABRAM R. (CTR-2)

MOSES, RONALD W., JR. (CTR-6)

$56-4$

EXACT INVARIANTS QUADRATIC IN THE

MOMENTUM FOR A PARTICLE IN A THREE -

DIMENSIONAL ELECTROMAGNETIC FIELD.

J. MATH. PHYS.

V.25, P.1139-42. 1984.

LEWIS, H. RALPH, JR. (CTR-6)

56-5

EXACT TIME - DEPENDENT SOLUTIONS OF

THE VLASOV - POISSON EQUATIONS.

PHYS. FLUIDS,

V.27, P.192-6. 1984.

LEWIS, H. RALPH, JR. (CTR-6)

SYMON, KEITH R. (CTR-6)
$56-6$

FIELD - ERROR INDUCED TAANSPORT IN A

PURE ELECTRON PLASMA COLUMN.

PHYS. FLUIDS,

V.27, P.1427-33. 1984

KEINIGS, RHONALD K. (CTR-6)

56-7

HAMILTONIAN STRUCTURE OF FIELD LINE EQUATIONS.

LOS ALAMOS,

1984. 6P. (LA-10048-MS)

CHANNELL, PAUL J. (AT-6)

56-8

INADEQUACIES OF FINITE LARMOR RADIUS

TREATMENTS OF THE INTERNAL TILTING

INSTABILTTY IN FIELD-REVERSED

CONFIGURATIONS.

PHYS. FLUIDS,

V.27, P.2151-5. 1984.

SCHWARZMEIER, JAMES L. (CTR-6)

SEYLER, CHARLES E., JR. (CTR-6)

56-9

INAPPROPRIATENESS OF THE FINITE LARMOR RADIUS MODEL FOR THE TILTING MODE IN

FIELD REVERSED CONFIGURATIONS.

TIC.

1984. 5P. MN (LA-UR-84-0632)

SCHWARZMEIER, JAMES L (CTR-6)

SEYLER, CHARLES E., JR. (CTR-6)

56-10

MAGNETIC FIELD PERTURBATIONS DUE TO A HOLE IN A CONDUCTING WALL ON THE ZT4OM REVERSED-FIELD PINCH.

J. APPL. PHYS.,

V.56, P.2017-21. 1984. HOWELL, ROBERT B. (CTR-2) VOGEL, HERBERT F. (CTR-4)
56-11

MAGNETIC FIELD TOPOLOGIES OF THE RELAXED STATES OF TURBULENT DISCHARGES.

TIC,

1983. 19P. MN (LA-UR-83-2691)

TURNER, LEAF (CTR-6)

56-12

MHD EQUILIBAIUM AND STABILTY OF

ROTATING FRCS WITH EXCLUDED MULTIPOLE

FIELDS.

TIC.

1984. 5P. MN (LA-UR-84-0633)

SPENCER, ROSS L (CTR-6)

56-13

ONE - DIMENSIONAL TRANSPORT CODE FOR

FIELD - REVERSED CONFIGURATIONS.

TIC.

1984. 5P. MN (LA-UR-84-0631)

CARAMANA, EDWARD J. (CTR-6)

HSIAO, MING Y. (CTR-6)

SCHWARZMEIER, JAMES L. (CTR-6)

56-14

THERMAL INSTABILITIES IN THE EDGE

REGION OF REVERSED - FIELD PINCHES.

LOS ALAMOS,

1984. 30P. (LA-10029-MS)

GOEDERT, JOAO

MONDT, JOHANNES P. (CTR-6)

56-15

TRANSPORT, STABILITY AND RELAXATION OF

A SPHEROMAK.

TIC,

1984. 3P. MN (LA-UR-84-0630)

SGRO, ANTHONY G. (CTR-6)

MARKLIN, GEORGE J. (CTR-6)

MIRIN, ART A. 


\section{TRANSPORTATION OF PROPERTY AND NUCLEAR MATERIALS}

$57-1$

CARGO RESPONSE TO RAILCAR IMPACT AND TIEDOWN LOAD ANALYSIS.

TIC,

1978. 10P. MN (LA-UR-78-1210)

ALSO PUBLISHED IN:

INTL. SYMPOSIUM ON PACKAGING AND

TRANSPORTATION OF RADIOACTIVE

MATERIALS, 5TH, 1978, LAS VEGAS.

PROC., P.391-9. THE CONF. 1978.

(363.179/161PA/5TH/1978/NOL.1)

BARTHOLOMEW, ROBERT J. $(W X-8)$
57-2

REVIEW OF CRITERIA FOR NUCLEAR

CRITICALITY SAFETY CONTROL IN

TRANSPORTATION.

INTL. SYMPOSIUM ON PACKAGING AND

TRANSPORTATION OF RADIOACTIVE

MATERIALS, 5TH, 1978, LAS VEGAS.

PROC., P.561-70. THE CONF. 1978.

(363.179/161PA/5TH/1978/NOL.2)

THOMAS, JOET.

SMITH, DAVID R. (HSE-6) 


\section{AUTHOR INDEX}

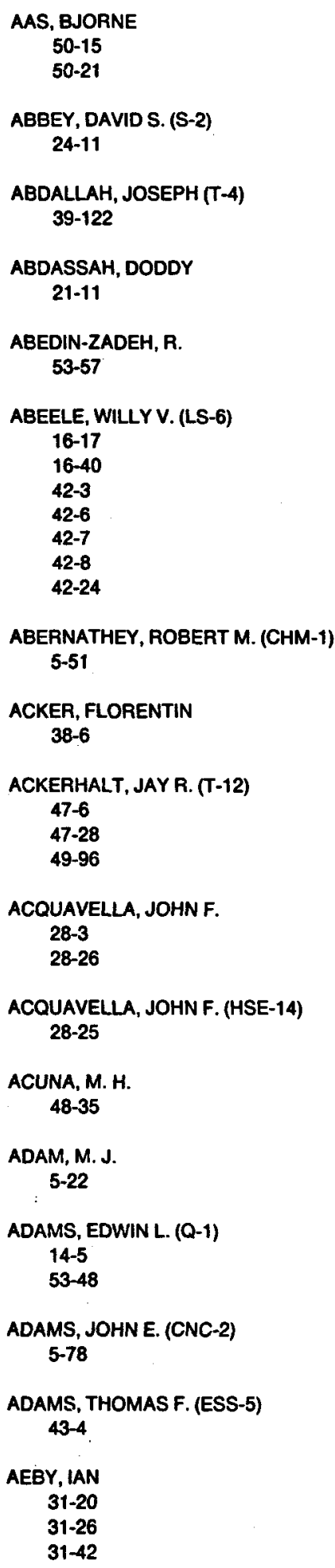

AERTS, ADRIAN T. (T-5)

$$
\text { 47-24 }
$$

AGNEW, LEWIS E., JR. (MP-DO) 44-7

AHARONOV, Y. 50-44

AHRENS, THOMAS J.

16-16

AIKEN, R. L.

5-60

AIKIN, IRENE C. (HSE-1) 28-19

AKPORIAYE, EMMANUEL T. $2-16$

ALBRIGHT, JAMES N. (ESS-4) 21-2

22-5

ALCORN, J. S. $37-4$

ALCOUFFE, RAYMONDE. (X-6) 39-118

$39-119$

ALDE, D. M. 50-92

ALDISSI, MAHMOUD (MST-6) 38-40

ALDRICH, CHARLES H. $(X-1)$ 48-56

ALDRICH, MERRITT J. (ESS-1) 16-24

ALDRIDGE, JACK P. (NSP/AWT) 39-5

ALEXANDER, $C$. 50-130

ALKIRE, RANDALL W. (P-8) 5-82

ALLEN, JAMES $W$. 55-1

ALLEN, JAMES W. (P-APO) 47-12

ALLER, H.D. 48-71
ALLER, M. F.

48-71

ALLISON, PAUL W. (AT-2)

44-36

44-58

ALLISON, S.W.

26-8

49-157

ALLRED, JOHN C. (MP-DO) 44-46

ALRICK, KEITH R. (P-15) 14-62

ALSTER, JONAS

50-4

50-24

50-107

ALT, WOLFGANG 2-6

ALTSEIMER, JOHN H. (S-4) 22-2

AMANN, JAMES F. (MP-10) 39-65

$50-10$

ANDER, MARK E. (ESS-3)

16-5

22-3

ANDERSON, ALLAN B. (M-1) 3-2

ANDERSON, CHARLES A. (Q-13) 38-7

ANDERSON, DAVID F. (H-1) 14-29

14-66

28-2

ANDERSON, G. L. 53-12

ANDERSON, GRAYDON K. (CHM-3) 47-56

ANDERSON, JAMES L. (MST-3) 18-16

ANDERSON, JAMES WAYNE (C-8) 39-85

ANDERSON, RICHARD C. (ESS-8) 48-16

48-32 
ANDRADE, ANTHONY (X-2) 50-87

ANDRAE, RICHARD W. (Q-6) 39-116

ANDREJKO, MICHAEL J. 5-6

ANNESTRAND, S. A. 37-15

ANSALDO, E.J. 49-87

APEL, CHARLES T. (CHM-1) 5-11

APEL, CHARLES T. (CMB-1) 5-10

APPERT, QUENTIN D. (P-5) 30-16

ARAKI, CLIFFORD T. (LS-2) 14-10

ARCHULETA, JACOBO R. (G-4) 31-1

ARDEN, BRUCE 39-46

ARENDT, PAUL N. (MST-6) 38-1

ARGO, HAROLD V. (ESS-9) 16-22

ARGO, PAUL E. (ESS-7) 48-23

ARMIJO, VONCILLE M. (INC-11) 5-28

ARMSTRONG, DALE D. (AT-4) 44-55

44-64

ARMSTRONG, WILLIAM T. (CTR-3)

17-7

17-14

$17-36$

ARNEY, BARBARA H. (ESS-1)

22-6

22-7

ARNOLD, JAMES R. 48-54

ARTHUR, EDWARD D. (T-2)

18-7

$50-7$

50-35
ARVIND,

$39-46$

ASBRIDGE, JOHN R. (ESS-8)

48-31

48-88

ASHERY, D.

50-93

ASPREY, LARNED B. (CNC-3) 5-40

ASPREY, MARGARET $W .(x-7)$ 36-2

ATENCIO, J. D. (Q-2)

14-3

ATENCIO, L. G. (MP-10) 28-17

ATWATER, HENRY F. (Q-2) 53-37

ATZMON, MICHAEL 50-64

AUBLE, $R$.

50-94

AUCHAMPAUGH, GEORGE F. (P-3) 50-55

$50-80$

AUER, I. P. 49-81

AUERBACH, NAFTALI
$50-38$
$50-39$
$50-89$
$50-115$
$50-125$

AUGUSTSON, RONALD H. (Q-3) 53-42

AWES, $T$.

50-45

AZIZI, A.

47-13

50-15

BAARS, RALPH E. (Q-7)

24-2

BABCOCK, C. D., JR. (Q-13) 24-1

BABCOCK, LUCIA M. (CHM-2)

5-26

5-27

5-79

BACHER, ANDY D. (P-7) 50-62
BAER, H. W. (MP-4)

50-4

50-9

50-24

50-126

BAER, HELMUT (MP-4)

50-67

50-97

BAILEY, DAVID J. (OS-4) 39-10

BAINES, D.W.T. 48-82

BAKER, ALICE LELIA (Q-4)

53-14

53-39

53-49

BAKER, DANIEL N. (ESS-8)

25-25

48-17

48-21

48-25

48-35

48-37

48-38

48-41

48-50

48-53

48-63

48-74

48-85

48-87

48-91

48-109

BAKER, DON A. (CTR-2)

$17-20$

17-29

BAKER, GEORGE A., JR. (T-11)

$39-39$

39-55

49-10

49-61

49-70

$51-18$

BAKER, WILFRED E. 24-1

BALCOMB, J. DOUGLAS (Q-DO)

29-2

29-3

29-5

29-10

29-12

29-13

BALCOMB, J. DOUGLAS (Q-13) 29-9

BALDWIN, GEORGE C. (P-3) 49-90 
BALDWIN, THOMAS S. (MP-7) 44-53

BALESTRINI, SILVIO J. (CNC-11) 49-16

BALL, GORDON C. 50-135

BALLARD, EVAN O. (P-12) 14-56

BAME, KAREN (LS-4) 2-60

BAME, SAMUEL J., JR. (ESS-8) 31-4

48-17

48-21

48-25

48-31

48-32

48-37

48-38

48-39

48-41

48-56

48-73

48-87

48-88

48-91

48-109

48-111

BANGERTER, ROGER O. (NSP/IF) 30-11

BARBEE, TROY $W$. 49-106

BARCLAY, JOHN A. (P-10)

14-20

14-39

14-40

49-4

49-46

49-73

BARDIN, S. W. (ESS-2)

5-6

BAREFIELD, J. E., II (CHM-4) 47-38

BARHAM, STEVEN S. 2-39

BARLETT, $M$.

50-59

BARLOW, D.

49-79

50-19
BARNES, CHRISTOPHER W. (CTR-5)

$$
\begin{aligned}
& 18-4 \\
& 18-8 \\
& 18-9 \\
& 18-10 \\
& 18-13
\end{aligned}
$$

BARNES, JAMES W. (Q-4) 43-3

BARNES, JAMES $W .(W X-4)$ 43-2

BARNES, JOHN W. (CNC-3) 5-33

BARNES, JOHN W. (INC-3) 5-32

$5-43$

BARNETT, CHARLES R. (IS-2) 25-22

BARR, DONALD W. (INC-11) 16-11

BARR, SUMNER (ESS-7) $16-41$

BARRACLOUGH, BRUCE L. (CMB-1) 31-2

BARRON, MICHAEL H. (CTR-9) $37-4$

BARTHELL, BARRY L. (MST-6) 14-11

BARTHELL, BARRY L. (MST-7) 14-46

BARTHOLDI, MARTY FRANK (LS-4) 2-12

2-15

2-26

BARTHOLOMEW, ROBERT J. (Q-12) 39-110

BARTHOLOMEW, ROBERT J. (WX-8) 57-1

BARTLETT, ROGER J. (P-14)

14-26

14-49

31-35

48-121

49-106

49-107

49-108

BARTLIT, JOHN R. (MST-3)

18-6

18-16

BARTMANN, RICHARD M. 16-12
BARTOE, J. D. F.

48-52

BARTS, EDWARD W. (Q-6) 33-18

BASKETT, FOREST (C-11) 39-46

BASSETT, BRITT (Q-7)

33-16

BATHKE, CHARLES G. (CTR-12)

18-2

18-11

BATLOGG, B

38-18

38-37

$38-49$

BATRA, RAVI (ESS-3) 21-2

BATTAT, MORRIS E. 18-2

18-18

BAUGHCUM, STEVEN L. (CHM-4) 5-41

BAUKE, WALTER (P-5)

30-4

30-17

44-38

BEATTIE, WILLARD H. (AP-4) 5-64

BEATTIE, WILLARD H. (CHM-4) 5-67

BEAUMONT, ARTHUR J. (MST-11) 25-23

BECKER, NAOMI M. (HSE-8)

16-29

16-44

BECKER, STEPHEN A. ( $\mathrm{X}-2)$ 48-6

48-100

BECKMAN, RICHARD J. (S-1)

5-51

28-5

$39-57$

50-52

BEGAY, FRED (P-4)

30-19

BEHRENS, ROBERT G. (CMB-3) 38-48 
BEHRENS, ROBERT G. (MST-3) 5-70
BELIAN, RICHARD D. (ESS-8)
48-17
48-50
48-51

\section{BELL, GEORGE I. (T-DO) 2-8 \\ $2-9$}

BELLUOMINI, STEPHEN G. 22-6

BELY-DUBAU, FRANCOISE 48-2

BENAGE, JOHN F., JR. (MP-11) 44-10

BENDER, CARL $M$.

$$
\text { 51-41 }
$$

BENDER, STEVEN C. (P-5) 30-16

BENDT, PHILIP J. (P-2) 50-83

BENECKE, M. W.

53-43
BENJAMIN, ROBERT F. (M-6) 14-6
$14-48$
14-50
49-11

BENJAMIN, TIMOTHY M. (INC-7) 16-14 48-69

BENNETT, BARD I., JR. (T-4) $49-40$

49-94

BENNETT, E. F. 9-12

BENNETT, JOEL G. (Q-13) 6-1

24-1

BENTLEY, GLENN E. (INC-3) 5-19 5-43

BENZIGER, THEODORE M. (WX-2) 5-42

BERARDINELLI, S.P. 28-4

BERG, DONNA (IS-4) 26-10

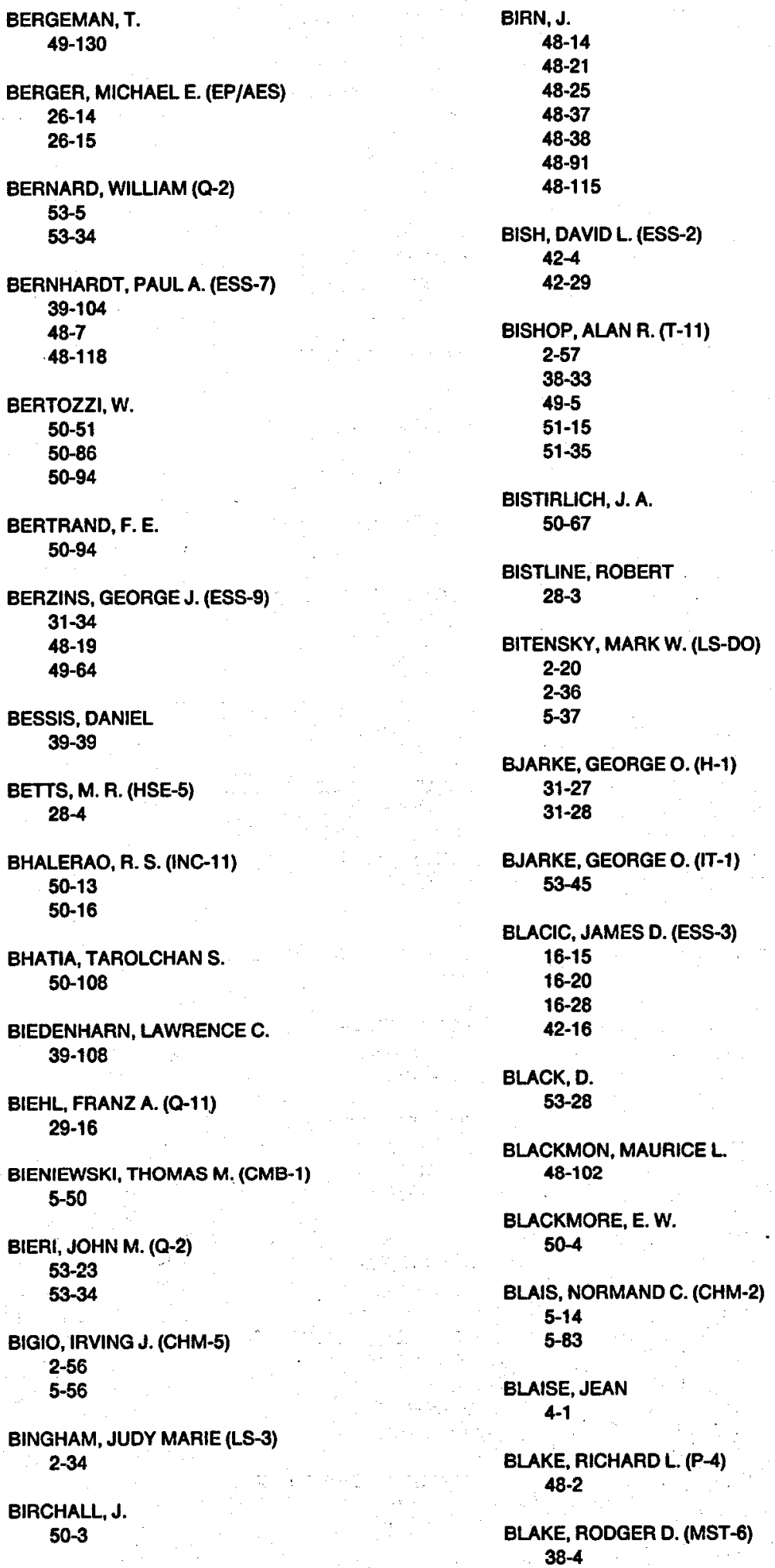




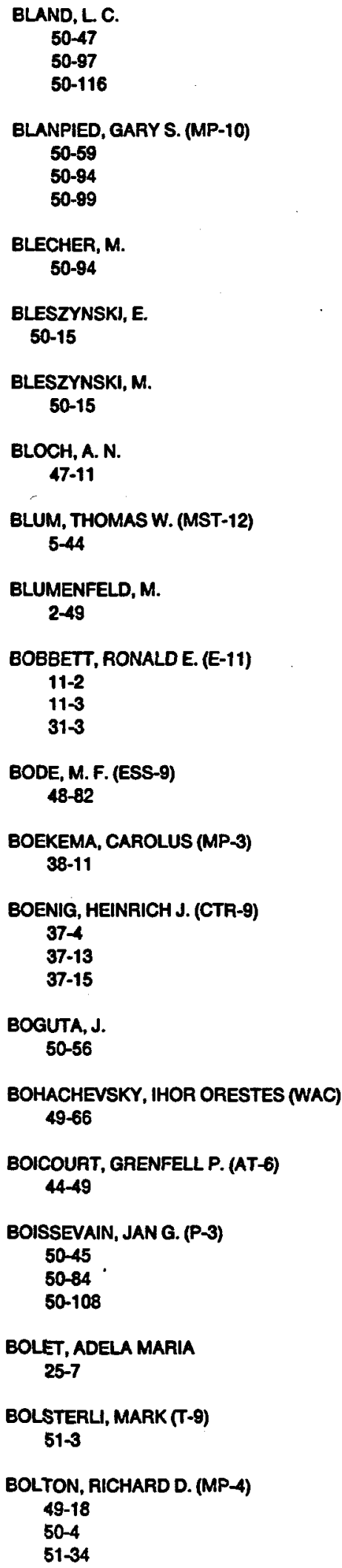

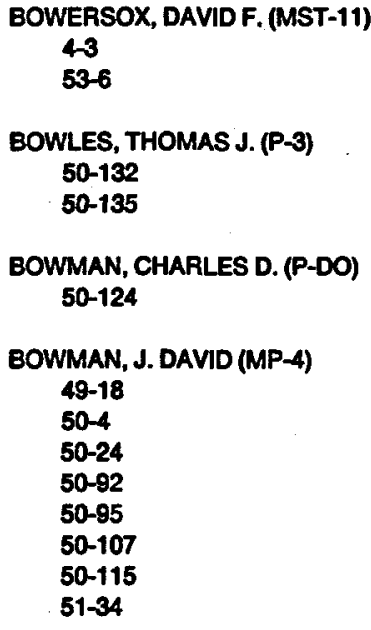

BRATTHWAITE, WILFED J. 50-99 


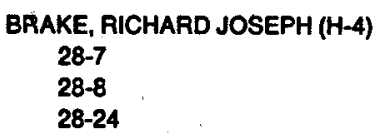

BRIXNER, BERLYN (M-5) 49-99

BROLLEY, JOHN E., JR. (P-14) 31-16

BRONISZ, STANLEY E. (MST-5) 40-11

40-12

40-13

$40-14$

40-15

40-16
BROWN, DAVID (E-5)

31-24

BROWN, DAVID R. (MST-6) 38-1

BROWN, J. MICHAEL

16-16

49-62

49-123

BROWN, LAURA B.

$$
\text { 5-15 }
$$

BROWN, LEE F. (ESS-5)

21-9

22-8

39-84

BROWN, MARK K. 50-97

BROWN, R. R. (E-5)

$37-4$

$39-33$

BROWN, R. R. (E-8)

14-10

$31-7$

31-19

BROWN, ROBERT D. (MP-7) 38-10

BROWN, RONALD E. (P-3)

50-3

50-32.

$50-48$

50-132

50-138

BROWN, WILBUR K. (P-15) " 48-46

BROWNE, JAMES C. 39-46

BROWNING, RICHARD V. (WX-11) 39-91

BROXTON, DAVID E. (ESS-2)

$$
\text { 42-29 }
$$

BRUECKNER, G.E. 48-52

BRUGGER, ROBERT M. (P-8) 31-38

BRUHWEILER, F.C. 48-71

BRUNDIGE, EOWARD L. (MST-11) 25-23

BRUNISH, WENDEE M. (T-6) 48-100
BRYANT, ERNEST A. (CNC-7) 42-20

BRYANT, HOWARD C. 49-130

BRYANT, LAWRENCE E., JR. (WX-3) 31-17

$31-18$

$31-39$

BRYSON, MAURICE C. (S-1)

24-13

25-5

39-87

BUBERNAK, JOSEPH (CHM-1) 5-57

BUCHENAUER, C. JERALD (CTR-7) 14-2

17-8

17-10

56-1

BUCHER, INGRID Y. (C-3) 39-107

BUCHHOLZ, JERRY R. (HSE-7) 42-13

BUCHSBAUM, STEVEN (P-10) 47-42

BUDEN, DAVID (DAD/NP) 40-10

BUHL, THOMAS E. (H-8) 26-10

BUMSTED, M. PAMELA (CHM-1) 2-27

BUNKER, MERLE E. (INC-5) 24-9

50-54

BUNKER, SUSAN S. (X-5) 48-3

48-5

BUONTEMPO. U. 47-57

BURKETT, MICHAEL W. (Q-6) 24-3 33-16

BURKHARDT, LOUIS C. (CTR-2) 17-23

BURKS, CHRISTIAN (T-10) 2-30

BURLESON, GEORGE R. 49-81 50-97 50-99. 


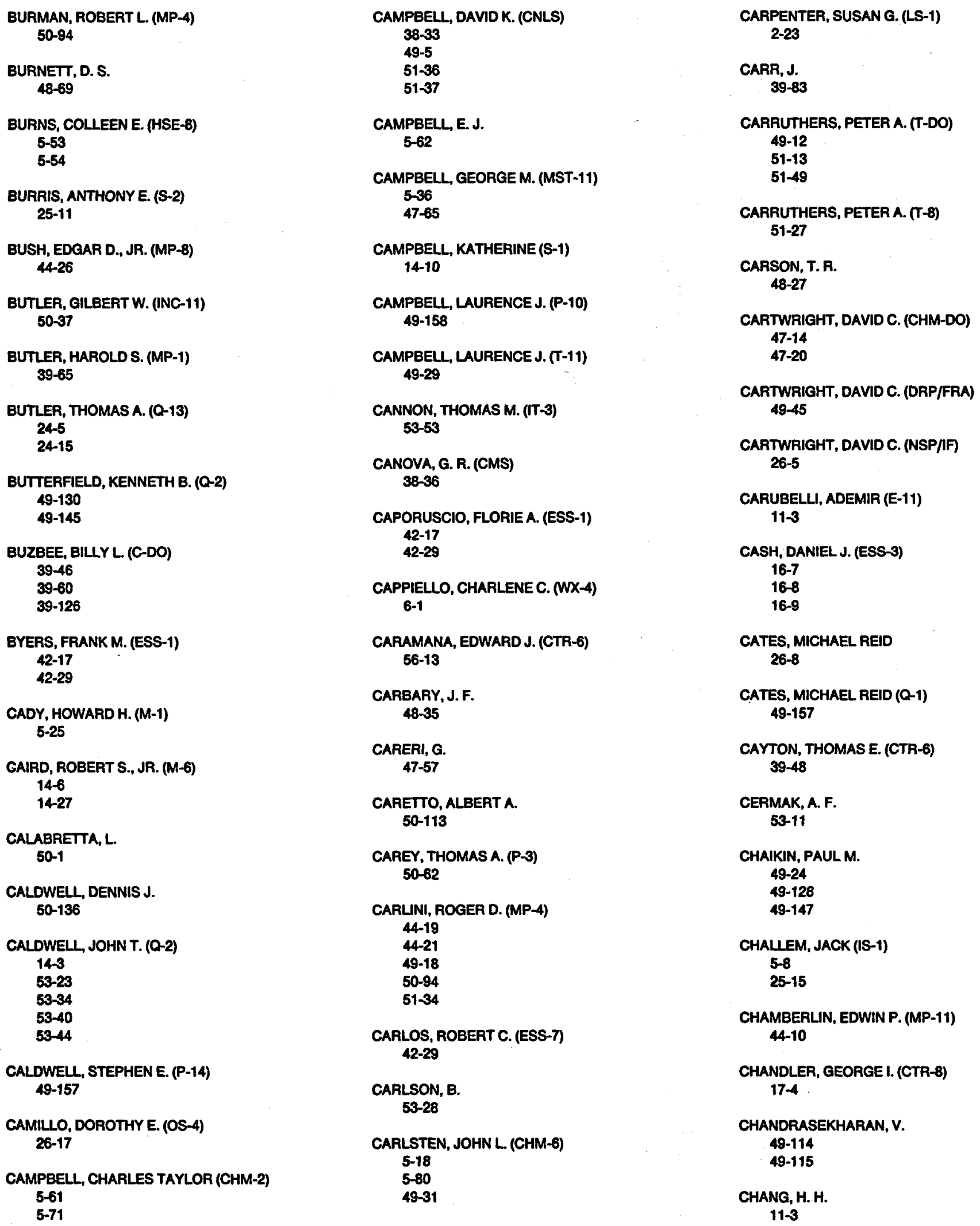

CAMPBELL, DAVIDK. (CNLS)

$38-33$

49-5

$51-36$

$51-37$

CAMPBELLL, E. J. 5-62

CAMPBELL, GEORGE M. (MST-11) 5-36 47-65

CAMPBELL, KATHERINE(S-1) 14-10

CAMPBELL, LAURENCE J. (P-10) 49-158

CAMPBELL, LAURENCE J. (T-11) 49-29

CANNON, THOMAS M. (TT-3) 53-53

CANOVA, G. R. (CMS) 38-36

CAPORUSCIO, FLORIE A. (ESS-1) 42-17 42-29

CAPPIELLO, CHARLENE C. $(W x-4)$ 6-1

CARAMANA, EDWARD J. (CTR-6) 56-13

CARBaRY, J. $F$.

48-35

CARERI, G. 47-57

CARETTO, ALBERT A. 50-113

CAREY, THOMAS A. (P-3) 50-62

CARLINI, ROGER D. (MP-4)

44-19

44-21

49-18

50-94

51-34

CARLOS, ROBERT C. (ESS-7) 42-29

CARLSON, B. 53-28

CARLSTEN, JOHN L (CHM-6)

5-18

$5-80$

49-31

CARPENTER, SUSAN G. (LS-1) 2-23

CARR, J. 39-83

CARRUTHERS, PETER A. (T-DO) 49-12

51-13

$51-49$

CARRUTHERS, PETER A. (T-8) 51-27

CARSON, T.R. 48-27

CARTWRIGHT, DAVID C. (CHM-DO) 47-14 47-20

CARTWRIGHT, DAVID C. (DRP/FRA) 49-45

CARTWRIGHT, DAVID C. (NSP/IF) 26-5

CARUBELLI, ADEMIR (E-11) $11-3$

CASH, DANIEL J. (ESS-3) 16-7

16-8 $16-9$

CATES, MICHAEL REID 26-8

CATES, MICHAEL REID (Q-1) 49-157

CAYTON, THOMAS E. (CTR-6) $39-48$

CERMAK, A. F. 53-11

CHAIKIN, PAUL M. 49-24 49-128 49-147

CHALLEM, JACK (IS-1) 5-8 25-15

CHAMBERLIN, EDWIN P. (MP-11) 44-10

CHANDLER, GEORGE I. (CTR-8) $17-4$

ChANDRASEKHARAN, $V$. 49-114 49-115

CHANG, H. H. $11-3$ 
CHANNELL, PAUL J. (AT-6)

44-13

44-30

56-7

CHAPLINE, GEORGE 51-23

CHAPLINE, GEORGE F. 49-159

ChASTAIN, R. E $37-4$

CHEIFETZ, ELI (P-9) $50-46$

CHEN, DAVID JEN-CHI (LS-3)

2-1

28-13

CHERNOFF, NAOMI 5-37

Cheseborough, ROBERT (P-10) 49-4

CHESTER, T. J. 48-81

CHEVALIER, ROgER A. 48-11

CHIANG, H. C. 50-96

CHIN, D. T. 5-17

$11-3$

CHOPPIN, GREGORY R. (INC-4) 5-1

CHRIEN, ROBERT E. (CTR-3) 17-14 $.17-36$

CHRISTENSEN, DANA CARL (MST-13) 5-52

CHRISTIE, J. M. 16-28

CLARK, DAVID A. (P-9) 44-38 49-130

CLARK, ROBERT EDWARD HOLMES (x-7) $47-43$

CLAWSON, CARL W. $49-87$

CLEMENTS, WILLIAM E. (ESS-7) 16-31 16-36
CLIFTON, DAVID G. (MST-12) 4-2

5-44

CLINARD, FRANK W., JR. (MST-5)

$37-2$

37-10

38-43

38-44

CLOSE, DONALD A. (H-4)

53-44

Close, DONALD A. (Q-2)

53-3

$53-23$

53-34

CLOUT, PETER N. (AT-3) 44-47

ClOVER, M. R. (P-2) 50-101

CLUFF, CORAN L. 32-3

COBB, DONALD D. (ESS-7) 53-14 53-39

CODDINGTON, PAUL 33-22 COFFELT, K. P. (CHM-1)
$40-1$

$40-8$

COHEN, JAMES S. (T-12) 47-29

49-59

COKAL, EDWARD J. (LS-6)

42-19

42-28

COLBURN, CHRISTOPHER W. 31-30

COLEMAN, J. G. 37-4

COLGATE, STIRLING A. (T-6)

48-48

$48-49$

48-103

48-110

COLLINS, LEE A. (T-4)

26-9

47-10

$47-33$

47-43

47-51

49-36
COLLINS, MAURICE W. (C-8)

39-75

$39-78$

COLOMBANT, $D$. 30-6

COLTON, EUGENE P. (MP-14) 44-61

44-62

52-1

COMLY, JACK C., JR. (X-1) 49-160

CONNELL, JOHN L. (ADP-2) 39-11

39-123

CONNELLEE, BARBARA G. (MST-11) 25-21

CONNER, JERRY P. (ESS-DO) 48-51

CONZETT, H. E. $50-3$

COOK, DENNIS R. 50-59

COOK, R. DENNIS (S-1) 39-25

COOK, THOMAS L. (ESS-5) 39-129

42-14

42-15

COOKE, D. W.

38-11

$47-37$

47-55

COOKE, D. W. (MP-3)

28-27

50-130

COOP, K. L. (Q-2)

28-19

$53-47$

53-51

COOPER, FREDERICK M. (T-8)

51-10

$51-29$

COOPER, MARTIN D. (MP-4)

49-18

$50-4$

$50-24$

$50-95$

50-107

$51-34$ 


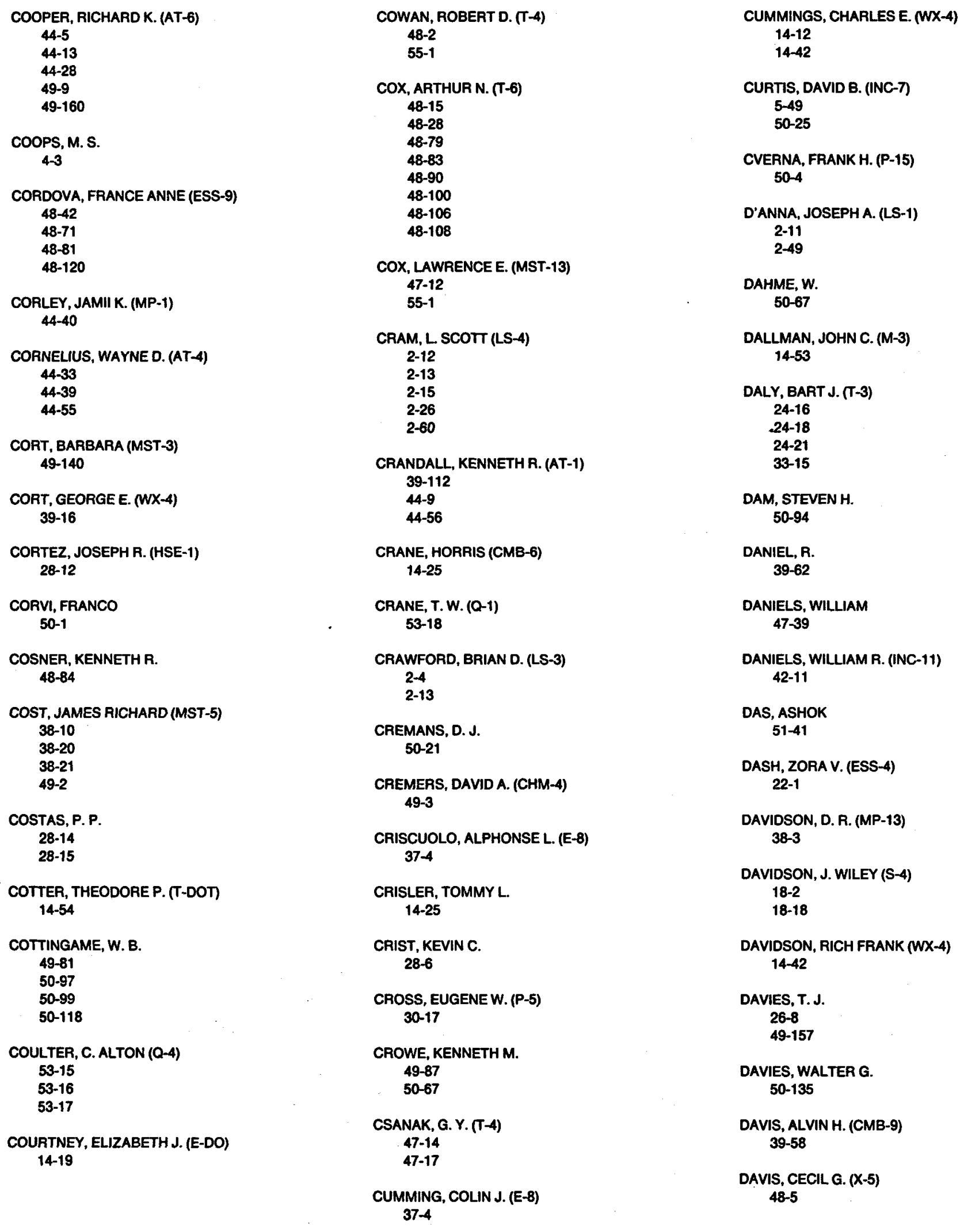


DAVIS, JOHN F., III (Q-14) 50-99

DAVIS, M. (MP-7) 49-130

DAVIS, MICHAEL J. 5-9 49-121

DAVIS, WILLIAM C. (M-3) 49-100

DAWSON, J. M. 30-18

DAWSON, W. K. (MP-1) 39-65

DAY, ROBERT H. (P-14) 14-49

31.41

49-106

DAYEM, HASSAN ALY (Q-4)

53-14

53-39

DAYTON, J. 49-130

DE FIELD, JAMES D. (HSE-5) 28-5

DE GASPERI, KATHLEEN D. (H-1) 39-49

DE GRAND, T. 51-36

DE LEON, NELSON (T-12)

47-69

49-121

DE LLANO, M.

49-10

DE POORTER, GERALD L. (LS-6) 16-40

42-5

42-6

42-24

DE VAULT, GUILLAUME P. (Q-7) 36-1

DE VRIES, RALPH M. (P-7) $50-5$

DEAN, JOHN W. (CTR-9) $37-4$

$37-13$

$37-15$

DECKER, L 53-28
DEHNHARD, DIETRICH

50-10

50-59

50-116

DEMBO, MICAH (T-10)

$2-6$

$2-9$

DEMUTH, NELSON S., JR. (Q-7) 33-14

DENBOER, M.L. 47-71

DENDY, JOEL E., JR. (T-7) 39-1

DENNIS, BERT R. (ESS-6) 21-6

DEPOORTER, G. L. 16-10

DERBYSHIRE, $M$. 5-7

DEROUIN, CHARLES R. (E-11) 11-3

DESJARDIN, ROBERT (E-5) 25-23

DESPAIN, KEITH HOWARD (X-2) 48-84

48-100

48-107

DETCH, J. LEWIS, JR. 31-26

DEUPREE, ROBERT G. (ESS-5) 39-58

48-116

48-117

DEUTSCH, J. 50-67

DEVANEY, JOSEPH J. (X-6) 49-15

49-111 50-103

DEVANEY, MARJORIE A. (C-8) 39-78

DEWITT, RICHARD J. (WX-11) $39-2$

DI PIPPO, R 49-154

DICK, JERRY JOEL (M-3)

$$
\text { 3-3 }
$$$$
\text { 3-4 }
$$

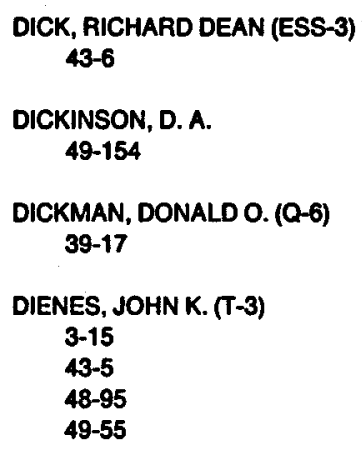

DIGIACOMO, NICHOLAS J. (P-3) 50-5

DINEGAR, ROBERT H. (M-7) 3-7

3-9

DISCH, RAYMOND L 47-44

DITZLER, W. R. 49-81

DIVEN, BENJAMIN C. (P-DO) 49-113

DIXIT, S. N.

47-11

47-16

47-27

49-35

DIXON, RAY D. (MST-6)
$14-25$

14-33

DOBRATZ, BRIGITTA (M-1) 26-6

DODDS, STANLEY A. 38-11 38-29

DOLL, JIMMIE D. (CHM-2)

5-13

5-45

5-72

5-78

5-81

39-71

39-72

DOLL, MARGARET A. (C-3) 39-29

DOMANY, EYTAN

49-27

49-28

DONAHUE, D. K. 2-49 
DONAHUE, JOEY B. (MP-7) 49-130

DONNERT, H. J. (MP-DO) 31-8

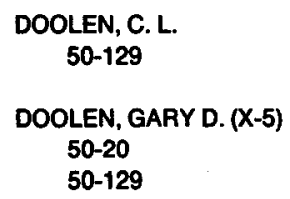

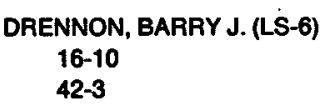

DROLSHAGEN, GERHARD (T-12) 47-26 47-70

DROPESKY, BRUCE J. (CNC-11) 50-37

DROPESKY, BRUCE J. (INC-11) 50-113

DRUHL, K. 5-80

DUBOIS, DONALD F. (T-11) 49-144

DUCHANE, DAVID V. (MST-6) 14-11

DUCHANE, DAVID V. (MST-7)

$$
\text { 14-46 }
$$

DUDZIAK, DONALD J. (S-4) 18-18

$30-2$

30-20

DUFFY, CLARENCE J. (INC-11) 16-14

48-69

DUKE, MICHAEL B. 48-96

DUKOWICZ, JOHN K. (T-3) 39-20

39-102 49-23

DUNBAR, DOUGLAS L. (P-14) 31-20

DUNCAN, D. R. 53-43

DUNCAN, LEWIS M. (ESS-7) 48-99 48-104

DUONG-VAN, MINH 51-34

DUPRE, ROBERT $R$. 48-40

DUPZYK, RONALD J. 50-46

DVORAK, ROBERT F. (HSE-1) 53-33

DYER, PEGGY (P-3) 50-135

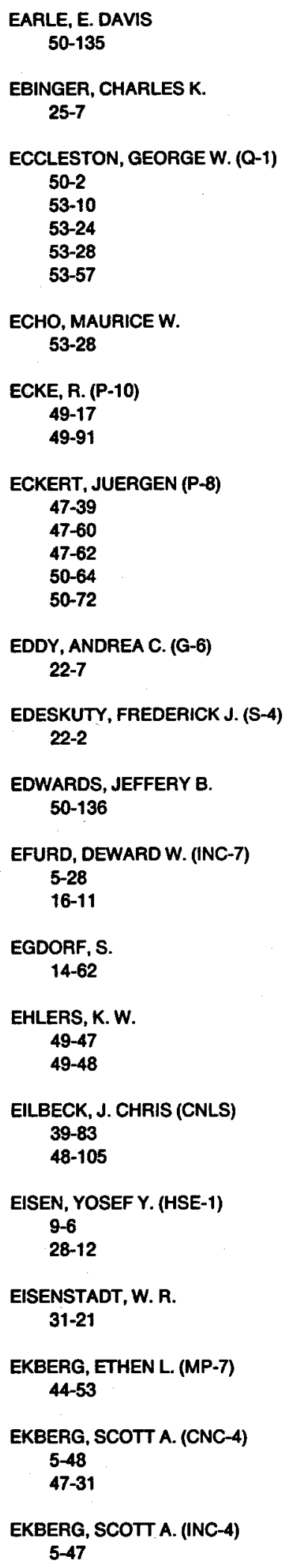


EKDAML, CARL A., JR. (P-1) 14-8

ELAM, WEST T. 49-24

49-147

ELDERS, WILFRED A

21-1

ELLER, P. GARY (INC-4) 5-40

ELLIOTT, C. JAMES $(X-1)$ 49-97

ELLIOTT, GUY R. B. (G-9) $31-2$

ELUOTT, REED O. (CMB-5) 38-20

ELLIS, WALTON P. (CHM-2) 5.73

47-12

55-1

EMBRECHTS, MARK J. (CTR-12) 18-2

18-11

EMBURY, JANONF. 49-88

EMELITY, LUDGARD A. (HSE-7) 42-13

ENGELKE, RAYP. (M-3) 47-66

ENGER, M. DUANE (LS-DO) 2-2 $2-39$

ENGER, M. DUANE (LS-3) 2-19

ENGLAND, TALMADGE R. (T-2) 50-123

ENGLEMAN, ROLF, JR. (CHM-1) 5-75

5-86

ENGLERT, $P$. 48-54

ENRIQUEZ, RAUL PEREZ 24-11

ENVIRONMENTAL SURVEILLANCE GROUP (H-8) 28-9

EPSTEIN, RICHARD I. (ESS-9)

48-44

48-47

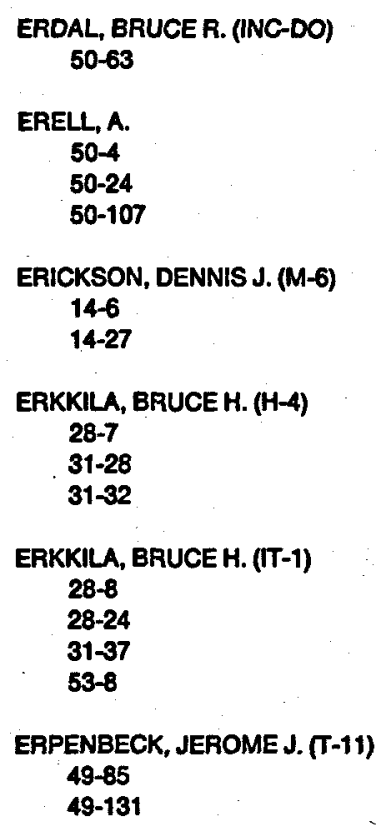

EVANS, W. DOYLE(ESS-9) 48-51

48-123

48-124

EWALD, ROBERT H. (C-DO) 39-18

EXTERMANN, RICHARD C. (P-3) 50-80

FABER, VANCE (C-3) 39-77

FAEHL, R. J. (X-8) 39-64 44-29

44-57

49-135

FAIRES, LYNDA M. (CHM-1) 5-75

47-32

FALLIEROS, $S$. 50-26

FALLIEROS, STAVROS L. $50-42$

FARKHONDEH, $M$. 51-45

FARNUM, DAVID J. (MP-13)

37-11

$38-45$

FARNUM, EUGENE H. (CMB-10) 14-45

FARRAR, CHARLES R. (Q-13) 48-92

FARRELL, JOHN ALDEN (AT-2) 44-9

44-41

FAUCET, JOHN A

50-69

$50-70$

FAUCHER, PAUL 48-2

FAW, R.E 50-71

FAZIO, MICHAEL V. (AT-5) 44-20

FEHLAU, PAUL E. (Q-2)

53-30

53-31

63-33

53-47

53-51

53-52 
FEINBERG, J.

17-19

FELDMAN, WILLIAM C. (ESS-8)
48-17

$$
\text { 48-17 }
$$

48-21

48-25

48-31

48-32

48-33

48-37

48-38

48-41

48-88

48-91

48-109

48-111

FELTHAUSER, HARRY E. (P-4) 48-2

FENIMORE, EDWARD E. (ESS-9)

31-6

48-2

48-55

48-66

48-123

48-124

53-53

FERGERSON, RAYMOND W. (MP-10) 49-26

FERGUSON, R. L.

50-45

50-84

FESSER, KLAUS (T-11)

51-15

FICK, D.

50-32

FICKETT, JAMES WILDON (T-10) 39-40

FICKETT, WILDON (M-3)

3-13

FIELDS, L.

53-42

FIFE, PAUL C.

39-42

$39-43$

FIGUEIRA, JOSEPH F. (AP-5) 32-2

FINK, CONRAD F. (G-4) 31-1

FINN, J. 50-51

50-86

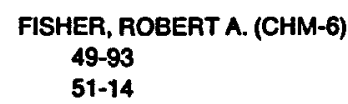

FISK, ZACHARY (MST-5)

38-18

38-19

38-23

38-31

38-32

38-37

$38-41$

$38-46$

$38-49$

49-87

49-103

FITZGERALD, THOMAS JOSEPH
$48-40$

FITZPATRICK, JOHN R. (MST-11) 5-38

FLEMING, DONALDG. 38-30

FLUAER, RANDY L. 31-30

FLY, GERALD $W .(0-13)$ 24-1

FOLEY, JOHN E. (Q-1) 53-10

FOLLANSBEE, PAUL S. (MST-5) 14-22 38-47

FOLTYN, STEPHEN R. (AP-2) 30-14

$32-4$

FOLTYN, STEPHEN R. (CHM-5) 49-119

49-143

FORD, GEORGE P. (CNC-11) 50-63

FORD, WENDELL (Q-4) 39-47

FOREHAND, HARRY M., JR. (Q-2) 9-2

$9-3$

9-7

FORSLUND, DAVID W. (X-1)

30-18

30-19

48-12

$48-30$

48-56

49-92
FORTUNE, H. T.

50-97

50-116

FOSTER, D. GRAHAM, JR. (T-2) 37-1

FOSTER, GEORGE $R$.

16-10

FOSTER, GEORGE R. (LS-6) 42-6

FOURNEY, WILLIAM L. 43-6

FOWLER, CLARENCE M. (M-6)

14-12

14-27

$14-37$

FOWLER, ERIC B. (LS-6)

42-10

FOWLER, J. D. (MST-5) 37-8

FOWLER, MALCOLM M. (INC-7) 50-45

50-84

50-105

FOX, WILLE. $(W X-4)$ 14-42

FOXX, TERALENE S. (H-8) 16-21

FOXX, TERALENE S. (HSE-8) 16-38

FOXX, TERALENE S. (LS-6) 42-22

42-23

FRAENKEL, ZEEV (P-3) 50-101

FRANCE, STEPHEN W. (Q-2) 53-34

FRANCZAK, BERNHARD J. 44-67

FRANEY, M. A.

50-10

50-59

50-115

FRANK, JAMES S. (MP-4) 49-18 $51-34$

FRANKE, PAUL R., JR. (ESS-DOT) 22-4

FRANKLIN, MARK 39-46 
FRANKO, A. J.

2-28

FRANKS, L. A.

26-8

49-157

53-3

53-23

FRANTZ, CHARLES E. (MST-5) 14-22

FRASER, JOHN S. (AT-7)

44-14

44-15

44-28

44-65

49-72

FRED, MARK

4-1

FREDERICKSON, PAUL O. (C-3) 39-90

FREEDMAN, BARRY A.

51-10

$51-29$

FREEMAN, DAVID L. (CHM-2)

39-71

39-72

FREEMAN, W. $\mathbf{S}$.

$51-45$

FREYER, JAMES P. (LS-1)

2-14

2-28

FRIAR, JAMES L. (T-5)

50-26

50-27

50-28

50-42

50-91

50-114

FRITZ, G.

48-123

48-124

FRITZ, GEORGIA T. (M-1) 5-60

FRITZ, JOSEPH N. (M-6) 49-41

49-43

49-124

FRITZ, T. A. (ESS-8) 48-53

FRY, HERBERT A. (INC-4)

5-29

47-38

47-63
FUKUMOTO, SADAYOSHI 44-39

FUKUSHIMA, EIICHI (INC-4) 2-51

FULLER, WENDY WEBB 49-67

49-128

FUNSTEN, $H$. 50-69

GABLE, CARL 16-33

GAC, FRANK D. (MST-6) 6-1 38-5

GALBRAITH, HAROLD W. (T-12) 47-6

$49-96$

GALLEGOS, ANTHONY F. (H-8) 28-16

GALLEGOS, JOSE D. F. (MP-8) 44-26

GALLIMORE, DAVID L. (CHM-1) 5-10 5-11

GALLUZZI, $F$. 47-57

GANCARZ, ALEXANDER J. (CNC-11) 50-53

GANDRUD, B. W. 16-23

GARCIA, CLEMENTE (Q-2)

53-5

53-47

GARCIA, SAMMY R. (INC-5) 5-22

24-4

GARDE, RAYMOND (HSE-7) 42-13

GARDNER, JAMIE N. (ESS-2) 22-6

GARMIRE, G.P. 48-81

GARRETT, B. C.

5-83

GARROTT, R. A. (LS-6) 16-12
GARY, S. PETER (ESS-8)

48-1

48-20

48-30

48-34

48-56

48-64

48-68

GaVATHAS, E. 50-130

$\underset{50-45}{\text { GAVRON, AVIGDOR I. (P-3) }}$ 50-84

GAZZALY, MAGDI

50-15 50-59

GEAGA, J. 50-15

GEESAMAN, D.F. 51-45

GEISIK, CARL (AT-2) 39-53

GENTRY, RICHARD A. (T-3) 49-157

GEORGE, JOHN S. (LS-DO) 2-36

GEORGE, TIMOTHY G. (MST-5)

40-3

$40-4$

$40-5$

$40-6$

GERRITY, DANIEL P. (CHM-2) 5-24

GERSTL, S. A. W. (T-DOT)

39-4

49-88

49-153

GIBBS, WILLIAM R. (T-5)

$47-3$

50-6

$50-57$

$50-68$

50-127

GIBSON, BENJAMIN F. (T-5)

50-28

50-33

50-85

60-91

GIBSON, E.F. 50-12

GIBSON, TED O. (AT-7) 49-50 


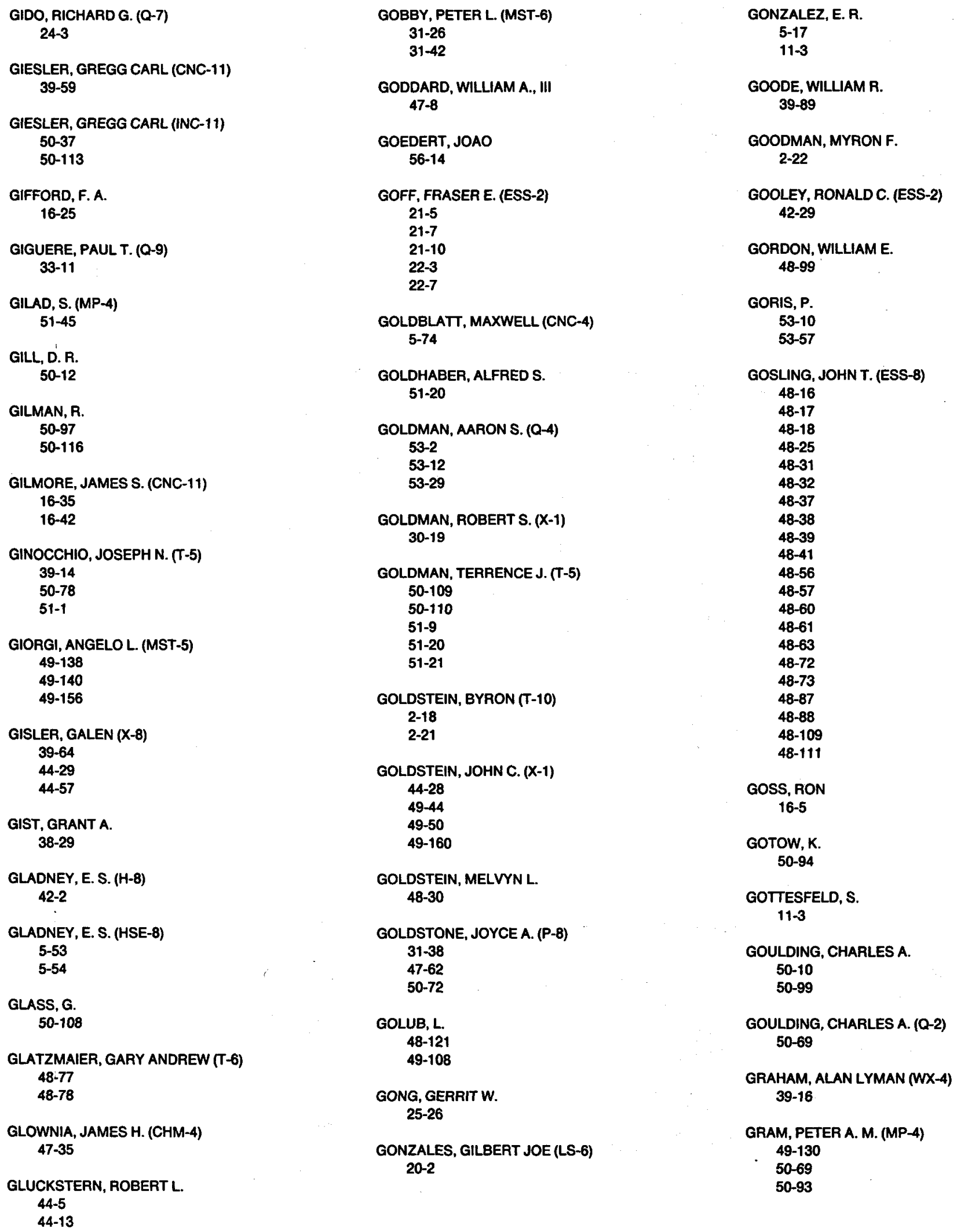


GRANDJOUAN, $N$.
$30-6$

GRATTON, E.

47-57

GREEN, J. L

48-74

GREENE, ARTHUR E. (X-2)

$14-64$

GREENE, S. J.

49-81

50-98

50-99

GREENE, S. J. (MP-10)

50-97

GREENWOOD, L. R.

38-3

GREGQ, CHARLES T. (LS-DO)

2-31

2-52

GREGOIRE, GHISLAIN

50-67

GREGORY, WILLIAM S. (Q-6)

24-7

39-116

GRIBBLE, ROBERT F. (CTR-4)

$17-2$

$17-6$

$17-20$

17-24

GRIEGO, RICHARD

2-18

GRIFFIN, JAMES HARPER (C-10) 39-67

GRIFFITH, JEFFREY KNOWLES (LS-3) 2-13

GRIMM, EUZABETH A.

2-37

GRISHAM, DONALDL (MP-7)

$44-3$

44-7

44-22

44-50

44-52

44-53

44-54

GROSNICK, D.

49-18

51-34

GROSS, E. E.

50-94
GRUEBLER, WILLI (P-DOR) 50-32

GSCHNEIDNER, KARL A. 49-147

GSCHNEIDNER, KARL A. 49-24

GUBERNATIS, JAMESE. (T-11) 49-27

49-28

49-141.

GUPTA, LAXMI C.

38-11

$47-37$

GURALNIK, GERALD D. (T-DO) 51-26

GURLEY, LAWRENCE R. (LS-1) 2-49

GURSKY, JUDITH C. (P-3) 50-131

GUSTAFSON, R. A. 39-108

GUTIERREZ, $G$.

49-10

GUTIERREZ, JOSE W. (ESS-11) 31-34

GUTMACHER, R. G. (Q-4)

4-1

53-15

53-16

53-43

GUTSCHICK, VINCENT P. (LS-6) 2-58

$39-92$

HAFF, K.W.

53-44

HAGENSON, RANDY L.

17-22

18-2

18-11

HAIRE, RICHARD G.

38-16

HAKKILA, E. ARNOLD (Q-4)

53-14

53-39

HAKONSON, THOMAS E. (LS-6)

16-30

42-2

42-6

42-24
HALBIG, JAMES K. (Q-1)

53-27

53-54

hale, gerald M. (T-2) 50-20

50-87

HALLECK, MARGARET S. (LS-1) 2-44

HALLECK, PHILLIP M. (ESS-3) 45-1

HALLIDAY, KAREN R. 6-37

HALLIN, AKSEL (MP-4) 49-18

HALLIN, AKSEL L. (MP-4) 51-34

HAMEROFF, STUART R.

$$
\text { 2-10 }
$$

2-43

HAMM, MARIANNE E. (Q-2)

9-8

9-10

14-17

50-69

53-22

53-56

HAMMEL, BRUCE A. (P-1) 49-30

HAMMEL, JAY E. (CTR-DO) 17-12

HAMMER, CHARLES F. (CTR-4) 18-3

HAMMOND, C. F. (CHM-1) 5-2

HAMMOND, ROBERT B. (E-11) 14-26

14.30

14-31

14-52

14-68

$31-21$

31-29

31-35

38-13

HANER, J. M. $37-4$

HANOLD, ROBERT J. (ESS-DOT) 22-3

27-2

27-3

HANRATTY, T.J. 


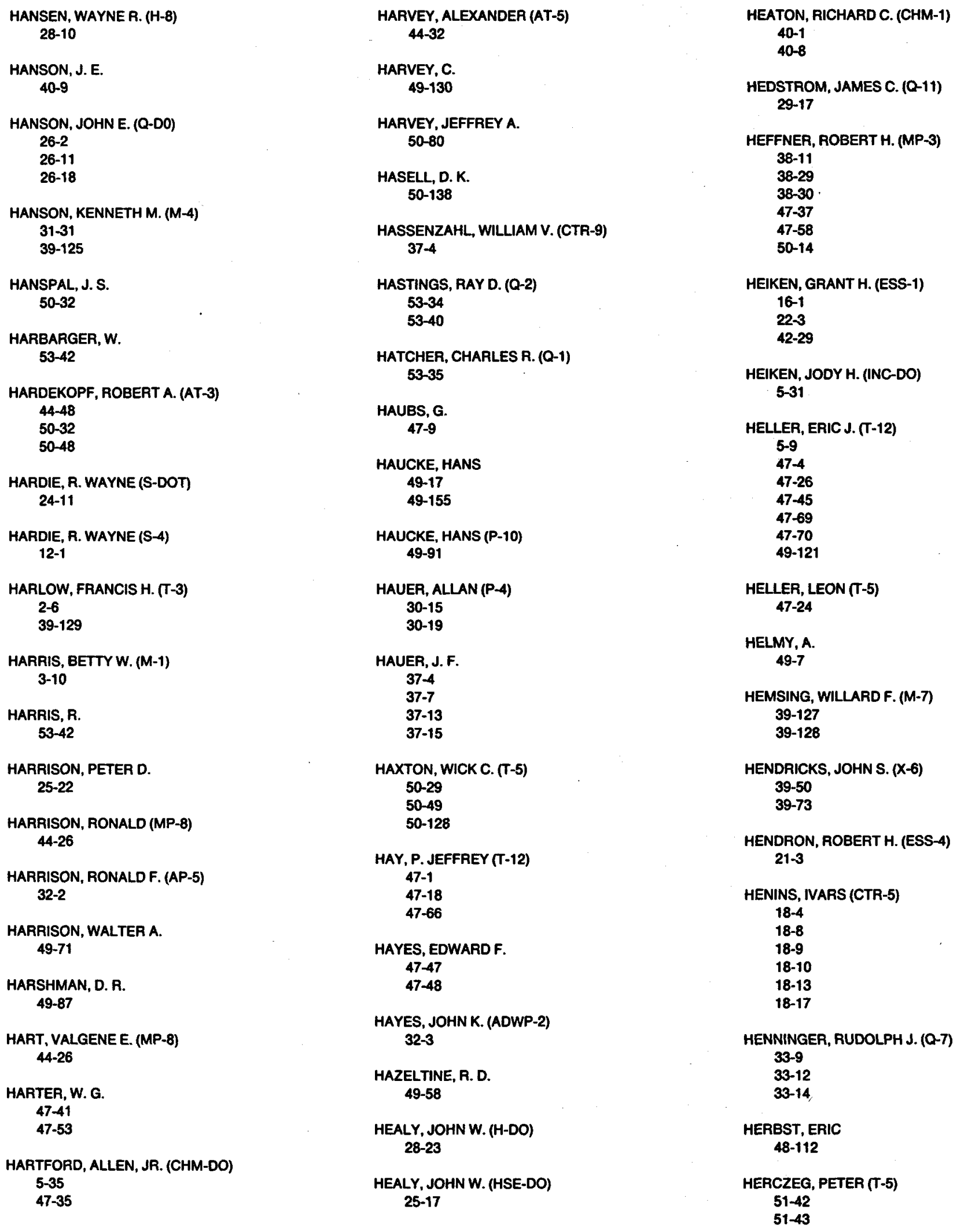




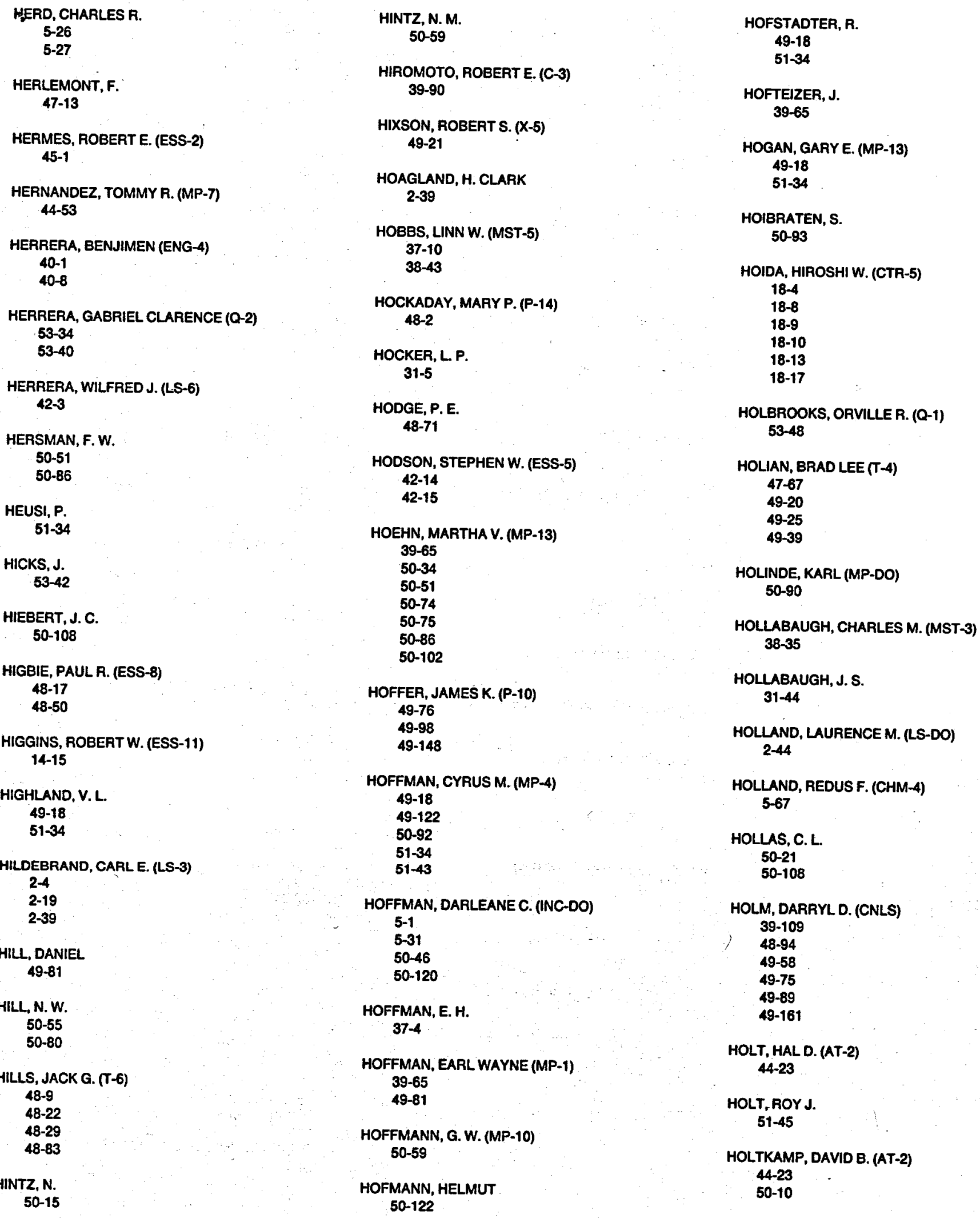




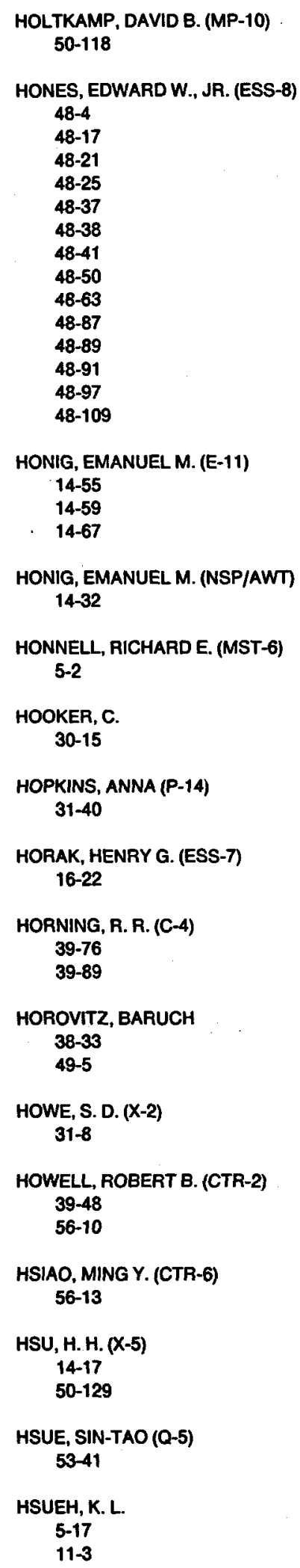

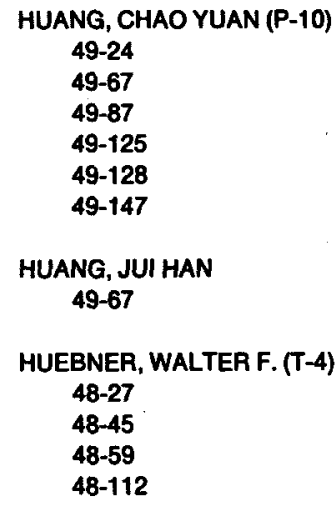

HURLEY, GEORGE F. (MST-5) 37-10 $38-9$

HUTSON, JUDITH C (INC-4) $2-7$

HUTSON, RICHARD L. (MP-3) 38-11

HYDE-WRIGHT, C $50-51$

50-86

HYDE, P. J. (E-11)

$11-3$
HYMAN, JAMES M. (T-7) 39-36

$39-79$

$39-80$

HYNES, M. V. (MP-4) 50-11

HYNES, M. V. (P-15) 50-94

IDAR, E.S. (Q-6)

24-3

IDZOREK, G. C. (MP-10) 28-17 50-10

IGARASHI, Z. 44-39

IGO, G. 50-15

IKEGAMI, KIYOSHI 44-39

IMAI, K. 49-81

IMAMURA, JAMES N. (ESS-9) 48-11 48-13

INAL, OSMAN T. $37-11$

38-45

INGRAHAM, JOHN C. (CTR-2) 17-8

INGRAM, $Q$. 50-24 50-107

IPAVICH, FRED $M$. 48-18

IRELAND, JOHN R. (Q-7) 33-14

33-21

33-23

IROM, FAROKH

50-24

50-59

50-107

IVERSEN, STEVEN 50-19

IVERSON, STEVEN G. 31-16

JACKSON, DARRYL D. (CHM-1) 5-12 


\begin{tabular}{|c|c|c|}
\hline \multirow{4}{*}{$\begin{array}{l}\text { JACKSON, JAMES O. (HSE-5) } \\
28-14 \\
28-15 \\
\text { JACKSON, JASPER A., JR. (ESS-3) } \\
45-2\end{array}$} & $\begin{array}{l}\text { JENNINGS, LEROY } \\
31-16\end{array}$ & $\begin{array}{l}\text { JONES, CLAUDE R. (CHM-6) } \\
49-100\end{array}$ \\
\hline & JENSEN, REED J. (P-DO) & JONES, D. R. TIMOTHY \\
\hline & $32-3$ & $51-48$ \\
\hline & JETER, I. W. & JONES, ERIC M. (ESS-5) \\
\hline JACKSON, PAUL J. (LS-4) & $53-46$ & $16-18$ \\
\hline 2-13 & & \\
\hline $2-54$ & $\begin{array}{c}\text { JET, JAMES H. (LS-4) } \\
2-3\end{array}$ & $\begin{array}{r}\text { JONES, K. } \\
50-15\end{array}$ \\
\hline JACKSON, SYDNEY V. (S-4) & 2-13 & \\
\hline $12-1$ & $5-34$ & JONES, K. W. (MP-10) \\
\hline & $47-46$ & $50-31$ \\
\hline JACOBSON, ABRAM R. (CTR-2) & & $50-62$ \\
\hline $17-1$ & JOHANSON, WILLIAM R. (MST-5) & \\
\hline $17-16$ & $49-73$ & JONES, LARRY A. (P-1) \\
\hline 17-18 & & $14-21$ \\
\hline $17-23$ & JOHANSSON, BORJE & 14-23 \\
\hline $17-25$ & 48-75 & $17-5$ \\
\hline $17-32$ & & \\
\hline $17-33$ & JOHNSON, JAMES D. (T-4) & JONES, LARRY A. (P-6) \\
\hline $56-3$ & $\begin{array}{l}47-64 \\
47-67\end{array}$ & 49-30 \\
\hline JAHAN, M. SHAH & $49-20$ & JONES, LLEWELLYN H. (INC-4) \\
\hline $50-130$ & $\begin{array}{l}49-25 \\
49-39\end{array}$ & $\begin{array}{r}5-29 \\
5-47\end{array}$ \\
\hline JAHODA, FRANZ C. (CTR-8) & $49-61$ & $5-48$ \\
\hline $17-4$ & $49-70$ & 5-55 \\
\hline $17-19$ & & 47-31 \\
\hline $17-37$ & JOHNSON, KAYE ALLAN (MST-11) & 47-38 \\
\hline & $30-3$ & $47-40$ \\
\hline JAMESON, ROBERT A. (AT-DO) & $38-50$ & \\
\hline 44-1 & & JONES, MARIANNE M. (CHM-1) \\
\hline 44-2 & JOHNSON, KAYE ALLAN (MST-13) & 8-1 \\
\hline 44-24 & $5-23$ & \\
\hline 44-34 & $38-8$ & JONES, MICHAEL E. $(X-8)$ \\
\hline $44-35$ & 38-26 & 44-25 \\
\hline 44-55 & & $49-49$ \\
\hline & JOHNSON, M. W. (AT-5) & 49-57 \\
\hline JANSSEN, ROBERT D. (CNLS) & $50-131$ & $49-136$ \\
\hline $\begin{array}{l}39-22 \\
49-126\end{array}$ & JOHNSON, MARK E. (S-1) & 49-139 \\
\hline & $39-57$ & JONES, ROBERT W. (Q-11) \\
\hline JARAMILLO, STEVENA. (P-15) & & $29-3$ \\
\hline $31-20$ & JOHNSON, MIKKEL B. (MP-DO) & 29-4 \\
\hline $31-26$ & $50-22$ & 29-7 \\
\hline $31-42$ & $\begin{array}{l}50-23 \\
50-89\end{array}$ & $\begin{array}{l}29-11 \\
29-15\end{array}$ \\
\hline JARBOE, THOMASR. (CTR-5) & $50-90$ & \\
\hline $18-4$ & $50-96$ & JONES, ROGER D. $(X-1)$ \\
\hline $\begin{array}{l}18-8 \\
18-9\end{array}$ & $50-112$ & $30-13$ \\
\hline $18-10$ & JOHNSON, NORMAN LEE (WX-4) & JONES, $\mathbf{S}$. \\
\hline $18-13$ & $39-16$ & $53-42$ \\
\hline $18-17$ & & \\
\hline & JOHNSON, SUSAN S. (Q-1) & JONES, WESLEY M. (CHM-2) \\
\hline $\begin{array}{l}\text { JARMER, JOHN J. (MP-7) } \\
\text { 49-81 }\end{array}$ & 53-28 & $\begin{array}{l}5-21 \\
5-85\end{array}$ \\
\hline & JOHNSTONE, J. KEITH & \\
\hline JARMIE, NELSON (P-3). & $26-3$ & JORDAN, THOMAS LEE (C-3) \\
\hline $50-48$ & & $39-19$ \\
\hline JABYINEN GOBDON D INC-4) & $\underset{30-14}{\text { JOLIN, L. J. (AP-2) }}$ & $\begin{array}{l}39-90 \\
39-107\end{array}$ \\
\hline $\begin{array}{l}\text { JAFIVINEN, GORDON D. (INC-4) } \\
\text { 5-58 }\end{array}$ & $32-4$ & ovitis : \\
\hline JENKS, RICHARD P. (Q-7) & JOLIN, L. J. (CHM-5) & $\begin{array}{l}\text { JORDAN, THOMAS M. } \\
\qquad 49-86\end{array}$ \\
\hline $33-9$ & $49-119$ & \\
\hline
\end{tabular}




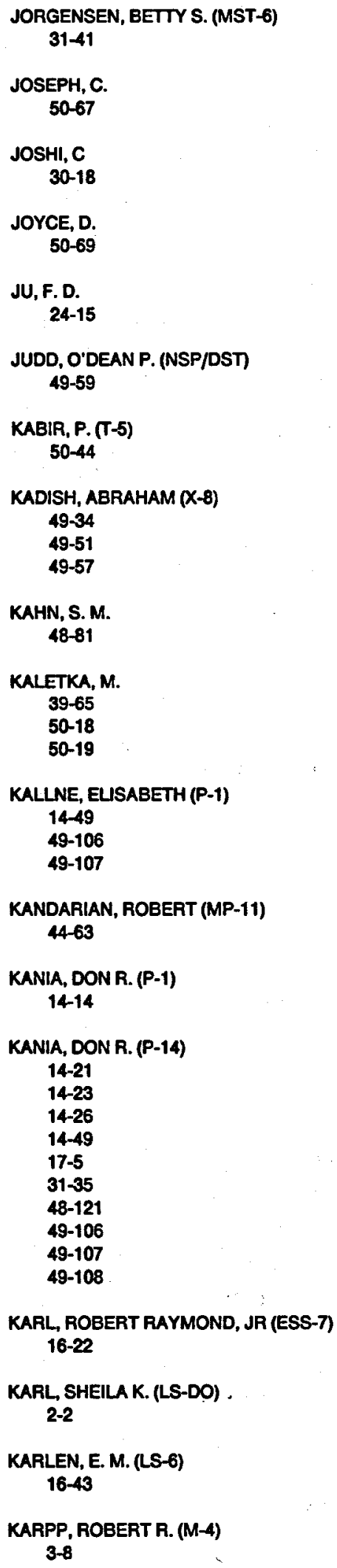

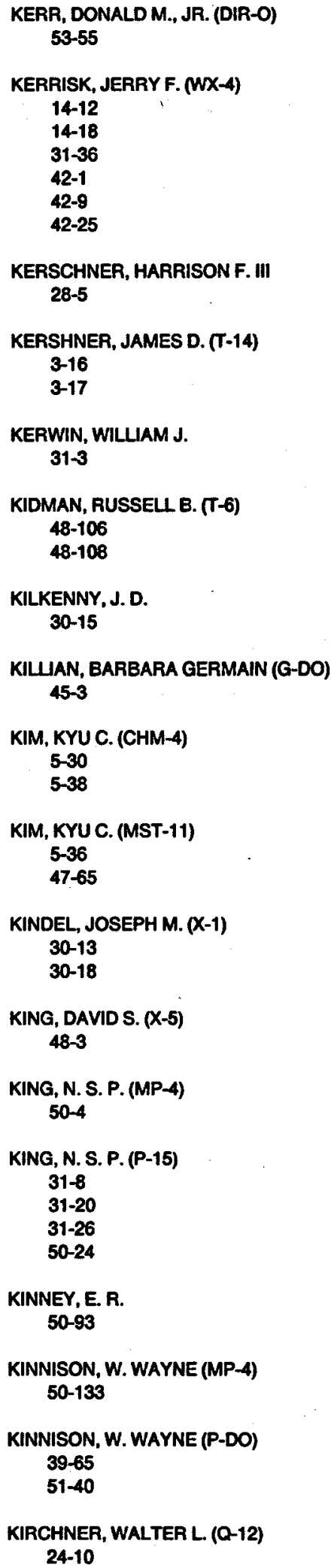


KIRKPATRICK, RONALD C. (X-2) 48-98

KIZIAH, R.R. 49-110

50-97

50-98

KLEBESADEL, RAY W. (ESS-9) 48-55

48-66

48-123

48-124

KLEIER, DANIEL A. (T-12)

47-66

KLEIN, AMIR

50-89

50-125

KLEIN, RICHARD I.

48-93

KLEINSCHMIDT, PHILLIP D. (MST-5) 38-16

KLINGNER, PHILLIP L. (CTR-5) $17-36$

18-10

KLOET, WILLEM M. 50-79

KLOSTERBUER, SHIRLEY F. (Q-1) 53-27

KNAB, DARYL (H-8)

5-54

KNAPP, ROGER D.

47-5

KNIGHT, JERE D. (NC-11)

16-35

$16-42$

KNIGHT, THAD D. (Q-9)

24-19

33-24

KNOBELOCH, GORDON W. (INC-11) 5-28

16-11

KNOWLES, HARROLD B. (MP-3) 44-66

KNOX, STEPHEN O. (CTR-5) 18-9

18-10

KOBASHI, K

49-7

49-114

49-115
KOCIMSKI, S. M.

53-3

53-23

KOCKS, U. FRED (CMS)

38-36

38-47

KOCZAN, STEVEN P. (ESS-6)

14-60

KOENIG, RALPH A., JR. (H-7) 42-12

KOESTEL, A.

$$
\text { 24-3 }
$$

KOLB, EDWARD W., JR. (T-6) 48-24

KOUE, J.J.

16-20

KOLSTAD, CHARLES D. 25-11

KOPP, ROGER ALAN (X-1) 48-43

KORDESCH, K. V.

$11-3$

KOSIEWICZ, CLAUDIA E. (Q-13) 29-6

KOSIEWICZ, STANLEY T. (CHM-1) 40-8

KOSTELECKY, VACLAV ALAN (T-8) 39-111 47-22

KOVASH, M. A. 50-94

KOZLOWSKI, THOMAS (MP-10) 39-65

KRAEMER, PAUL M. (LS-4)

2-12

2-26

2-60

KRAKOWSKI, ROBERT A. (CTR-12) 17-8

18-2

18-11

18-15

37-5

KRANE, KENNETH S. 50-69

KRANZ, ROBERT

$$
\text { 42-16 }
$$

KRAUSHAAR, J.J. 50-12 $\underset{5-66}{\text { KRESSIN, NAN K. (H-5) }}$

KRICK, MERLYN S. (Q-1) 53-10

KRIMIGIS, S. M. 48-35

KRISTAL, RICHARD (P-4) 30-19

KRISTIAN, JEROME 48-101

KROHN, BURTON J. (T-12) 47-23

KRUMLINDE, J. 50-8

KRUPKA, MILTON C. (S-4) 11-1

KRUSE, HERALD W. (P-14) 31-5

KUBAS, GREGORY J. (INC-4) 5-59

47-7

KUCKERTZ, THOMAS H. (E-B) 53-34

53-53

KUMAR, PRADEEP 51-35

KUNG, H. T. $39-46$

KUNKLE, THOMAS D. (ESS-5) 39-58

KUNZ, JUTTA (T-8)

50-50

50-56

50-88

KUNZ, WALTER E. (Q-2)

14-3

53-23

53-34

53-40

53-44

KUPERSHMIDT, BORIS ABRAM (CNLS) 48-94

$49-75$

$49-89$

49-161

KURNIT, NOAMAN A. (CHM-6)

14-51

49-9 


KWAN, THOMAS J. T. $(X-8)$
$39-88$
$49-52$
$49-60$
$49-84$
$49-120$

KYLE, GARY S.

50-59

LABAUVE, RAPHAEL J. (T-2) 18-2

LACOE, RONALD C.
49-24 49-24

49-128

49-147

LADELFE, CAROL M. (ESS-3) 21-8

LADISH, JOSEPH S. (P-14) 31-16

LAGUNA, GLENN A. (CHM-4) 5-65

LAMBERT, JAMESE. (MP-7) 44-3

44-22

44-50

44-52

44-53

44-54

LAMBERT, STEVEN E. 49-87

LANE, LEONARD J. (LS-6)

16-30

42-27

42-30

LANGHORST, GARY J. (LS-6)

20-1

42-5

LAPEDES, ALAN S. (T-6) 50-40

LAQUER, HENRY L. (Q-10) 11-1

LARIMER, R. M. 50-3

LAROS, JOHN G. (ESS-9)

48-55

48-66

48-123

48-124

LARSEN, EDWARD W. (T-1) 39-32
LARSEN, EDWARD W. $(x-6)$

39-62

$39-63$

39-82

39-105

LARSON, ALLEN C. (INC-4) 5-82

LATHROP, BRAXTON L. (C-3) 39-8

39-41

LATHROP, JUDITH M. (IS DO) 49-146

LAUGHUIN, A. WILLIAM (ESS-2) 16-24

LAURINAT, J. E. 14-53

LAWRENCE, J. $M$.

38-37

$38-49$

47-71

LAWRENCE, JAMES N. P. (H-1) 28-28

LAWRIE, DUNCAN $39-46$

LAWSON, A. C. (MST-5) 49-156

LAWSON, ROBERT E., JR. 16-41

LAYNE, SCOTTP. (CNLS) 2-38 2-47

2-56

LAZARUS, GLORIA S. (Q-11) 29-7

LAZRUS, ALLAN L. 16-23

LEAL, EMERSON P. 47-17

LEBEDA, CHARLES F. 48-20 48-40

LEBRUN, M. $50-67$

LEE, D. D. 48-80

LEE, DAVID 50-105
LEE, HUAN $(X-8)$

44-25

49-49

49-136

49-139

LEE, JAMES T., JR. (ESS-7) 16-41

LEE, KENNETH $(X-1)$ 48-12

48-56

LEE, KIEN Y (M-1) 5-25

LEE, M. A. 48-30

LEE, PETER H. Y. (P-1) 30-5

LEE, PING (P-4) 49-107

LEE, WEN HO (X-7) 49-6

LEEMANN, $B$. 50-3

LEHNERT, BRUCE E. (LS-1) 2-50

LEITCH, M. J. (MP-4)

50-4

50-94

LEMAIRE, J.

47-13

LEMONS, DON S. $(X-8)$

44-25

49-136

49-139

LENNARTSSON, $W$. 48-53

LENNON, GREGORY G. (T-10) 2-45

LEON, MELVIN (MP-3)

38-11

38-29

47-37

47-52

47-58

49-69

LESAR, RICHARD (P-10) 47-21 


\section{LESAR, RICHARD A (T-11) \\ 47-19 \\ 47-25}

LESTER, T. $W$. 50-71

LEUNG, CHUN MING 48-112

LEUNG, K. N. 49-47

49-48

LEVINGS, R. L.

14-10

LEVY, SCHON S. (ESS-1) 42-18

42-26

LEWIS, GARY K. (MST-6) 14-33

LEWIS, H. RALPH, JR. (CTR-6)

56-4

56-5

LEWIS, PAUL S. (E-B)

$39-30$

39-44

U. T. K. (a-1)

53-19

LI, Y. Y.

48-10

LIANOS, D.

50-130

LIAUD, P. 50-132

LIBERMAN, DAVID A. (T-4) 47-68

$49-95$

LCHTENSTADT, J. 60-93 50-107

LICTENSTADT, J.

50-24

LIEBENBERG, DONALD H. (P-10) 49-42

LIEBETRAU, $A$.

53-2

LIEPINS, RAIMOND (MST-6)

31-41

49-83

LILES, DENNIS R. (Q-9)

24-20

33-11

33-19
LIM, HWA-AUN

51-41

LIM, J. J.

53-7

LIME, JAMES F. (Q-7) 24-3

LINDAU, I.

55-1

LINDBERG, HELEN A. (INC-DO) 5-31

LINFORD, RULON K. (CTR-DO)

$17-36$

18-9

18-17

LINTON, THEODORE W. (CTR-4)

17-24

$17-35$

LSOWSKI, PAUL W. (P-3)

31-8

50-132

LITTLE, EDWARD M. (CTR-7) 17-28

UTTLEJOHN, GEORGE J. (HSE-1) 28-12

LU, LON CHANG (INC-11)

50-13

50-16

50-19

$50-65$

50-113

LOCKHART, MILTON GLENN (ADP-2) 39-11

LOCKYER, $N$.

50-92

LOGE, GARY WAYNE (CHM-4) 47-35

LOHE, MAX A.

39-108

LOMDAHL, PETER $S$.

51-15

LOMDAHL, PETER S. (CNLS)

2-42

2-56

2-61

38-33

39-12

48-105

49-5
LONDON, JERRY E. (LS-1)
$2-48$
$2-59$
$3-18$

LONDON, ROBERT E. (INC-4) 2-7 $2-29$

LONGMIRE, JERRY L. (LS-3) 2-4

LONGMORE, A. J. 48-82

LOONEY, LARRY D. (P-14)

14-9

14-57

31-15

$31-30$

$31-40$

LOREE, THOMAS R. (CHM-6) 2-17

LOUCK, JAMES D. (T-7) 39-108

LOUGHEED, R. W. 50-46

LOURIE, R. 50-51 50-86

LOVE, W. GARY 50-115

LOWE, LEWIS H. (WX-11) 39-98 39-103

LUBECK, OLAF M. (C-3) 39-52

LUCAS, MARCIA C. (Q-2) 9-8

9-10

14-17

53-5

53-56

LUCERO, J. P. (M-5) 31-39

LUDFORD, G. S. S. 49-126

LUEDEMANN, GARY (AT-5) 49-50

LUMPKIN, ALEX H. (P.DO)

44-14

49-64

49-72

LUO, HUEY LIN 49-128 
LYLE, ALVIN R. (INC-5) 24-9

LYNCH, MICHAEL T. (WX-7) 49-50

LYNN, DAVID K. (E-11) 11-2

LYONS, PETER B. (P-DO) $31-30$

LYONS, PETER B. (P-14) 14-57

31-16

$31-40$

LYSENKO, WALTER P. (AT-6) 39-97

MAARANEN, STEVEN A. (ADPA) 25-26

MAC ARTHUR, DUNCAN W. 49-130 $50-93$

MAC FARLANE, ROBERT E. (T-2)

37-1

39-115

$50-35$

50-111

MAC INNES, MARK A. (LS-3) 2-34

MAC LAUGHUN, DOUgLAS E. 38-11

$47-37$

47-58

$50-14$

MAC ROBERTS, MARTIN D. (E-DO) 14-19

MACEK, ROBERT J. (MP-13) 44-7

44-50

MACHADO, LUIZ E. 47-17

MACK, JOSEPH M., JR. (X-5) 31-16

MACK, JOSEPH M., JR. (X-6)

14-17

49-86

MACKEN, CATHERINE A. 2-32

$2-37$

$2-40$

MACLAUGHLIN, DOUGLASE 38-29

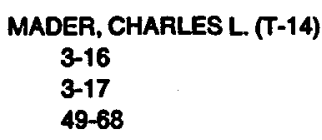

$50-106$

MADSEN, J.

48-44

MAEDER, P.F. 49-154

MAENO, YOSHITERU

$$
\text { 49-17 }
$$

49-91

49-155

MAES, MAXN. (HSE-8) 16-44

MAGEE, NORMAN H. $(T-4)$

48-27

48-59

MAGGIORE, CARL J. (E-11)

5-73

$11-3$

16-14

48-69

MAGNUSON, D. W.

49-157

MAGNUSON, JOHN E. (MST-6) 38-5

MAHAFFY, JOHN H. (Q-9) 33-19

MAIER, OTTO A. (MEC-3)

39-86

MAIER, WILLIAM B., II (CHM-4)

5-64

5-67

MANKZRAK, CHARLES F.

47-39

MAKO, F. 49-135

MAKOWITZ, HENRY 1731

MALENFANT, RICHARD E. (Q-2) 9-3

9-4

9-7

9-13

MALENFANT, RICHARD E. (S-4) 9-14

10-1
MALM, JOHN G

5-40

MALONE, ROBERT C. (CTR-6) 48-102

MAMPE, $W$. 50-132

MANGENG, CAROLYNA. (S-4) 52-2

MANLEY, JOHN H 50-61

MANN, FREDERICK $W$. 50-111

MANTEUFFEL, THOMAS (C-3) 39-77

MANTEUFFEL, THOMAS A. (C-3) 39-36

MAPLE, MERRIL B $38-24$ $49-87$

MARGOLIN, LEONARDG. (ESS-5) 16-4 $16-45$

MARIAM, FRANK (MP-13) 49-18

51-34

MARKIN, JACK T. (Q-4)

53-15

53-16

53-17

53-52

MARKLIN, GEORGE J. (CTR-6) 56-15

MARKS, THOMAS (MP-1) 53-41

MARMION, D. M. 5-60

MARR, DUANE R. $(X-6)$ 39-118 39-119 39-120

MARSDEN, JERROLDE. (CNLS) 49-5B

MARSH, G. L 16-41

MARSH, SINCLAIR F. (CHM-1) 5-51

MARSH, STANLEY P. (M-6) 14-12 
MARSHALL, B. R.

26-8

MARSHALL, J. A.

$50-76$

MARSHALL, ROBERT S. (Q-4)

53-25

53-29

MARSTERS, ROBERT G. (MST-6) 38-22

MARTENSSON, NILS

48-75

MARTIN, JOANNE L. (C-3) 39-23

MARTIN, JOHN C. (LS-4)

2-3

5-34

47-46

MARTIN, RICHARD A. (Q-DO) 39-37

MARTIN, RICHARD L. (T-12)

47-18

47-29

MARTINEZ, AMRON M. (HSE-5) 28-4

MARTINEZ, ELMO H. (LS-3) 2-44

MARTINEZ, JAKE (Q-2) 53-47

MARTINEZ, RONALD G. (C-3) 39-52

MARTOFF, C.J. 50-67

MARTZ, HARRY F., JR. (S-1)

24-12

24-17

$39-87$

MASON, K. O.

48-42

48-71

48-81

48-120

MASSEY, ROBERT S. (CTR-1) 17-10

MASTERSON, JEANNENE M. (M-4) 28-26

MASTERSON, T.G.

50-12

MATHEWS, MARK A. (ESS-3) 21-8
MATIS, H. S. (MP-4)

49-18

$50-24$

50-107

51-34

MATLACK, GEORGE M. (CHM-1)

5-57

38-16

40-1

40-8

MATLACK, GEORGE M. (CMB-1) $50-52$

MATSUNAGA, ISAO 214

MATTHEWS, J. L. 50-93

MATWIYOFF, NICHOLAS A. (INC-DO) 2-7

MAULDIN, G.H. 14-63

MAYER-KRESS, GOTTFRIED (CNLS) 47-9

MAYER, ANTON (MST-6) 14-13

MAYER, FREDERICK J. 14-48

MAYER, STUART 48-19

MAYNARD, ROBERT L. 14-48

MAZUMDAR, SUMIT (CNLS)

47-11

47-16

47-27

$49-35$

$51-36$

MC CABE, CHARLES W. (MP-3) 31-33

MC CALL, GENE H. (X-DO) 30-7

$30-9$

MC CARTHY, J.S. 50-99

MC CLARY, WILMA J. (IS-4) 26-12

MC CLELLAND, JOHN B. (MP-10) 50-15

50-59

50-62
MC CLURE, PETER R. (LS-6)

2-54

MC COMAS, DAVID J. (ESS-8)

$31-4$

48-17

48-21

48-37

48-38

48-41

48-87

48-109

MC CONNELL, JOHN R. (MP-12) 44-63

MC CORMICK, J. BYRON (E-DO) 31-3

MC CORMICK, J. BYRON (E-11) 11-2

MC CORMICK, ROBERT NORMAN (M-7) 14-7

MC COY, FLOYD J.

16-1

MC COY, R.W. 5-60

MC CRAY, MARK S. (P-10) 49-4

MC CULLOUGH, E. 53-46

MC DANIELS, DAVID K. 50-69

MC DONALD, ARTHUR BRUCE 50-135

MCELLIN, S. (LS-6) 20-1

MC FARLAND, LARRY C. (ISD-6) 25-19

MCFARLAND, ROBERT D. (Q-11)

29-4

29-7

$29-8$

29-15

MC GETCHIN, THOMAS A. 16-18

MC GILL, JOHN A.

50-59

MC GIRT, A. FRANK (C-9)

39-34

$39-45$

39-95

39-114

39-130

39-131 
$\underset{\text { MOFFET, D. }}{\text { MOF. }}$

MOINESTER, MURRAY A.

50-4

50-24

50-107

MOLLER, PETER

50-8

MONDT, JOHANNES P. (CTR-6) 56-14

MONTOYA, CONSUELO (LS-6)

20-1

MOODY, DAVID C. (CNC-4) 5-39

MOODY, DAVID C. (INC-4) 5-76

5-77

MOORE, C. FRED

50-97

$50-99$

50-116

MOORE, DAVID S. (CHM-4)

3-11

49-137

MOORE, J. A

24-6

MOORE, KURT R. (ESS-10)

$\mathbf{3 1} \mathbf{3 4}$

$39-130$

MOORE, MICHAEL S. (P-3)

50-1

$50-80$

MOORE, STANLEY W. (Q-13) 29-8

29-14

MOREL, J.E. (X-6) 39-48

MORENO, DAWN K. (CMB-10) 38-22

MORGADO, RICHARD E. (Q-2) 63-23

MORI, WARREN B. $(X-1)$ 30-18

MORI, YOSHIHARU 44-39

MOROSIN, BRUNO

$$
\text { 5-82 }
$$

MORRIS, CHARLES E. (M-6) 49-41

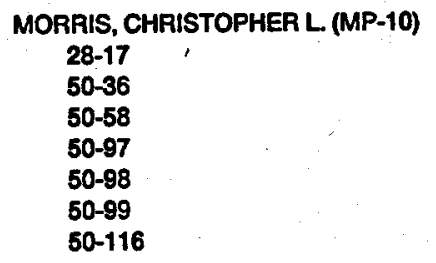

MORRISETT, JOEL D.

47-5

MORRISON, P.J.

49-58

MORROW, PAUL E

2-50

MOSES, JOHN D. (P-3)

$50-80$

50-132

$51-45$

MOSES, RONALD W., JR. (CTR-6)

17-10

17-18

$17-22$

$17-32$

$17-33$

$56-3$

MOSS, CALVINE. (Q-2)

9-8

9-10

14-17

$31-10$

53-5

53-22

53-53

53-56

MOSS, J. (MP-10) 50-15

MOSS, JOEL MARSHALL (P-2) $50-62$

MOSS, JOEL MARSHALL (P-3)

50-104

50-119

MOSTROM, MICHAEL A. (X-8) $49-120$

MOTTOLA, EMIL

$$
50-40
$$

MOUSSA, PIERRE

$39-39$

MROZ, EUGENE J. (INC-7)

$16-31$

\section{MUELLER, $M$ 30-19}

MUIR, DOUGLAS W. (T-2)

8-1

18-7

50-111

MULLER, MARCIA (H-B)

42-2

MULUNS, LAWRENCE J., JR. (MST-13) 5-52

MUNK, A. CHRISTINE (LS-3)

$2-4$

MURAMOTO, FRANK S. 21-1

MURDOCH, BRUCE T.

50-138

MURPHY, HUGH D. (ESS-4)

22-1

27-1

MURPHY, J. (ESS-3)

45-1

MURPHY, JAMES T. (CMB-10) $14-43$

MURRAY, JOHN G. 26-20

MURRELL, M.T. 48-69

MUTSCHLECNER, J. PAUL (X-5) 48-5

MYDOSH, JOHN A. 38-29

NAGASE, F. 48-62

NAGLE, DARRAGH E. (MP-DO) 49-18 49-19

50-82

$51-33$

$51-34$

NALESSO, GIANFRANCO

17-16

NANDA, SIRISH 50-15

NARANJO, CLEO M. (LS-3) 2.54

NARANJO, RAMONCITA (E-11)

$11-3$ 


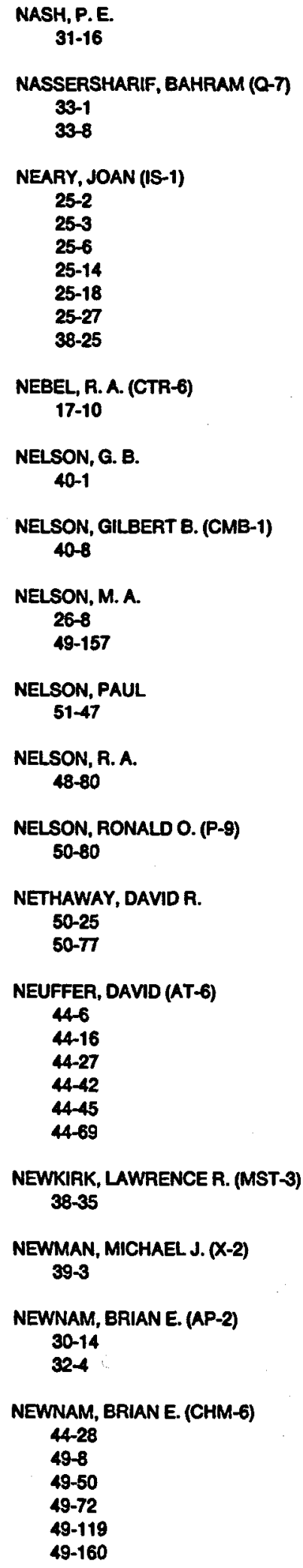

NASH,P.E. 31-16 NASSERSHARIF, BAHRAM $(Q-7)$
$33-1$

33-8

NEARY, JOAN (IS-1)

25-2

25-3

25-6

25-14

25-18

25-27

38-25

NEBEL, R. A. (CTR-6)

17-10

NELSON, G. B.

40-1

NELSON, GILBERT B. (CMB-1) 40-8

NELSON, M. A.

26-8

49-157

NELSON, PAUL

51-47

NELSON, R. A.

48-80

NELSON, RONALD O. (P-9) $50-80$

NETHAWAY, DAVID R.

50-25

$50-77$

NEUFFER, DAVID (AT-6)

44-6

44-16

44-27

44-42

44-45

44-69

NEWKIRK, LAWRENCE R. (MST-3) 38-35

NEWMAN, MICHAEL J. (X-2) 39-3

NEWNAM, BRIAN E. (AP-2)

30-14

$32-4$

NEWNAM, BRIAN E. (CHM-6)

44-28

49-8

49-50

49-72

49-119

49-160

NEWTON, THOMAS W. (INC-2) 5-16

NG, $R$.

50-94

NGAI,K.L.

49-125

NICHOLS, BASIL (T- $\eta$

39-42

NICHOLSON, DWIGHT R. 48-104

NICHOLSON, NICHOLAS (Q-2) 53-9

NICHOLSON, ROBERT W. 21-3

21-4

NICKEL, GEORGE H. (X-2) 47-2

NICKOLS, JOYCE W. (LS-3) 2-46

NICOLAENKO, BASIL (T-7/CNLS) 39-43

39-94

NICOLAS, K. R. 48-52

NIEMCZYK, THOMASM.

NIETO, MICHAEL MARTIN (T-8)

47-22

$51-7$

51-9

51-14

51-25

$51-28$

51.30

NIEUWENHUYS, G. J. 38-29

NILSSON, L.R.

$50-55$

NIX, JAMES R. (T-9)

$50-8$

$50-30$

50-50

$50-88$

50-106

50-122

NIXON, KERMIT V. (Q-2)

53-51

53-53
NOCHUMSON, DAVID H. (S-4)

16-2

16-3

16-31

16-34

16-37

NOEL, BRUCE W. (WX-10)

26-8

31-20

31-26

$31-42$

NOGAR, NICHOLAS S. (CHM-2)

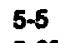

$5-63$

47-30

47-49

47-50

49-56

NORMAN, M. L. $(x-8)$ 39-3

NORRIS, O. ROY (AT-T)

49-50

NORRIS, THOMAS L (INC-7) 50-53

NORTON, J.

$50-69$

NORTON, KENNETH A.

47-63

NUNNALLY, WLLLAM C. (E-11)

14-1

$14-38$

$14-41$

14-52

$14-68$

$31-9$

NUNZ, GREGORY J. (ESS-DOT) 22-4

NUSSINOV, RUTH (T-10) 2-45

NUSSINOV, S. (T-8)

50-44

51-20

NUTTALL, HERBERT E. 42-14

NUTTALL, HERBERT E. 42-15

NYHAN, JOHN W. (LS-6)

16-10

42-3

$42-6$

42-24

$42-30$ 
NYHOLM, RALF

48-75

O'BRIEN, HAROLD A. (INC-3) 5-43

O'DELL, R. DOUGLAS (X-6)

18-12

24-14

39-118

39-119

O'ROURKE, PETER J. (T-3)

39-22

$39-81$

OAKES, WILLIAM R. (WX-11) 39-91

OBENSHAIN, F. E. $50-45$ $50-94$

OGARD, ALLEN E. (INC-7) 42-9

OGLE, J. W. (P-14)

14-9

31-30

$31-44$

OH, SE-JUNG

47-12

55-1

OHKUBO, YOSHI (CNC-11) 50-65

OHM, HENNER (P-3) $50-45$

50-84

50-105

OHNUMA, H. 50-21

OKA, TAKAMITSU (T-5) 51-42

OKINAKA, RICHARD T. (LS-3) 2-1 2-34

$2-46$

OLDENBORG, RICHARD C. (CHM-4) 5-41

OLA, $H$. 49-154

OLINGER, BARTON W. (M-6) 5-69

$38-50$

49-14

49-43
OLSEN, CLAYTON E. (MST-13)

31-38

$49-67$

$50-80$

OLSEN, KENNETH H. (ESS-3)

16-7

$16-8$

$16-9$

OLSEN, OLE H.

39-12

ONSTOTT, EDWARD I. (CHM-2)

OONA, HENN (P-7) 30-19

ОOTHOUDT, M. A. (MP-1) 39-65

50-69

ORTH, CHARLES J. (INC-11)

16-35

$16-42$

50-113

ORTIZ, JOHN P. (HSE-5)

28-18

OSBORNE, W. Z. 49-38

OSTENAK, CARL ANDREW (Q-4) 63-11

53-14

OTSUKA, TAKAHARU (T-5) 49-1

OTT, HANS R.

38-18

38-23

$38-46$

OVEREND, JOHN 47-23

OVERTON, WILLIAM C., JR. (P-10)

14-4

$14-20$

14-40

49-4

49-46

49-127

OVRUT, BURT A. 49-150

PACK, RUSSELLT. (T-12)

47-18

$51-6$

PAFFETT, MARK THOMAS (E-11) 5-61
PAINE, ROBERT T., JR.

5-39

5-77

PALMER, A. R.

16-35

PALMER, BYRON A. (CHM-1)

5-75

5-86

47-32

PAN, PAUL Y. (Q-6)

$$
\text { 50-71 }
$$

PARKER, JACKL (Q-1)

53-4

53-58

PARKER, JERALDV. (P-7)

14-12

14-42

PARKER, JOSEPH R. (P-5)

31-7

31-19

PARKINSON, WILLIAM J. (WX-4) 39-106

43-2

43-3

PARKS, RONALD D. 47-71

PARSONS, W. M. (CTR-9) $14-32$

PASCHMANN, GOTZ

48-39

48-111

PASSELL, LAURENCE 47-39

PATERNOSTER, RICHARD R. $(X-7)$ 39-61

PATTERSON, CHRIS W. (T-12)

$$
\text { 47-13 }
$$

47-53

PATTERSON, JAMES H. (CHM-1) 40-1

40-8

PATTON, ROBERT D. (AT-5) 44-20

PAULETTA, G. 50-59

PAULS, R. E. 5-60 
PAULTER, NICHOLAS G. (E-11)

$14-30$

14-31

$31-21$

31-29

38-13

PAYNE, GERALD L.

48-104

50-28

50-91

PEARSON, CHRISTOPHER F. (ESS-3)

21-7

22-5

45-1

PEAsLeE, A. T., JR. (S-4)

11-1

PEDERSON, RAYMOND A. (Q-2) 9-9

9-15

. $31-10$

PELIZZONE, $M$.

38-6

PENDERGRASS, JOHN H. (S-4)

30-1

$30-2$

30-12

30-20

PENDLEY, ROBERT E. (ADPA) 25-7

PENNEMAN, ROBERT A. (INC-4) 5-1

PERATT, ANTHONY L. $(X-8)$ 39-21

49-120

PEREA, JACOB A. (WX-10) 31-14

PERELSON, ALAN S. (T-10) 2-21

2-32

$2-33$

2-37

2-40

PERKINS, BETTY A. (LS-6) 42-6

PERRAssini, M.

31-30

PERRIN, DANIEL R. (HSE-8) 5-53

5-54

PERRIN, RICHARDE. (INC-7)

5-28

16-11
PERROUD, J. P.

50-67

PERSON, WILLIS B.

47-65

PESME, DENIS

49-144

PETER, WILLIAM

49-65

PETER, WILLAM K. (X-8)

44-68

49-57

49-135

PETERSON, DEAN E. (MST-5) 38-43

PETERSON, DENNIS R. $(W X-4)$ 14-12

PETERSON, EUGENE J. (CHM-1) 5-15

PETERSON, R. J. 50-12

PETERSON, R.T. 14-9 31-15

PETROVIC, JOHN J. (MST-5) 38-14

38-27

38-28

38-35

PETSCHEK, ALBERT G. (P-DO)

48-48

48-49

$50-82$

PETTITT, ROLAND A. (ESS-4)

21-3

21-4

21-10

PHILLIPS, JAMES A. (CTR-DO)

17-8

17-10

PHILUIPS, JOHN R. (Q-1)

53-27

53-54

PHILUPS, THOMAS TERRENCE $(w X-4)$

43-1

43-2

43-3

PHILLPOT, SIMON R.

38-33

49-5

PIANETTA, $P$.

31-35
PIANETTA, PIERO
$14-26$
PIASETZKY, ELI
$50-16$
$50-24$
$50-66$
$50-93$
$50-101$
$50-107$

PICARD, RICHARD R. (S-1)

39-25

53-25

53-26

PICKLESIMER, $A$.

50-11

PILAT, JOSEPH F. (ADPA-SA) 25-7

PILLAY, K. K. S. (Q-4)

53-25

53-26

PILLMORE, C. L. 16-42

PIMBLEY, GEORGE H. (T-14) 3-17

39-100

PINES, DAVID 49-149

PITCHER, ERIC J. 48-102

PLANNER, H. N. (ESS-2) 48-86

PLASIL, F. 50-45

Plassmann, Eugene A. (Q-2) $9-4$

$9-9$

9-15

31-10

PLATTS, D. A. (CTR-5)

18-10

18-17

PLENDL, H.S.

50-69

POLETTO, G. 48-43

POLZER, WILFRED L. (LS-6) 42-10

POMRANING, GERALD C. $(x-7)$ 39-62 


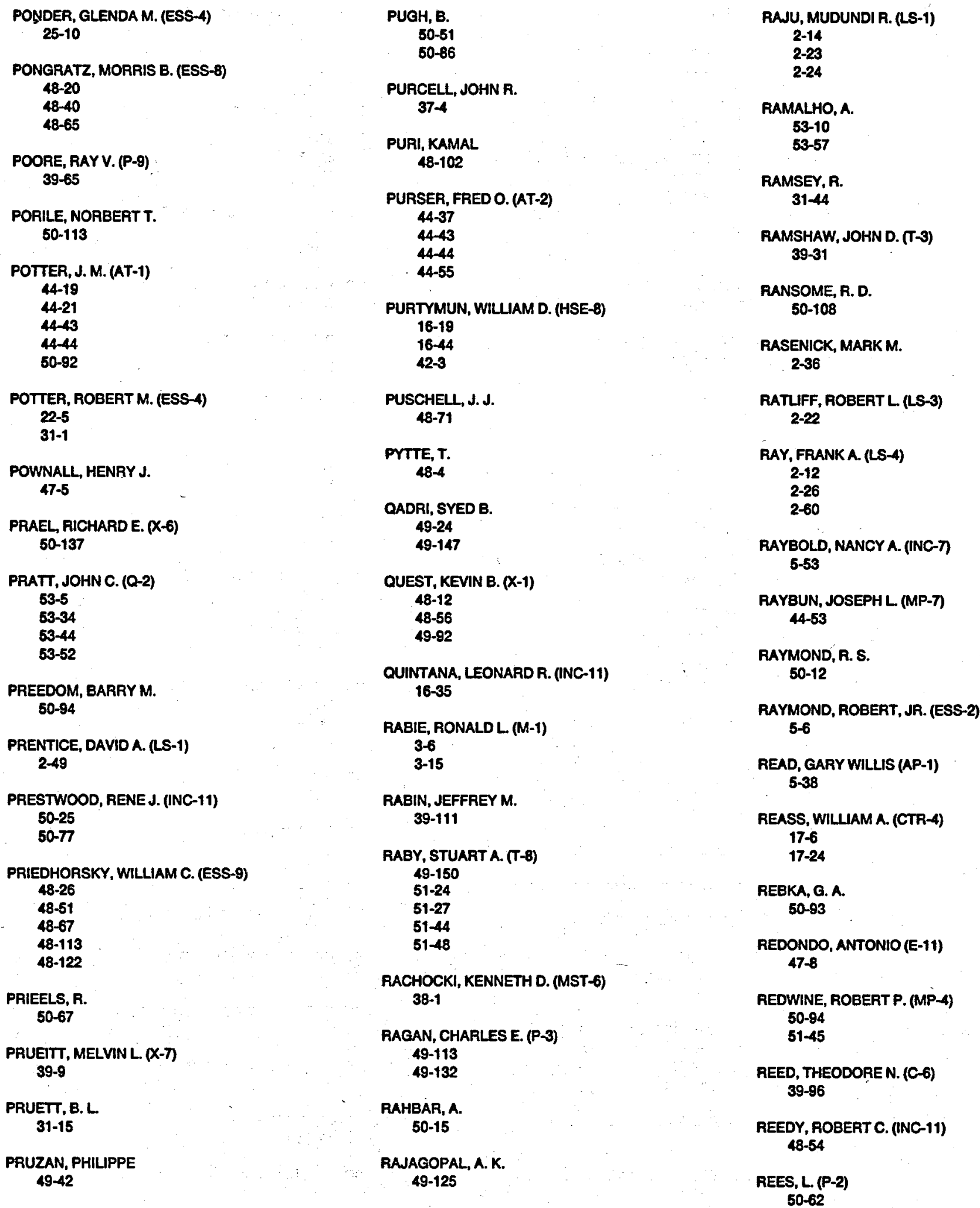

PRIEELS, $R$. 50-67

PRUEITT, MELVIN L. $(X-7)$ 39-9

PRUETT, B. L 31-15

PRUZAN, PHILIPPE 49-42

PUCH, B.

$50-51$

$50-86$

PURCELL, JOHN R.

$37-4$

PURI, KAMAL 48-102

PURSER, FRED O. (AT-2)

44-37

44-43

44-44

44-65

PURTYMUN, WILLIAM D. (HSE-8)

16-19

16-44

42-3

PUSCHELL, J.J. 48-71

PYTTE, T.

48-4

QADRI, SYED B.

49-24

49-147

QUEST, KEVIN B. (X-1)

48-12

48-56

49-92

QUINTANA, LEONARD R. (INC-11) 16-35

RABIE, RONALD L. (M-1)

3-6

3-15

RABIN, JEFFREYM.

39-111

RABY, STUART A. (T-8)

49-150

$51-24$

51-27

51-44

$51-48$

RACHOCKI, KENNETH D. (MST-6) 38-1

RAGAN, CHARLES E. (P-3) 49-113

49-132

RAHBAR, A. 50-15

RAJAGOPAL, A. K. 49-125

RANU, MUDUNDI R. (LS-1)

2-14

$2-23$

2-24

FAMALHO, A.

53-10

53-57

RAMSEY, $R$

$31-4$

RAMSHAW, JOHN D. (T-3) 39-31

RANSOME, R. D. 50-108

RASENICK, MARKM. 2.36

RATUFF, ROBERT L. (LS-3) 2-22

RAY, FRANKA. (LS-4) 2-12

2-26

$2-60$

RAYBOLD, NANCY A. (INC-T) 5-53

RAYBUN, JOSEPH L (MP-7) 44-53

RAYMOND, R.S. 50-12

RAYMOND, ROBERT, JR. (ESS-2) 5-6

READ, GARY WILLIS (AP-1) 5-38

REASS, WILLIAM A. (CTR-A) 17-6

17-24

REBKA, G. A.

$50-93$

REDONDO, ANTONIO (E-11) 47-8

REDWINE, ROBERT P. (MP-4) 50-94

$51-45$

REED, THEODORE N. (C-6) 39-96

REEDY, ROBERT C. (NC-11) 48-54

REES, L. (P-2)

50-62 
REEVES, GARY ALAN (MST-6) $30-3$

REGAZZONI, GILLES (MST-5) $38-47$

REGISTER, D.F. 47-20

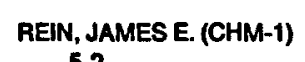

REXROTH, VERNER G. (MST-7) 18-16

REYES, MICHELE (H-14) 28-25

REYES, MICHELE (HSE-14) 28-3

RHORER, RICHARD L (MEC-4) 14-65 RICH, JEANETTE A. (IS-4)
26-12

RICH, MARVIN (X-5) 49-113

RICHAROS, PETER M. 47-62

RICHTER, JOHN L. (NSP-WP) 49-142

RICKETTS, C. I. 24-7

RIDER, B. F. 50-123

RILEY, P. J. 50-21 50-108
RINARD, PHILLIP M. (Q-1)

33-10

53-27

53-54

RINEHART, GARY H. (CMB-3)

38-48

RINKER, GEORGE A., JR. (T-4) 49-33

49-151

RIS, FRED.

$39-46$

RISLOVE, SETH E. (MP-8) 44-26

RISTINEN, R. A. 50-12

RITCHIE, B. G. 50-94

RITCHIE, JAMES P. (T-14) 47-44

RIVERA, RAYNALDO V. (E-5) 25-23

RIVERA, THOMAS (M-1) 3-6

ROBERSON, N. R. 50-55

ROBERTSON. ROBERT Q. H. (P-3) 50-135

ROBINSON, BRUCEA.

21-9

$22-8$

ROBINSON, EDWARD E. (P-3) 49-113

ROBINSON, RICHARD D. (H-8) 5-53 5-54

ROBINSON, ROBERT A. (P-8) 50-73

ROBSON, A.E. 48-57

ROCKWOOD, STEPHEN D. (NSP/IF) 30-8 49-22 50-121

RODEBAUGH, R.F. 50-21

RODRIGUEZ, LA VERNE L (IS-4) 26-12
ROFER-DE POORTER, CHERYL K. (ESS-2) 5-84

ROGERS, JOHN D., JR. (CTR-9)

13-1

$37-4$

37-7

37-12

$37-13$

37-15

ROGERS, PAMELA S. Z. (INC-7)

16-14

48-69

ROGERS, RAYMOND N. (M-1) 50-136

ROHR, DANA L. (MST-5)

$37-10$

38-8

$38-43$

$38-44$

ROKOP, DONALD J. (INC-7)

50-25

50-53

ROLFE, J. R.

49-18

ROMANOWSKI, T.A. 50-92

RON, AMIRAM

49-65

ROOF, RAYMOND BRADLEY, JR. (MST-5) 5-69

38-14

$38-44$

ROOS, LAURIES.

2.37

ROSEN, S. P. (T-8)

$51-4$

$51-11$

51-12

$51-22$

$51-50$

ROSEN, SIMON PETER (T-DO) 51-19

ROSENBLATT, GERD M. (CHM-DO) 5-3

ROSENBLUM, STEPHEN S. 49-87

ROSMUS, PAVEL 47-36

ROSS, D. C. (E-8) 39-6 
ROSTOKER, NORMAN

49-65

ROTA, GIAN-CARLO 39-28

ROTH, E. JIL

$$
\text { 2-54 }
$$

ROUSSEL-DUPRE, ROBERT (ESS-7) 48-52 48-65

ROWLAND, MARY M.

$$
\text { 16-27 }
$$

$16-43$

ROWLEY, JOHN C. (ESS-4) 21-3

21-4

21-10

RUDIGIER, $H$. 38-46

RUDY, R. J. 48-71

RUMINER, KIT (MP-DO) 49-116

RUNDBERG, ROBERT S. (INC-11) 42-14

42-15 50-37

RUNDBERG, VIRGINIAL L (INC-11) 5-16

RUSBRIDGE, MICHAEL G.

17-1

$17-23$

RUSSELL, C.T.

48-39

48-72

48-73

48-111

RUSSELL, GARY J. (P-9) $31-8$

RUSSO, P.A. (Q-1) 53-46

RUSTHOI, DANIEL $P$. 44-37

RUSTHOI, DANIEL'P. (AT-2) 39-112

RUTH, T. J. 5-22
RYAN, ROBERT R. (INC-4)

5-58

5-59

5-76

5-77

47-7

RYAN, WILLIAM

48-79

SAHA, A. 50-19

SALAZAR, JOHN (H-8) 16-32

SALAZAR, KENNETH V. (INC-4) 5-77

SALZMAN, GARY C. (LS-DO) 2-31 2-52

SAMSEL, RICHARD W. $2-33$

SAMUELSEN, MOGENS R. 39-12

SAMWER, KONFAD 50-64

SANĆHEZ, J. A. (M-1) 3-12

SANDBERG, V.D. (MP-4)

49-18

$50-94$

51-14

$51-34$

SANDER, OSCAR R. (AT-2) 44-37

SANDER, ROBERT K. (CHM-4)

47-35

49-22

SANDERS, GARY H (MP-13)

49-18

49-62

$51-8$

$51-34$

SANDERS, WILLIAM MORT (HSE-7) 42-13

SANDERSON, JAMES G., JR. (X-7) 39-106

SANDFORD, ELSIEL. (Q-1)

$$
\text { 53-46 }
$$

SANDFORD, MAXWELL T. (ESS-5)

$16-6$

16-18

48-93
SANDOVAL, ABAD ELI (IS-DO) 26-10

SANDOVAL, RICHARD R. (S-6) 26-7

SAPONARA, NIJOLE M. (CHM-1) 6-12

SARANGI, SUNIL (P-10)

14-20

49-46

SARJEANT, WALTER J. (E-11) $14-36$

SARJEANT, WALTER J. (E-4)

31-22

$31-23$

SARRACINO, ROBERT 48-48

SATUA, SUSHIL K. $47-60$

SAUNDERS, GEORGE C. (LS-4)

$2-44$
$2-60$

SAYLOR, WILLIAM W. (S-4)

30-1

30-2

SCHAAD, P.A.

$37-4$

SCHALLER, STUART C. (MP-1) 44-40

SCHERMER, ROBERT I. (CTR-9) $37-4$

37-13

37-14

37-15

-

SCHEURER, BRUNO (CNLS) 39-94

SCHEUTER, FRANZ J. 50-122

SCHIFERL, DAVID (M-6) 5-46

$47-42$

49-137

SCHIFERL, SHEILA K. $(X-4)$ 47-59

SCHILLACI, MARIO E. (MP-3)

38-11

38-29

47-37

SCHILLING, J. 2-13 


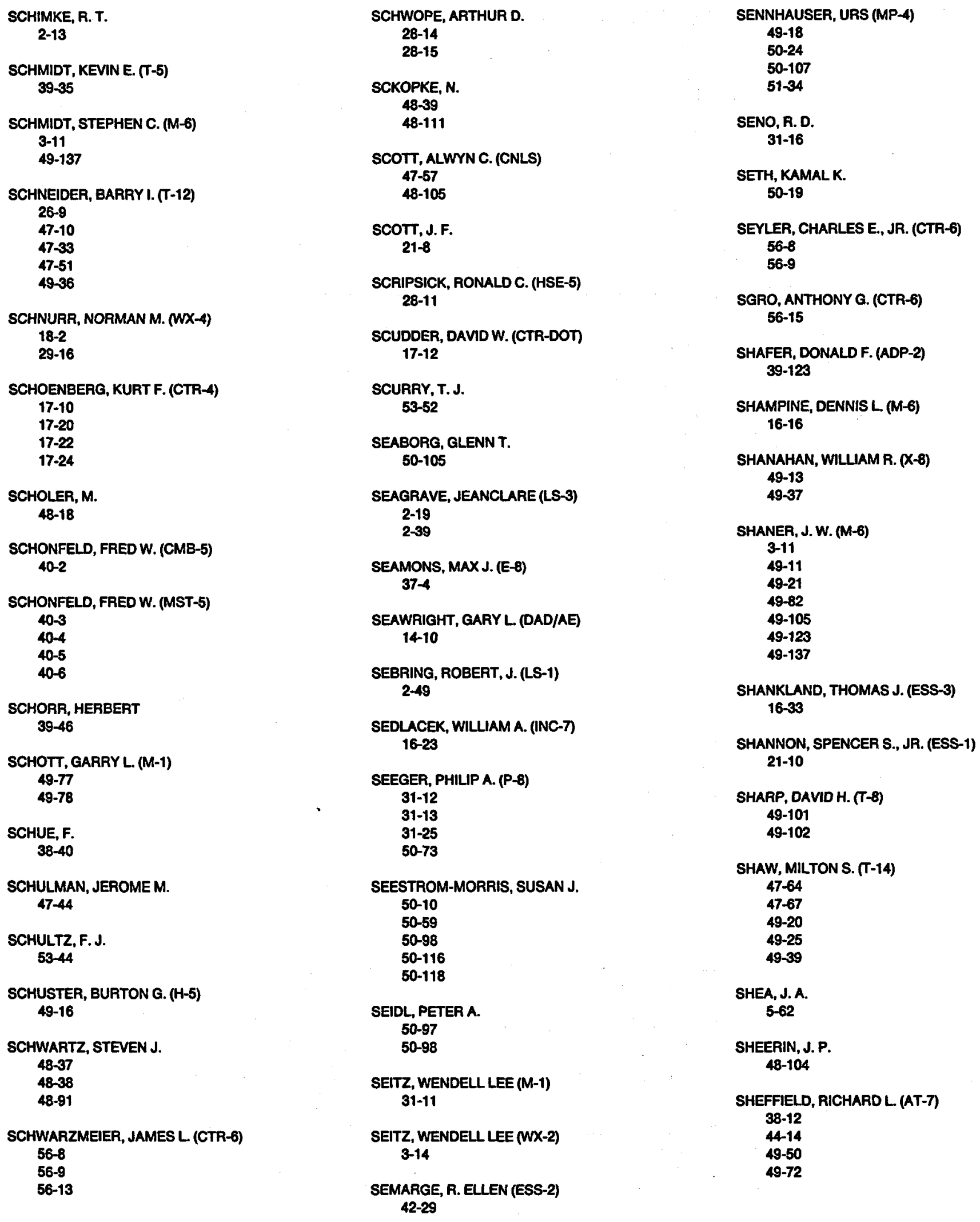


SHEINBERG, HASKELL (MST-6)

- $38-34$

SHELTON, R. N. (AP-4)

31-15

SHEPPARD, MAURICE G. (T-12) 47-54

SHERA, E. BROOKS (P-3)

39-65

50-34

50-51

$50-74$

50-75

50-86

50-102

50-131

SHERIDAN, CONNIE J. (IS-4) 26-12

SHERMAN, J. D.

$$
\text { 44-36 }
$$

SHERMAN, JOSEPH D. (AT-2) 44-58

SHERMAN, ROBERT H. (MST -3) 18-6

SHERWOOD, ARTHUR R. (CTR-5)

18-9

18-10

18-13

18-17

SHERWOOD, EUGENE G. (CTR-11) 17-14

$17-36$

SHIAH, SHOOU-SHINN (P-10) 49-98

SHIH, CHIA CHANG

$51-49$

SHIPLEY, JAMES P., JR. (Q-DO/SG)

39-7

39-56

39-101

53-38

SHISSLER, D.

53-42

SHLACHTER, JACK S. (CTR-DOT) 17-11 17-12

SHUL'GIN, B. V. 50-130

SHULMAN, S.

48-123

48-124

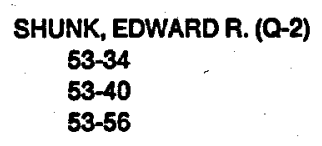

SHYAMSUNDER, E.

SIBECK, D. G.

48-25

SICILIANO, EDWARD R.

50-4

50-22

$.50-89$

SIEGEL, HOWARD J.

$39-46$

SIEGEL, PETER $B$.

$50-68$

SIEMON, RICHARD E. (CTR-3)

17-14

17-21

$17-36$

SIERK, ARNOLD J. (T-9)

50-30

50-84

50-122

SILBAR, RICHARD R. (T-5)

50-66

50-81

50-117

SILVER, RICHARD N. (P-8)

49-117.

49-118

SIMANTON, J.R.

16-10

$16-30$

SIMMONS, JAMES E. (P-T)

50-43

SIMMONS, LEONARD M., JR. (T-DO)

51-32

$51-41$

SIMMONS, MARGARET L. (C-10) 39-67

SIMONS, DAVID J. (ESS-7)

48-40

48-80

SIMS, JAMES R. (MP-8)

44-26

SINCLAIR, A. RICHARD

21.3

21-4

SINGER, SIDNEY (P-DO)

30-10

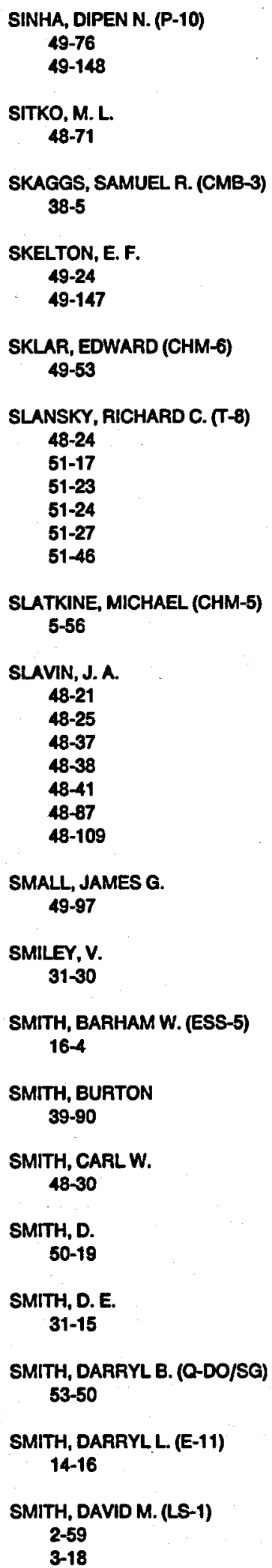




\begin{tabular}{|c|c|}
\hline $\begin{array}{l}\text { SMITH, DAVID R. (HSE-6) } \\
9-11 \\
9-17\end{array}$ & $\begin{array}{l}\text { SNELL, CHARLES M. }(X-8) \\
39-88 \\
39-117\end{array}$ \\
\hline $\begin{array}{c}\text { SMITH, E. J. } \\
48-21 \\
48-25 \\
48-32 \\
48-37 \\
48-38 \\
48-72 \\
48-73 \\
48-87 \\
48-88 \\
48-109\end{array}$ & $\begin{array}{l}\text { SNYDER, CONNIE (IS-1) } \\
25-1 \\
25-8 \\
25-9 \\
25-16 \\
25-20 \\
25-24 \\
26-1 \\
\text { SNYDER, LAWRENCE } \\
\text { 39-46 }\end{array}$ \\
\hline $\begin{array}{c}\text { SMITH, F. W. } \\
24-5\end{array}$ & $\begin{array}{l}\text { SNYDER, WILLIAM H. } \\
16-41\end{array}$ \\
\hline $\begin{array}{l}\text { SMITH, HARRY L. } \\
\quad 49-64\end{array}$ & $\begin{array}{l}\text { SODERHOLM, SIDNEY C. (HSE-5) } \\
28-11\end{array}$ \\
\hline $\begin{array}{l}\text { SMITH, HASTINGS A., JR. (Q-1) } \\
53-35\end{array}$ & $\begin{array}{l}\text { SOLEM, JOHNDALE C. (T-DO) } \\
\begin{array}{l}2-62 \\
39-69\end{array}\end{array}$ \\
\hline $\begin{array}{l}\text { SMITH, HORACE V. (AT-2) } \\
\text { 44-58 }\end{array}$ & $\begin{array}{c}\text { 49-159 } \\
\text { SOLLD, JONE. (P-5) }\end{array}$ \\
\hline $\begin{array}{l}\text { SMITH, JAMES L. (MST-5) } \\
\text { 38-6 }\end{array}$ & 49-72 \\
\hline $\begin{array}{l}38-11 \\
38-18 \\
38-32 \\
38-37 \\
38-41 \\
38-46 \\
38-49\end{array}$ & $\begin{array}{l}\text { SOMMER, WALTER F., JR. (MP-13) } \\
37-11 \\
38-3 \\
38-45 \\
44-7 \\
44-60\end{array}$ \\
\hline $\begin{array}{l}47-71 \\
49-103 \\
49-156\end{array}$ & $\begin{array}{l}\text { SOPER, ALAN K. (P-8) } \\
\text { 31-38 }\end{array}$ \\
\hline$\underset{50-99}{\text { SMITH, L. E. }}$ & $\begin{array}{l}\text { SORAN, PATRICK D. }(X-6) \\
9-7\end{array}$ \\
\hline $\begin{array}{l}\text { SMITH, NORMAN L. } \\
\text { 50-25 }\end{array}$ & $\begin{array}{l}\text { SOURKES, A.M. } \\
50-138\end{array}$ \\
\hline $50-77$ & $\begin{array}{l}\text { SPALL, W. DALE (LS-1) } \\
2-49\end{array}$ \\
\hline $\begin{array}{l}\text { SMITH, PHILLUP R. } \\
24-7\end{array}$ & $\begin{array}{l}\text { SPARKS, WARREN M. (X-5) } \\
\text { 48-114 }\end{array}$ \\
\hline $\begin{array}{c}\text { SMITH, RUSS C. (Q-7) } \\
31-44 \\
33-12\end{array}$ & $\underset{51-45}{\text { SPECHT, J.R. }}$ \\
\hline $\begin{array}{l}\text { SMITH, STEVEN A. (C-6) } \\
2-10 \\
2-43\end{array}$ & $\begin{array}{l}\text { SPEIR, LESLIE GAYLE (Q-1) } \\
14-5\end{array}$ \\
\hline $\begin{array}{l}\text { SMITH, SUZANNE T. (Q-8) } \\
\text { 53-7 }\end{array}$ & $\begin{array}{l}\text { SPENCER, ROSS L. (CTR-6) } \\
56-12\end{array}$ \\
\hline$\underset{49-130}{\text { SMITH, W. W. }}$ & $\begin{array}{c}\text { SPILLER, EBERHARD } \\
48-121 \\
49-107 \\
49-108\end{array}$ \\
\hline $\begin{array}{l}\text { SMYTH, JOSEPH R. (ESS-1) } \\
\text { 16-26 }\end{array}$ & $\begin{array}{c}\text { SPINKA, H. } \\
49-81\end{array}$ \\
\hline
\end{tabular}

SPOUGE, JOHN L. $(T-10)$
$5-4$
$39-66$

SPRINGER, EDWARD A. (OS-4) 39-27

SPRINGER, ROBERT W. (MST-6) 38-1

SPRINGER, THOMAS E. (E-11)

$11-3$

38-13

SPRINKLE, JAMES K., JR. (Q-1)

53-1

53-20

SRINIVASAN, S. (E-11)

5-17

11-2

11-3

SRIVASTAVA, S. K.

47-14

STACY, HOWARD L. (M-1) $31-11$

STAFFORD, CAROLYN G. (LS-DO) 2-2

STALLINGS, E. A. (LS-6) 16-39

STALLINGS, R. L. $2-4$

STALNAKER, NELSON D. (CHM-1) 40-1

STAMPFER, JOSEPH F., JR. (HSE-5) 28-4

STANEK, $R$.

49-81

STANLEY, JAMES T. (MST-5)

38-10

38-20

38-21

STARNER, JOHN W. (INC-5) 50-54

STARRFIELD, $S$. 48-114

STAUDHAMMER, KARL P. (MST-5) 30-3

38-2

$38-8$

$38-50$

STEENMAN-CLARK, LOIS 48-2 


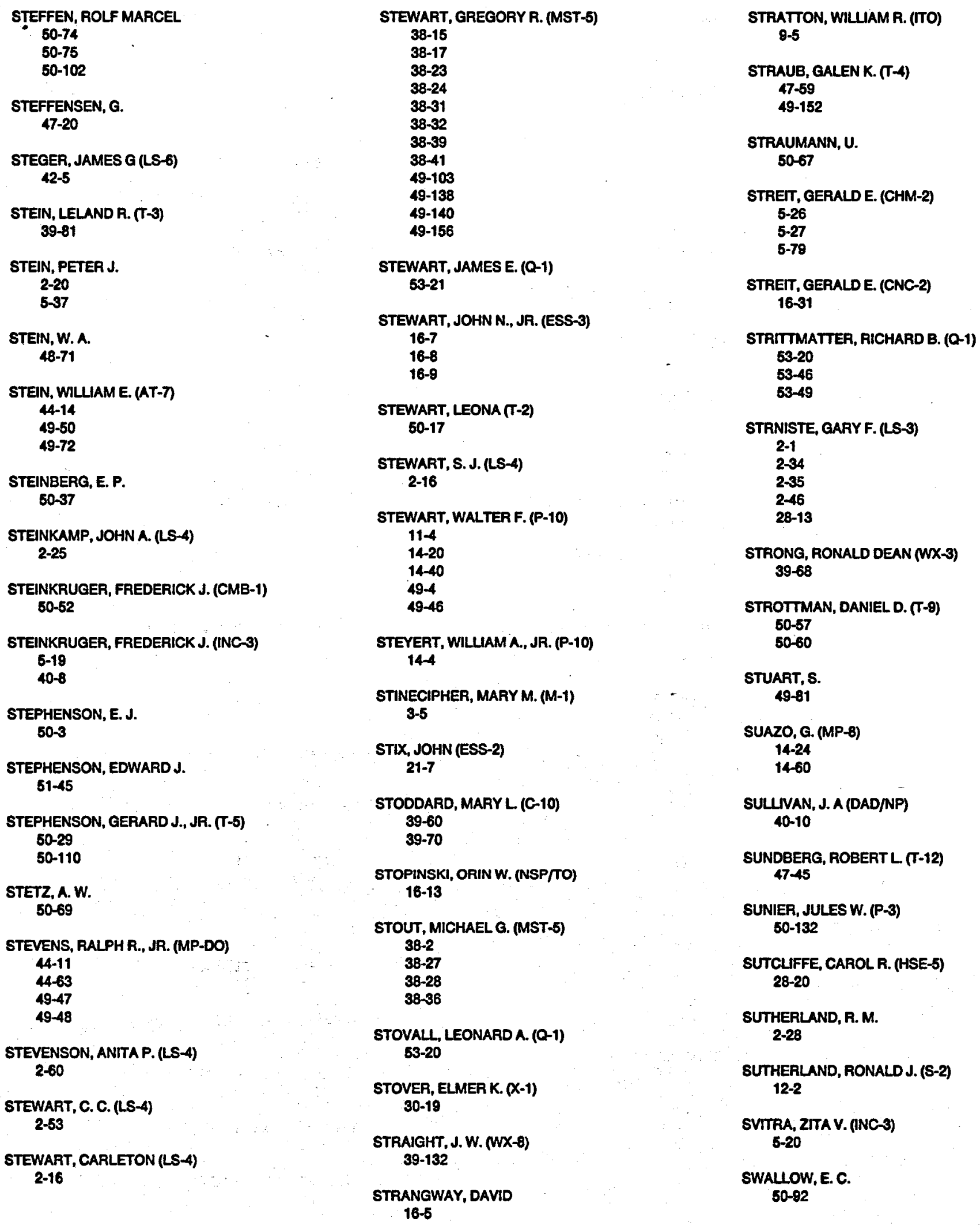




\begin{tabular}{|c|c|}
\hline $\begin{array}{l}\text { SWANN, THOMAS A. (P-5) } \\
\text { 49-72 }\end{array}$ & $\begin{array}{l}\text { TANG, P. K. }(X-7) \\
24-7 \\
39-116\end{array}$ \\
\hline \multicolumn{2}{|l|}{ SWANSEN, JAMES E. (0-1) } \\
\hline $14-61$ & TAPE, JAMES W. $(Q-1)$ \\
\hline 53-13 & $53-49$ \\
\hline \multicolumn{2}{|l|}{$53-57$} \\
\hline \multicolumn{2}{|l|}{ SWANSON, BASILI. (INC-4) } \\
\hline \multicolumn{2}{|l|}{ 5-29 } \\
\hline $5-47$ & TAYLOR, ANDREW D. (P-8) \\
\hline $5-48$ & $31-38$ \\
\hline 5-55 & $50-72$ \\
\hline $47-7$ & $50-73$ \\
\hline \multicolumn{2}{|l|}{$47-31$} \\
\hline $47-40$ & TAYLOR, THOMAS N. (CHM-2) \\
\hline $47-63$ & $5-73$ \\
\hline $\begin{array}{l}\text { SWENSON, L.W. } \\
\text { 50-69 }\end{array}$ & $\begin{array}{l}\text { TAYLOR, WAYNE A. (INC-3) } \\
\text { 5-43 }\end{array}$ \\
\hline SWIFT, GREG W. (P-10) & TAYLOR, WILLIAM B. \\
\hline \multicolumn{2}{|l|}{$49-80$} \\
\hline \multirow{3}{*}{$\begin{array}{l}\text { SYDORIAK, STEPHEN G. (P-10) } \\
49-104\end{array}$} & TAYLOR, WILLAM J., JR. \\
\hline & $25-26$ \\
\hline & TEASDALE, WULAM A. (P-3) \\
\hline \multirow[t]{2}{*}{$\begin{array}{l}\text { SYMON, KEITH R. (CTR-6) } \\
56-5\end{array}$} & $49-113$ \\
\hline & TELLE, JOHN M. (CHM-6) \\
\hline $\begin{array}{l}\text { SZE, ROBERT C. (CHM-5) } \\
49-63\end{array}$ & $\begin{array}{l}5-18 \\
49-31\end{array}$ \\
\hline \multirow{2}{*}{$\begin{array}{l}\text { TABATABAEI, S. D. } \\
\text { 30-15 }\end{array}$} & $\begin{array}{l}\text { TERRELL, NELSON JAMES (ESS-9) } \\
\text { 48-26 }\end{array}$ \\
\hline & $\begin{array}{l}48-26 \\
48-51\end{array}$ \\
\hline \multirow{2}{*}{$\begin{array}{c}\text { TAKAGI, AKIRA } \\
44-39\end{array}$} & $48-67$ \\
\hline & $\begin{array}{r}48-113 \\
48-119\end{array}$ \\
\hline $\begin{array}{c}\text { TALAGA, R. L. } \\
50-92\end{array}$ & $48-122$ \\
\hline $51-34$ & $\begin{array}{l}\text { TESTER, JEFFERSON W. } \\
21-9\end{array}$ \\
\hline \multirow[t]{2}{*}{$\begin{array}{l}\text { TALBERT, W. L., JR. (NC-11) } \\
\text { 50-54 }\end{array}$} & $22-8$ \\
\hline & TESTER, JEFFERSON W. (ESS-4) \\
\hline \multirow[t]{2}{*}{$\begin{array}{l}\text { TALLEY, BEVERLY (MP-DO) } \\
49-116\end{array}$} & $27-1$ \\
\hline & THALER, R. M. \\
\hline \multirow{2}{*}{$\begin{array}{l}\text { TAN, TAI HO (P-4) } \\
\text { 30-7 } \\
48-65\end{array}$} & $50-11$ \\
\hline & $\begin{array}{l}\text { THAYER, GARY R. (S-4) } \\
\text { 18-18 }\end{array}$ \\
\hline \multirow{3}{*}{$\begin{array}{l}\text { TANAKA, NOBUYUKI (MP-10) } \\
50-62 \\
50-116\end{array}$} & 24-11 \\
\hline & $30-1$ \\
\hline & $52-2$ \\
\hline \multirow{3}{*}{$\begin{array}{l}\text { TANAKA, YASUTOSHI (P-7) } \\
50-74 \\
50-75 \\
50-102\end{array}$} & THAYER, NINA N. \\
\hline & $31-20$ \\
\hline & $\begin{array}{r}\text { THEIS, S. } \\
48-54\end{array}$ \\
\hline $\begin{array}{l}\text { TANDY, P.C. } \\
\text { 50-11 }\end{array}$ & \\
\hline
\end{tabular}

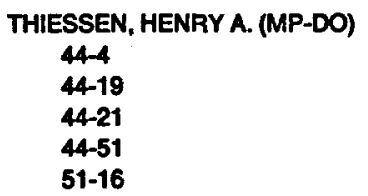

THOMAS, KIMBERLY W (INC-11) 50-53 50-77

THOMAS, ROBERT G. (H-DO) 28-23

THOMAS, SCOTT J. (AP-5) 32-2

THOMAS, W.S. 33-13

THOMPSON, JOE D. (P-10) 38-19 38-37 $38-49$ $49-74$

THOMPSON, JOE L (INC-11) 42-11

THOMPSON, KENNETH A. (CHM-2) 5-73

THOMPSON, LOIS J. (PA-7) 25-21

THOMPSON, MELVIN C. (E-8) $14-63$

THOMSEN, MICHELLE F. (ESS-8) 48-16

48-56 
THORN, ROBERT N. (DIR-O)

$$
\text { 26-16 }
$$

39-51

THORNE, JAMES $M$.

$$
\text { 32-3 }
$$

TIEE, JOE J. (CHM-4) 47-35

TIERNEY, GAIL D. (HSE-8) $16-38$

TIERNEY, GAIL D. (LS-6)

42-22

42-23

TIETJEN, GARY L. (S-1) 28-3

TILLERY, MARVIN I. (HSE-5) 28-11

TIMMONS, G. 53-42

TNGG, C. S. 49-67

TIPPENS, W. B. 50-108

TISINGER, E. W. (Q-2) 9-8 9-10 63-22

TJON, JOHN (T-5) 50-78

TOBEY, ROBERT A. (LS-1) 2-11

TOBEY, ROBERT A. (LS-4) 2-5

TOÉVS, J. W. (P-14)

$$
\text { 31-16 }
$$

TOKAR, ROBERT L. (ESS-8) 48-34

48-91

TOKI, H. 50-22

TOKITA, NOBUHIKO (LS-1) 2-24 28-13

TOMUSIAK, EDWARD L. 50-114

TONKS, DAVIS L. (T-11) 47-61

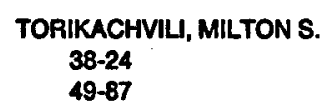

TRAN, M.T. 50-67

TRAVIS, BRYAN J. (ESS-5) $39-58$ 39-84 39-113

42-14

42-15

TRAVIS, GAYLE LEE (LS-4)

$$
\text { 2-15 }
$$

2-60

TRAVIS, JOHN R. (T-3)

24-8

$33-4$

$33-5$

$33-6$

33-7

TREASE, HAROLD $(x-7)$ 49-11

TREHER, ELIZABETH N. (INC-7) 50-105

TRELA, WALTER J. (DIR-O) 49-107

TRELA, WALTER J. (P-DO) 48-121 49-106 49-108

TRELA, WALTER J. (P-14) 49-109

TRKULA, MITCHELL (CHM-2) 2-3

$5-34$

47-46
TROCKI, LINDA K. (S-2) 25-11

TRUHLAR, DONALD G.

5-14

5-83

TRUJILLO, FAUSTIN (H-4)

31-27

31-28

31-43

TRUJILLO, FAUSTIN (IT-1)

$53-45$

53-59

TRUJILLO, GEORGE (LS-6)

$42-3$

TRUNILLO, PETER B. (P-9) 44-38

TRULLINGER, STEVEN EDWARD 51-15

TRUOL, $P$.

50-67

TRURAN; JAMES W.

48-84

48-114

TSCHUDY, R.H.

16-42

TSU-HSUN, SUN (MP-10) 50-21

TSURUTANI, B.T.

48-21

48-25

48-41

48-73

48-87

48-109

TUCKER, EVAF. $(W X-4)$ 26-6

TURNER, LEAF (CTR-6) 56-2

56-11

TURNER, ROBERT D. (CTR-9)

$37-4$

TURPIN, S. E.

39-65

50-21

TUSTANOFF, E. 2-28 


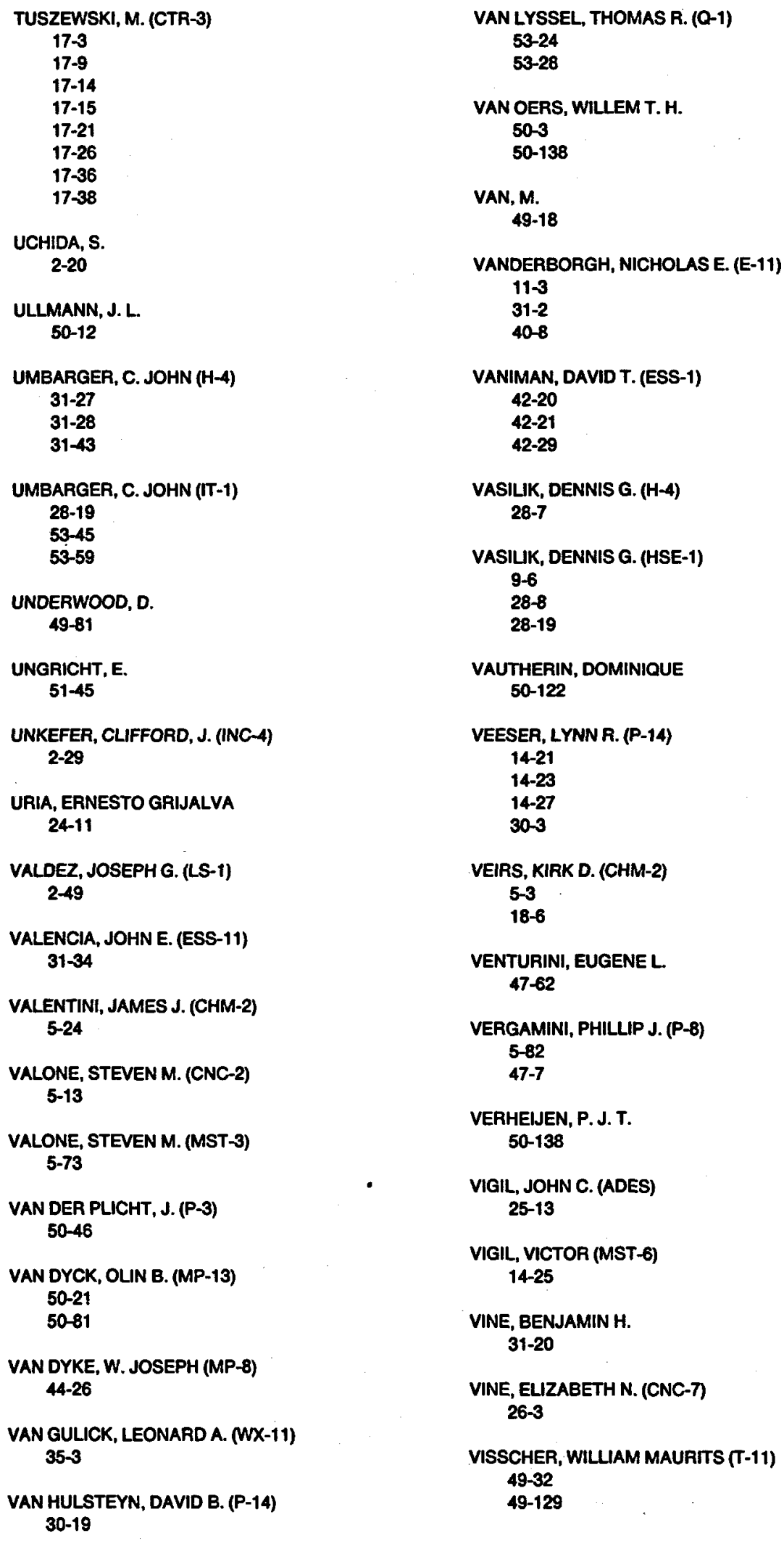

VISWANATHAN, VRIDDHACHALAM K. (P-5) 49-66 VOELZ, GEORGE L. (HSE-DO)
$28-3$

28-21

VOGEL, HERBERT F. (CTR-4) 37-9 56-10

VOGT, GERALD J. (MST-3) 38-35

VOIGT, ROBERT

$$
39-46
$$

VON ROSSEN, P.

$$
50-3
$$

VORTHMAN, JOHN E. (M-1)

$$
\text { 3-15 }
$$

VOTER, ARTHUR F. (CHM-2)

$$
\text { 5-72 }
$$$$
\text { 5-81 }
$$

VUATAZ, FRANCOIS (ESS-2)

21-5

21-7

21-10

22-3

WACKERLE, JERRY D. (M-1) 3-2

WADLINGER, E. ALAN (AT-2)

$$
\text { 44-17 }
$$

44-23

WADT, WILLARD R. (T-12)

$$
\text { 47-66 }
$$

WAECHTER, DAVID A. (E-1)

$28-8$
$28-24$
$31-32$

WAECHTER, DAVID A. (E-7) 28-7

31-27

31-28

WAECHTER, DAVID A. (E-8) 53-45

WAGNER, J. B.

50-15

WAGNER, $R$. 49-81

WAGNER, RAYMOND P. (MST-12) 53-6 


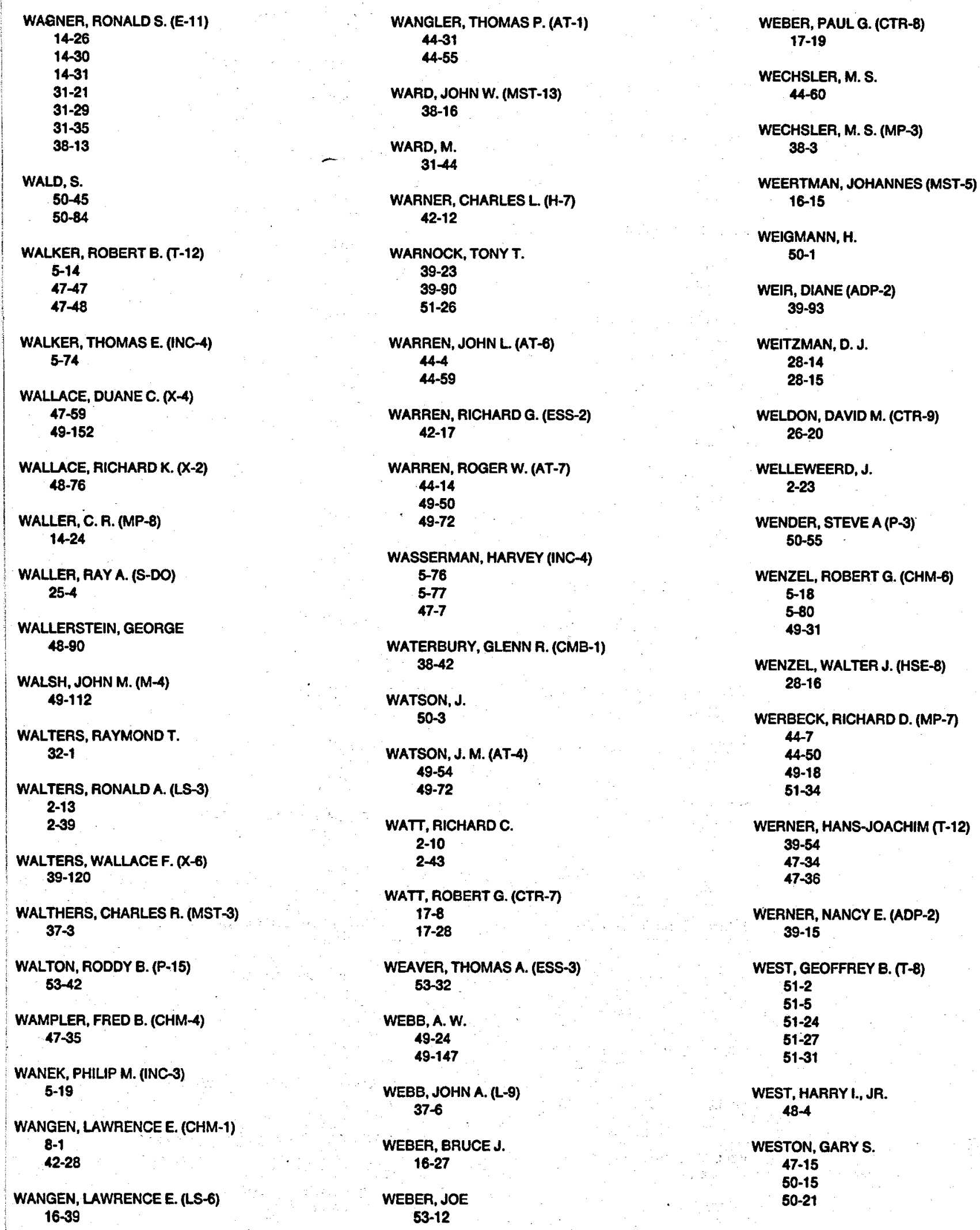

WANGLER, THOMAS P. (AT-1)
44-31 44-55

WARD, JOHN W. (MST-13) 38-16

WARD, $M$. $31-44$

WARNER, CHARLES L. (H-7) 42-12

WARNOCK, TONYT. 39-23

39-90

51-26

WARREN, JOHN L (AT-6)

44-4

44-59

WARREN, RICHARD G. (ESS-2) 42-17

WARREN, ROGER W. (AT-7) 44-14 49-50

49-72

WASSERMAN, HARVEY (INC-4) 5-76

5-77

47-7

WATERBURY, GLENN R. (CMB-1) 38-42

WATSON, J. 50-3

WATSON, J. M. (AT-4) 49-54 49.72

WATT, RICHARD C.

2-10

2-43

WATT, ROBERT G. (CTR-T) $17-8$

17-28

WEAVER, THOMAS A. (ESS-3) 53-32

WEBB, A. W. 49-24 49-147

WEBB, JOHN A. (L-9) 37-6

WEBER, BRUCE J. 16-27

WEBER, JOE

53-12 
WEWERKA, EUGENE M. (MST-DO) 40-17

WHALEN, PAUL P. $(X-D O)$

9-3

9-7

49-6

WHALEY, THOMAS W. (LS-5) 2-46

WHARTON, W. R. (LS-4)

2-41

WHEATLEY, JOHN C. (P-10)

14-24

49-17

49-91

49-155

WHEELER, GEORGE L. (LS-DO) 2-20

5-37

WHEELER, MARCIA A. (LS-DO) $2-36$

WHEELER, MERLIN L. (LS-6) 42-3

WHITAKER, D. 31-30

WHITAKER, RODNEY W. (ESS-5) 16-22

48-93

WHITCOMB, BRUCE M. 31-30

WHITE, GARY C. (LS-6)

16-27

$16-43$

WHITEHEAD, MICHAEL C. (CHM-6) 49-50

WHITEMAN, DAVID E. (S-1) 24-17

WHITFILL, JAMES A. (E-5) $37-4$

WHITTEN, $C$. 50-15.

WHITTET, D.C. B.

48-82

WHITTY, WILLIAM J. (Q-4) 53-15

53-16

WIEGEL, FREDERIK $W$. 39-92

WIENKE, BRUCE R. (C-10) 39-8
WIENKE, BRUCE R. (C-3)

39-41

$39-48$

WIGGS, LAURIE D. (HSE-14) 28-26

WILBUR, D. SCOTT (INC-3)

5-20

5-22

5-68

WILDER, MARK E. (LS-4)

2-13

2-14

2-23

WILHELMY, JERRY B. (INC-11)

$50-45$

50-46

50-84

50-105

WILKE, MARK D. (P-15) 14-62

WILKERSON, J.F. (P-3) 50-55

WILKINSON, GREGG S. (H-14) 28-25

WILKINSON, GREGG S. (HSE-14) 28-3

WILLBANKS, EMILY W. (C-8) 39-78

WILLCUTT, GORDON J., JR. (Q-7) 33-22

WILLERTON, DONALD L. (C-3) 39-24

WILLI, OSWALD (P-1)

$30-5$

30-15

WILLIAMS, ARTHUR (P-8) 50-64

WILLIAMS, ARTHUR H. (P-4) 30-7

WILLIAMS, DONNA S. (S-2) 12-3 39-121

WILLIAMS, HARRY EUGENE (MP-11) 44-10

WILLIAMS, HERBERT T. (INC-5) 24-9

WILLIAMS, JAMES M. (ADPA) 26-19
WILLIAMS, JOEL M. (LS-6) 42-28

WILLIAMS, JOEL M. (MST-6)

39-38

42-22

42-23

WILLIAMS, KEN A. (0-8) 33-20

WILLIAMS, MICHAEL D. (S-4) 16-2

16-3

16-31

16-37

WILLIAMS, ROBERT

49-18

WILLIAMS, ROBERT A. (MP-4) 51-34

WILLIAMSON, KENNETH D., JR. (S-4) 22-2

WILLIS, JEFFREY O. (MST-5)

38-18

38-23

38-32

38-41

49-103

49-156

WILLIS, WALTER LEE (AP-1)

14-34

14-35

WILSON, JOHN

49-18

WILSON, JULIE S. (LS-1)

2-44

2-48

WILSON, MAHLON T. (AT-DO) 51-39

WILSON, S. L. 51-34

WILSON, TIMOTHY L. (Q-6) 39-37

WING, G. MILTON (C-3) $51-47$

WING, J. E. (J-15) 33-1

WINKLER, CAROLYN M. (WX-11) 39-91

WINKLER, KARL-HEINZ ADOLF (X-DOT) 39-3

WINKLER, MAX A. (M-5) 49-21 


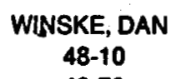

WINSKE, DAN $(X-1)$

48-56

49-92

. WINSTON, JOHN G. (E-7)

49-50

49-72

WIPF, STEFAN L. (AT-6) 44-18

WIPF, STEFAN L. (P-DO)

$$
\text { 44-12 }
$$

WIRE, MIKE S. (MST-5)

38-19

38-31

38-32

38-38

49-156

WIRZBA, A.

50-22

WISNIEWSKI, W. Z.

48-71

WOFSY, CARLA

2-18

WOHLETZ, KENNETH H. (ESS-1)

16-18

16-26

22-3

WOLCOTT, JOHN H. (ESS-7) 48-60

WOLF, MICHAEL ALLEN (H-4)

31-27

$31-28$

$31-43$

WOLF, MICHAEL ALLEN (IT-1) 53-45 53-59

WOLF, RICHARD J. (C-6) $39-99$

WOLF, S. A.

49-24

49-67

49-128

49-147

WOLF, T.

5-60

WOLFE, MICHAEL L. 16-27

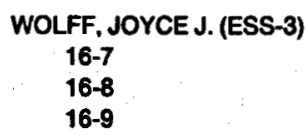

WOLFF, WALTER P. (NSP/TO) 26-21

WOLFSBERG, KURT (INC-7) 42-21

$50-63$

WOOD, B. E. (P-15)

50-69

WOOD, ELIZABETH J. (P-8) 50-72

WOOD, S. A.

50-41

50-93

WOOD, WARREN T. (MST-14) 35-1

WOOD, WILLIAM W. (T-11) 49-85

WOODFIN, GREG L. (P-5) 49-66

WOODRUFF, SUSAN B. (Q-8) 33-3

WOODRUFF, WILLIAM H. 47-63

WOOLUM, D, S. 48.69

WOOSLEY, S.E. 48-76

WORRALL, D.M. 48-71

WOUTERS, JAN M. (INC-11) 39-65

WRATHALL, JAY 48-52

WRAY, WILLIAM O. (Q-11) 29-16

WRAY, WILLIAM O. (Q-13) 29-6

WRIGHT, BRADFORD L. (CTR-5)

18-1 18-10

WRIGHT, S.C.

49-18

51-34

WRIGHT, WALTER J. (MST-5) 14-22
Wu, c. s.

48-10

WU, J.

50-94

WUDL, FRANK

49-128

WUHL, HELMUT

38-39 WURDEN, GLEN A. (CTR-8)
$17-30$

WYSIN, GARY 51-35

XU, SHEN-WU 50-21

YAMADA, TETSUJI (ESS-5) 16-31

YAMAGUCHI, KENJIRO

29-3

29-5

YAMAZAKI, AKIO (LS-DO)

2-20

2-36

YAOUANC, ALAIN (MP-3) 38-11

YATES, GEORGE J. (P-15) 31-20

31-26

31-42

YATES, MARY ANNE (P-4) 30-19

YAVORNIK, EDWARD J. (CTR-4) 18-14

YEAMANS, MARILYN K. (S-2) 25-11

YEH, XIAN LI 50-64

YOKOSAWA, A. 49-81

YORK, ROBBIE LYNN (MP-11) 14-11

44-39

44-63

49-47

$49-48$

YOUNG, AINSLIE T., JR. (MST-6) 38-22

YOUNG, CARLTON S. (P-14)

31-16 


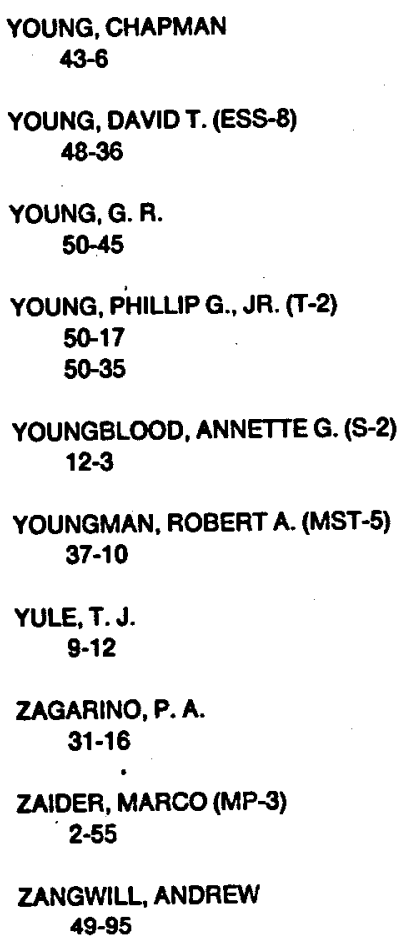

ZARDECKI, ANDREW (T-DO)

$39-4$

49-88

49-153

ZEIDMAN, B.

$51-45$

ZEIRI, YEHUDA

47-8

ZEMACH, CHARLES (T-DOT) 51-26

ZHOU, G. C.

48-10

ZIEGEL, E. R.

5-60

ZIMM, C. B. (P-10)

49-73

ZIMMERMANN, EUGENEL L (P-1)

14-21

14-23

ZUMBRO, J.D.

50-74

50-75

50-102
ZWEIG, GEORGE (T-DOT)

51-27

ZWICKL, RONALD D. (ESS-8)

48-17

48-21

48-25

48-31

48-32

48-35

48-37

48-38

48-41

48-72

48-74

48-87

48-68

48-109

ZYSKIND, JOHN

50-92

ZYVOLOSKI, GEORGE A (ESS-4)

27-1 
NUMERIC INDEX

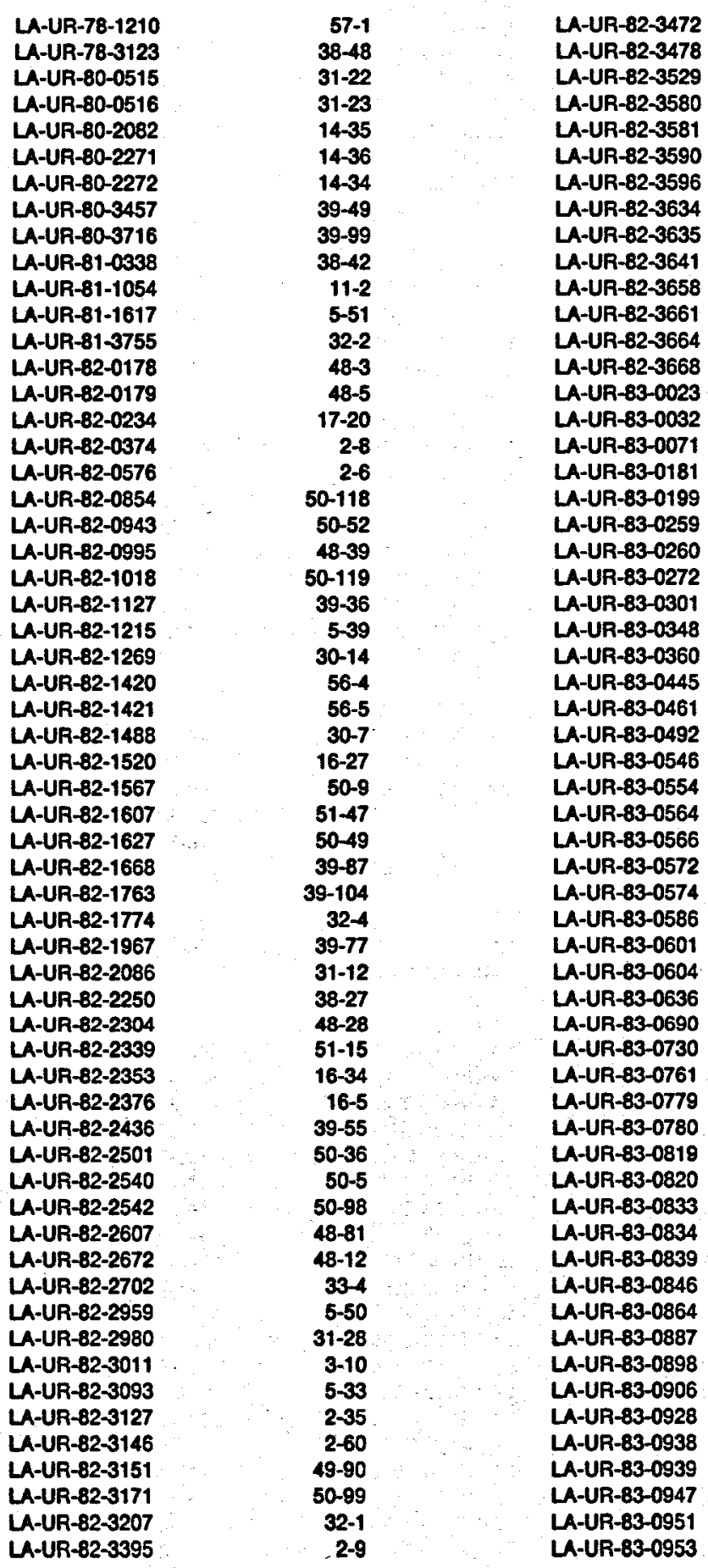

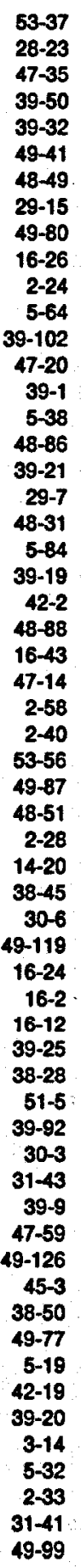

LA-UR-83-0962

LA-UR-63-0990

LA-UR-83-1001

LA-UR-83-1002

LA-UR-83-1020

LA-UR-83-1022

LA-UR-83-1028

LA-UR-83-1029

LA-UR-83-1030

LA-UR-83-1036

LA-UR-83-1042

LA-UR-83-1057

LA-UR-83-1065

LA-UR-83-1066

LA-UR-83-1067

LA-UR-83-1070

LA-UR-83-1071

LA-UR-83-1072

LA-UR-83-1079

LA-UR-83-1081

LA-UR-83-1089

LA-UR-83-1090

LA-UR-63-1128

LA-UR-83-1132

LA-UR-83-1172

LA-UR-83-1177

LA-UR-63-1207

LA-UR-83-1209

LA-UR-B3-1211

LA-UR-83-1227

LA-UR-83-1245

LA-UR-63-1252

LA-UR-83-1262

LA-UR-83-1298

LA-UR-83-1299

LA-UR-83-1308

LA-UR-83-1310

LA-UR-83-1314

LA-UR-83-1316

LA-UR-83-1333

LA-UR-83-1361

LA-UR-83-1365

LA-UR-83-1379

LA-UR-83-1384

UA-UR-83-1390

LA-UR-83-1392

LA-UR-83-1397

LA-UR-83-1432

LA-UR-83-1442

LA-UR-83-1466

LA-UR-83-1473

LA-UR-83-1474

LA-UR-83-1478

LA-UR-83-1495

LA-UR-83-1499

LA-UR-83-1500

LA-UR-83-1511

LA-UR-83-1529

LA-UR-83-1531 $\begin{array}{r}2-19 \\ 29-12 \\ 5-67 \\ 16-45 \\ 49-143 \\ 48-35 \\ 47-37 \\ 47-58 \\ 50-4 \\ 48-52 \\ 48-111 \\ 5-13 \\ 31-32 \\ 31-27 \\ 28-7 \\ 49-158 \\ 44-60 \\ 39-14 \\ 53-30 \\ 48-72 \\ 31-25 \\ 31-13 \\ 50-17 \\ 49-144 \\ 48-73 \\ 31-5 \\ 39-11 \\ 29-10 \\ 49-159 \\ 5-73 \\ 5-1 \\ 49-155 \\ 51-29 \\ 49-102 \\ 47-45 \\ 2-30 \\ 51-1 \\ 14-57 \\ 5-57 \\ 16-23 \\ 47-23 \\ 48-1 \\ 38-7 \\ 50-53 \\ 39-105 \\ 39-70 \\ 50-16 \\ 2-23 \\ 31-38 \\ 44-10 \\ 38-44 \\ 2-20 \\ 14-14 \\ 48-102 \\ 50-73 \\ 39-23 \\ 49-132 \\ 49-96 \\ 2-14 \\ \hline\end{array}$ 


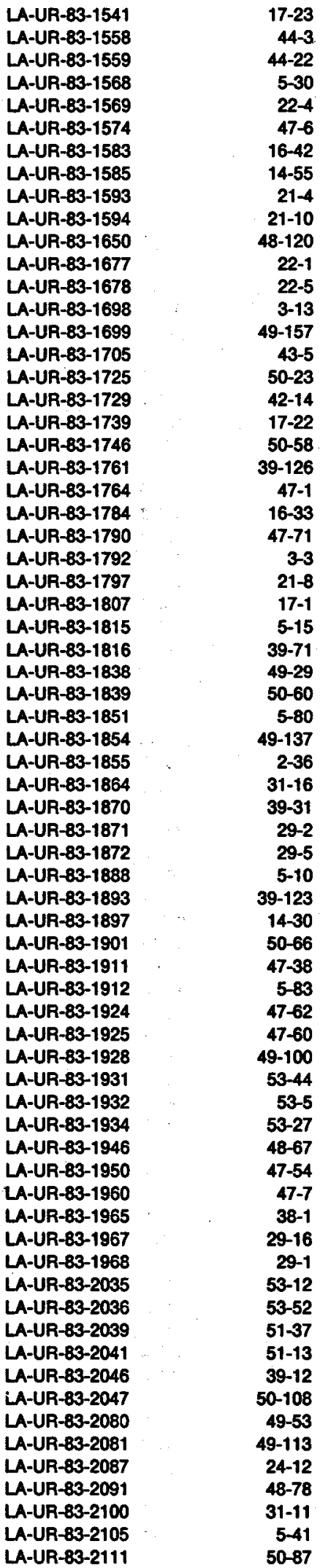

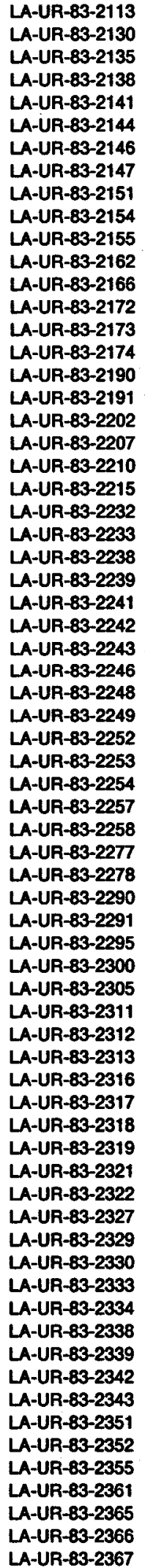

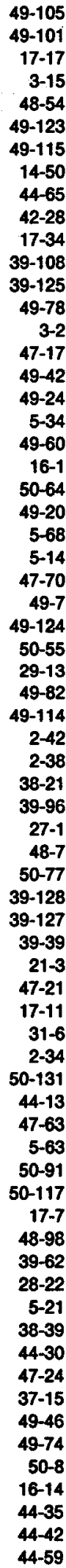

LA-UR-83-2368

LA-UR-83-2370

LA-UR-83-2375

LA-UR-83-2378

LA-UR-83-2379

LA-UR-83-2383

LA-UR-83-2386

LA-UR-83-2390

LA-UR-83-2395

LA-UR-83-2396

LA-UR-83-2398

LA-UR-83-2399

LA-UR-83-2400

LA-UR-83-2404

LA-UR-83-2406

LA-UR-83-2408

LA-UR-83-2411

LA-UR-83-2416

LA-UR-83-2424

LA-UR-83-2425

LA-UR-83-2426

LA-UR-83-2430

LA-UR-83-2431

LA-UR-83-2432

LA-UR-83-2438

LA-UR-83-2440

LA-UR-83-2443

LA-UR-83-2445

LA-UR-83-2449

LA-UR-83-2451

LA-UR-83-2456

LA-UR-83-2457

LA-UR-83-2460

LA-UR-83-2465

LA-UR-83-2466

LA-UR-83-2467

LA-UR-83-2476

LA-UR-83-2477

LA-UR-83-2481

LA-UR-83-2485

LA-UR-83-2486

LA-UR-83-2493

LA-UR-83-2497

LA-UR-83-2503

LA-UR-83-2507

LA-UR-83-2514

LA-UR-83-2515

LA-UR-83-2516

LA-UR-83-2529

LA-UR-83-2531

LA-UR-83-2540

LA-UR-83-2541

LA-UR-83-2545

LA-UR-83-2548

LA-UR-83-2549

LA-UR-83-2562

LA-UR-83-2566

LA-UR-83-2567

LA-UR-83-2568

LA-UR-83-2569

LA-UR-83-2570

LA-UR-83-2571

LA-UR-83-2572

LA-UR-83-2578

LA-UR-83-2579

LA-UR-83-2580

LA-UR-83-2583

LA-UR-63-2586

LA-UR-83-2587

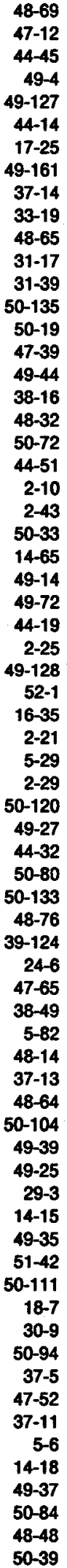




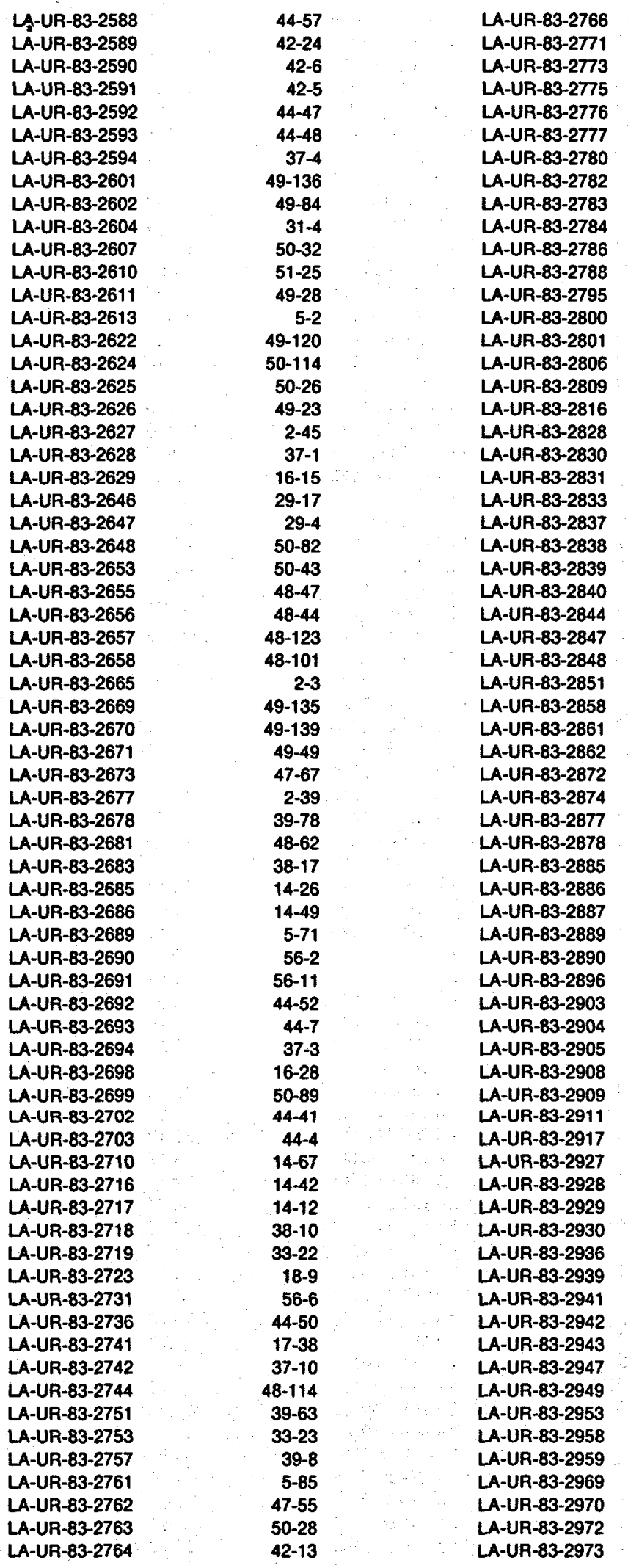

\begin{tabular}{|c|c|c|}
\hline $48-61$ & LA-UR-83-2979 & $39-89$ \\
\hline 53-3 & LA-UR-83-2980 & $39-76$ \\
\hline $2-37$ & LA-UR-83-2982 & $38-46$ \\
\hline $38-11$ & LA-UR-83-2983 & $39-40$ \\
\hline $48-66$ & LA-UR-83-2989 & 30-19 \\
\hline $48-113$ & LA-UR-83-2997 & $48-68$ \\
\hline 33-17 & LA-UR-83-2998 & $48-42$ \\
\hline 49-71 & LA-UR-83-3007 & $47-28$ \\
\hline $37-8$ & LA-UR-83-3008 & $51-20$ \\
\hline $14-25$ & LA-UR-83-3009 & $14-17$ \\
\hline 33-14 & LA-UR-83-3010 & $47-46$ \\
\hline $48-74$ & LA-UR-83-3011 & $33-21$ \\
\hline $31-8$ & LA-UR-83-3012 & $47-49$ \\
\hline $49-43$ & LA-UR-83-3014 & $47-16$ \\
\hline 5-59 & LA-UR-83-3015 & $47-33$ \\
\hline $33-25$ & LA-UR-83-3017 & $48-83$ \\
\hline $17-10$ & LA-UR-83-3019 & $38-41$ \\
\hline 33-16 & LA-UR-83-3024 & $42-12$ \\
\hline $50-44$ & LA-UR-83-3026 & 33-13 \\
\hline $49-67$ & LA-UR-83-3028 & $11-1$ \\
\hline 14-21 & LA-UR-83-3029 & $47-13$ \\
\hline $48-45$ & LA-UR-83-3032 & $53-22$ \\
\hline $49-107$ & LA-UR-83-3036 & $39-41$ \\
\hline $5-61$ & LA-UR-83-3041 & $43-2$ \\
\hline $50-134$ & LA-UR-83-3042 & $31-26$ \\
\hline $30-13$ & LA-UR-83-3045 & $38-34$ \\
\hline 48-55 & LA-UR-83-3049 & $39-79$ \\
\hline $3-11$ & LA-UR-83-3052 & $39-16$ \\
\hline $39-84$ & LA-UR-83-3056 & 38-37 \\
\hline $50-112$ & LA-UR-83-3058 & $50-2$ \\
\hline $47-40$ & LA-UR-83-3060 & $31-42$ \\
\hline 49-85 & LA-UR-83-3061 & 50-54 \\
\hline 39-94 & LA-UR-83-3062 & $33-15$ \\
\hline $38-13$ & LA-UR-83-3064 & $38-18$ \\
\hline $47-30$ & LA-UR-83-3065 & $48-118$ \\
\hline $28-4$ & LA-UR-83-3067 & 49-12 \\
\hline $49-59$ & LA-UR-83-3068 & $51-41$ \\
\hline $39-60$ & LA-UR-83-3069 & $17-30$ \\
\hline $39-42$ & LA-UR-B3-3071 & $27-2$ \\
\hline 48-36 & LA-UR-83-3072 & $27-3$ \\
\hline $48-109$ & LA-UR-83-3074 & $50-106$ \\
\hline $49-73$ & LA-UR-83-3075 & $48-2$ \\
\hline $48-77$ & LA-UR-83-3077 & $5-79$ \\
\hline 47-5 & LA-UR-83-3088 & $48-112$ \\
\hline $47-56$ & LA-UR-83-3091 & $49-129$ \\
\hline $5-11$ & LA-UR-83-3093 & $2-1$ \\
\hline $49-18$ & LA-UR-83-3095 & $47-2$ \\
\hline $\begin{array}{l}53-31 \\
40-10\end{array}$ & $\begin{array}{l}\text { LA-UR-83-3096 } \\
\text { LA-UR-83-3097 }\end{array}$ & $\begin{array}{l}51-22 \\
50-95\end{array}$ \\
\hline $5-74$ & LA-UR-83-3098 & $49-48$ \\
\hline $50-24$ & LA-UR-83-3099 & $47-27$ \\
\hline $49-147$ & LA-UR-83-3101 & 48-18 \\
\hline $49-125$ & LA-UR-83-3110 & $2-46$ \\
\hline $48-107$ & LA-UR-83-3116 & 33-12 \\
\hline $48-93$ & LA-UR-83-3120 & $48-80$ \\
\hline 9-7 & LA-UR-83-3127 & $33-24$ \\
\hline $53-59$ & LA-UR-B3-3136 & $5-55$ \\
\hline $53-45$ & LA-UR-83-3143 & $48-116$ \\
\hline $50-125$ & LA-UR-83-3149 & $16-16$ \\
\hline $38-40$ & LA-UR-83-3150 & 48-59 \\
\hline $33-20$ & LA-UR-83-3156 & $2-55$ \\
\hline $47-42$ & LA-UR-83-3157 & $5-76$ \\
\hline $28-8$ & LA-UR-83-3158 & $5-77$ \\
\hline $28-24$ & LA-UR-B3-3169 & 38-35 \\
\hline $48-100$ & LA-UR-83-3189 & $17-9$ \\
\hline $5-12$ & LA-UR-83-3194 & $50-137$ \\
\hline $17-31$ & LA-UR-83-3199 & 53-1 \\
\hline $51-3$ & LA-UR-83-3200 & $50-124$ \\
\hline
\end{tabular}




\begin{tabular}{|c|c|}
\hline $\begin{array}{l}\text { LA-UR-83-3204 } \\
\text { LA-UR-83-3205 }\end{array}$ & $\begin{array}{r}50-22 \\
39-2\end{array}$ \\
\hline LA-UR-83-3215 & $48-15$ \\
\hline LA-UR-83-3216 & $48-106$ \\
\hline LA-UR-83-3217 & $48-108$ \\
\hline LA-UR-83-3229 & $30-20$ \\
\hline LA-UR-83-3239 & $48-85$ \\
\hline LA-UR-83-3241 & $2-26$ \\
\hline LA-UR-83-3242 & $17-28$ \\
\hline LA-UR-83-3243 & $39-81$ \\
\hline LA-UR-83-3246 & $44-58$ \\
\hline LA-UR-83-3250 & $50-132$ \\
\hline LA-UR-83-3252 & $53-41$ \\
\hline LA-UR-83-3254 & $44-36$ \\
\hline LA-UR-63-3257 & $5-4$ \\
\hline LA-UR-63-3258 & $5-75$ \\
\hline LA-UR-83-3265 & $53-4$ \\
\hline LA-UR-83-3266 & $53-21$ \\
\hline LA-UR-83-3273 & 44-11 \\
\hline LA-UR-83-3277 & $2-49$ \\
\hline LA-UR-83-3278 & $49-55$ \\
\hline LA-UR-83-3288 & 44-28 \\
\hline LA-UR-83-3290 & $39-93$ \\
\hline LA-UR-83-3292 & $50-15$ \\
\hline LA-UR-83-3293 & $38-2$ \\
\hline LA-UR-83-3295 & $5-16$ \\
\hline LA-UR-83-3298 & $53-20$ \\
\hline LA-UR-83-3299 & $53-11$ \\
\hline LA-UR-83-3302 & $39-107$ \\
\hline LA-UR-83-3313 & $50-86$ \\
\hline LA-UR-83-3320 & $53-46$ \\
\hline LA-UR-83-3325 & $53-49$ \\
\hline LA-UR-83-3329 & $53-36$ \\
\hline LA-UR-83-3340 & $39-15$ \\
\hline LA-UR-83-3341 & $42-15$ \\
\hline LA-UR-83-3344 & $53-43$ \\
\hline LA-UR-83-3345 & 48-17 \\
\hline LA-UR-83-3347 & $47-47$ \\
\hline LA-UR-83-3349 & $9-8$ \\
\hline LA-UR-83-3359 & $51-40$ \\
\hline LA-UR-83-3361 & $48-89$ \\
\hline LA-UR-83-3368 & $48-99$ \\
\hline LA-UR-83-3379 & $47-53$ \\
\hline LA-UR-83-3381 & 53-42 \\
\hline LA-UF-83-3382 & $53-28$ \\
\hline LA-UR-83-3383 & $53-39$ \\
\hline LA-UR-83-3384 & $53-33$ \\
\hline LA-UR-83-3385 & $42-10$ \\
\hline LA-UR-83-3386 & $53-19$ \\
\hline LA-UR-83-3391 & $2-17$ \\
\hline LA-UR-63-3392 & $53-29$ \\
\hline LA-UR-83-3393 & $39-47$ \\
\hline LA-UR-83-3394 & $39-29$ \\
\hline LA-UR-83-3396 & $39-52$ \\
\hline LA-UR-83-3397 & $39-24$ \\
\hline LA-UR-83-3398 & $31-10$ \\
\hline LA-UR-83-3402 & $42-26$ \\
\hline LA-UR-83-3403 & $53-38$ \\
\hline LA-UR-83-3404 & $24-9$ \\
\hline LA-UR-83-3405 & 24-4 \\
\hline LA-UR-83-3409 & $31-37$ \\
\hline LA-UR-83-3410 & $39-59$ \\
\hline LA-UR-63-3415 & $18-16$ \\
\hline LA-UR-83-3419 & $50-105$ \\
\hline LA-UR-83-3421 & $39-98$ \\
\hline LA-UR-83-3422 & $14-22$ \\
\hline LA-UR-83-3423 & $39-33$ \\
\hline LA-UR-83-3424 & $49-30$ \\
\hline
\end{tabular}

LA-UR-83-3428

LA-UR-83-3429

LA-UR-83-3439

LA-UR-83-3440

LA-UR-83-3441

LA-UR-83-3443

LA-UR-83-3444

LA-UR-83-3451

LA-UR-83-3452

LA-UR-83-3453

LA-UR-83-3454

LA-UR-83-3455

LA-UR-83-3470

LA-UR-83-3473

LA-UR-83-3476

LA-UR-83-3477

LA-UR-83-3480

LA-UR-83-3481

LA-UR-83-3489

LA-UR-83-3490

LA-UR-83-3492

LA-UR-83-3498

LA-UR-83-3499

LA-UR-83-3502

LA-UR-83-3505

LA-UR-83-3506

LA-UR-83-3513

LA-UR-83-3514

LA-UR-83-3517

LA-UR-63-3520

LA-UR-83-3521

LA-UR-83-3523

LA-UR-83-3524

LA-UR-83-3527

LA-UR-83-3530

LA-UR-83-3531

LA-UR-83-3538

LA-UR-83-3543

LA-UR-83-3544

LA-UR-83-3545

LA-UR-83-3546

LA-UR-83-3553

LA-UR-83-3569

LA-UR-83-3573

LA-UR-83-3580

LA-UR-83-3582

LA-UR-83-3583

LA-UR-83-3585

LA-UR-83-3588

LA-UR-83-3593

LA-UR-83-3602

LA-UR-83-3604

LA-UR-83-3612

LA-UR-83-3620

LA-UR-83-3621

LA-UR-83-3622

LA-UR-83-3624

LA-UR-83-3626

LA-UR-83-3630

LA-UR-83-3634

LA-UR-83-3638

LA-UR-83-3647

LA-UR-63-3648

LA-UR-83-3651

LA-UR-83-3652

LA-UR-83-3656

LA-UR-83-3662

LA-UR-83-3683

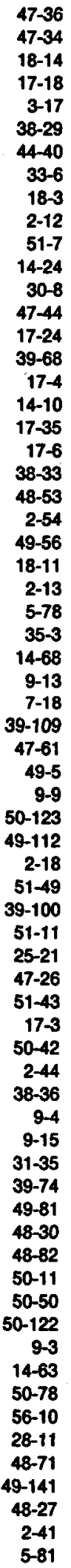

LA-UR-83-3686

LA-UR-83-3688

LA-UR-83-3691

LA-UR-83-3695

LA-UR-83-3701

LA-UR-83-3702

LA-UR-63-3704

LA-UR-83-3706

LA-UR-83-3713

LA-UR-63-3715

LA-UR-83-3716

LA-UR-83-3717

LA-UR-83-3720

LA-UR-84-0001

LA-UR-84-0006

LA-UR-84-0007

LA-UR-84-0013

LA-UR-84-0015

LA-UR-84-0016

LA-UR-84-0017

LA-UR-84-0018

LA-UR-84-0022

LA-UR-84-0031

LA-UR-84-0032

LA-UR-84-0034

LA-UR-84-0037

LA-UR-84-0047

LA-UR-84-0048

LA-UR-84-0052

LA-UR-84-0058

LA-UR-84-0059

LA-UR-84-0060

LA-UR-84-0062

LA-UR-84-0064

LA-UR-84-0073

LA-UR-84-0077

LA-UR-84-0081

LA-UR-84-0089

LA-UR-84-0096

LA-UR-84-0099

LA-UR-84-0109

LA-UR-84-0119

LA-UR-84-0120

LA-UR-84-0123

LA-UR-84-0124

LA-UR-84-0136

LA-UR-84-0138

LA-UR-84-0139

LA-UR-84-0142

LA-UR-84-0143

LA-UR-84-0144

LA-UR-84-0145

LA-UR-64-0157

LA-UR-B4-0164

LA-UR-84-0165

LA-UR-84-0168

LA-UR-84-0169

LA-UR-84-0170

LA-UR-84-0175

LA-UR-84-0178

LA-UR-84-0183

LA-UR-84-0185

LA-UR-84-0186

LA-UR-84-0191

LA-UR-84-0193

LA-UR-84-0197

LA-UR-84-0199

LA-UR-84-0200

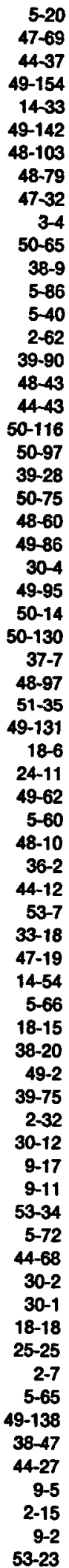




LA-UR-84-0202
LA-UR-84-0209
LA-UR-84-0216
LA-UR-84-0217
LA-UR-84-0218
LA-UR-84-0221
LA-UR-84-0224
LA-UR-84-0226
LA-UR-84-0240
LA-UR-84-0259
LA-UR-84-0264
LA-UR-84-0265
LA-UR-84-0274
LA-UR-84-0276
LA-UR-84-0304
LA-UR-84-0305
LA-UR-84-0327
LA-UR-84-0330
LA-UR-84-0339
LA-UR-84-0344
LA-UR-84-0357
LA-UR-84-0363
LA-UR-84-0384
LA-UR-84-0391
LA-UR-84-0396
LA-UR-84-0411
LA-UR-84-0419
LA-UR-84-0424
LA-UR-84-0429
LA-UR-84-0431
LA-UR-84-0433
LA-UR-84-0434
LA-UR-84-0441
LA-UR-84-0442
LA-UR-84-0443
LA-UR-84-0451
LA-UR-84-0459
LA-UR-84-0465
LA-UR-84-0483
LA-UR-84-0498
LA-UR-84-0500
LA-UR-84-0512
LA-UR-84-0515
LA-UR-84-0516
LA-UR-84-0518
LA-UR-84-0524
LA-UR-84-0535
LA-UR-84-0541
LA-UR-84-0553
LA-UR-84-0581
LA-UR-84-0583
LA-UR-84-0584
LA-UR-84-0599
LA-UR-84-0601
LA-UR-84-0622
LA-UR-84-0602
LA-UR-84-0623
LA-UR-84-0603-0625
LA-UR-84-0604
LA-UR-84-0606
LA-UR-84-0626
LA-UR-84-0629
LA-UR-84-0630
LA-UR-84-0631
LA-0620
LAR-8621

LA-UR-84-0202

LA-UR-84-0209

LA-UR-84-0216

R-84-0217

LA-UR-84-0218

LA-UR-84-0224

LA-UR-84-0226

LA-UR-84-0240

LA-UR-84-0265

-84-0274

LA-UR-84-0304

LA-UR-84-0305

LA-UR-84-0330

LA-UR-84-0339

84-0344

LA-UR-84-0363

-UR-84-0384

LA-UR-84-0396

LA-UR-64-0411

LA-UR-84-0424

LA-UR-84-0429

LA-UR-84-0431

LA-UR-84-0434

LA-UR-84-0443

LA-UR-84-0459

LA-UR-84-0465

LA-UR-84-0498

LA-UR-84-0500

LA-UR-84-0515

LA-UR-84-0516

UA-UR-84-052

LA-UR-84-0553

LA-UR-84-0581

LA-UR-84-0584

LA-UR-84-0599

A-UR-84-0602

LA-UR-84-0603

LA-UR-84-0604

LA-UR-84-0620

LA-UR-84-0621

LA-UR-84-0624

LA-UR-84-0625

LA-UR-84-0629

LA-UR-84-0631

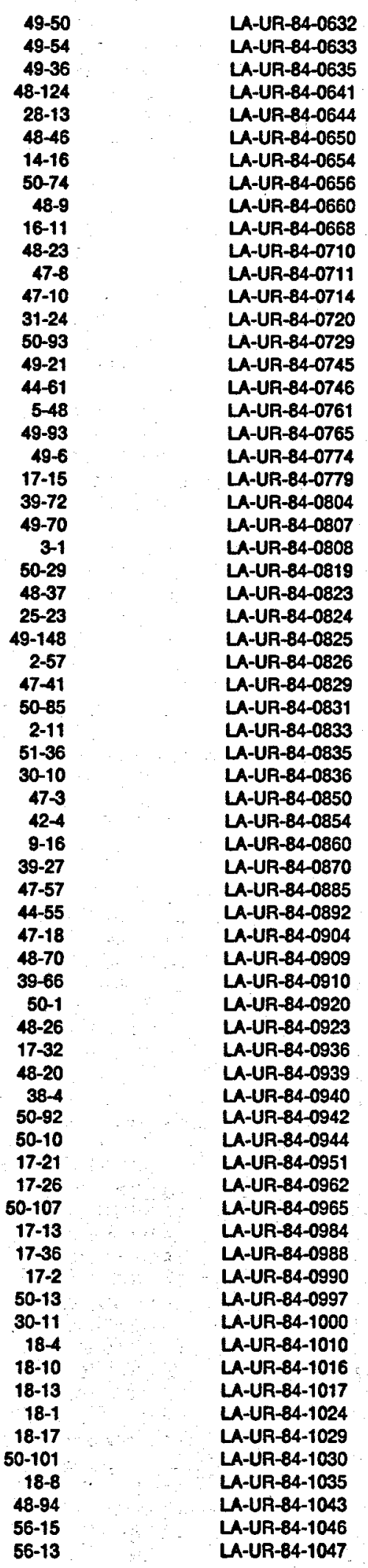

56-9

56-12

5-18

14-48

51-21

39-10

38-43

14-23

5-56

5-58

50-34

5-35

17-29

49-108

48-115

14-39

14-27

$50-88$

47-68

14-6

$50-46$

50-63

56-3

$16-36$

48-4

50-56

51-12

$51-4$

51-50

16-4

50-102

47-50

50-48

17-33

48-110

17-19

47-4

17-14

17-16

48-56

49-76

38-32

49-66

49-52

17.37

5-24

51-23

51-2

49-58

49-98

48-41

16-18

45-2

31-31

39-5

44-33

5-22

$16-30$

51-18

38-6

38-24

47-25

50-3

50-138

16-37

50-68

$50-35$

39-53
LA-UR-64-1054

LA-UR-64-1063

LA-UR-84-1065

LA-UR-84-1067

LA-UR-84-1069

LA-UR-84-1076

LA-UR-84-1085

LA-UR-84-1093

LA-UR-84-1104

LA-UR-84-1106

LA-UR-84-1110

LA-UR-84-1120

LA-UR-84-1121

LA-UR-84-1122

LA-UR-84-1124

LA-UR-84-1127

LA-UR-84-1130

LA-UR-84-1131

LA-UR-84-1139

LA-UR-84-1144

LA-UR-84-1154

LA-UR-84-1155

LA-UR-84-1156

LA-UR-84-1164

LA-UR-84-1165

LA-UR-84-1170

LA-UR-84-1172

LA-UR-84-1173

LA-UR-84-1186

LA-UR-84-1187

LA-UR-84-1190

LA-UR-84-1191

LA-UR-84-1199

LA-UR-84-1207

LA-UR-84-1208

LA-UR-84-1211

LA-UR-84-1218

LA-UR-84-1232

LA-UR-84-1235

LA-UR-84-1238

LA-UR-84-1249

LA-UR-84-1250

LA-UR-84-1251

LA-UR-84-1272

LA-UR-84-1276

LA-UR-84-1278

LA-UR-84-1279

LA-UR-84-1280

LA-UR-84-1281

LA-UR-84-1282

LA-UR-84-1289

LA-UR-84-1293

LA-UR-84-1294

LA-UR-84-1306

LA-UR-84-1307

LA-UR-84-1308

LA-UR-84-1311

LA-UR-84-1314

LA-UR-84-1319

LA-UR-84-1321

LA-UR-84-1323

LA-UR-84-1324

LA-UR-84-1325

LA-UR-84-1327

LA-UR-84-1332

LA-UR-84-1338

LA-UR-84-1342

LA-UR-84-1343
5-36

30-18

$51-6$

48-104

43-4

17-12

51-38

$56-8$

49-8

21-2

48-50

39-17

2-50

2-51

38-3

49-75

31-29

50-37

13-1

5-7

30-5

48-29

48-117

5-46

49-92

47-31

48-13

50-103

22-8

$31-21$

49-63

38-12

48-33

50-62

21.5

49-97

47-11

44-63

47-48

40-7

$17-5$

48-38

47-64

2-16

38-31

44-23

44-17

44-9

44-56

39-112

26-4

50-126

29-11

44-64

44-15

44-44

51-10

16-41

33-5

39-82

50-51

39-26

5-5

49-122

25-17

29-6

44-31

39-97 


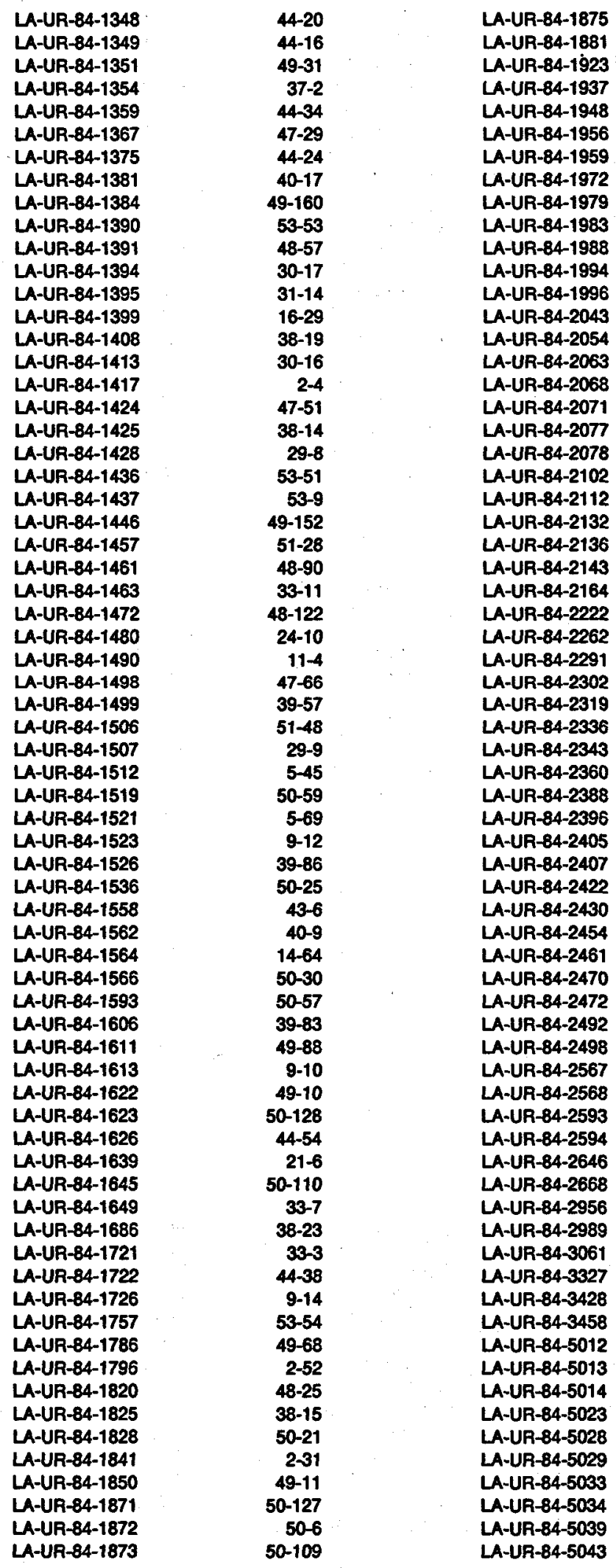

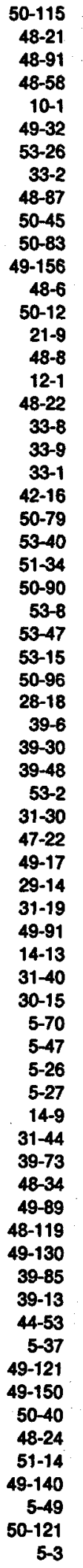

LA-UR-84-5052

LA-UR-84-5053

LA-UR-84-5054

LA-UR-84-5055

LA-UR-84-5057

LA-UR-84-5058

LA-UR-84-5059

LA-UR-84-5061

LA-UR-84-5062

LA-UR-84-5063

LA-UR-84-5064

LA-UR-84-5065

LA-UR-84-5066

LA-UR-84-5067

LA-UR-84-5068

LA-UR-84-5069

LA-UR-84-5070

LA-UR-84-5071

LA-UR-84-5072

LA-UR-84-5073

LA-UR-84-5074

LA-UR-84-5075

LA-UR-84-5076

LA-UR-84-5077

LA-UR-84-5078

LA-UR-84-5079

LA-UR-84-5080

LA-UR-84-5081

LA-UR-84-5082

LA-UR-84-5083

LA-UR-84-5084

LA-UR-84-5085

LA-UR-84-5086

LA-UR-84-5087

LA-UR-84-5088

LA-UR-84-5089

LA-UR-84-5090

LA-UR-84-5091

LA-UR-84-5092

LA-UR-84-5093

LA-UR-84-5094

LA-UR-84-5095

LA-UR-84-5096

LA-UR-84-5097

LA-UR-84-5098

LA-UR-84-5099

LA-UR-84-5100

LA-UR-84-5101

LA-UR-84-5102

LA-UR-84-5103

LA-UR-84-5104

LA-UR-84-5105

LA-UR-84-5106

LA-UR-84-5107

LA-UR-84-5108

LA-UR-84-5110

LA-UR-85-5000

LA-UR-85-5001

LA-UR-85-5002

LA-UR-85-5003

LA-UR-85-5004

LA-UR-85-5006

LA-UR-85-5008

LA-UR-85-5009

LA-UR-85-5011

LA-UR-85-5012

LA-UR-85-5013

LA-UR-85-5014

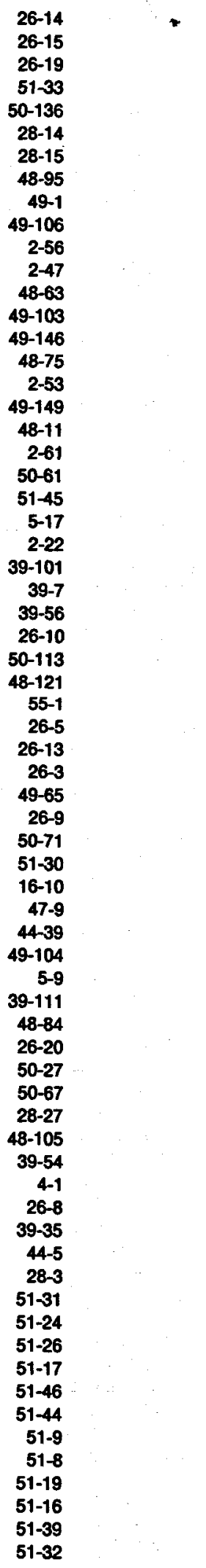




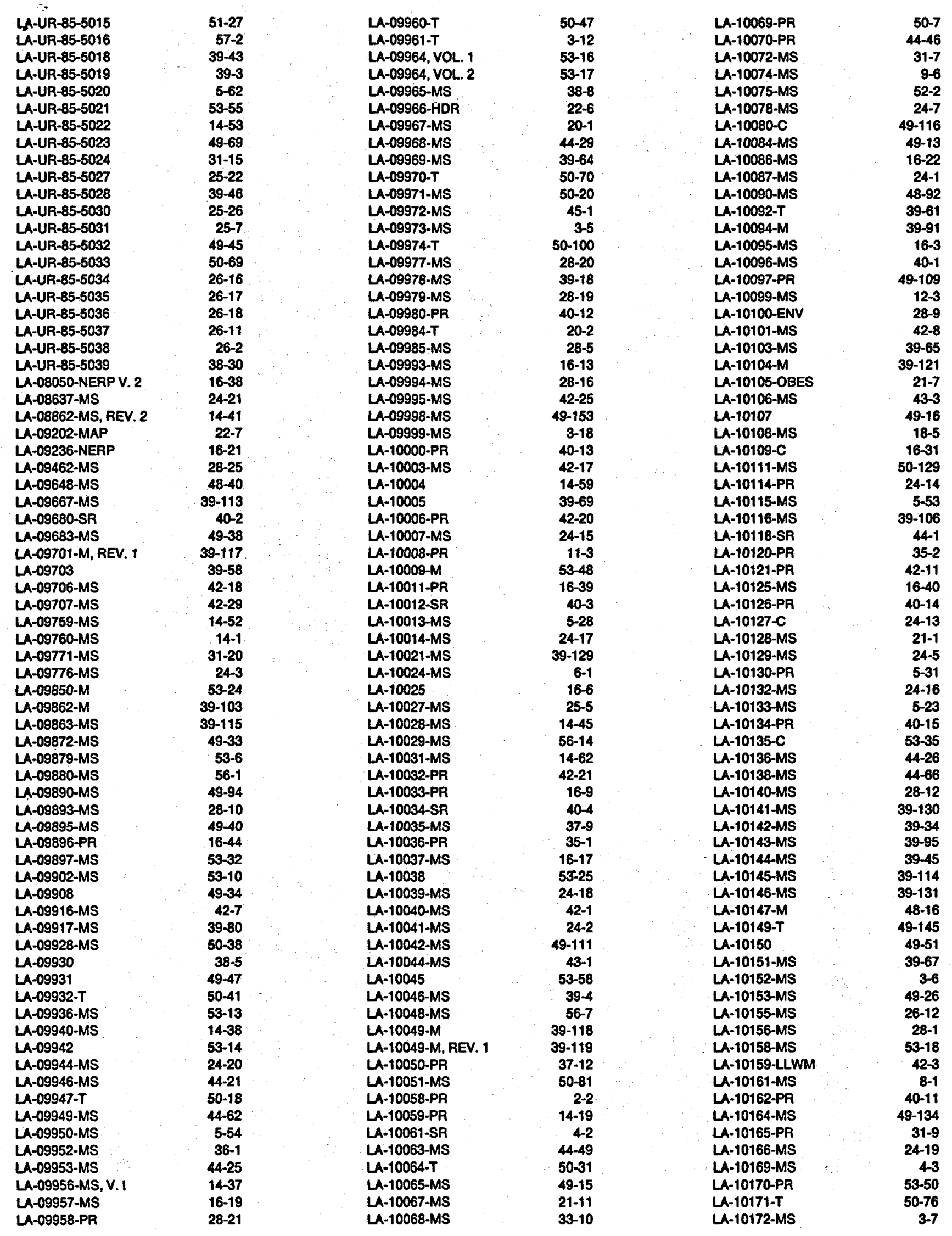




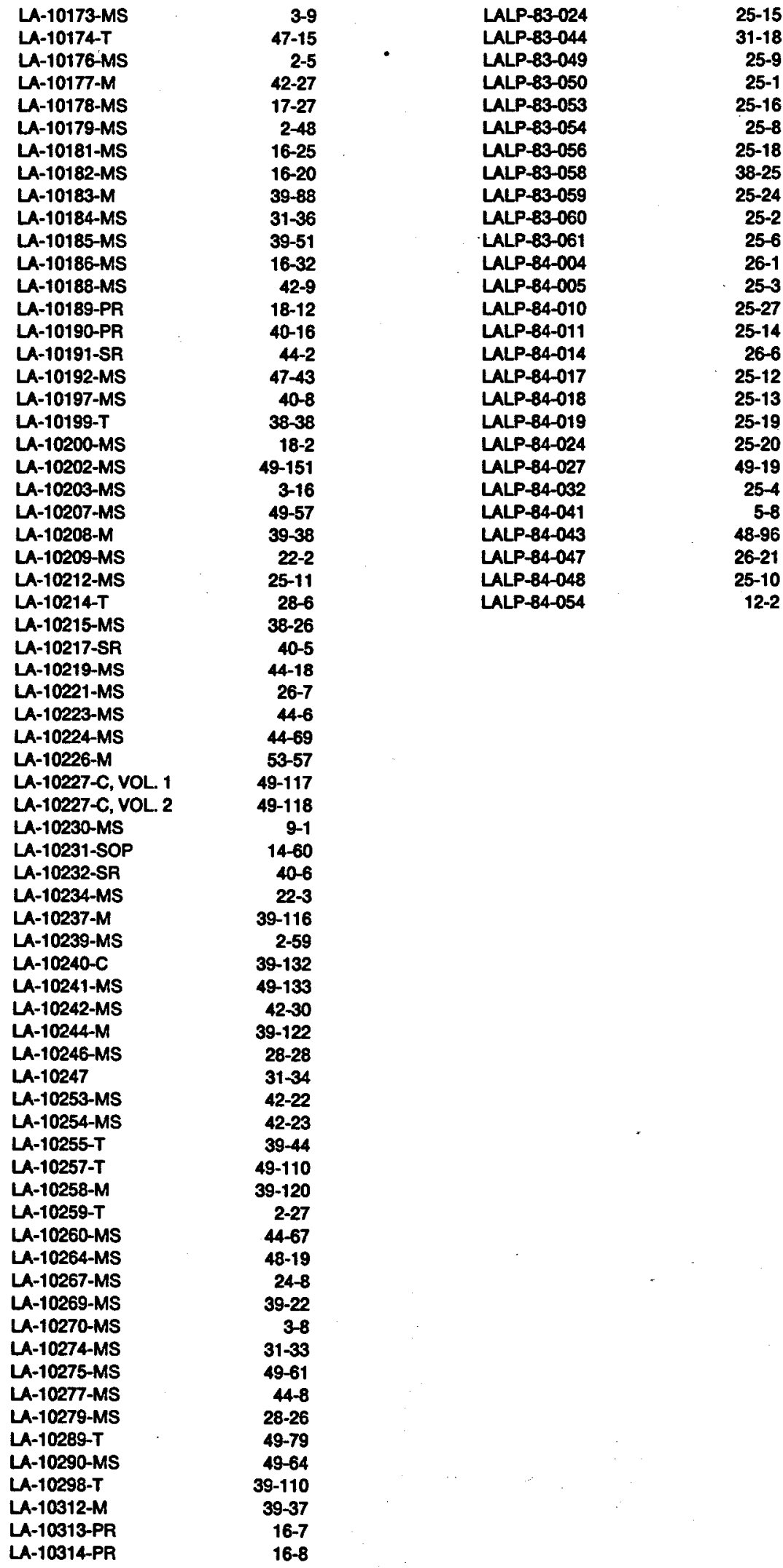

U.S.PATENT-4,421,982 31-1 U.S.PATENT-4,424,491 31-3 U.S.PATENT-4,424,858 $31-2$ U.S.PATENT-4,424,866 14-44 U.S.PATENT-4,426,620 14-2 U.S.PATENT-4,429,228 14-29 U.S.PATENT-4,430,451 38-22 U.S.PATENT-4,437,064 14-4 U.S.PATENT-4,437,456 14-28 U.S.PATENT-4,437,958 49-22 U.S.PATENT-4,438,394 14-8 U.S.PATENT-4,439,860 14-51 U.S.PATENT $-4,441,024 \quad 14-66$ U.S.PATENT-4,442,522 49-9 U.S.PATENT-4,442,714 14-47 U.S.PATENT-4,443,072 14-56 U.S.PATENT-4,445,037 28-2 U.S.PATENT-4,447,151 37-6 U.S.PATENT-4,447.153 49-3 U.S.PATENT-4,447,303 32-3 U.S.PATENT-4,448,622 14-11 U.S.PATENT-4,459,153 5-52 U.S.PATENT-4,459,268 5-44 U.S.PATENT-4,459,811 14-40 U.S.PATENT-4,464,330 14-5 U.S.PATENT-4,464,413 14-43 U.S.PATENT-4,464,416 49-83 U.S.PATENT-4,464,616 14-61 U.S.PATENT-4,468,776 14-58 U.S.PATENT-4,471,697 14-7 U.S.PATENT-4,473,875 14-32 U.S.PATENT-4,479,059 28-17 U.S.PATENT-4,481,048 5-25 U.S.PATENT-4,481,371 5-42 U.S.PATENT $-4,481,999 \quad 14-46$ U.S.PATENT-4,483,816 14-3 U.S.PATENT-4,487,738 $5-43$ U.S.PATENT-4,490,709 14-31 


\section{PUBLICATION TABULATION}

\section{A. JOURNAL ARTICLES - 587 IN 161 JOURNALS}

ACCOUNTS OF CHEMICAL RESEARCH - 1 ACTA CRYSTALLOGRAPHICA - 1 ADVANCES IN CRYOGENIC ENGINEERING - 4 ADVANCES IN CYCLIC NUCLEOTIDE AND PROTEIN POSPHORYLATION RESEARCH - 2 ADVANCES IN LASER SPECTROSCOPY - 1 ADVANCES IN SPACE RESEARCH - 1 AGRICULTURAL METEOROLOGY - 1 AMERICAN INDUSTRIAL HYGIENE ASSOCIATION. JOURNAL - 1 ANALYTICAL CHEMISTRY - 3

ANNALS OF PHYSICS - 2

ANNALS OF THE NEW YORK ACADEMY OF SCIENCES - 1

APPLICATIONS OF SURFACE SCIENCE - 1

APPUIED OPTICS - 4

APPLIED PHYSICS LETTERS - 10

APPLIED SPECTROSCOPY - 2

ASTRONOMICAL JOURNAL - 2

ASTRONOMY AND ASTROPHYSICS - 1

ASTROPHYSICAL JOURNAL - 21

ASTROPHYSICAL JOURNAL. SUPPLEMENT SERIES. - 1 ASTROPHYSICS AND SPACE SCIENCE - 1 BIOCHEMISTRY - 1

BIOPHYSICAL JOURNAL - 4

BULLETIN OF THE AMERICAN ASTRONOMICAL SOCIETY - 1

BULLETIN OF THE ATOMIC SCIENTISTS - 1

CANADIAN JOURNAL OF CHEMISTRY - 1

CHEMICAL ENGINEERING COMMUNICATIONS - 1

CHEMICAL PHYSICS LETTERS - 8

CLAYS AND CLAY MINERALS - 1

COMBUSTION SCIENCE TECHNOLOGY - 1

COMMENTS ON NUCLEAR PARTICLES AND PHYSICS - 2

COMMENTS ON PLASMA PHYSICS AND CONTROLLED FUSION - 3

COMPUTER PHYSICS COMMUNICATIONS - 2

CONTEMPORARY MATHEMATICS - 1

CYTOGENETICS AND CELL GENETICS - 1

CYTOMETRY - 2

ENERGY AND BUILDINGS - 1

ENVIRONMENTAL SCIENCE AND TECHNOLOGY - 1

EOS - 3

EXPERIMENTAL CELL RESEARCH - 1

EXPERIMENTS IN FLUIDS - 1

EXPLORERS JOURNAL- 1

FERROELECTRICS - 1

FUSION TECHNOLOGY - 1

GEOCHIMICA ET COSMOCHIMICA ACTA - 1

GEOPHYSICAL RESEARCH LETTERS - 10

HEALTH PHYSICS - 4

HYPERFINE INTERATIONS - 3

IEEE TRANSACTIONS ON NUCLEAR SCIENCE - 8

IMMUNOLOGICAL COMMUNICATIONS - 1

INORGANIC CHEMISTRY - 2

INTERNATIONAL JOURNAL OF ENGINEERING SCIENCE - 3

INTERNATIONAL JOURNAL OF MULTIPHASE FLOW - 1

INTERNATIONAL JOURNAL OF QUANTUM CHEMISTRY. SYMPOSIUM - 1

JOURNAL DE PHYSIQUE. COLLOQUE - 1

JOURNAL OF APPLIED PHYSICS - 17

JOURNAL OF ATMOSPHERIC AND TERRESTRIAL PHYSICS - 1

JOURNAL OF BIOLOGY AND CHEMISTRY - 3

JOURNAL OF BIOMOLECULAR STRUCTURE \& DYNAMICS - 1

JOURNAL OF CATALYSIS - 1

JOURNAL OF CELLULAR PHYSIOLOGY - 1

JOURNAL OF CHEMICAL AND ENGINEERING DATA - 2

JOURNAL OF CHEMICAL PHYSICS - 32

JOURNAL OF CHROMATOGRAPHIC SCIENCE - 1

JOURNAL OF CHROMATOGRAPHY - 1
JOURNAL OF CLIMATE AND APPLIED METEOROLOGY - 2 JOURNAL OF COLLOID AND INTERFACE SCIENCE - 1 JOURNAL OF COMPUTATION AND APPLIED MATHEMATICS - 1 JOURNAL OF COMPUTATIONAL PHYSICS - 6 JOURNAL OF ENERGY AND MATTER - 2 JOURNAL OF ENVIRONMENTAL QUALITY - 1 JOURNAL OF FLUID MECHANICS - 2 JOURNAL OF GEOPHYSICAL RESEARCH - 25 JOURNAL OF HETEROCYCLIC CHEMISTRY - 1 JOURNAL OF IMMUNOLOGICAL METHODS - 1 JOURNAL OF IMMUNOLOGY - 2 JOURNAL OF LABELLED COMPOUNDS ANO RADIO-

PHARMACEUTICALS - 3

JOURNAL OF LOW TEMPERATURE PHYSICS - 3

JOURNAL OF MAGNETISM AND MAGNETIC MATERIALS - 1 JOURNAL OF MATERIALS FOR ENERGY SYSTEMS - 1 JOURNAL OF MATHEMATICAL PHYSICS - 5 JOURNAL OF MOLECULAR SPECTROSCOPY - 2 JOURNAL OF NON-CRYSTALLINE SOLIDS - 1 JOURNAL OF NUCLEAR MATERIALS - 5 JOURNAL OF OCCUPATIONAL MEDICINE - 1 JOURNAL OF PHYSICAL CHEMISTRY - 8 JOURNAL OF PHYSICS. A. MATHEMATICAL AND GENERAL - 3 JOURNAL OF PHYSICS. 8. ATOMIC AND MOLECULAR PHYSICS - 2 JOURNAL OF PHYSICS. C. SOLID STATE PHYSICS - 1 JOURNAL OF OUANTITATIVE SPECTROSCOPY AND RADIATIVE TRANSFER - 1

JOURNAL OF RADIOANALYTICAL AND NUCLEAR CHĖMISTRY - 1 JOURNAL OF STATISTICAL PHYSICS - 2

JOURNAL OF THE AIR POLLUTION CONTROL ASSOCIATION - 2

JOURNAL OF THE AMERICAN CERAMIC SOCIETY - 4

JOURNAL OF THE AMERICAN CHEMICAL SOCIETY - 4

JOURNAL OF THE AMERICAN STATISTICAL ASSOCIATION - 1

JOURNAL OF THE ATMOSPHERIC SCIENCES - 1

JOURNAL OF THE CHEMICAL SOCIETY. CHEMICAL

COMMUNICATIONS - 2

JOURNAL OF THE ELECTROCHEMICAL SOCIETY - 1

JOURNAL OF THE OPTICAL SOCIETY OF AMERICA - 4

JOURNAL OF THE WATER POLLUTION CONTROL FEDERATION - 1

JOURNAL OF VACUUM SCIENCE AND TECHNOLOGY - 5

JOURNAL OF WILDLIFE MANAGEMENT - 2

LOG ANALYST - 1

LOS ALAMOS SCIENCE - 20

MACROMOLECULES - 1

MATHEMATICAL BIOSCIENCES - 1

METALLURGICAL TRANSACTIONS - 1

MOLECULAR AND CELLULAR BIOLOGY - 1

MONTHLY NOTICES OF THE ROYAL ASTRONOMICAL SOCIETY - 1

NATURE - 2

NUCLEAR FUSION - 2

NUCLEAR INSTRUMENTS AND METHODS - 12

NUCLEAR INSTRUMENTS AND METHODS IN PHYSICS RESEARCH - 5

NUCLEAR PHYSICS - 6

NUCLEAR SCIENCE AND ENGINEERING - 3

NUCLEAR TECHNOLOGY/FUSION - 1

NUCLEAR TECHNOLOGY - 1

NUCLEIC ACIDS RESEARCH - 2

OPTICAL ENGINEERING - 1

OPTICS LETTERS - 3

ORGANOMETALLICS - 1

PARALLEL COMPUTING - 1

PARTICLE ACCELERATORS - 1

PHOTOCHEMISTRY AND PHOTOBIOLOGY - 1

PHYSICA - 3

PHYSICAL REVIEW - 87

PHYSICAL REVIEW LETTERS - 27

PHYSICS LETTERS - 19

PHYSICS OF FLUIDS - 22 
PHYSICS TODAY - 2

PLANT PHYSIOLOGY - 1

PLATING AND SURFACE FINISHING - 1

PROCEEDINGS OF THE NATIONAL ACADEMY OF SCIENCES - 1

PROGRESS IN NUCLEAR ENERGY - 1

PROGRESS IN PARTICLE AND NUCLEAR PHYSICS - 1

PROPELLANTS, EXPLOSIVES, PYROTECHNICS- 1

PUBLICATIONS OF THE ASTRONOMICAL SOCIETY OF THE PACIFIC - 1

PURE AND APPLIED CHEMISTRY - 1

RADIATION PROTECTION DOSIMETRY - 1

RADIATION RESEARCH - 3

REVIEW OF SCIENTIFIC INSTRUMENTS - 10

REVIEWS OF MODERN PHYSICS - 1

RISK ANALYSIS - 1

SCIENCE - 1

SCIENCE OF TSUNAMI HAZARDS - 1

SCRIPTA METALLURGICA - 1

SIAM JOURNAL ON NUMERICAL ANALYSIS - 1

SKY AND TELESCOPE - 1

SOLAR PHYSICS - 1

SOLID STATE COMMUNICATIONS - 1

SOUID STATE PHYSICS - 1

SPECTROCHIMICA ACTA - 3
SURFACE SCIENCE - 2

SYNTHETIC METALS - 1

TERRA COGNITA - 1

TOXICOLOGY - 1

TRANSPORT THEORY AND STATISTICAL PHYSICS - 3

WATER, AIR AND SOIL POLLUTION - 1

WAVE MOTION - 1

WELDING JOURNAL - 1

WILDLIFE SOCIETY BULLETIN - 1

B. BOOKS - 2

C. CHAPTERS OF BOOKS AND ENCYCLOPEDIA ARTICLES - 24

D. PAPERS PUBLISHED IN CONFERENCE PROCEEDINGS - 192

E. ABSTRACTS OF PAPERS - 56

F. PATENTS - 38

G. LOS ALAMOS NATIONAL LABORATORY REPORTS - 287

H. PAPERS ISSUED AS LOS ALAMOS REPORTS BY TIC AND NTIS - 533 